8unytoous uvag-ısv 7 fo spoaff aut 8un apow

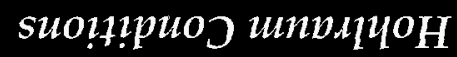
fo uoupvzuajovivyว jo7uaumadx

s8umvis noupvufta

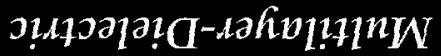
ศวนวเงนศन- $น 8 ! H$

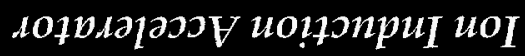

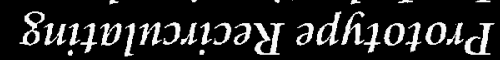
v pinmol ssarsord

sasnos fivy-X fo uoutvzumuto

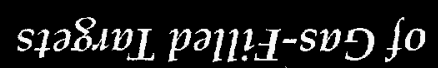
8u?7sal puv uọ7vouqvi
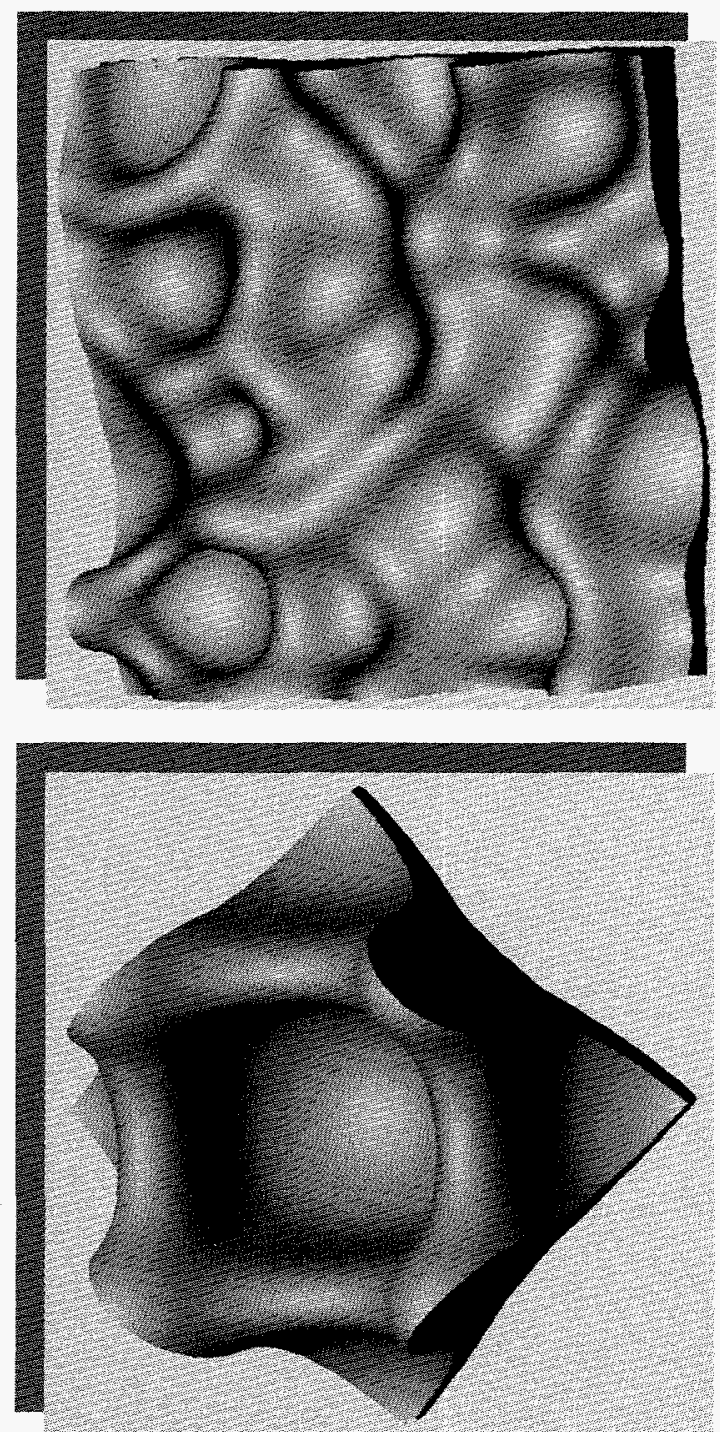

E saqunN 'G aunๆo $\Lambda$ G66I aun -1 l. $d$ d

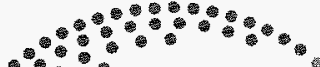

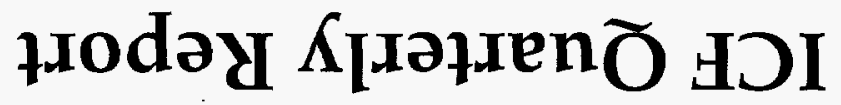

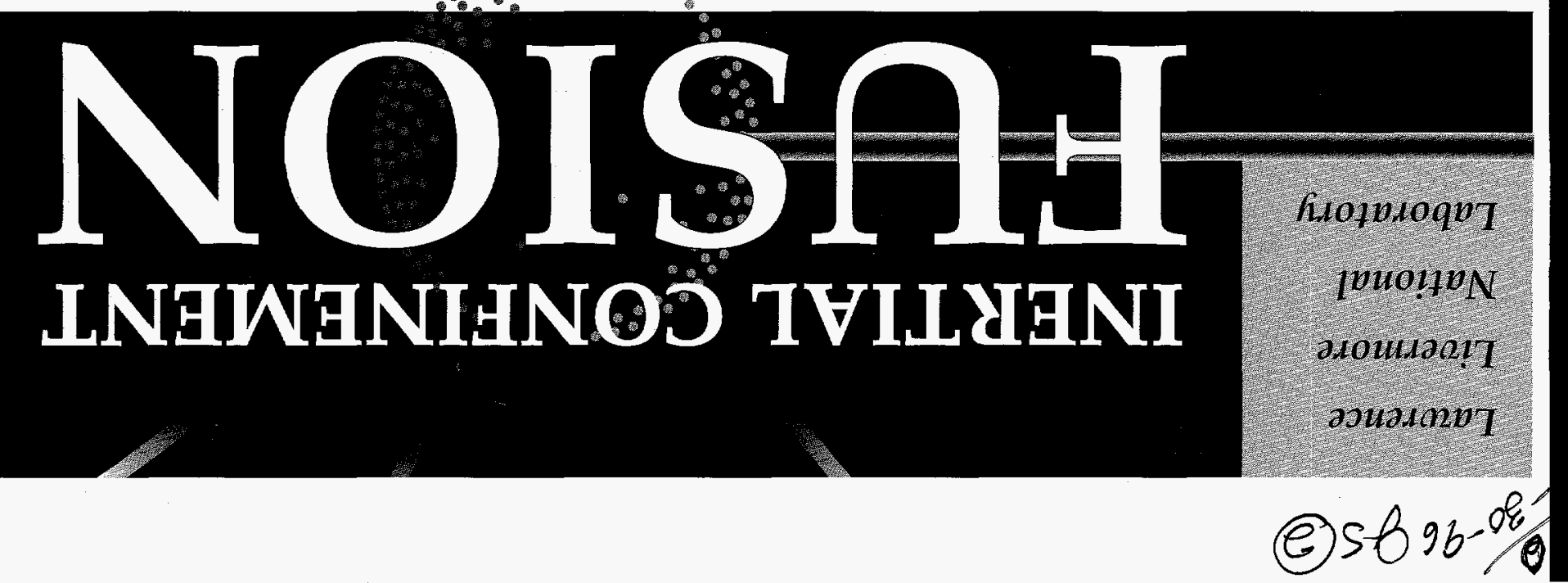


The ICF Quarterly Report is published four times each fiscal year by the Inertial Confinement Fusion Program at the Lawrence Livermore National Laboratory. The journal reports selected current research within the ICF Program. Major areas of investigation presented here include fusion target theory and design, target fabrication, target experiments, and laser and optical science and technology. Questions and comments relating to the technical content of the journal should be addressed to the ICF Program Office, Lawrence Livermore National Laboratory, P.O. Box 5508, Livermore, CA 94551.

The Cover: These three-dimensional (3-D) isodensity contour plots were obtained from simulations of ablative hydrodynamic instabilities in foils performed with the HYDRA radiation hydrodynamics code. The images on the top show broad bubbles surrounded by narrow spike sheets during the nonlinear stage of instability evolution. They are seen from the side of the foil ablated by $x$ rays. The foil on the left was initialized with a cartesian 3-D mode surface perturbation while the foil on the right had the two-dimensional mode with the same corresponding wave number. From a similar perspective, the bottom-left image shows the nonlinear development of bubbles and spike sheets for a foil with a random multimode perturbation. Taken later in time, the bottom-right image of the back side of the foil shows the largest bubbles, which broaden and rise most rapidly. For details, see the article "ThreeDimensional Simulations of Ablative Hydrodynamic Instabilities in Indirectly Driven Targets," p. 168.

UCRL-LR-105821-95-3

Distribution Category UC-712 April-June 1995

Printed in the United States of America Available from

National Technical Information Service

U.S. Department of Commerce 5285 Port Royal Road

Springfield, Virginia 22161

Price codes: printed copy A03, microfiche A01.
This document was prepared as an account of work sponsored by an agency of the United States Government. Neither the United States Government nor the University of California nor any of their employees makes any warranty, express or implied, or assumes any legal liability or responsibility for the accuracy, completeness, or usefulness of any information, apparatus, product, or process disclosed, or represents that its use would not infringe privately owned rights.

Reference herein to any specific commercial products, process, or service by trade name, trademark, manufacturer, or otherwise, does not necessarily constitute or imply its endorsement, recommendation, or favoring by the United States Government or the University of California. The views and opinions of authors expressed herein do not necessarily state or reflect those of the United States Government or the University of California and shall not be used for advertising or product endorsement purposes.

Work performed under the auspices of the U.S. Department of Energy by Lawrence Livermore National Laboratory under Contract W-7405-Eng-48. 


\section{DISCLAMMER}

Portions of this document may be illegible in electronic image products. Images are produced from the best available original document. 


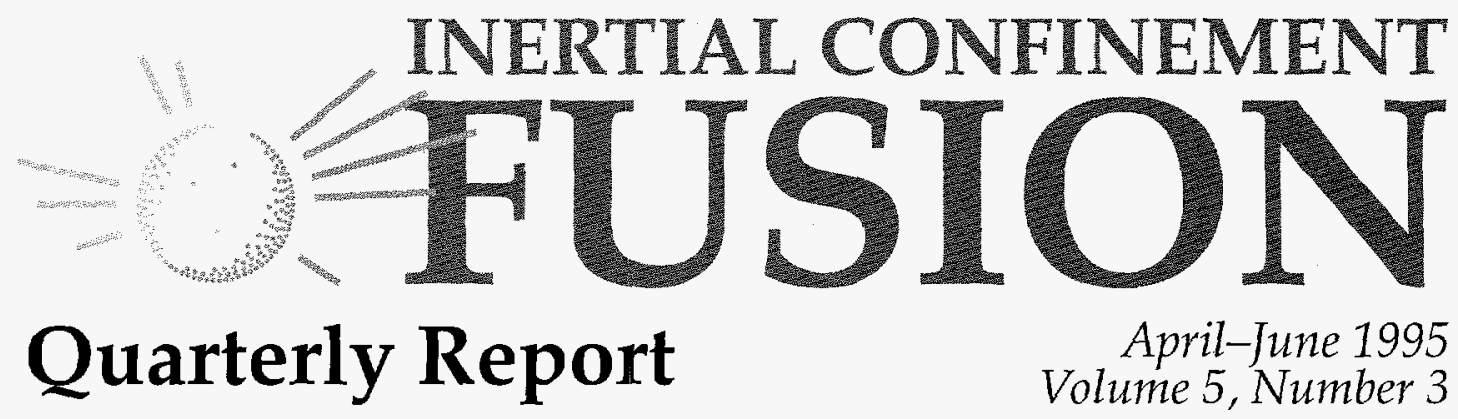

\section{In this issue:}

Foreword

Fabrication and Testing of Gas-Filled Targets

for Large-Scale Plasma Experiments on Nova

This article describes the target materials and fabrication processes employed to produce gas-filled targets for Nova. Fabrication techniques for both open- and closed-geometry targets are discussed. The testing methods used for the hardware and materials used in these gas-tight targets are also included.

\section{Optimization of X-Ray Sources from a High-Average-Power} Nd:Glass Laser-Produced Plasma for Proximity Lithography A laser-produced plasma is a strong candidate for an $x$-ray source for proximity lithography. Using various solid targets, we have measured the x-ray yields and spectra from plasmas generated with a prototype high-average-power pulsed Nd:glass laser.

\section{Three-Dimensional Simulations of Ablative Hydrodynamic} Instabilities in Indirectly Driven Targets

This article examines the first simulations and experiments to study the effect of three-dimensional (3-D) perturbation shape on instability growth and saturation in indirectly driven targets. Simulations are performed using a new 3-D radiation hydrodynamics code we developed called HYDRA.

Progress Toward a Prototype Recirculating Ion Induction Accelerator The U.S. Heavy Ion Fusion program is developing the physics and technology of ion induction accelerators as drivers for Inertial Fusion Energy and post-National Ignition Facility ICF research. This article describes a newly initiated experimental effort aimed at developing a low-cost approach to highcurrent ion acceleration based on recirculation of the ion beams. The goal of this experimental sequence is an operating prototype recirculator.

\section{High-Efficiency Multilayer-Dielectric Diffraction Gratings}

Multilayer-dielectric diffraction gratings compared with conventional metallic gratings offer increased efficiency and laser damage threshold when used for pulse compression in ultrashort pulse, highintensity laser systems. This article describes theoretical and experimental results concerning the

Scientific Editor

S. Gail clendinning

Editorial Staff Tason Carpenter. Al T. Miguel Peter W Murphy Dablie P Schleich

Design Staff

Daniels. Moore

Att Staff

Linda L. Wiseman

\section{Experimental Characterization of Hohlraum Conditions by X-Ray Spectroscopy}

$X$-ray spectroscopy can be a powerful method for diagnosing the plasma conditions in laser-produced plasmas. In ICF applications, the closed geometry of the hohlraum is a unique environment because of its intense radiation field. This article discusses three types of experiments that demonstrate the breadth of measurements that can be performed using spectroscopic techniques.

\section{Modeling the Effects of Laser-Beam Smoothing on Filamentation and Stimulated Brillouin Backscattering}

Using a newly developed three-dimensional code (F3D), our results show that filamentation can be controlled and stimulated Brillouin backscattering can be reduced by using random phase plates and small $f$-numbers or smoothing by spectral dispersion with high bandwidth.

\section{Facilities Report}

Publications

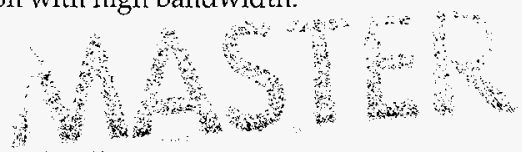

F-1

P.1 20 



\section{FOREWORD}

This issue of the ICF Quarterly has seven articles covering developments in target fabrication, target design, Nova target experiments, $x$-ray lithography, laser science, and reactor design.

During FY 1995, the ICF program has devoted considerable effort to the understanding of basic plasma instabilities, such as stimulated Raman and Brillouin scattering (SRS and SBS). These instabilities are in the context of laser interactions with the plasma in a hohlraum, particularly for the spatial scales and plasma conditions expected in an ignition-scale hohlraum. Two of the articles discuss this subject. In the first article, "Fabrication and Testing of Gas-Filled Targets for Large-Scale Plasma Experiments on Nova," the authors describe the steps in the process of fabricating and testing two kinds of gas targets for plasma instability experimentsgas-bag-style targets that have a fairly unlimited or open diagnostic view of the plasma, and hohlraum-based targets that have a more restricted diagnostic view of the plasma. The second article, "Modeling the Effects of Laser-Beam Smoothing on Filamentation and Stimulated Brillouin Backscattering," discusses beam smoothing by temporal incoherence and its effect on reflectivity due to stimulated Brillouin backscattering. The addition of bandwidth (temporal incoherence) is found to be an effective tool for reducing reflectivity compared with static beams, and the expected reduction in reflectivity with $f$-numbers does not occur in these simulations.

Electron temperature $T_{\mathrm{e}}$ of the plasma is one important parameter for modeling and understanding plasma instability experiments. In "Experimental Characterization of Hohlraum Conditions by X-Ray Spectroscopy," the authors report on measurements of $T_{\mathrm{e}}$ by inserting tracer foils in a hohlraum and examining the detailed spectral emission from the foil as a function of time. A similar technique may also be used to measure ablation rates and to characterize drive in lined hohlraums by placing the tracer on a hohlraum wall.

Understanding hydrodynamic instabilities is crucial to designing imploding capsules. Until recently, most simulations have been unable to examine the three-dimensional (3-D) character of an accelerating foil or an imploding capsule. In "Three-Dimensional Simulations of Ablative Hydrodynamic Instabilities in Indirectly Driven Targets," the authors present results from a new arbitrary Lagrangian-Eulerian code, which simulate indirectly driven foil experiments and predict an ongoing series of capsule experiments. The 3-D effects indicate that certain initial surface shapes are the most destructive for imploding capsules.

High-intensity (petawatt) lasers are essential to the approach to capsule ignition, known as the fast-ignitor scheme; and high-damage threshold dielectric gratings are essential to petawatt lasers. In "High-Efficiency Multilayer-Dielectric Diffraction Gratings," the authors describe design considerations and techniques used successfully to manufacture dielectric gratings. The dielectric is an oxide-based multilayer structure, and the gratings have a damage threshold nearly 10 times that for metallic gratings and efficiencies of better than $96 \%$.

Our investigation into industrial applications of laser-based $x$-ray sources and $x$-ray optics is represented in "Optimization of X-Ray Sources from a High-Average-Power Nd:Glass LaserProduced Plasma for Proximity Lithography." The authors describe absolute measurements of $x$-ray production from laser-irradiated samples in an energy region of interest to Si microcircuit manufacture. They find that a long-pulse (12-ns) laser run at a high average power $(120 \mathrm{~W}) \mathrm{can}$ produce $x$ rays at efficiencies of $10-15 \%(2 \pi \mathrm{sr}$ ).

The article "Progress Toward a Prototype Recirculating Ion Induction Accelerator" discusses design and experiments in the field of heavy ion beam-driven inertial fusion. Specifically, a small prototype recirculator has been designed to produce $320 \mathrm{keV} \mathrm{K}{ }^{+}$ions by accelerating the beam in a circle over 15 laps. The authors report on experimental results and modeling of various beam parameters in the matching and linear transport sections of such an accelerator.

S. Gail Glendinning

Scientific Editor 


\title{
FAbrication And Testing of GAs-Filled TARgets For LARge-Scale Plasma Experiments on Nova
}

\author{
G. F. Stone M.R. Spragge \\ C. J. Rivers* R. J. Wallace
}

\section{Introduction}

The proposed next-generation ICF facility, the National Ignition Facility (NIF), is designed to produce energy gain from $x$-ray heated "indirect-drive" fuel capsules. For indirect-drive targets, laser light heats the inside of the Au hohlraum wall and produces $x$ rays which in turn heat and implode the capsule to produce fusion conditions in the fuel. ${ }^{1}$ Unlike Nova targets, in NIF-scale targets laser light will propagate through several millimeters of gas, producing a plasma, before impinging upon the Au hohlraum wall. The purpose of the gas-produced plasma is to provide sufficient pressure to keep the radiating Au surface from expanding excessively into the hohlraum cavity. Excessive expansion of the Au wall interacts with the laser pulse and degrades the drive symmetry of the capsule implosion.

We have begun an experimental campaign on the Nova laser to study the effect of hohlraum gas on both laser-plasma interaction and implosion symmetry. ${ }^{1,2}$ In our current NIF target design, the calculated plasma electron temperature is $T_{\mathrm{e}} \approx 3 \mathrm{keV}$ and the electron density is $n_{\mathrm{e}} \approx 10^{21} \mathrm{~cm}^{-3}$. To simulate NIF conditions in a Nova target requires a target with a gas confined. in an $\sim 0.01 \mathrm{~cm}^{3}$ vol. at $\approx 1 \mathrm{~atm} .^{2}$ These gas-filled targets are calculated to produce the required plasma conditions based on an initial gas fill of $1 \mathrm{~atm}$ neopentane $\mathrm{C}_{5} \mathrm{H}_{12}$. To measure the $T_{\mathrm{e}}$ of the plasma by spectroscopic line ratios, $\mathrm{Ar}$ and $\mathrm{Cl}$ bearing gases are added to the mixtures. Metal coated carbon fibers and plastic foils are added as an additional spectroscopic temperature diagnostic. Changes in $n_{\mathrm{e}}$ are made by varying the density of the main and spectroscopic seed gases. To aid in diagnosing the plasma, additional features such as diagnostic shields, x-ray backlighting patches, and imaging slits are added to the target design as experimental requirements dictated.

To study these plasma conditions, targets are being fabricated and shot on the Nova laser using open- and closed-geometric designs. Open geometry refers to the gas-bag style targets that have a fairly unlimited or open diagnostic view of the plasma and are nearly spherical, plastic gas cells built on a metal or plastic support ring. 3,4 Closed geometry refers to indirect-drive style targets with a radiation enclosure that confines the plasma within a cylindrical Au hohlraum. ${ }^{1,2}$ Views of the plasma in a closed-geometry target are through diagnostic holes or slots cut into the wall of the $\mathrm{Au}$ hohlraum and covered with gas-tight patches. This article describes the major steps and processes necessary to fabricate, test, and deliver these gas targets for shots on the Nova laser at LLNL.

\section{Target Design}

To fabricate these gas-filled Nova targets to simulate the NIF-like conditions, we integrate a gas manifold with a pressure transducer, the target and gas fill lines, gas mixing, testing and filling systems, and the Nova target positioner. To ensure a proper fit and interference with existing target handling hardware, we analyze scaled computer-aided design (CAD) drawings of the target and associated components.

Figure 1 is a CAD drawing of a closed-geometry target. CAD drawings are essential for the closedgeometry targets to allow the precise placement of shields, fibers, foils, and other target components relative to diagnostics on the Nova target chamber. In three dimensions, alignment tolerances for the closedgeometry targets are $\pm 25 \mu \mathrm{m}$ and $\leq 2^{\circ}$.

*W. J. Schafer, Livermore, California 


\section{Target Material Selection and Fabrication}

To aid in target and feature alignment at the Nova target chamber, both types of targets need an optically transparent, thin, low- $Z$ ductile material for the gas/ vacuum barrier. The density of the gas is chosen so that when completely ionized and heated to $3 \mathrm{keV}$, the electron pressure will be sufficient to significantly slow the hohlraum wall motion in a NIF target. The density cannot be too large, however, or the plasma $n_{e}$ will exceed the critical value for $0.25 \mu \mathrm{m}$ light propagation, resulting in complete reflection of the incident light. The density of $3 \mathrm{mg} / \mathrm{cm}^{3}$ of neopentane results in a 0.1 critical density $\left(10^{21}\right.$ electrons $\left./ \mathrm{cm}^{3}\right)$, which is calculated to hold off wall motion and to allow light propagation to the wall for $\mathrm{x}$-ray conversion. Ideally, actual NIF hohlraums, or experiments to simulate NIF hohlraum conditions for testing the laser-plasma interaction physics, would have no solid-material gas barriers. Early in a laser shot, these barriers, or windows, are heated to a plasma state and rapidly expand, severely perturbing the interior gas. The windows must be as thin as possible to reduce this perturbation. However, they must be thick enough to withstand the 1-atm pressure difference. In Table 1, we compare several window materials. The thickness required is calculated from the simple hoop- stress formula, $\sigma=\operatorname{Pr} / 2 t$, using appropriate yield stress $\sigma$ for each material. $P$ is the pressure, $r$ the radius of curvature of the window, which is assumed to be $1 \mathrm{~mm}$, and $t$ is its thickness. The equivalent gas thickness is calculated as the distance the initial window will expand to reach $10^{21}$ electrons $/ \mathrm{cm}^{3}$, based on the initial $n_{\mathrm{e}}$ shown in the second column of Table 1 . This equivalent distance must be minimized. Based on these considerations, we chose polyimides as the window material, of which Kapton is a well-known commercial example. Polyimide is a family of plastic $\mathrm{CH}$ polymers used in integrated circuit fabrication. Although the chemical formula for the specific material used is proprietary, it is nominally $\mathrm{C}_{22} \mathrm{H}_{10} \mathrm{~N}_{2} \mathrm{O}_{4}$ with a density of $1.4-1.5 \mathrm{~g} / \mathrm{cm}^{3}$.

The open-geometry targets need a nearly spherical volume of gas and must hold the pressure for 1-3 hr.

TABLE 1. Comparison of window materials.

\begin{tabular}{|c|c|c|c|}
\hline Material & $\begin{array}{l}\text { Electron density } \\
\left(\mathrm{moles} / \mathrm{cm}^{3}\right)\end{array}$ & $\begin{array}{l}\text { Thickness } \\
\text { required }\end{array}$ & $\begin{array}{l}\text { Equivalent gas } \\
\text { thickness ( } \mathrm{mm} \text { ) }\end{array}$ \\
\hline $\mathrm{Si}_{3} \mathrm{~N}_{4}$ & 1.72 & $0.2 \mu \mathrm{m}$ & 206 \\
\hline Lexan & 0.74 & $0.8 \mu \mathrm{m}$ & 355 \\
\hline Kapton & 0.72 & $0.3 \mu \mathrm{m}$ & 130 \\
\hline Parylene & 0.56 & $0.8 \mu \mathrm{m}$ & 268 \\
\hline
\end{tabular}

East
North

South

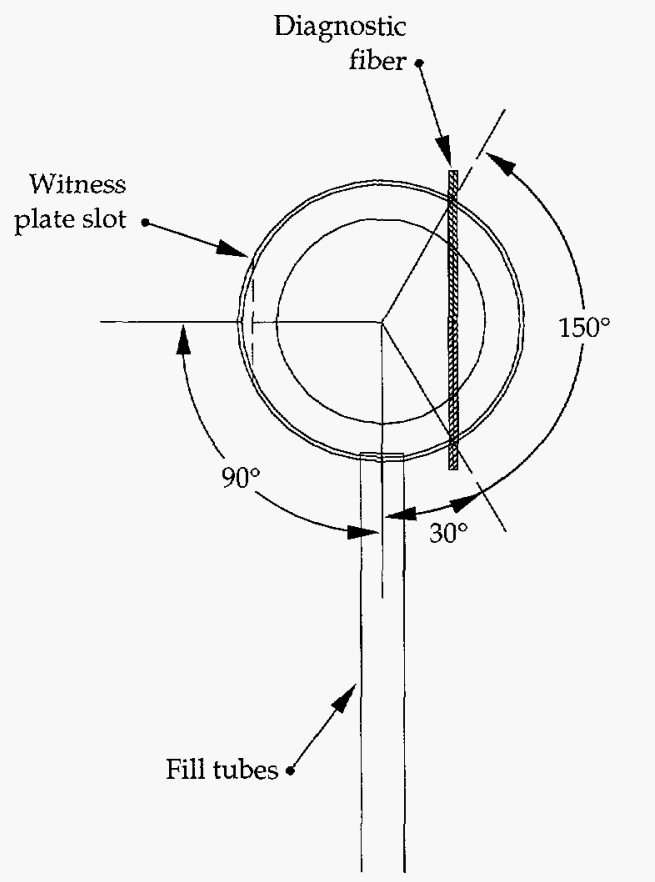

FIGURE 1. A CAD drawing of a closed-geometry target. (10-00-0795-1777pb02) 
The target skin must endure $>1$ atm pressure over an area of $\sim 20 \mathrm{~mm}^{2}$, surrounded by a vacuum of $<10^{-5}$ Torr and be ductile enough to form a nearly spherical bubble from two flat sheets. The gas-bag targets are fabricated using brass, Lucite, and Al support washers $(\sim 400 \mu \mathrm{m}$ wide). Fill tube holes $(270 \mu \mathrm{m}$ diam) are drilled through the side walls of the washers, which are microbead blasted to increase the surface area and to improve the polyimide/epoxy adhesion. A thin sheet of polyimide $(\sim 0.4 \mu \mathrm{m})$ is glued across both sides of a thin washer and two small-diameter stainless-steel fill tubes $(\sim 250 \mu \mathrm{m})$ are passed through the side wall into the inner diameter of the washer and glued in place. Pressurizing the fill lines ( $>20$ psia) causes the films to distend, forming a nearly spherical bubble. Figure 2 is a photograph of a gas-bag target (with a 2.75-mm washer and a $2.4-2.5-\mathrm{mm}$-wide bag).

Nova's Au-hohlraum design serves as the model for the closed-geometry targets. Holes or slots are milled in the side of the target mandrel to view the target's interior (Fig. 3). Fill tube holes are also drilled into the side of the hohlraum mandrel to allow gas filling. After the Au-plated $\mathrm{Cu}$ mandrels are machined and inspected for accuracy, the outer surface is microbead blasted to produce a roughened finish. The mandrels are then leached in a nitric-acid solution $\left(\mathrm{HNO}_{3} \approx 0.5 \mathrm{~N}\right.$ solution) at $60^{\circ} \mathrm{C}$ and etched (1-3 hr). The laser entrance holes (LEHs) and any diagnostic windows in the hohlraum are then covered with a thin $(0.2-0.7-\mu \mathrm{m})$ sheet of polyimide.

The requirement to hold gas behind thin $\mathrm{CH}$ windows complicates target fabrication. The same basic

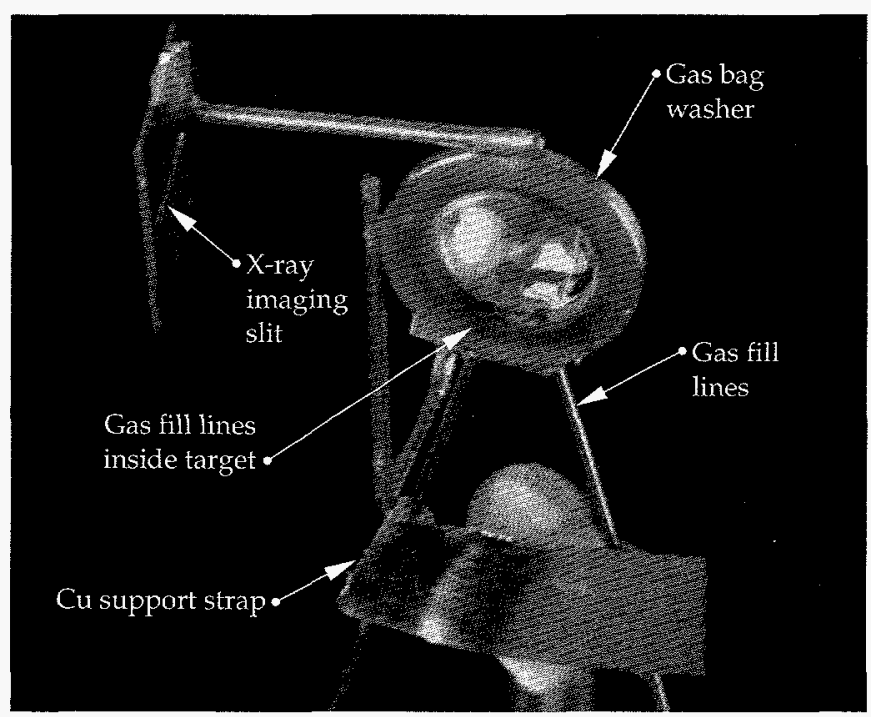

FIGURE 2. Photograph of a gas-bag target with an $x$-ray imaging slit. (10-00-0795-1778pb01) assembly procedures used for non-gas targets are employed on the gas-cell target series. Target fabrication is generally performed using an optical microscope, with micromanipulators holding target parts in place with vacuum chucks and fast curing or UV/visible cured cements to affix target components.

For the open-geometry targets, the final assembly consists of mounting the target on a Nova magnetic target base at the desired orientation. Shields and imaging slits are attached to some of the open-geometry targets. The closed-geometry targets have additional alignment restrictions placed on them by alignment fibers and flags, backlighter patches, diagnostic holes, and slots. The target components are required to be placed within $\pm 25 \mu \mathrm{m}$ and $\leq 2^{\circ}$, relative to the Nova target base.

\section{Gas Specification}

The gas mixture and pressure are specified to mock up the experimental conditions for the point design of the NIF targets, using neopentane $\mathrm{C}_{5} \mathrm{H}_{12}$ as the main gas in this series. To aid in measuring the ion temperature $T_{\text {ion }}$ spectroscopic seed gases $\mathrm{Ar}$ and Freon-13 $\mathrm{CClF}_{3}$ are added in concentrations from 0.25-10 at. \%. The nominal standard target gas for the early experiments was $98 \% \mathrm{C}_{5} \mathrm{H}_{12}+1 \% \mathrm{Ar}+1 \%$ $\mathrm{CClF}_{3}$ at $\sim 1$-atm pressure. To achieve lower target densities, $\mathrm{CO}_{2}, \mathrm{C}_{3} \mathrm{H}_{8}$, and $\mathrm{CH}_{4}$ are used as the base gases. J. Colvin of Los Alamos National Laboratory (LANL) suggested obtaining a deuterated gas $\mathrm{C}_{5} \mathrm{D}_{12}$ to potentially use the fusion neutron yield from the $D$ as a diagnostic for the experiment.

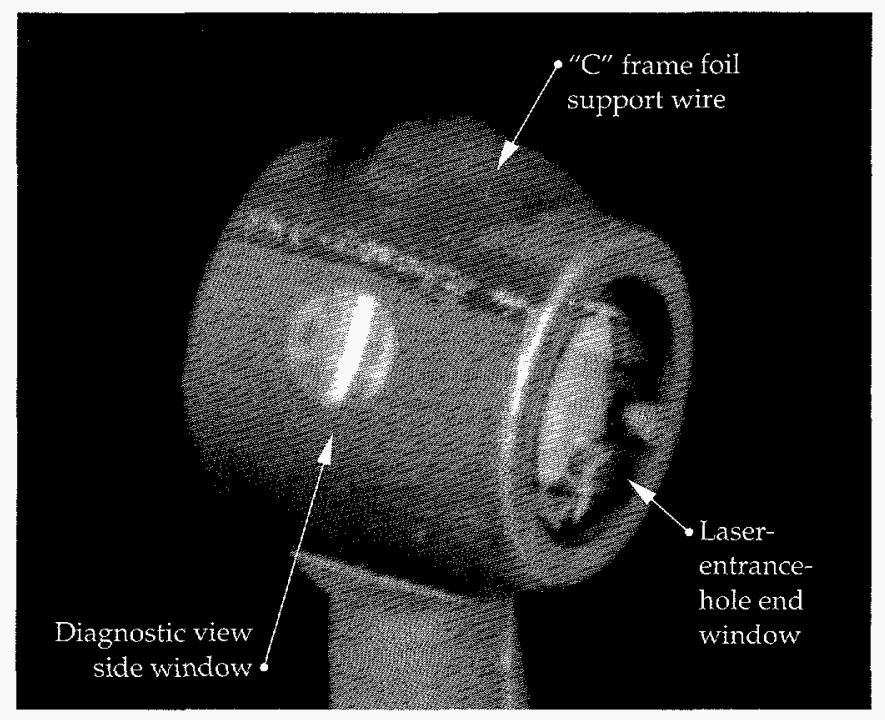

FIGURE 3. Photograph of a closed-geometry target showing the diagnostic view window. (10-00-0795-1780pb01) 
A gas mixing manifold permits mixing of various gases into a sample bottle for later use in filling the targets (Fig. 4). As the main base gas $\mathrm{C}_{5} \mathrm{H}_{12}$ condenses to a liquid at 1300 Torr, an upper limit on the system is set at 1000 Torr. The gas mixture is specified in atomic percentage for the individual components. A diaphragm-style pressure manometer, insensitive to gas densities, monitors pressure in the gas mixing manifold and sample bottle. The sample bottle is connected to the manifold and the system is evacuated. The sample bottle valve is closed and the individual gases are purged three to four times to limit contamination of the final gas mixture. Exceptions to this purging process are $\mathrm{C}_{5} \mathrm{H}_{12}$ and deuterated neopentane $\mathrm{C}_{5} \mathrm{D}_{12}$, due to their limited availability. The $\mathrm{C}_{5} \mathrm{H}_{12}$ and $\mathrm{C}_{5} \mathrm{D}_{12}$ bottles are directly connected to the main manifold with a small prechamber and a valve, which permits a small positive gas pressure to be maintained in the gas regulators and fill lines.

The gas with the lowest percentage in the mixture is first introduced into the manifold, and the pressure is allowed to increase until it reaches the desired partial pressure. The other gases are then added by partial pressure until the total pressure in the sample bottle reaches 1000 Torr.

To interpret the target results, it is necessary to know the ratios of the gases to $<0.1 \mathrm{at} . \%$. The gas mixtures are analyzed using a mass spectrometer with a resolution down to $Z=1(\mathrm{H})$ and a discrimination down to 0.01 at. \%. To improve the accuracy of the analysis, pure samples of each gas are drawn off into small $\left(<75 \mathrm{~cm}^{3}\right)$ sample cylinders to be used as "reference" standards for all the starting gases and when primary gas source bottles are changed.

\section{Target Manifold Design}

A major restriction for the gas target fabrication is the limited space within the Nova target inserter (a cylindrical space 5 in. diam 16 in. long with the center 2 in. occupied by the target pylon); this space limitation drove our hardware choices. Figure 5 is a CAD drawing of a manifold fabricated out of brass to support the transducer, gas flow valves, and target fill lines. The valves were not designed for vacuum sealing but rather for liquid/gas flow metering, and after 6-10 shots, it is necessary to change the valves because they become one of the main sources of leaks on assembled targets.

A strain-gage-style, absolute-pressure transducer monitors manifold gas pressure. Prior to using this type of transducer, we field tested the unit to ensure its ability to operate not only at atmosphere on the bench but also in vacuum in the Nova target chamber. When the target is shot on Nova, a plasma is produced that has a designed $T_{e}$ of $3 \mathrm{keV}$; to electrically protect the transducers, we added a Zener diode to each connector line on the transducer cable to strip off voltage spikes $>50 \mathrm{~V}$. The initial test transducer was calibrated before and after the first laser shot to check for any anomalies. The postshot transducer voltage values were within $\pm 0.001 \mathrm{~V}$ of the preshot values, verifying their robustness.

The early gas-cell targets used a flow-through method to fill the targets with gas, and two valves and gas lines were used on the manifold. One port on the target manifold was connected to the gas sample bottle. The gas flowed until it was estimated that all of the air in the target assembly had been displaced with the sample gas. The current system of evacuated backfill of targets retains the two valves to safeguard against one of the
FIGURE 4. Photograph of a gas mixing system on an optical bread board table. (10-00-0795-1779pb01)

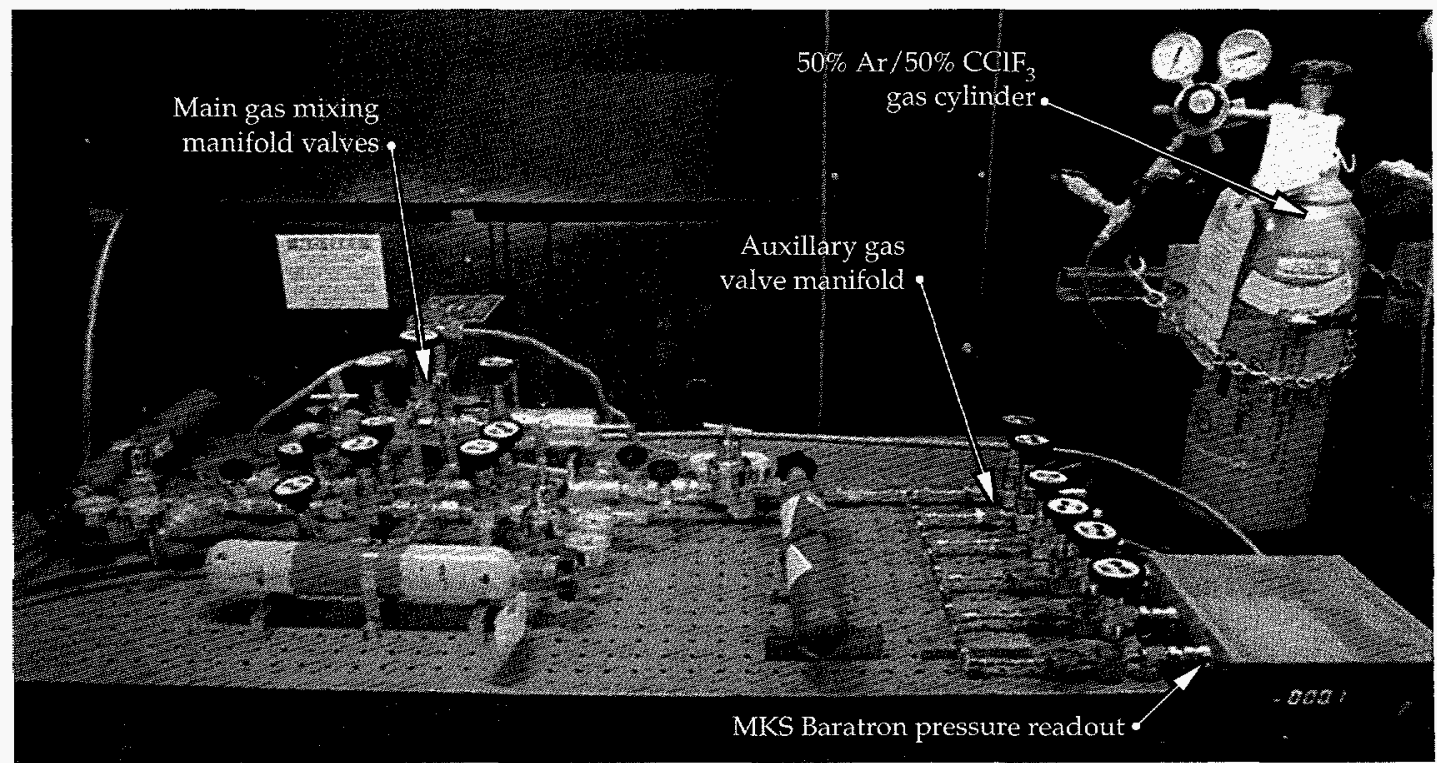


fill lines being blocked. ${ }^{5}$ A pair of 1-mm-o.d. stainlesssteel fill lines is attached to the top of the manifold. Plastic tubing is used to connect the manifold to the target. The target has stainless-steel fill lines $(\sim 250 \mu \mathrm{m}$ o.d.) with smaller i.d. plastic tubing used to inter-connect the target to the plastic tubing on the manifold.

A separate pressure testing system, fabricated by LANL, ${ }^{5}$ is used to test the target manifold. The test procedure is divided into three steps: (1) The target manifold is tested as a standalone unit. (2) The target body is tested and certified gas tight. (3) The assembled target attached to the manifold is tested as an integrated system before filling.

\section{Target Filling Hardware and Procedures}

The first targets were filled by flowing gas through the target, a method that used a large quantity of gas to ensure that only the sample gas remained inside the target. The new method, using an evacuation/backfill chamber, significantly reduces the total gas used per target. ${ }^{5}$

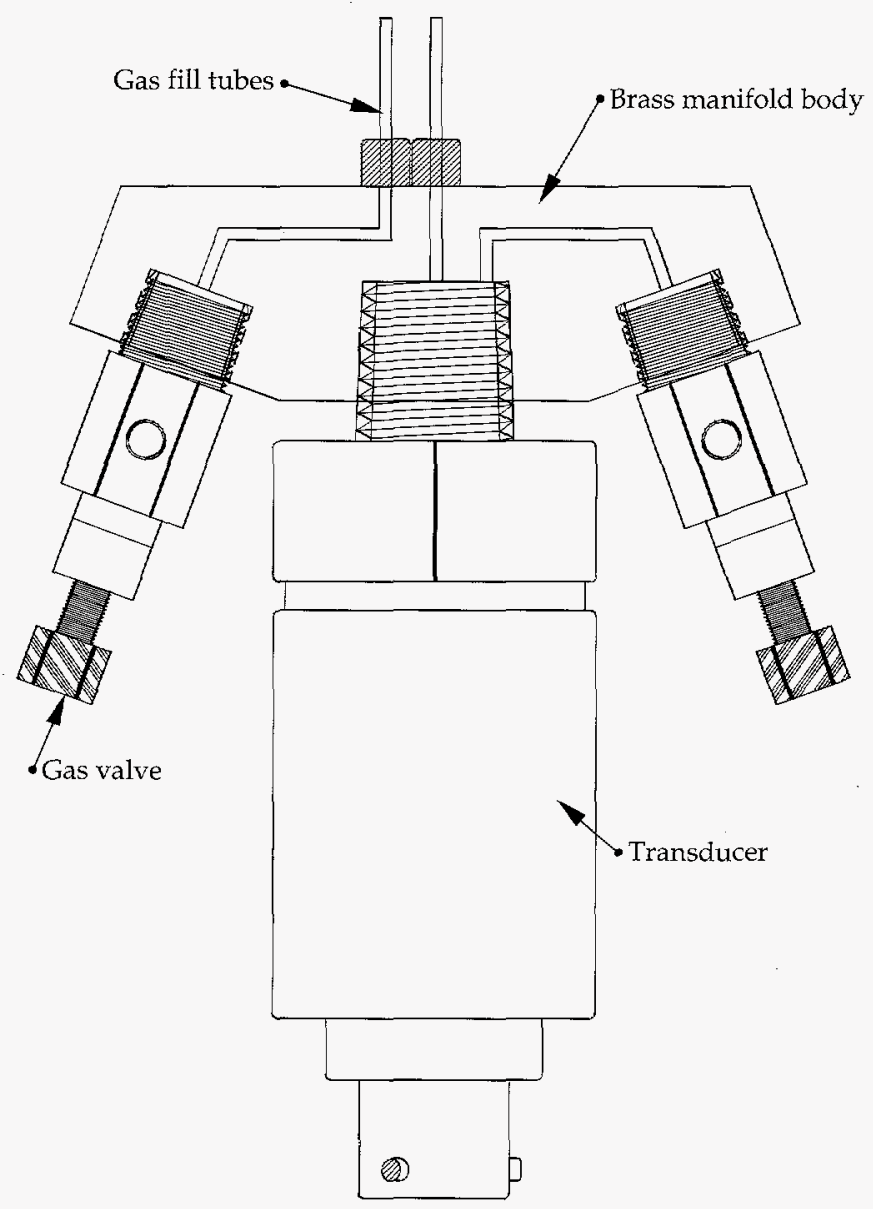

LLNL 10 beam gas manifold

FIGURE 5. A CAD drawing of a target manifold with a transducer, valves, and gas fill lines. (10-00-0795-1781pb01)
The target filling manifold, with its calibrated pressure transducer attached, allows monitoring of internal target pressure at shot time. From this calibrated transducer, a pair of points for pressure vs voltage for vacuum and atmosphere are measured and the resulting values allow the final target pressure at shot time to be calculated. A mechanical manometer is also placed in-line with the fill chamber to monitor the external target pressure in the 1-200-Torr range. A small valving manifold is attached to the evacuation/backfill chamber to control the independent pumping of the target's interior and exterior and filling the target. Figure 6 is a photograph of this gas filling system.

The target and the gas fill line are connected to the target manifold. A purge line ( $\sim 0.25-0.5$ psig) is attached to the target manifold to verify that the fill lines are not blocked. The assembly is then placed in the evacuation/ backfill chamber and the air around the target is slowly pumped out until the chamber pressure reaches $\sim 5 \mathrm{~mm}$ $\mathrm{Hg}$, then the interior of the target is slowly pumped out. Due to the low conductance of the fill tubes (100-250 $\mu \mathrm{m}$ ), the pumping continues for $\sim 15 \mathrm{~min}$ to fully remove the air from the target interior.

Once the base pressure inside the target is reached, the valves to the gas sample bottle are opened and the pressure inside the target is monitored. After the pressure inside the target has increased to the desired value, the chamber is slowly vented to atmosphere. The target manifold valves are closed and the transducer reading is observed for drift-if the target pressure is stable, the fill line is disconnected and the target is ready for delivery to Nova.

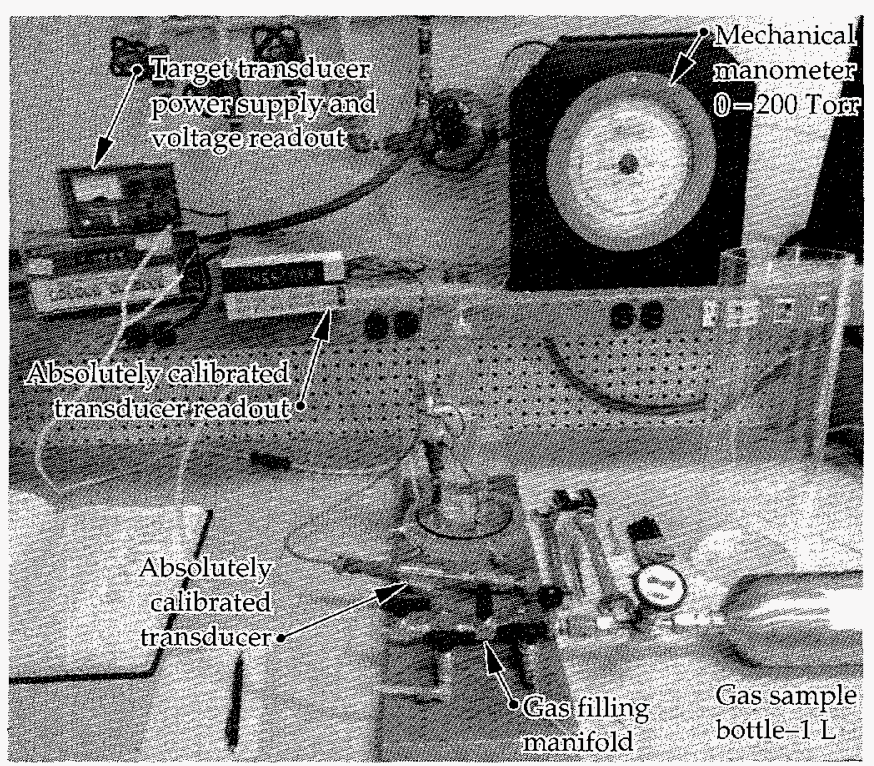

FIGURE 6. Photograph of the evacuation/backfill chamber and gas filling system. $\quad(10-00-0795-1816 \mathrm{pb02})$ 


\section{Summary}

Targets to test the point design for the proposed NIF were developed and fabricated for use on the Nova laser at LLNL. Sub-micrometer-thick polyimide windows capable of holding $>1$ atm were attached to closed-geometry (Nova-hohlraum-style) and opengeometry (nearly spherical gas-bag) targets. Together with the Target Fabrication group at LANL, we fabricated a system to pressure-test targets and to verify gas integrity, prior to delivery to Nova. We also developed a gas-mixing system that permits mixture ratios as low as 0.25 at. \% for individual gases and a system to measure gas pressure inside the target to integrate into the Nova target positioner. In support of the ICF programs at LANL and LLNL, more than 450 gas-cell targets have been successfully fielded on Nova.

\section{Notes and References}

1. L. Powers, et al., Phys. Rev. Lett. 74, 2957 (1995).

2. J. Lindl, "Development of the Indirect-Drive Approach to Inertial Confinement Fusion and the Target Physics Basis for Ignition and Gain," Lawrence Livermore National Laboratory, Livermore, CA, UCRL-JC-119015 (1995).

3. D. H. Kalantar, et al, ICF Quarterly Report 4(4), 145-151, Lawrence Livermore National Laboratory, Livermore, CA, UCRL-LR-105821-94-4 (1994).

4. B. J. MacGowan, C. A. Back, R. L. Berger, et al, "The Study of Parametric Instabilities in NIF-Scale Plasmas on Nova," Lawrence Livermore National Laboratory, Livermore, CA, UCRL-JC-118524 (1995); prepared for the Proceedings of the International Atomic Energy Agency 15th International Conference on Plasma Physics and Controlled Nuclear Fusion Research, Seville, Spain, Sept. 26-Oct. 1, 1995.

5. M. Salazar and the LANL Target Fabrication group are responsible for the original design and fabrication of the evacuation test chamber system and the evacuation backfill method. Their staff is also responsible for fabricating the additional gas handling hardware used for target testing. 


\title{
OPTIMIZATION OF X-RAY SOURCES FROM A HigH- Average-Power ND:Glass LASER-Produced Plasma For Proximity LithograPHY
}

\author{
P. Celliers \\ S. Mrowka \\ D. Matthews \\ L. B. Da Silva \\ M. Norton \\ J.A. Abate* \\ C. B. Dane \\ L. Hackel \\ J. Maldonado ${ }^{\dagger}$
}

\section{Introduction}

The concept of a laser-based proximity lithography system for electronic microcircuit production has advanced to the point where a detailed design of a prototype system capable of exposing wafers at 40 wafer levels per hr is technically feasible with highaverage-power laser technology. In proximity $x$-ray lithography, a photoresist composed of polymethylmethacrylate (PMMA) or similar material is exposed to $x$ rays transmitted through a mask placed near the photoresist, a procedure which is similar to making a photographic contact print. The mask contains a pattern of opaque metal features, with line widths as small as $0.12 \mu \mathrm{m}$, placed on a thin (1- $\mu \mathrm{m}$ thick) Si membrane. During the exposure, the shadow of the mask projected onto the resist produces in the physical and chemical properties of the resist a pattern of variation with the same size and shape as the features contained in the metal mask. This pattern can be further processed to produce microscopic structures in the Si substrate.

The main application envisioned for this technology is the production of electronic microcircuits with spatial features significantly smaller than currently achievable with conventional optical lithographic techniques $(0.12 \mu \mathrm{m}$ vs $0.25 \mu \mathrm{m})$. This article describes work on optimizing a laser-produced plasma $x$-ray source intended for microcircuit production by proximity lithography.

\section{Background}

To obtain the best transmission through the $\mathrm{Si}$ substrate, followed by absorption in the PMMA, the illumination source should occupy a band of x-ray wavelengths somewhere above the Si K-edge (6.74 $\AA$ ) but long enough that the $x$ rays are efficiently absorbed in the PMMA resist layer (possibly up to $15 \AA$ ). The $x$-ray wavelength is short enough that blurring due to $x$-ray diffraction from edges in the pattern will not be significant. Current system designs for proximity lithography require a source with a median emission wavelength in the range of 10-14 $\AA$ and a $20 \%$ bandwidth. The mask can be illuminated with a collimated source of $x$ rays, such as from a synchrotron tuned to operate at the desired wavelengths. In fact, the basic principles of proximity lithography have already been demonstrated using synchrotron sources. ${ }^{1,2}$ However, synchrotron facilities are inherently expensive and therefore not amenable for most circuit manufacturers to acquire and operate.

Laser-produced plasmas have been recognized as a promising alternative to synchrotrons for a number of years. The physical specifications that the plasma source must meet can be summarized as follows: the source must deliver approximately $15 \mathrm{~mJ} / \mathrm{cm}^{2}$ of $\mathrm{x}$-ray fluence to the resist with a uniformity of $1 \%$ over a $3 \times 3 \mathrm{~cm}^{2}$ area within an exposure interval of approximately $1.3 \mathrm{~s}$; the x-ray spectrum must occupy a $20 \%$ bandwidth centered on a wavelength $\sim 12 \AA$. These requirements can be met using a $1-\mathrm{kW}$ laser assuming about $10 \% /(2 \pi \mathrm{sr})$ conversion of laser energy into the desired $x$-ray band; the mask and resist are assumed to be about $50 \mathrm{~cm}$ from the source point to satisfy the uniformity requirements. Variations on these figures may occur depending on a number of design options, but it is clear that in any system a substantial portion of the costs are directly

\footnotetext{
* AT\&T Bell Laboratories, Murray Hill, New Jersey

† IBM Microelectronics, Hopewell Junction, New York
} 
driven by the average power level needed from the laser, which in turn hinges on achieving high $x$-ray yields.

$X$-ray yields from laser-produced plasmas have been investigated in great detail for nearly two decades and have been examined over a wide variety of laser parameters (wavelength, pulse duration, focused intensity) as well as target material and $x$-ray emission wavelengths. ${ }^{3-12}$ Several studies have focused specifically on lithography applications to match target materials with realistic laser parameters to produce the required $\mathrm{x}$-ray spectrum (keV $\mathrm{x}$ rays) at an acceptable yield. ${ }^{13-19} X$ rays from laser-produced plasmas in the $1-\mathrm{keV}$ energy range required for proximity lithography are produced most efficiently with focused laser intensities around $10^{13} \mathrm{~W} / \mathrm{cm}^{2}$ and from targets with atomic numbers in the range $Z=26-30$ (Ne-like ions, L-shell emission) or $Z=53-56$ (Ni-like ions, $\mathrm{M}$-shell emission). As a general finding, $x$-ray yields are known to improve substantially with decreasing laser wavelength. ${ }^{4,5,7}$

$X$-ray yields from laser-produced plasmas also depend on the pulse duration, which determines the characteristic plasma volume achieved during the pulse. High conversion efficiency has been usually observed with moderate-duration pulses (0.5-10 ns). ${ }^{11,16}$ However, it can also be achieved with shorter pulses $(<100 \mathrm{ps})$ in combination with a weak prepulse to generate a long-scale-length plasma. ${ }^{12,20}$ It is not clear from the findings of previous studies whether high conversion efficiency can be achieved from pulses longer than $10 \mathrm{~ns}$. Experiments with 8-ns Nd:glass laser pulses indicate that efficiencies around $8 \% /(2 \pi \mathrm{sr})$ could be achieved. ${ }^{3}$ However, experiments with $30-\mathrm{ns} \mathrm{KrF}$ pulses focused to more than $10^{14} \mathrm{~W} / \mathrm{cm}^{2}$ failed to achieve yields comparable to measurements with shorter pulses at the same wavelength. ${ }^{17}$ Chaker et al. ${ }^{19}$ suggest a practical limit on the pulse duration of less than about $5 \mathrm{~ns}$, but this conclusion is tentative in the absence of experimental data. The requirements of high average power with high focused intensities and moderate pulse duration are potentially in conflict with the pulse parameters of current high-average-power laser technology, which operate more reliably with rather long pulse durations in the range of $10-15 \mathrm{~ns}$ for Nd:glass technology and 25-30 ns for excimer lasers.

One potential laser driver for proximity lithography is a high-average-power $\mathrm{Nd}$ :glass slab design operating with high pulse energy and moderate repetition rate. Currently, the most advanced realization of this technology is available at Lawrence Livermore National Laboratory (LLNL) ${ }^{21}$ and consists of a flash-lamppumped system capable of producing near-diffractionlimited pulses with approximately 13-ns full-width at half maximum (FWHM) duration at energies of $\sim 20 \mathrm{~J}$ at a rate of $6 \mathrm{~Hz}$ to produce an average power of $120 \mathrm{~W}$. The design is scalable to higher average power by increasing the pulse energy and/or repetition rate; we envision average powers approaching $2 \mathrm{~kW}$. An important characteristic of this high-average-power capability is the use of phase conjugate wavefront correction $^{22}$ to ensure a uniform intensity and a near ideal phase front in the final pass of the slab amplifier, which is necessary to ensure reliable operation. This technology operates most effectively with a rather long pulse duration (12-14 ns), which raises the issue of whether the $x$-ray yields produced with this laser can approach the maximum yields observed with shorter pulses. We examined this issue experimentally in the work described in this article.

\section{Experiment}

We measured and optimized the $x$-ray conversion efficiency from several L-shell emitters ( $\mathrm{Fe}, \mathrm{Cu}, \mathrm{Zn}$, brass, stainless steel) and one M-shell emitter (Xe). The studies with Xe examined the solid form using a cryogenic target. In addition to producing high $x$-ray yields at the desired wavelengths, Xe targets offer the potential to design a source with considerably reduced debris generation. We investigated all targets at two operating wavelengths of the laser: the fundamental wavelength, $1.053 \mu \mathrm{m}$, and the second harmonic, $0.527 \mu \mathrm{m}$.

Figure 1 shows the layout for the experiments with solid planar targets. This section describes the five main parts identified by Fig. 1: the laser beam, the solid targets, the charge-coupled device (CCD) camera and spectrometer system, the filtered photoconductive diamond (PCD) detectors, and the pinhole camera.

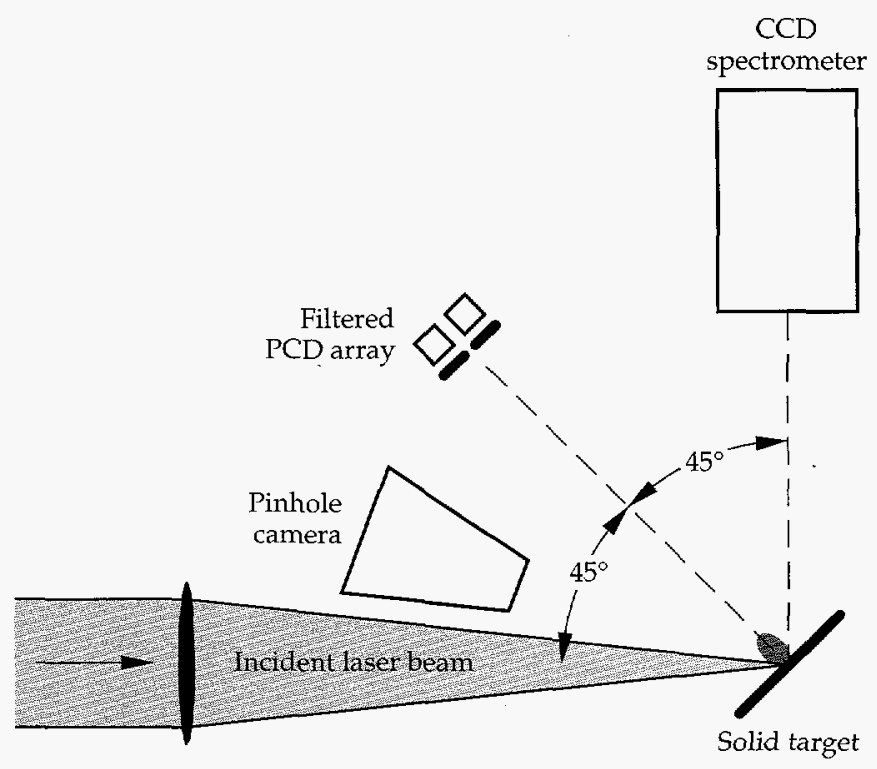

FIGURE 1. Experimental arrangement employed for solid target $\mathrm{x}$-ray yield measurements. (10-06-0595-1100pb01) 


\section{Laser Parameters}

A $330-\mathrm{mm}$ lens focused the laser into the center of the experimental chamber onto a planar target oriented at $45^{\circ}$ to the laser axis. We monitored the laser energy with a fast pyroelectric detector and cross-calibrated it with a calorimetric energy meter. Pulse energy varied from $\sim 1-20 \mathrm{~J}$ at $1.053 \mu \mathrm{m}$ and $\sim 0.5-12 \mathrm{~J}$ at $0.527 \mu \mathrm{m}$. The beam delivered to the target chamber was square in cross-section, measuring $25 \mathrm{~mm}$ per side. We used a $330-\mathrm{mm}$ focal-length lens to produce an $f / 13$ focus. We adjusted the beam focus by translating the lens on a translation stage. In a separate test of the beam quality, we projected a series of far-field beam images with $0.527-\mu \mathrm{m}$ light onto a CCD camera using a well corrected microscope objective. We found that, at best focus, $80 \%$ of the beam energy was contained within a diameter of $27 \mu \mathrm{m}$, equivalent to about $1.5 \times$ diffraction limit. The maximum focused intensities achieved with this beam quality are $7 \times 10^{13} \mathrm{~W} / \mathrm{cm}^{2}$ for $1.053-\mu \mathrm{m}$ light and $2 \times 10^{14} \mathrm{~W} / \mathrm{cm}^{2}$ for $0.527-\mu \mathrm{m}$ light. These peak intensities meet or exceed the intensities needed to obtain good $\mathrm{x}$-ray yields in the kilovolt range.

\section{Solid Targets}

The target mount consisted of an $\mathrm{x}-\mathrm{y}-\mathrm{z}$ translation stage with an aluminum mounting frame for attaching the target. Motion in a plane parallel to the target surface $(x-y)$ allowed us to position a fresh surface of the target in the beam for each shot while maintaining the same axial position relative to the laser focus. All of the solid targets could be mounted (as tapes or thin plates) directly onto the mounting frame. For most of these materials, the target thickness was much larger than typical ablation depths. In the particular case of Fe, however, the target consisted of a thin layer of Fe powder (3-5 $\mu \mathrm{m})$ bonded to a mylar tape substrate. This "mass-limited" target is designed to provide enough material to produce $x$ rays while limiting debris production.

In the particular case of solid $\mathrm{Xe}$, the target apparatus consisted of a 1-mm-thick Cu plate thermally connected with a 2-in-long $\mathrm{Cu}$ braid to a cold finger and cooled to approximately $20 \mathrm{~K}$. By condensing Xe gas onto the cryogenic surface, we produced a thin $(\sim 100 \mu \mathrm{m})$, solid Xe layer on the $\mathrm{Cu}$ substrate. Although the melting point of $\mathrm{Xe}$ (at atmospheric pressure) is around $160 \mathrm{~K}$, a temperature of $20 \mathrm{~K}$ was required in vacuum to maintain a low enough $X e$ vapor pressure to produce a stable condensed layer on the $\mathrm{Cu}$ substrate and to minimize reabsorption of the Xe emission by residual cold Xe gas in the chamber.

\section{Curved Crystal Spectrograph}

To determine $x$-ray yields, we needed accurate measurements of the $x$-ray spectrum produced by each source. The spectra varied not only with target material, but also with the laser parameters (wavelength, pulse energy, and focus). A curved potassium acid pthalate (KAP) crystal spectrograph recorded $x$-ray spectra $90^{\circ}$ relative to the laser axis and $45^{\circ}$ to the target normal, using a high-resolution CCD camera system operating with 16-bit readout resolution. ${ }^{23}$ The spectrometer-three separate KAP crystals bent to the same radius of $79 \mathrm{~mm}$-rested $\sim 350 \mathrm{~mm}$ from the plasma. The detector was a back-illuminated Tektronix TK1024 CCD chip. The system was sensitive enough that all spectra recorded in these experiments were produced by a single laser pulse. To obtain the complete spectrum, we placed the KAP crystals at slightly different standoff distances from the plasma to sample the 9-19-A spectral region in three overlapping segments. To block out visible and UV portions of the spectrum, we placed one or two layers of a light-tight aluminized mylar film ( $5000 \AA \mathrm{Al} / 1.5 \mu \mathrm{m}$ mylar) at the entrance to the spectrograph. This film was subject to occasional damage from target debris; therefore, we checked and replaced it at appropriate intervals.

We calibrated the spectrometer dispersion by identifying known features of the Fe and Cu L-shell spectrum and applying a low-order polynomial mapping from detector position to a wavelength scale. Within the 9-19- $\AA$ band, the CCD array detector responds linearly to the $x$-ray fluence independent of wavelength. Corrections had to be applied to the raw data to account for the filter transmission and the KAP crystal reflectivity. The filter transmission was independently calibrated at an in-house facility to determine its transmission over the 9-19- $\AA$ wavelength band. Henke, et al. previously calibrated the KAP reflectivity. ${ }^{24}$

\section{Absolutely Calibrated PCD and Yield Measurements}

We recorded $\mathrm{x}$-ray yields with a set of four filtered type IIA PCD detectors. ${ }^{25}$ These were mounted in a compact $2 \times 2$ square array $15.9 \mathrm{~cm}$ from the target at an angle approximately normal to the target surface. For all measurements, the PCDs were biased with $600 \mathrm{~V}$, and the signal was coupled through a capacitor into a $50-\Omega$ cable connected to a high-speed digital oscilloscope for recording. These detectors have been used in previous $\mathrm{x}$-ray yield experiments at $\mathrm{LLNL}^{26}$ and have been absolutely calibrated. ${ }^{27}$ Within the 9-19- $\AA$ wavelength band observed in these measurements, the detectors have a flat wavelength response. For the bias conditions and spectral range used in the measurements, the sensitivity of these devices was nominally $7.5 \times 10^{-4}$ $\mathrm{A} / \mathrm{W}$. We assumed an uncertainty of approximately $20 \%$ on this value, as reported in the original absolute calibration. 
Figure 2 shows examples of a PCD signal trace and a trace of the laser pulse recorded on high-speed oscilloscopes. The $\mathrm{x}$-ray pulses displayed the same temporal structure, if any, produced in the laser pulses. The FWHM pulse duration of the x-ray signals observed from solid targets was similar to, but somewhat shorter than, the laser pulse by an amount $\sim 20-30 \%$. Hence the $\mathrm{x}$-ray pulses from solids were typically $10 \mathrm{~ns}$ FWHM, while the laser pulse was 12-14 ns FWHM.

We monitored contributions from various parts of the $x$-ray spectrum using a set of four different filters on the PCD detector array. The filter set was designed to sample the 8-20- $\AA$ band in three intervals: a 10.6- $\mu \mathrm{m}$ Al filter (8-12 $\AA$ ), a $2-\mu \mathrm{m}$ Zn filter (12-16 $\AA$ ), and a $2-\mu \mathrm{m}$ Co filter $(16-20 \AA)$. The fourth channel used an aluminized mylar filter to sample most (8-20 $\AA$ ) of the spectrum. Prior to all measurements, we performed a cross-calibration of the individual PCD sensitivities. We did this by recording the signals from each detector using 2- $\mu \mathrm{m} \mathrm{Zn}$ filters on each and with all detectors simultaneously illuminated by $x$ rays from an Fe or type 302 stainless steel (SS302) laser-produced plasma. We took several data points for each of three rearrangements of the individual $\mathrm{Zn}$ filters so that we could eliminate effects due to variability in the filter transmission. We found variation in detector response among the four devices consistent with a $\pm 20 \%$ spread in sensitivity. The extracted filter transmissions were consistent with a $10-15 \%$ variability from one piece to the next. Based on these uncertainties alone, the absolute uncertainty in yield for these measurements is around $\pm 25 \%$. Relative uncertainties in comparing different target materials or laser parameters are much better, around $10 \%$, determined primarily by shot-to-shot variations.

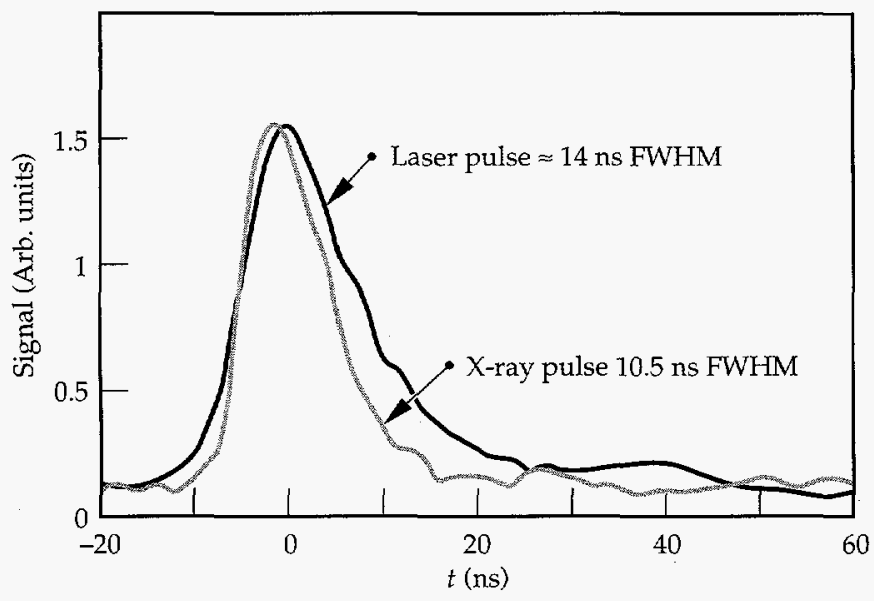

FIGURE 2. Sample oscilloscope traces of the laser pulse measured with a photodiode and an x-ray pulse measured with a photoconductive diamond (PCD) detector. Vertical scales and temporal offsets for both pulses are arbitrary, and were adjusted for comparison. (10-06-0595-1101pb01)
To determine the absolute $x$-ray yield, we integrated the recorded oscilloscope signals to produce a value proportional to the total x-ray fluence striking the detector. We then converted this raw data value to an absolute measure of the $x$-ray fluence by factoring in corrections for detector sensitivity, solid angle, filter transmission, and the emission spectrum. Simultaneous measurement of the spectrum is crucial for an accurate determination of the fractional weight of the spectrum viewed by each channel. We determined $x$-ray yields by multiplying each detector signal by a factor inversely proportional to the known filter response multiplied by the measured spectrum at each incident energy. We then obtained the resulting conversion efficiency from an average of the contributions measured from each. channel and the known input laser energy.

The CCD detector on the spectrometer also provided an accurate method of assessing relative $x$-ray yields (from one target to the next, or for changes in other parameters, such as laser energy). This provided us with a cross-check against the yields inferred on the basis of the PCD measurements. We found good agreement between the relative yields determined from integrating the spectrum recorded on the CCD and the signals measured with the PCD array.

\section{Pinhole Camera}

We monitored plasma source size with an $\mathrm{x}$-ray pinhole camera coupled to a video CCD and a computercontrolled readout. We placed the pinhole about $3 \mathrm{~cm}$ from the target and operated at a magnification of 2-3. Because it was filtered with $18-\mu \mathrm{m}$ Al foil, the $x$-ray spectrum was sensitive mostly to the $8-12-\AA$ portion of the emitted spectrum.

\section{Methods and Results}

This section provides specific details about methods and results for the experiment. We divide the section into four subsections: focus optimization, yield variation with pulse energy, x-ray spectra, and angular distribution.

\section{Focus Optimization}

For each target type and laser wavelength investigated, we optimized and measured the $x$-ray yield with a standard procedure consisting of two parts. First, we did a focal scan consisting of a series of shots examining yield as a function of axial lens position at maximum laser energy ( $20 \mathrm{~J}$ at $1.053 \mu \mathrm{m}$ and $12 \mathrm{~J}$ at $0.527 \mu \mathrm{m}$ ). From this procedure, we identified the lens position corresponding to maximum yield as determined by the PCD measurements. Second, with the lens fixed at the maximum yield position, we systematically reduced the laser energy (described in the next section). 
Figure 3 shows an example of the variation of x-ray yield with lens position for $\mathrm{Cu}$ and type SS302 irradiated with 1.053- $\mathrm{mm}$ laser light. We found in general that the $x$-ray yield reached a broad maximum within $\pm 1 \mathrm{~mm}$ of the lens focus. The position of optimum conversion was largely independent of the target material, varying by an amount of approximately $\pm 0.5 \mathrm{~mm}$ for different targets.

Figure 4(a) shows pinhole photographs of the x-ray source region throughout the focal scans for $1.053-\mu \mathrm{m}$ irradiation. The double-lobed structure apparent at lens positions of 14 and $15 \mathrm{~mm}$ originates from the intensity distribution in the square beam as it approaches the focus. Similar lobed structures were also evident in

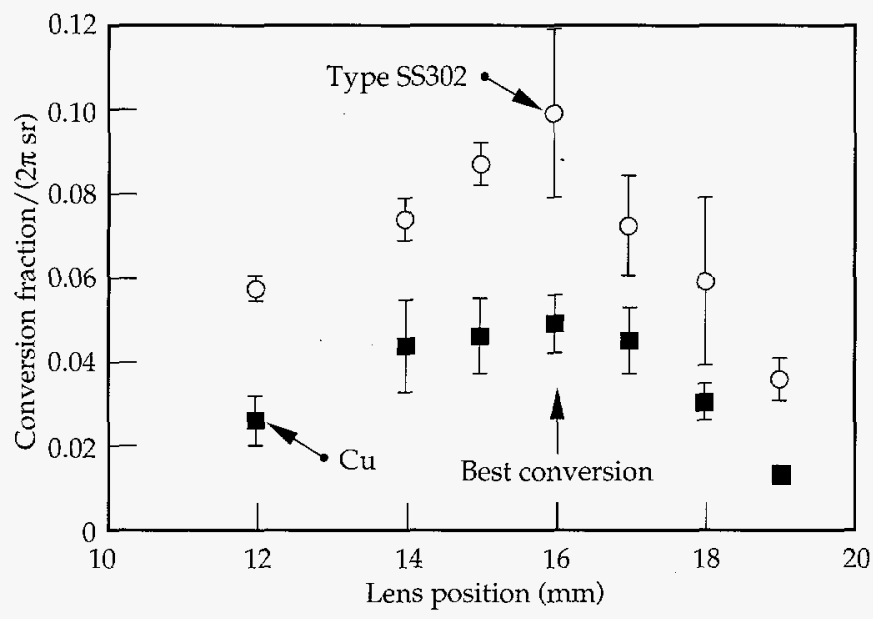

FIGURE 3. Variation of $x$-ray yield with focusing lens position for 1.053- $\mu \mathrm{m}$ laser light; we used this procedure to optimize the yield for a given target type. (10-06-0595-1102pb01) the 0.527- $\mu \mathrm{m}$ laser-produced plasmas. Analyzing these images, we assume that the size of the x-ray emitting region correlates with the spatial extent of the laser intensity distribution illuminating the plasma. Figure 4(b) shows the variation of $\mathrm{x}$-ray source size measured in these focal scans. The emitting region of the plasma was considerably larger than the beam diameter close to best focus since under no conditions did we observe an $x$-ray source region smaller than about $150 \mu \mathrm{m}$ in diameter, a size $3-5$ times larger than the beam diameter expected at best focus for the $1.5 \times$ diffraction limited beam. We took the lens position corresponding to best focus to be the point where the observed source diameter reached a minimum. The geometrical extent of the focal cone for the $f / 13$ focus is also displayed in these figures for comparison with the data.

The most important information from these sequences is an assessment of the size of the x-ray emitting region in the source and an approximate idea of the laser intensity illuminating the plasma at these optimum positions. At the lens position corresponding to optimum $x$-ray yield, the diameter of the source region evident from the pinhole images was around $280 \mu \mathrm{m}$ for $1.053-\mu \mathrm{m}$ laser light and $240 \mu \mathrm{m}$ for $0.527-\mu \mathrm{m}$ light. It is also evident that the focal cone of the $f / 13$ focus is smaller than the plasmas at most positions where significant $x$-ray emission was observed, and that the lens position corresponding to best yield does not correspond to the position of best focus, although it was much closer to best focus for $0.527-\mu \mathrm{m}$ light than for 1.053- $\mu \mathrm{m}$ light. (X-ray yield on the converging side of the $1.053-\mu \mathrm{m}$ focused beam should be similar to the diverging side; for all measurements reported here the beam was focused on target with a diverging focus.) Average (a)

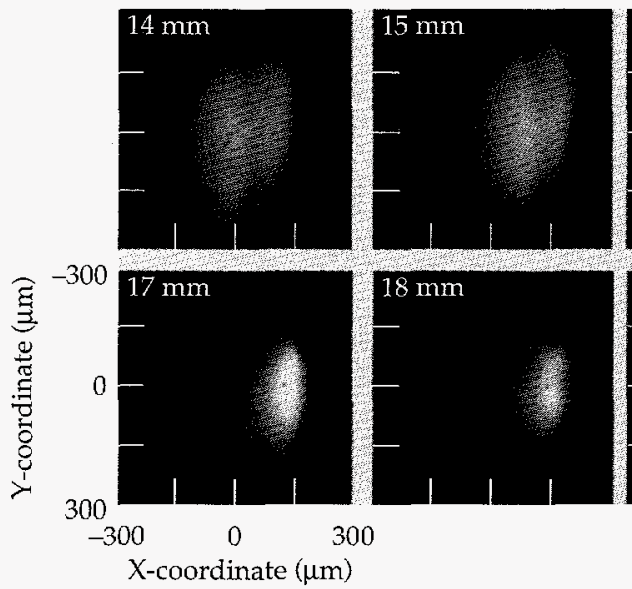

(b)
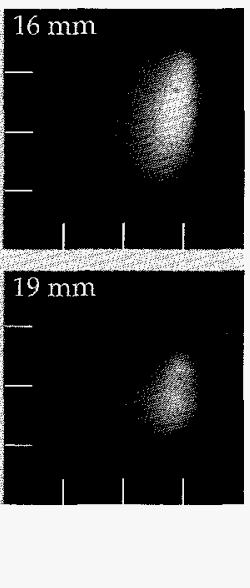

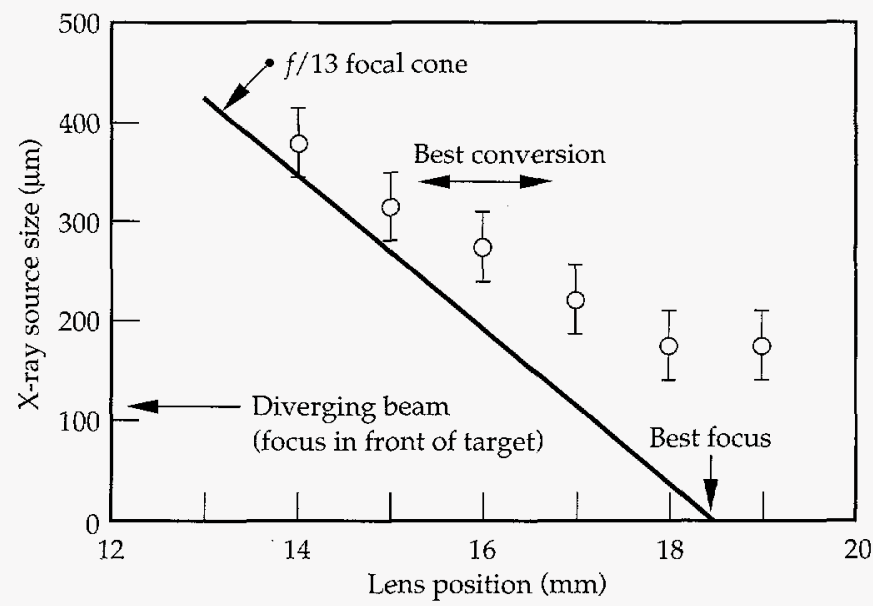

FIGURE 4. Variation of $x$-ray emitting plasma volume with focusing lens position for $1.053-\mu \mathrm{m}$ laser light irradiating a solid Fe target. The origin of the lens position scale is arbitrary. (a) A sequence of pinhole photographs of the plasma x-ray emission taken at various lens positions (indicated on the individual frames). (b) Variation of plasma size with lens position measured from the pinhole photographs. Also shown for comparison is the beam size assuming a geometric $f / 13$ focal cone with best focus assumed to occur at the lens position producing the smallest plasma volume. (10-06-0595-1103pb01) 
beam intensities illuminating the plasma at these lens positions can be estimated from the geometrical extent of the focal cone and the distance of the optimum lens position from best focus. For 1.053- $\mu \mathrm{m}$ light, the beam diameter at optimum focus was $\sim 190 \mu \mathrm{m}$, to produce a beam intensity $\sim 5 \times 10^{12} \mathrm{~W} / \mathrm{cm}^{2}$. For $0.527-\mu \mathrm{m}$ light, the beam diameter was approximately $100 \mu \mathrm{m}$, a factor of two smaller than for the optimum 1.053- $\mu \mathrm{m}$ situation, producing intensities $\sim 1.2 \times 10^{13} \mathrm{~W} / \mathrm{cm}^{2}$.

\section{Yield Variation with Pulse Energy}

After we determined the optimum focus position, we fixed the lens at its optimum position and measured $x$-ray yields while the laser energy was varied throughout the available range below the maximum setting. We easily adjusted laser energy by varying a waveplate within the preamplifier chain to control the pulse energy prior to the final amplifier passes. Both the temporal pulse shape and spatial beam parameters (focus position) were unaffected by this adjustment.

An important characteristic of all of the measurements from planar targets measured in this research was an increasing $x$-ray yield with pulse energy. For all materials, the conversion increased monotonically from near zero at low pulse energies and increased to a saturation value before leveling off. Figure 5 shows an example of this dependence for (a) type SS302 and (b) $\mathrm{Cu}$. Within the energy range available there was no evidence of a regime where conversion fraction decreased with increasing pulse energy. The saturation value varied with the target material and the laser wavelength. With type SS302 (median emission wavelength at $15 \AA$ ), the laser energy at saturation is clearly lower than for $\mathrm{Cu}$ (median emission wavelength at $11.5 \AA$ ). In the case of $\mathrm{Cu}$, it is not clear that the dependence of $x$-ray yield with energy has reached a final saturation level at the maximum laser pulse energies available in these experiments, although saturation appears to be $\sim 5-10 \mathrm{~J}$ for type SS302. Correlated with the pulse energy dependence of yield was a clear shift in the $x$-ray spectrum for any given target to shorter wavelengths (harder photons) with increasing pulse energy. We expected this due to the fact that increasing pulse energies produce higher intensities and drive hotter plasmas.
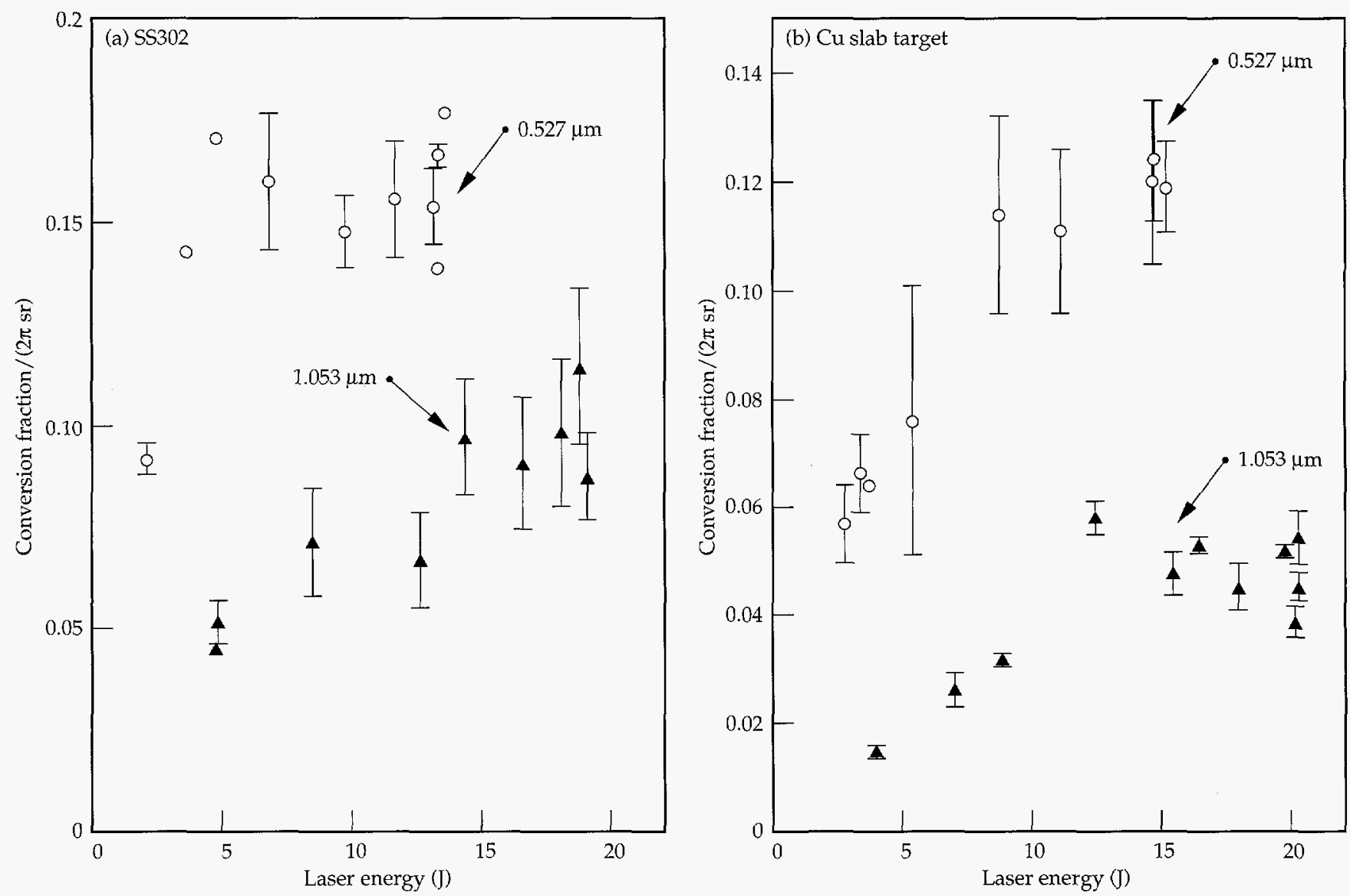

FIGURE 5. Pulse energy dependence of $x$-ray yield for two target materials and both drive wavelengths. Target materials are (a) type SS302 and (b) Cu. (10-06-0595-1104pb01) 


\section{X-Ray Spectra}

Figure 6 shows a catalogue of spectra for all of the materials tested using $0.527-\mu \mathrm{m}$ laser light. Also identified on the spectra are integral curves depicting the integrated conversion fraction through the short to long range of emission wavelengths. We observed a similar set of spectra using 1.053- $\mu \mathrm{m}$ light, with the main difference being a shift in the distribution of emission to longer wavelengths within the characteristic spectrum of each material. For the materials selected, Fig. 7 summarizes the $x$-ray wavelength range (10-15 $\AA$ ).

All of the Xe spectra exhibit a bilobed distribution of emission with a main component emitting at wavelengths from $10-15 \AA$, and a second component from $17-20 \AA$. We also included this latter component, accounting for about $20-30 \%$ of the emitted energy, in our yield determination, although the component is of little use for lithography applications. Excluding this component (i.e., excluding wavelengths $>16 \AA$ ), the $\mathrm{Xe}$ conversion measured with 1.053-light is less than $10 \%$, but still comparable to the type SS302 conversion.

With $0.527-\mu \mathrm{m}$ light the Xe spectrum becomes harder, shifting to shorter wavelengths, and the total conversion improves to around $12 \%$.

The conversion efficiency measured with type SS302 is significantly higher than with pure Fe. We can attribute this largely to the addition of significantly more spectral lines with contributions from $\mathrm{Cr}$, $\mathrm{Mn}, \mathrm{Ni}$, etc., present in the stainless steel alloy. Figure 6(b) demonstrates this, showing the dense spectrum of stainless steel as compared with the pure Fe spectrum in Figure 6(c). We discuss this improved conversion efficiency later in the article.

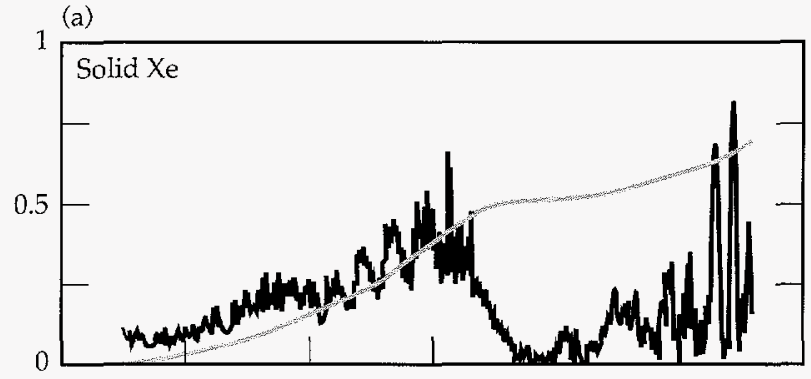

(d)
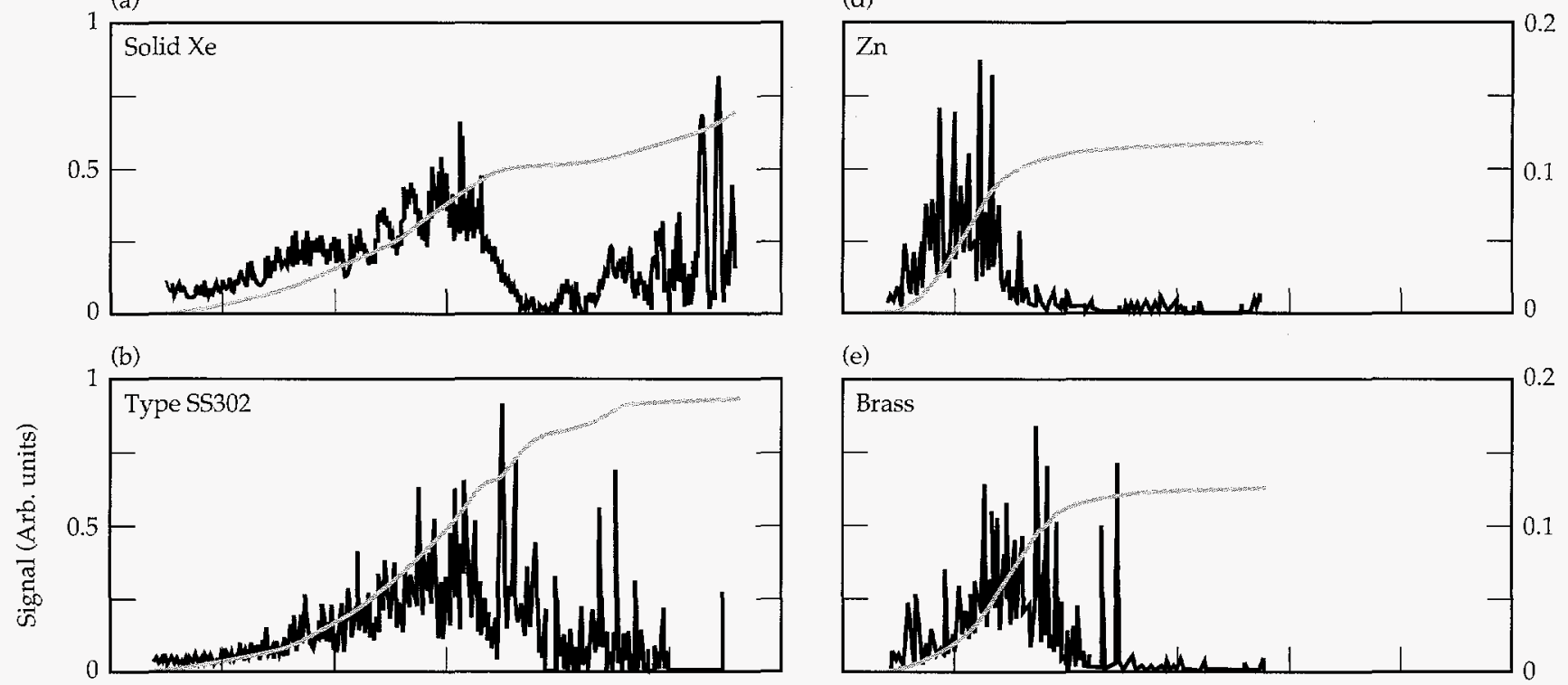

(e)
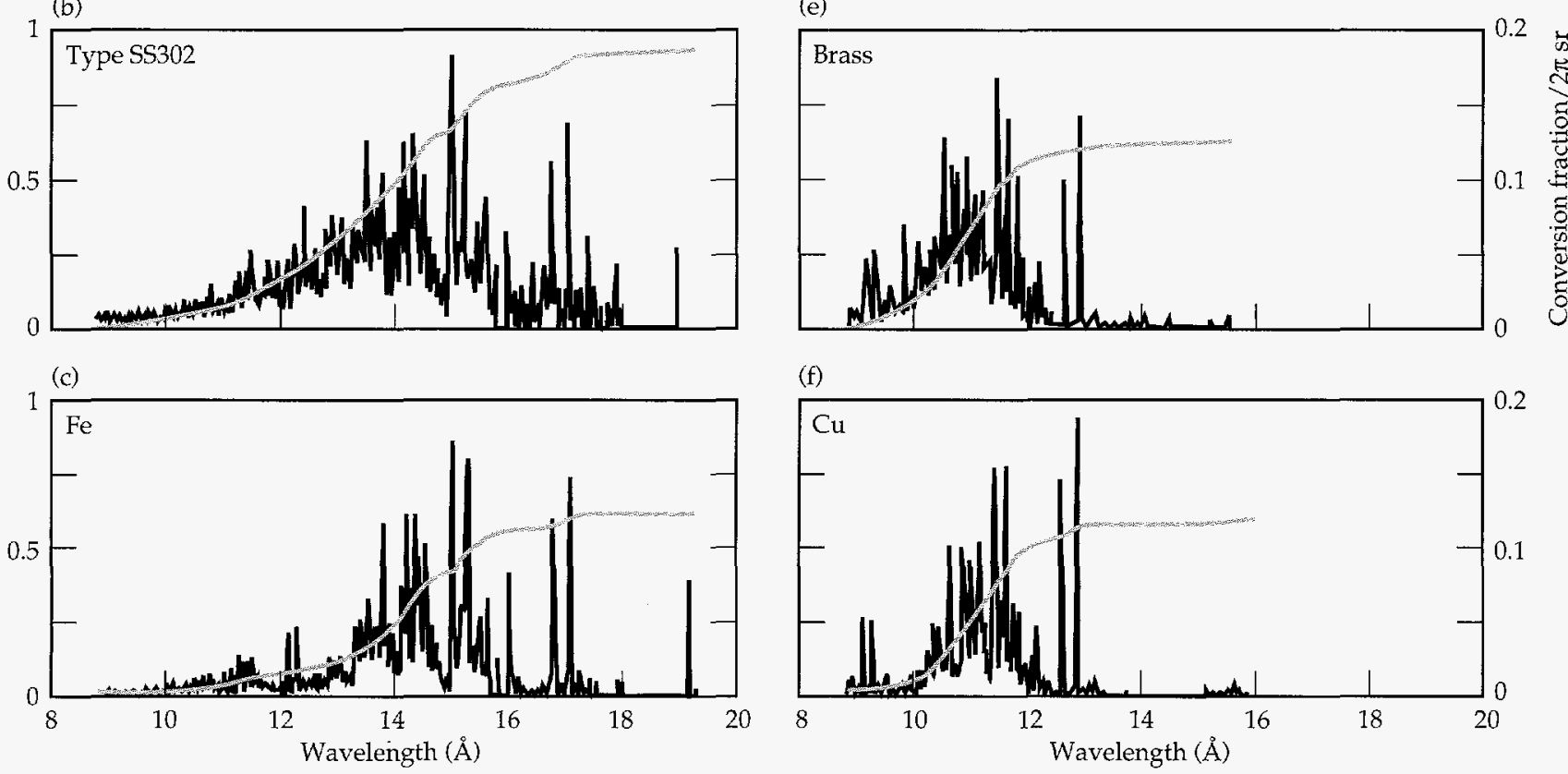

FIGURE 6. X-ray spectra measured for all solid targets using 0.527- $\mathrm{mm}$ laser light. Spectral intensity is displayed on the arbitrary left hand scale; the wavelength-integrated $x$-ray yield for each material can be measured against the right hand scale. Conversion fraction indicates the ratio of x-ray energy to incident laser energy. Target materials are (a) solid Xe, (b) type SS302, (c) Fe, (d) Zn, (e) brass, and (f) Cu. (10-06-0595-1105pb01) 


\section{Angular Distribution}

For planar targets, we also measured the angular distribution of $x$-ray yield. We recorded all yield measurements with the PCD array at target normal, where the yield is expected to reach a maximum. For historical reasons, and to ease comparison with other work, we report the $x$-ray yield as the conversion fraction into $2 \pi \mathrm{sr}$ as if the angular distribution were isotropic and equal to the angular fluence measured at target normal. In general, the angular fluence varies relative to target normal and depends on target material, laser wavelength, laser pulse duration, and possibly other factors. The yield is generally reduced away from target normal and can be fitted with a $\cos ^{\alpha} \theta$ distribution, where $\alpha \leq 1$, and the total conversion into $2 \pi$ s $r$ is less than the fraction reported in this work. There may be reasons to place the lithography exposure system at a location other than target normal; for example, to mitigate against debris, which is also maximized at target normal. Thus an assessment of the angular distribution is important to ascertain any reduction in x-ray yield at other angles.

We did the angular distribution measurements for Fe and type SS302 targets at the optimized conditions for both wavelengths. We fitted all PCD detectors with 2- $\mu \mathrm{m} \mathrm{Zn}$ filters, which transmit the main component of the Fe spectrum. We arranged the detectors in four angular positions in the plane of the laser beam spanning angles from $18-75^{\circ}$ from the target normal. We took several sets of shots for each measurement, for which the $\mathrm{Zn}$ filters were rotated through the detectors, to eliminate effects due to variations in the filter transmissivities. We discuss results from these measurements in the next section.

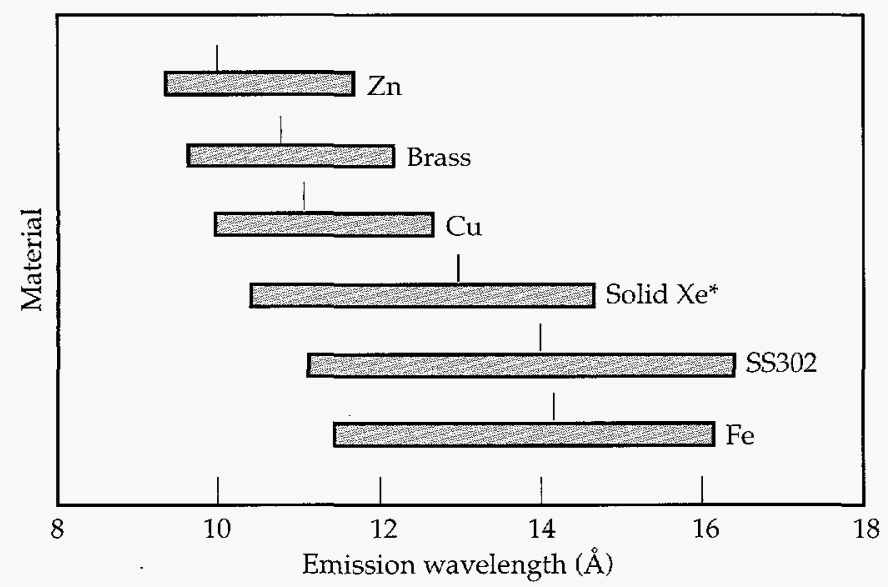

FIGURE 7. Emission wavelengths determined from the spectra measured for all the solid elements using $0.527 \mu \mathrm{m}$ laser irradiation. The bar end points indicate wavelengths spanning 10 to $90 \%$ of the wavelength-integrated emission (i.e., containing $80 \%$ of the emission). The points within the bars indicate the median emission wavelengths (50\% point on the integral curve) for each material. *In the special case of Xe, this evaluation was restricted to wavelengths less than $16 \AA$, which eliminates the long-wavelength emission component $(\sim 18 \AA)$ from the weighting process. $\quad(10-06-0595-1108 \mathrm{pb} 01)$

\section{Discussion}

There are four main areas of discussion for this experiment: $x$-ray yields and spectra, pulse energy dependence, laser-drive wavelength dependence, and angular distribution.

\section{X-Ray Yields and Spectra}

Figure 8 summarizes $x$-ray yields from all of the solid targets, and compares the optimized yield for each material at both wavelengths. We obtained high x-ray yields using the longer wavelength emitters $(\sim 14 \AA)$ with 1.053- $\mu \mathrm{m}$ laser-drive wavelength, the best examples of these being type SS302 and cryogenic Xe. Conversion efficiency of these targets was approximately $10 \% /(2 \pi \mathrm{sr})$ at the target normal. Conversion for shorter wavelength emitters using $1.053-\mu \mathrm{m}$ was significantly less, dropping to $<4 \% /(2 \pi$ sr) for $\mathrm{Zn}$ targets emitting at around $10.5 \AA$. We observed a significant improvement in x-ray conversion with $0.527-\mu \mathrm{m}$ light, where conversion efficiencies for all targets was $\sim 12 \% /(2 \pi \mathrm{sr})$ or better. For the range of shorter-wavelength emitters, including $\mathrm{Cu}$, brass, and $\mathrm{Zn}$, this represents a factor of 3-4 improvement in the yield measured at target normal. Since the energy conversion efficiency for doubling the laser-light frequency is expected to be around $80 \%, 21$ this result indicates that operation at $0.527 \mu \mathrm{m}$ will provide a substantial improvement in conversion of energy from the fundamental laser wavelength to $x$ rays in the 10-12- $\AA$ band using a frequency-doubled laser.

Solid Xe offers the attractive possibility of building a reduced-debris source. The Xe spectrum contains two main components, with a longer-wavelength portion occupying the $17-20 \AA$. For lithography, the $75-80 \%$ energy portion of the spectrum emitted in the 10-14 $\AA$ band is the most useful part. With $0.527-\mu \mathrm{m}$ laser irradiation, this portion of the spectrum is significantly

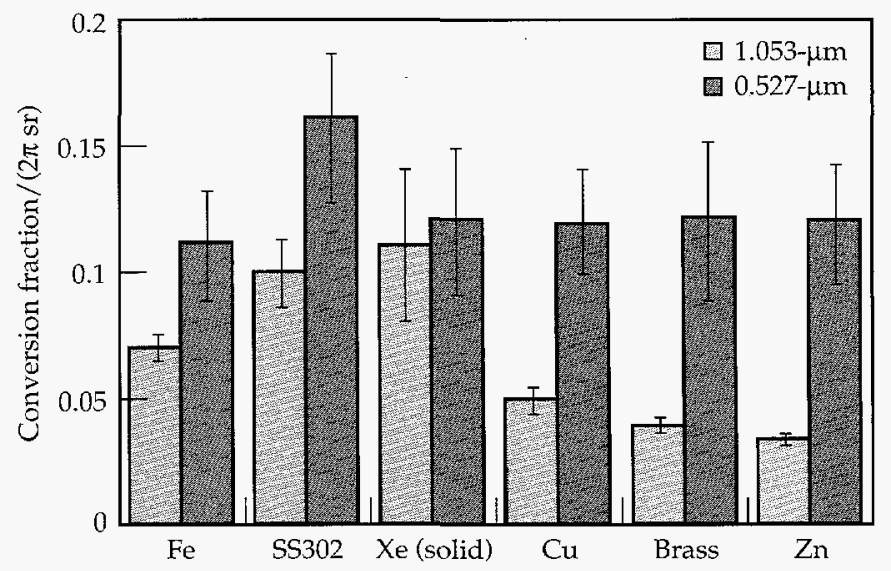

FIGURE 8. Optimized $x$-ray yields measured for all solid targets and both drive wavelengths. (10-06-0595-1107pb01) 
harder than with 1.053- $\mu$ m irradiation. Solid Xe provides $\mathrm{x}$-ray yields comparable to, or better than, other solid targets at both drive wavelengths investigated. In the form of cryogenic pellets, frozen Xe offers the potential for producing a reduced-debris source with yields comparable to other solid target yields.

We attained the best yields with type SS302 targets. The improvement in conversion efficiency compared with pure Fe targets is apparent with $1.053-\mu \mathrm{m}$ laser light, and becomes quite dramatic with $0.527-\mu \mathrm{m}$ light, where the optimum conversion efficiency exceeds $16 \%$ for type SS302. Stainless steel is an alloy comprised mainly of several transition metals with atomic numbers from $Z=24$ through $Z=28$ (the chemical composition of type 302 is approximately $70 \% \mathrm{Fe}, 18 \% \mathrm{Cr}, 9 \% \mathrm{Ni}$, and $2 \% \mathrm{Mn}$ ). These elements emit kilovolt radiation efficiently in the plasmas produced by the laser. The increased yield from the alloy occurs because the strongly emitting lines characteristic of each element are optically thick, or nearly so in these high-density plasmas produced with solid targets; thus, the reduced concentration of Fe found in stainless steel as compared with pure $\mathrm{Fe}$ targets does not lead to a noticeable reduction in the intensity of the characteristic Fe spectral components, while the other alloyed elements in stainless steel provide emission features that fill the gaps in the spectrum. This result indicates the possibility for improving $x$-ray yields by designing mixtures of elements that emit in the desired wavelength band. These will produce high yields by filling in the spectrum with more lines. The high yields of $X e$ are produced for a similar reason, namely that the much more complicated electronic structure of the Xe M-shell provides many more emission lines to fill in the spectral band than the simpler L-shell emitters.

\section{Pulse Energy Dependence}

The pulse energy dependence of the observed x-ray yield is most easily understood in light of previous 0.5-ns pulse duration work by Chaker et al. ${ }^{16}$, in which the conversion efficiency into kilovolt $x$ rays from $\mathrm{Cu}$ targets was observed to drop abruptly below intensities of $\sim 5 \times 10^{12} \mathrm{~W} / \mathrm{cm}^{2}$ for $1.06-\mu \mathrm{m}$ light. Optimum conversion efficiencies for $\mathrm{Cu}$ measured in this work are comparable to the values reported by Chaker et al. As noted, our procedure for optimizing the conversion by scanning the focus automatically places the irradiance near this saturation intensity and not necessarily at the best focus. Consequently, the measurements at decreasing pulse energy will produce lower than optimum intensities, and therefore produce lower x-ray yields. An implication of this interpretation is that it may be possible to attain high yields at lower pulse energies using a faster focusing lens (e.g., $f / 4$ instead of $f / 13$ ) that achieves $\sim 10^{13} \mathrm{~W} / \mathrm{cm}^{2}$ within the beam focus. However, we stress that achieving $10^{13} \mathrm{~W} / \mathrm{cm}^{2}$ is necessary but may not be sufficient to achieve high yields (with long pulses) - this possibility needs to be explored experimentally. In our research, we made no attempts to exploit this method of obtaining high conversion efficiency at lower pulse energies.

\section{Laser-Drive Wavelength Dependence}

As a general rule, the conversion fraction (laser energy to $\mathrm{x}$-ray energy) into $2 \pi \mathrm{sr}$ is higher at $0.527 \mu \mathrm{m}$ than at $1.053 \mu \mathrm{m}$. The phenomenon is most accentuated for the short wavelength (10-12- $\AA$ ) emitters $(\mathrm{Cu}, \mathrm{Zn}$, and brass), where the conversion fraction improves by a factor of 3-4 times over the $1.053-\mu \mathrm{m}$ result. This dramatic improvement in conversion for the shorterwavelength emitters using frequency-doubled laser light more than compensates for losses introduced by converting the fundamental to the second harmonic, and provides a means to obtain high $x$-ray yields at a range of desired wavelengths from $10.5 \AA(\mathrm{Zn})$ to $15 \AA$ (SS302) with a single laser driver.

As increasing pulse energy causes spectral shifts, changing the drive wavelength from 1.053 to $0.527 \mu \mathrm{m}$ clearly caused the spectra of the various target types to shift to shorter wavelengths. This spectral dependence is consistent with (1) the improved coupling of the laser light into plasma heating at higher densities and (2) the improved conversion. Figure 9 shows an example of this spectral shift for solid Xe targets. We observed similar laser-drive-wavelength-dependent spectral shifts for the other materials investigated.

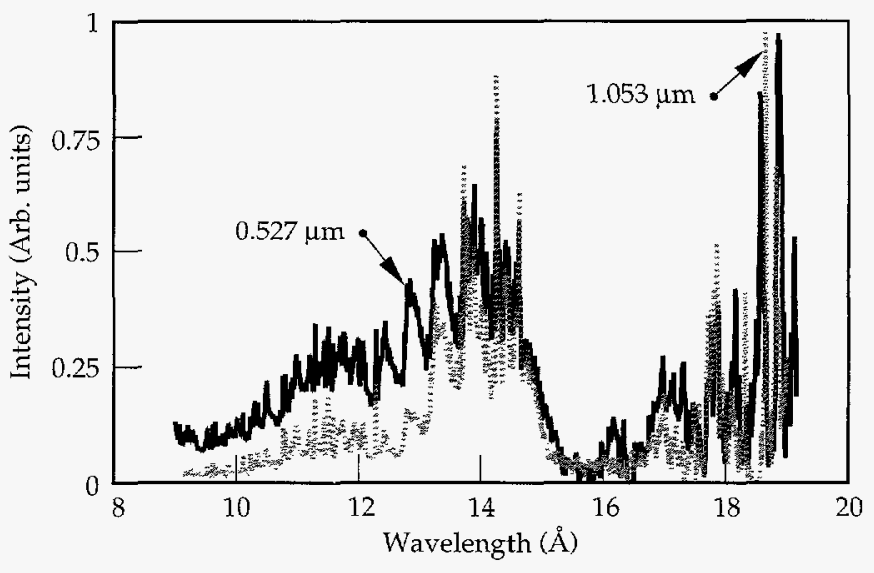

FIGURE 9. Comparison of x-ray spectra from solid Xe measured from plasmas produced with $1.053-\mu \mathrm{m}$ (dashed line) and $0.527-\mu \mathrm{m}$ (solid line) laser light. $\quad(20-07-0695-1659 \mathrm{pb01})$ 


\section{Angular Distribution}

Figure 10 shows the angular distributions measured for both drive wavelengths irradiating Fe or type SS302 targets. Fits to a $\cos ^{\alpha} \theta$ distribution yielded $\alpha \approx 0.2$ for $1.053-\mu \mathrm{m}$ irradiation and $\alpha \approx 0.6$ for $0.53-\mu \mathrm{m}$ irradiation. Neither of these angular distributions is Lambertian $(\alpha=1)$. At $1.053 \mu \mathrm{m}$, the emission is very close to being isotropic, such that at angles of $60-70^{\circ}$ from the target normal, the observed yield remains at $80 \%$ or more of the peak yield at target normal. At $0.53 \mu \mathrm{m}$ the emission is closer to Lambertian, with the yield reduced to half of the peak at angles of $>65^{\circ}$ off target normal. The difference in angular distribution reflects the difference in laser light coupling between the two wavelengths. The shorter wavelength couples much more efficiently into higher density plasma layers, which are optically thick and closer to the target plane, thus producing a physical situation that is close to that of a planar, optically thick Lambertian surface emitter.

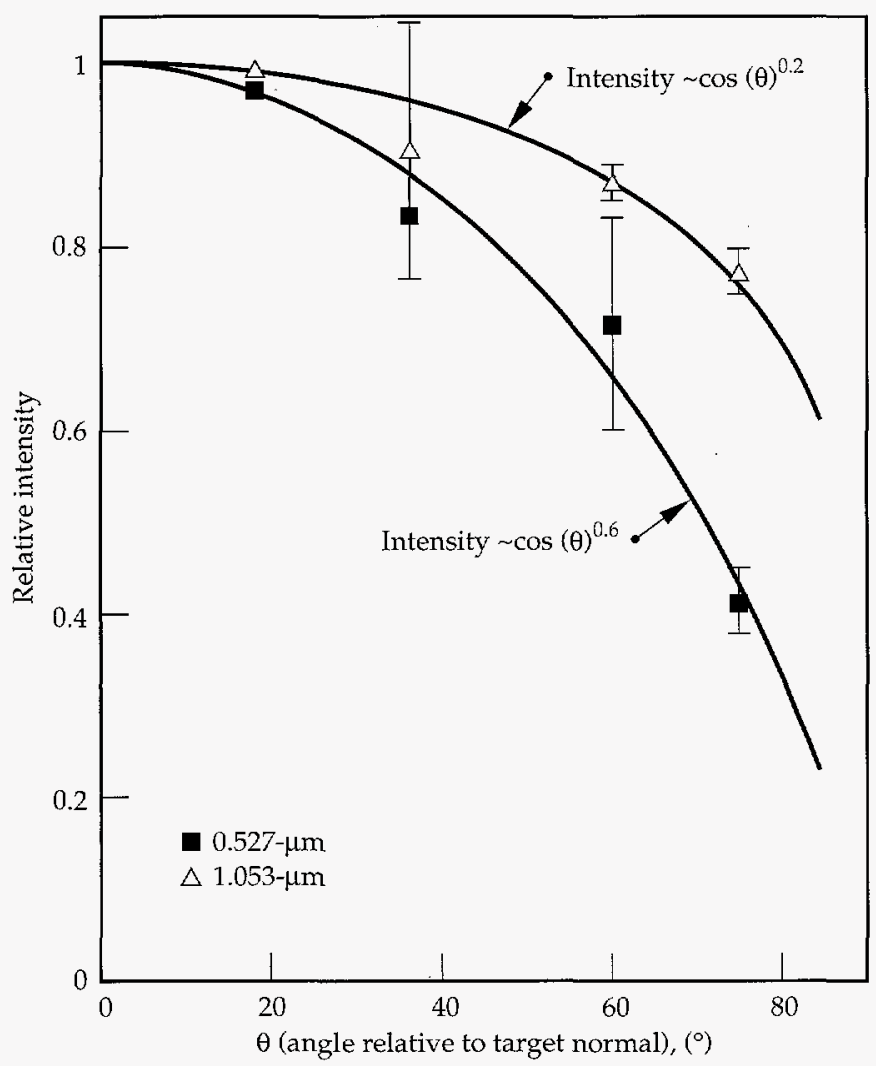

FIGURE 10. Angular distribution of $\mathrm{x}$-ray emission relative to target normal measured for $14-\AA$ emitters Fe or type SS302 at both drive wavelengths. (10-06-0595-1106pb01)

\section{Conclusions}

The intended operating $x$-ray wavelength of a proximity lithography system will be determined by a number of considerations beyond the scope of the present study. For most situations, $x$-ray wavelengths spanning the range from 10-15 $\AA$ may be used. The materials selected in this study embrace this wavelength region. These results demonstrate a significant degree of flexibility in delivering $x$-ray energy within a desired wavelength band using a laser-produced plasma.

$\mathrm{X}$-ray conversion efficiency from all targets increased with increasing laser pulse energy from small values at low pulse energies $(<5 \mathrm{~J})$. A minimum pulse energy of $\sim 5-10 \mathrm{~J}$ was necessary to approach the yields close to the optimum. Above $\sim 10 \mathrm{~J}$, the yield vs pulse energy curve begins to saturate. This behavior is consistent with the observed saturation of conversion with laser intensity observed by Chaker et al. ${ }^{16}$ using 0.5 -ns pulses, thus indicating that the 10-ns plasma behaves similarly in this pulse energy range. At optimum conditions, the average intensity illuminating the plasma was approximately $5 \times 10^{12} \mathrm{~W} / \mathrm{cm}^{2}$ for $1.053-\mu \mathrm{m}$ light, and $1 \times 10^{13} \mathrm{~W} / \mathrm{cm}^{2}$ for $0.527-\mu \mathrm{m}$ light. For both wavelengths the source diameter at optimum conversion was $\sim 250-300 \mu \mathrm{m}$.

The x-ray conversion efficiencies produced with the long-pulse laser driver used in these experiments matched efficiencies measured previously with shorter (nanosecond-duration) pulses. The duration of the $x$-ray pulse matched the duration of the laser pulse, indicating that, at least in the 12-14-ns range, the long pulse duration does not degrade the conversion efficiency. A frequency-doubled Nd:glass laser driver can be used to produce a source with median emission wavelength anywhere from 10.5 to $15 \AA$ at $x$-ray yields that meet the needs of a proximity lithography production system.

\section{Acknowledgments}

We acknowledge technical support from Jim Wintemute for operating the laser, as well as Jim Cox and Ken Haney for fabrication and assembly of the target facility, and Joe Smith for filter calibiations. This work was supported by a contract from the U.S. Department of Defense Advanced Research Projects Agency. 


\section{Notes and References}

1. J. Warlaumont, J. Vac. Sci. Technol. B7, 1634 (1989).

2. G. Zwicker, W. Windbracke, H. Bernt, D. Friedrich, H.-L. Huber, et al., I. Vac. Sci. Technol. B7, 1642-1647 (1989).

3. K. M. Gilbert, J. P. Anthes, M. A. Gusinow, M. A. Palmer, et al., J. Appl. Phys. 51, 1449-1451 (1980).

4. B. Yaakobi, P. Bourke, Y. Conturie, J. Delettrez, J. M. Forsyth, et al., Opt. Commun. 38, 196-200 (1981).

5. H. Nishimura, F. Matsuoka, M. Yagi, K. Yamada, et al., Phys. Fluids 26, 1688-1692 (1983).

6. D. L. Matthews, E. M. Campbell, N. M. Ceglio, G. Hermes, R. Kauffman, et al., T. Appl. Phys. 54, 4260-4268 (1983).

7. W. C. Mead, E. M. Campbell, K. G. Estabrook, R. E. Turner, W. L. Kruer, et al., Phys. Fluids 26, 2316-2331 (1983).

8. P. Alaterre, H. Pépin, R. Fabbro, and B. Faral, Phys. Rev. A 34, 4184-4194 (1986).

9. T. Mochizuki, T. Yabe, K. Okada, M. Hamada, N. Ikeda, S. Kiyokawa, and C. Yamanaka, Phys. Rev. A 33, 525-539 (1986).

10. R. Popil, P. D. Gupta, R. Fedosejevs, and A. A. Offenberger, Phys. Rev. A 35, 3874-3882 (1987).

11. K. Eidmann and W. Schwanda, Laser and Particle Beams 9, 551-562 (1991).

12. J. N. Broughton and R. Fedosejevs, Appl. Phys. Lett. 60, 1818-1821 (1992).

13. D. J. Nagel, M. C. Peckerar, R. R. Whitlock, J. R. Greig, and R. E. Pechacek, Electron. Lett. 14, 781-782 (1978).

14. B. Yaakobi, H. Kim, J. M. Soures, H. W. Deckman, and J. Dunsmuir, Appl. Phys. Lett. 43, 686-688 (1983).
15. H. Pépin, P. Alaterre, M. Chaker, R. Fabbro, B. Faral, et al., J. Vac. Sci. Technol. B 5, 27-32 (1987).

16. M. Chaker, H. Pépin, V. Bareau, B. Lafontaine, I. Toubhans, et al., J. Appl. Phys. 63, 892-899 (1988).

17. G. M. Davis, M. C. Gower, F. O'Neill, and I. C. E. Turcu, Appl. Phys. Lett. 53, 1583-1585 (1988)

18. M. Chaker, B. L. Fontaine, C. Y. Côté, J. C. Kieffer, H. Pépin, et al., J. Vac. Sci. Technol. B 10, 3239-3242 (1992).

19. M. Chaker, J. F. Pelletier, and J. C. Kieffer, in Materials Aspects of X-Ray Lithography, G. K. Celler and J. R. Maldonado, Eds. (Materials Research Society, San Francisco, CA, USA, 1993) pp. 151-167.

20. R. Kodama, T. Mochizuki, K. A. Tanaka, and C. Yamanaka, Appl. Phys. Lett. 50, 720-722 (1987).

21. C. B. Dane, L. E. Zapata, W. A. Neuman, M. A. Norton, and L. A. Hackel, IEEE J. Quantum Electron. 31, 148-163 (1995).

22. C. B. Dane, W. A. Neuman, and L. A. Hackel, Opt. Lett. 17, 1271-1273 (1992).

23. Princeton Instruments, Inc., 3660 Quakerbridge Rd., Trenton, NJ, 08619 USA.

24. B. L. Henke, P. Lee, T. J. Tanaka, R. L. Shimabukuro, and B. K. Fujikawa, At. Data Nucl. Data Tables 27, 1-144 (1982).

25. D. R. Kania, L. Pan, H. Korblum, P. Bell, et al., Rev. Sci. Instrum. 61, 2765-2767 (1990).

26. D. R. Kania, H. Kornblum, B. A. Hammel, J. Seely, C. Brown, et al., Phys. Rev. A 46, 7853-7868 (1992).

27. D. R. Kania, L. S. Pan, P. Bell, O. L. Landen, H. Kornblum, et al., J. Appl. Phys. 68, 124-130 (1990). 


\title{
ThreE-Dimensional Simulations of ABLATIVE HYDRODYNAMIC INSTABILITIES IN INDIRECTLY DRIVEN TARGETS
}

\author{
M. M. Marinak \\ S. W. Haan \\ R. E. Tipton \\ S. V. Weber \\ B. A. Remington
}

\section{Introduction}

To model ignition in a National Ignition Facility (NIF) capsule implosion, we must understand the behavior of instabilities that can cause breakup of the pellet shell. During a capsule implosion, shocks that transit the shell cause growth of perturbations at the surface or at an interface because of a Richtmyer-Meshkov type of instability. ${ }^{1}$ Following shock breakout, or earlier for a shaped pulse, the low-density ablated plasma accelerates the pusher, and the ablation front is Rayleigh-Taylor (RT) unstable. ${ }^{2}$ Ablation and finite density gradients have the effect of stabilizing the short wavelength modes. Unstable modes present on the outer surface grow and feed through to the inner surface. Once the shell encounters the rebounding shock from the capsule center, it decelerates and the inner surface becomes RT unstable. If perturbations grow large enough, pusher material mixes into the core, degrading implosion performance.

Capsule designs for the NIF depend on ablative stabilization and saturation to prevent perturbations initially present on the capsule surface from growing large enough to quench ignition. The shape of the perturbation is a key factor in determining the amplitude at which its RT growth saturates. Classical RT experiments of an air-water interface and third-order theory carried out by Jacobs and Catton ${ }^{3}$ demonstrated that for a particular rectilinear wave number $k=\left(k_{x}^{2}+k_{y}^{2}\right)^{1 / 2}$, the symmetric square mode $\left(k_{x}=k_{y}\right)$ will start to saturate at the largest amplitude of any mode. Two- and threedimensional (2- and 3-D) simulations by Dahlburg et al. ${ }^{4}$ revealed the same dependencies in thin foils. Since the 3-D shape affects the perturbation saturation and ultimately capsule performance, a quantitative understanding of 3-D shape effects on perturbation growth is of considerable interest to inertial confinement fusion (ICF) research.

In this article, we examine the first simulations and experiments to study the effect of 3-D perturbation shape on instability growth and saturation in indirectly driven targets. The first section discusses HYDRA, the radiation hydrodynamics code we developed for these simulations. The subsequent section examines 3-D shape effects in single-mode perturbations in planar foil simulations and experiments. A discussion of the evolution of multimode perturbations on planar foils is followed by a discussion of 3-D simulations of instability growth in Nova capsule implosions.

\section{HYDRA}

We developed a new 3-D radiation hydrodynamics code, called HYDRA, to perform these simulations. HYDRA uses a structured mesh and has arbitrary Lagrange Eulerian (ALE) capability. Several forms of equations of state (EOS) are available, including the EOS4 tabular database and the inline quotidian EOS (QEOS). Multigroup radiation diffusion is implemented using tabular opacities. The thermal conduction routine applies conductivities calculated with the model of Lee and More. ${ }^{5}$ HYDRA has an Eulerian interface tracker that allows users to resolve material interfaces while making full use of its ALE capability. "Mixed" zones, which contain one or more material interfaces, are subdivided into separate components. Radiation transport, thermal conduction, and other physical processes are treated separately for all of the components of the mixed zones. The interface tracker allows one to avoid distorted meshes, and the accuracy problems they can cause, even while resolving shear flows. 
The capability of coupling several adjacent zones together has been incorporated in HYDRA. This allows greater freedom in zoning without the full additional computational overhead and increased memory requirements associated with an unstructured mesh. This coupling is essential to avoid the small Courant time step limits that would occur, for example, in narrow zones near the center or near the pole of a 3-D capsule implosion simulation. This capability can also be applied in the presence of mixed zones, allowing one to adjust the local resolution in different directions relative to a material interface.

We developed a technique for treating radiation transport that enables us to run 3-D simulations of a variety of targets with viable computational requirements. The method involves two separate calculations. In the first, we obtain a detailed treatment of the spectrum by using a large number of photon groups while the geometry is approximated. All the detailed spectrum and opacity information is then collapsed into group constants for a small number of photon groups. In the second calculation, these few group opacities are applied in a simulation that treats the detailed spatial variation with use of the diffusion operator. The method yields estimates of the group opacities that are vastly superior to those obtained with the standard Rosseland mean. Compared with the standard method, this new development has enabled us to perform highly accurate numerical solutions at a small fraction of the computational expense. The implementation of this method, combined with the high run speeds and reduced memory requirements attainable with algorithms written on a structured mesh, make HYDRA well suited for a variety of problems that contain some degree of symmetry.

\section{Shape Effects}

\section{Single-Mode Perturbations on Planar Foils}

We first examine foils having single-mode rectilinear perturbations $\psi=a \cos \left(k_{x} x\right) \cos \left(k_{y} y\right)$, with $k_{x}=k_{y^{\prime}} k_{x}=3 k_{y^{\prime}}$ and $k_{y}=0$ (2-D). Each perturbation has the same wave number $k=\left(k_{x}^{2}+k_{y}^{2}\right)^{1 / 2}=2 \pi / 80 \mu \mathrm{m}^{-1}$. To study growth extending from the linear regime through the late nonlinear stage, we use a hypothetical drive that gives a long period of acceleration and thus large growth factors. We take the drive spectrum from a LASNEX hohlraum simulation. The drive temperature history imposed starts with a foot at $\sim 100 \mathrm{eV}$, then ramps up to $162 \mathrm{eV}$ over the first $1 \mathrm{~ns}$ and is then held constant through $4 \mathrm{~ns}$. This drive is used for all the planar simulations discussed here except for simulations of Nova experiments. Supertransition array (STA) opacities for $\mathrm{CH}(\mathrm{Br})$ are used. The foil is initially $50 \mu \mathrm{m}$ thick. We use an ideal-gas EOS in this simulation for generality.

Figure 1 shows the evolution of the fundamental mode amplitude of areal density for the three perturbation aspect ratios listed above. Since each perturbation has the same wave number and is initialized with the same amplitude, each should grow at the same rate during the shock transit and early acceleration phases when the perturbation is linear; Fig. 1 shows that this is the case. The two shocks generated during the foot and subsequent rise to the peak of the drive coalesce as they break out of the foil at $1 \mathrm{~ns}$. After a period of linear RT growth, the point at which each mode begins to saturate shows a clear progression with the mode shape. As the mode shape is varied from the least symmetric 2-D mode to the most symmetric 3-D square mode, the (a)

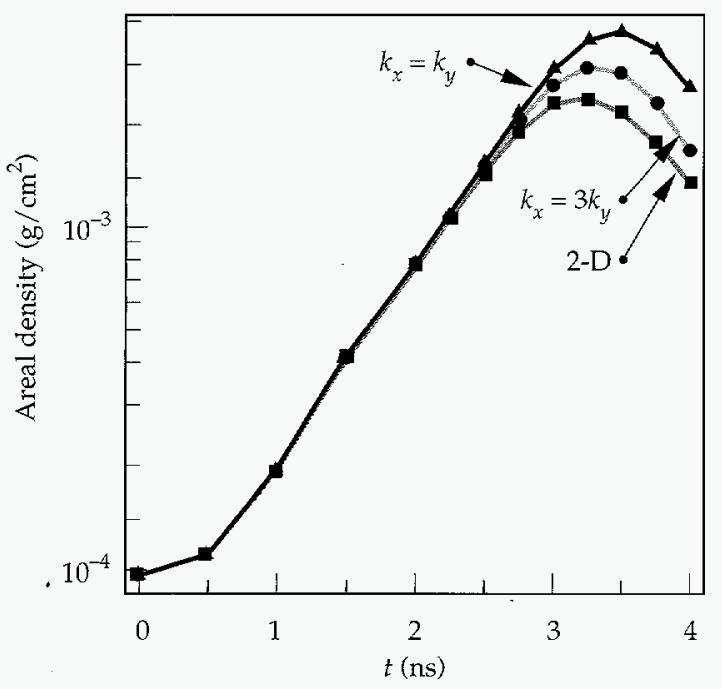

(b)

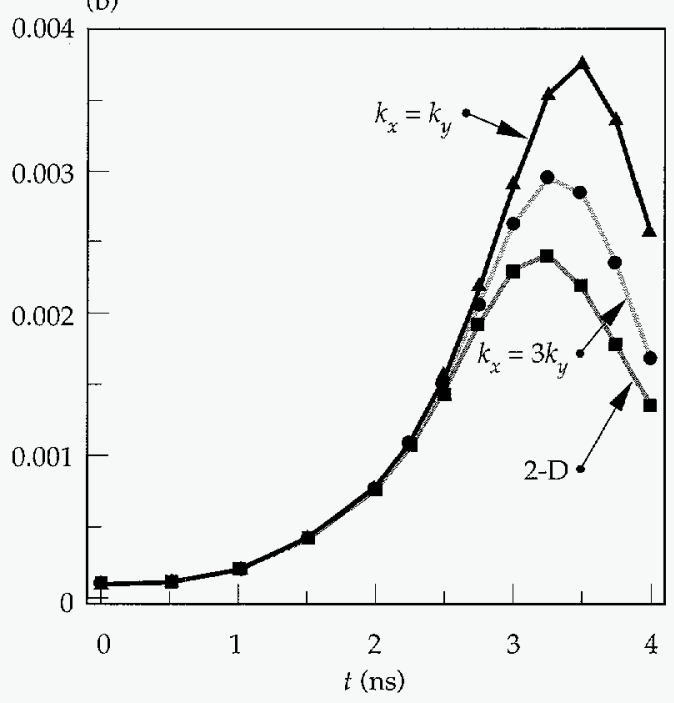

FIGURE 1. Evolution of the fundamental Fourier mode of areal density for three Cartesian mode simulations. Mode amplitudes are plotted (a) on a logarithmic scale and (b) on a linear scale; symbols indicate calculated points. (50-05-0595-1109pb01) 
perturbation begins to saturate later and achieves a larger amplitude. The 3-D square mode reaches a peak amplitude $56 \%$ larger than the 2-D mode.

Figure 2 shows the $\rho=0.3 \mathrm{~g} / \mathrm{cm}^{3}$ isodensity surface at 3.25 ns for the 3-D square and 2-D modes. In the nonlinear phase, the 3-D square mode has evolved into a broad bubble surrounded by narrow spikes and adjoining spike sheets. The peak-to-valley $(\mathrm{p}-\mathrm{V})$ displacements of the isodensity contours are very similar between the 2- and 3-D modes. The larger amplitude of the square mode is manifest primarily because of its greater density in the spike.

The hexagonal mode perturbation,

$\psi=a\left[\cos \left(\frac{k_{0} x}{2}\right) \cos \left(\frac{\sqrt{3} k_{0} y}{2}\right)+\cos \left(k_{0} x\right)\right]$ consists of a hexagonally packed array of either bumps or pits, depending on the sign of the perturbation. Reversing the sign of this mode results in a different shape, not just a shift in the phase. Simulations of foils with the two orientations of hexagonal-mode perturbations show rather different evolution in the nonlinear regime. The bubble-centered orientation, consisting of an array of pits, develops into hexagonal bubbles surrounded by spike sheets in the nonlinear stage as shown in Fig. 3(a). The spike-centered orientation, shown in Fig. 3(b), develops isolated individual spikes. The maximum $\mathrm{p}-\mathrm{V}$ areal density modulations obtained are $45.3 \mathrm{~g} \mathrm{\mu m} / \mathrm{cm}^{3}$ for the bubble-centered perturbation and $83.8 \mathrm{~g} \mathrm{\mu m} / \mathrm{cm}^{3}$ for the spike-centered perturbation. Growth of the spike-centered mode is favored on the ablating foil because it has a smaller surface area subject to ablation and because more material surrounds the base of the axisymmetric spike. The bubble-centered
FIGURE 2. Isodensity plots for $\rho=0.3 \mathrm{~g} / \mathrm{cm}^{3}$ at $3.25 \mathrm{~ns}$ : (a) for the simulations of the $k_{x}=k_{y}$ square mode and (b) for the $k_{y}=0$ (2-D) mode. One period of the perturbations is shown $(k=2 \pi / 80$ $\mu \mathrm{m}^{-1}$ ). For each plot, the perspective is from the side of the target ablated by $x$ rays. (50-05-0595-1110pb01)

FIGURE 3. Plots of areal density for the two hexagonal-mode orientations in the saturated regime. (a) Bubble-centered perturbations; (b) spikecentered perturbations. Brightest regions correspond to spikes. (50-05-0595-1167pb02)
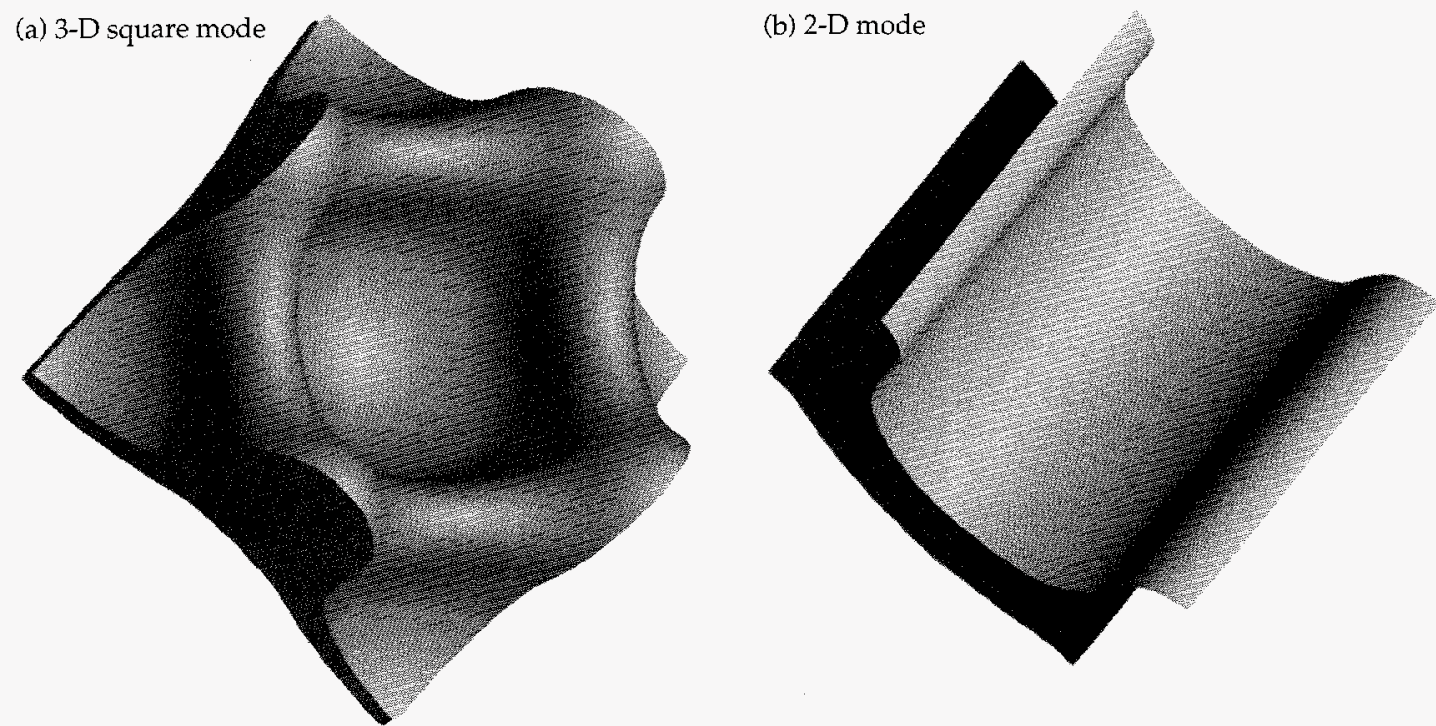

(a) Bubble-centered perturbations

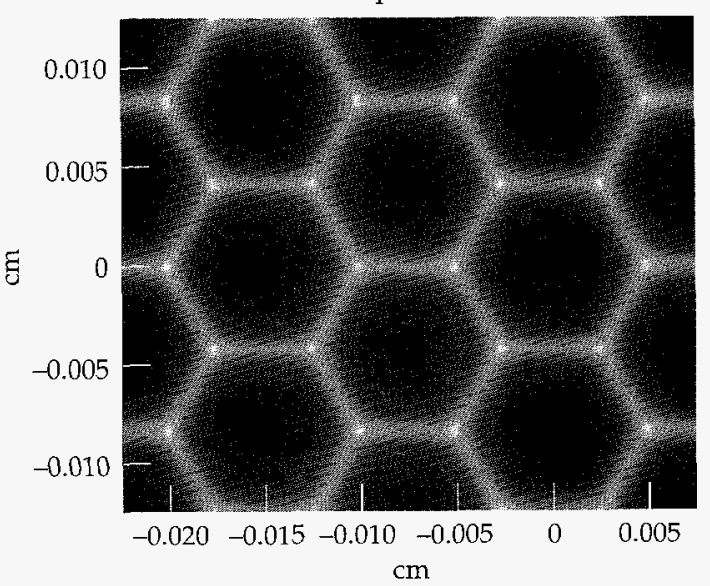

(b) Spike-centered perturbations

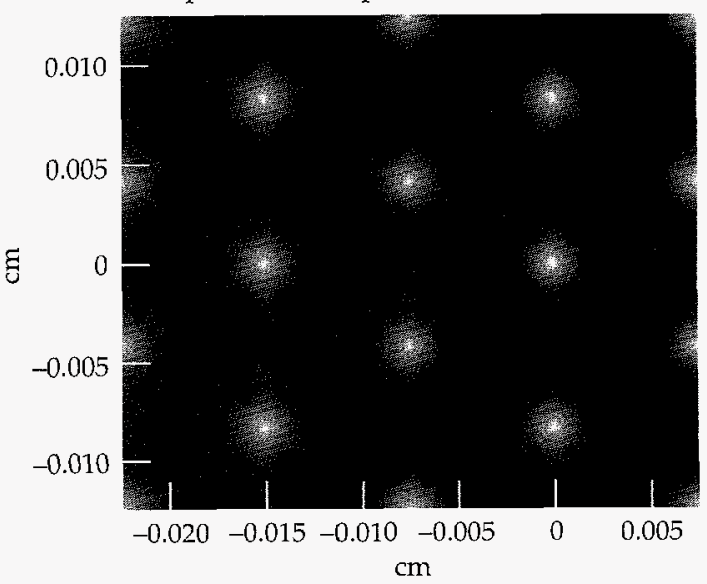


perturbation attains smaller minimum areal density, and it burns through the foil faster. The axisymmetric bubble is the perturbation shape most dangerous to the survival of thin capsule shells.

\section{3-D Shape Effects in Experiments}

We performed an experimental study of single-mode shape effects with planar foils on Nova. In this article, we consider some of these results and compare them with simulations. For these experiments, surface perturbations are molded onto one side of a $\mathrm{CH}(\mathrm{Br})$ foil $\left(\mathrm{C}_{50} \mathrm{H}_{47} \mathrm{Br}_{2.7}\right) 750 \mu \mathrm{m}$ in diameter, 53-57 $\mu \mathrm{m}$ thick, density $1.26 \mathrm{~g} / \mathrm{cm}^{3}$. The foil is mounted across a hole in

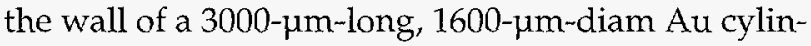
drical hohlraum with the perturbations facing inward. It is illuminated from the back by a 500-700- $\mathrm{mm}$-diam source of "backlighter" $x$ rays created by irradiating a disk of either Rh or Sc with one or two Nova beams. We use measurements of the transmitted intensity of backlighter $x$ rays to determine the growth in the areal density of the perturbations. For targets with 2-D perturbations, a time-resolved image $I(x, t)$ is recorded onto film using a $22 \times$ magnification streaked Wölter $x$-ray microscope ${ }^{6}$ with a Rh backlighter. For foils with 3-D perturbations, we collect gated images $I(x, y, t)$ at several times during the shot using the flexible $x$-ray pinhole imager $(\mathrm{FXI})^{7}$ in conjunction with a Sc backlighter. We characterized the spatial resolution of these instruments and included it wherever simulations are compared with data.

Eight $0.351-\mu \mathrm{m}, 2.2-\mathrm{kJ}$ shaped Nova beams irradiate the hohlraum. The shaped drive, termed laser pulse shape 23 , has a low-intensity $\sim 1.5$-ns foot, which is followed by a rapid increase to peak power at $2.5-3.0 \mathrm{~ns}$ with a peak-to-foot intensity ratio of 10 . Our most recent model of this $x$-ray drive is based on a 2-D LASNEX $\mathrm{Au}$ hohlraum simulation. During the foot of the pulse $(t<2 \mathrm{~ns})$, we increase the preheat portion of the spectrum $(h v>1.4 \mathrm{keV}$ ) by a factor of 10 over that predicted by LASNEX while maintaining the same total power in the drive. The result is that during the foot an average of $8 \%$ of the total energy in the drive spectrum is above $2 \mathrm{keV}$. This adjustment stems from uncertainty in the total preheat energy deposited in the foil early in time. It has the effect of reducing growth somewhat, systematically improving the fit throughout the database. This drive model reproduces well the foil trajectories and thus the zero-order hydrodynamics. ${ }^{8}$

We examine the effect of perturbation shape on saturation amplitude by comparing a foil having a square-mode perturbation $\left(k_{x}=k_{y}\right)$ with one having a 2-D perturbation $\left(k_{y}=0\right)$, each having the same effective wave number $k=2 \pi / 50 \mathrm{\mu m}^{-1}$ and similar amplitudes. We used a laser ablation technique to create the periodic 3-D perturbation on the mold. ${ }^{9}$ With the relatively short acceleration period of pulse shape 23, large initial amplitudes are required to allow the foils to evolve well into the nonlinear regime. Fourier analysis of an $x-$ ray radiograph of the square-mode foil yields the initial amplitude of the fundamental perturbation as $2.7 \mu \mathrm{m}$. Small-amplitude higher harmonics are also present, but their amplitudes are typically less than $10 \%$ of the fundamental mode amplitude. The foil with the 2-D perturbation had an amplitude of $2.5 \mu \mathrm{m}$. In the HYDRA simulation of the square-mode foil, the surface perturbation initialized includes the predominant $\cos \left(k_{x} x\right)$ $\cos \left(k_{y} y\right)$ modes as well as $\cos \left(m k_{x} x\right) \cos \left(n k_{y} y\right)$ modes having $(m, n)=(0-3,0-3)$ taken from the radiograph.

Figure $4(a, b)$ compares images of $\ln$ (exposure) calculated from the simulation and measured with the FXI at $4.3 \mathrm{~ns}$, including the effect of instrumental resolution. At this time, the mode has progressed well into the nonlinear phase. The HYDRA simulation reproduces well the structure of the dark narrow spikes, adjoining spike sheets, and wide bubbles. A slight elongation of the central spike is due to the small asymmetry present in the initial perturbation, contained in higher harmonic modes. Figure $4(c, d)$ makes the same comparison as surface plots of $\ln ($ exposure) vs $(x, y)$, where the bubble is centered instead of the spike.

Figure 5(a) compares the measured time evolution of the fundamental modes for the 2- and 3-D foils with results from simulations. The data represented by the solid squares and open circles correspond to the $\cos \left(k_{x} x\right) \cos \left(k_{y} y\right)$ components of the Fourier transform of $\ln$ (exposure) for the $k_{x}=k_{y}$ perturbation. The open diamond symbols represent the amplitude of the $\cos \left(k_{x} x\right)$ 2-D perturbation. Curves from the HYDRA simulations match well the time evolution of the data. The 3-D mode saturates later at a larger amplitude, consistent with our expectations. Figure 5(b) compares higher harmonics of measured growth factors in Fourier amplitude of $\ln$ (exposure) with values from the simulation of the square mode. Observe that mode $(0,2)$ reverses sign at $3.8 \mathrm{~ns}$ as a consequence of mode coupling. Despite initially having different signs, the amplitudes of modes $(0,2)$ and $(2,0)$ tend to converge later in the nonlinear regime as the perturbation evolves toward a more symmetric shape. This dynamic evolution shows that the growth of the most symmetric 3 -D shape is favored in the saturated regime.

Our results are consistent with the notion that the saturation amplitudes are a consequence of the mode shape. The calculated flow carries material away from the more spherical 3-D bubble on all sides, allowing it to transit more easily into the spike regions. The resulting faster growth for the 3-D bubble-spike pattern increases the rate of mass flow into the spikes relative to the rate at which it is consumed by ablation, allowing the 3-D square mode to achieve a larger peak amplitude. 
FIGURE 4. (a) Contour plot of $\ln$ (exposure) from the simulation at $4.3 \mathrm{~ns}$ for one period on the $k_{x}=k_{y}$ foil. Dark regions have low exposures, corresponding to high areal density. The effect of the instrument resolution function has been included. The FXI image (b) has been averaged over an area encompassing $4 \times 4$ periods on the foil. (c), (d) The same numerical and experimental results are displayed in the form of 3-D surface plots in which the height is proportional to intensity. (50-05-0595-1116pb01) (a) HYDRA simulation

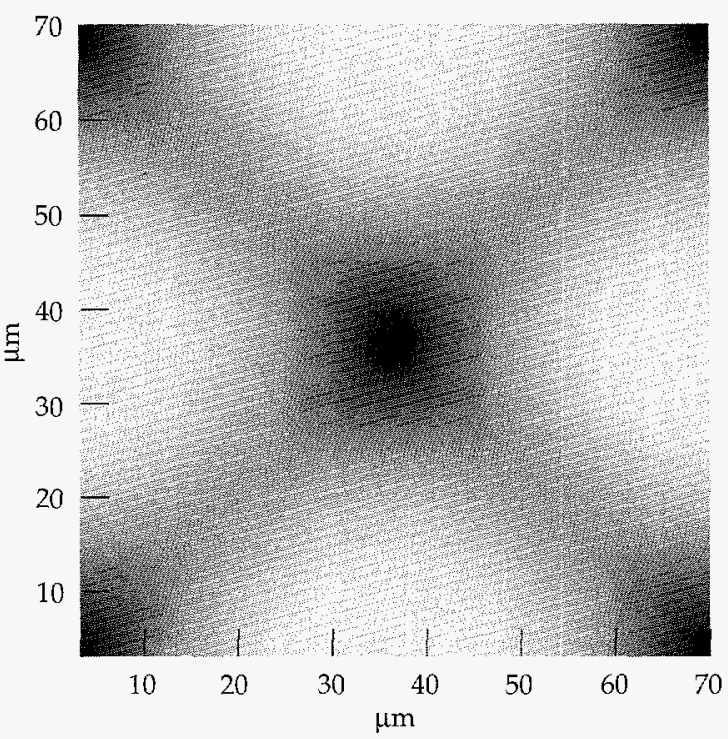

(c) 3-D surface plot of (a)

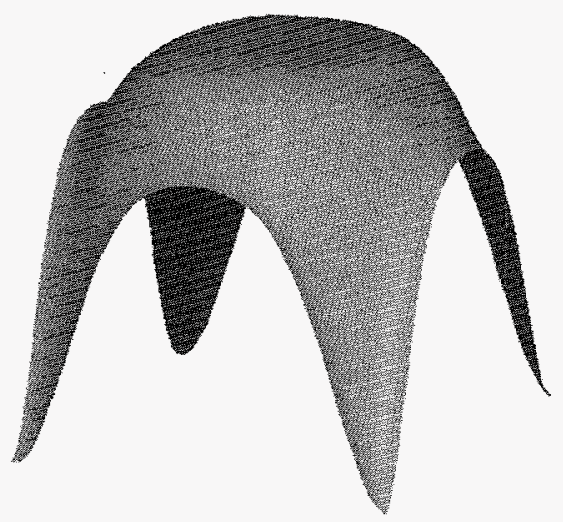

(b) FXI image

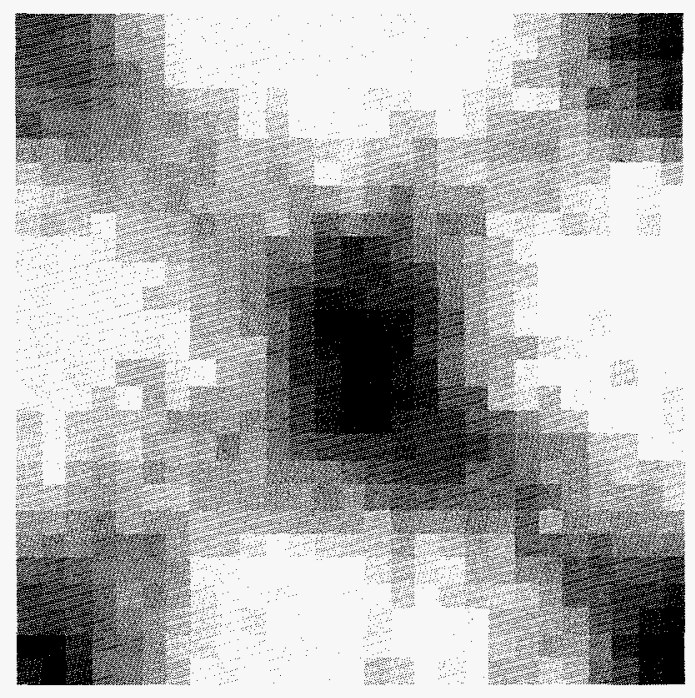

(d) 3-D surface plot of (b)

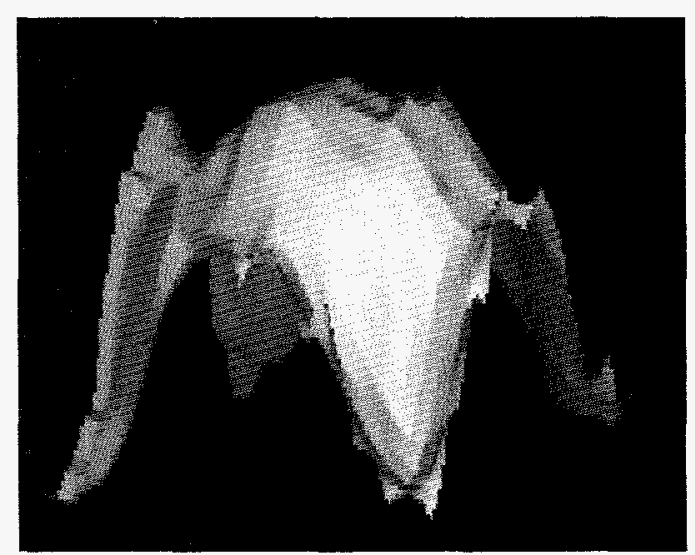

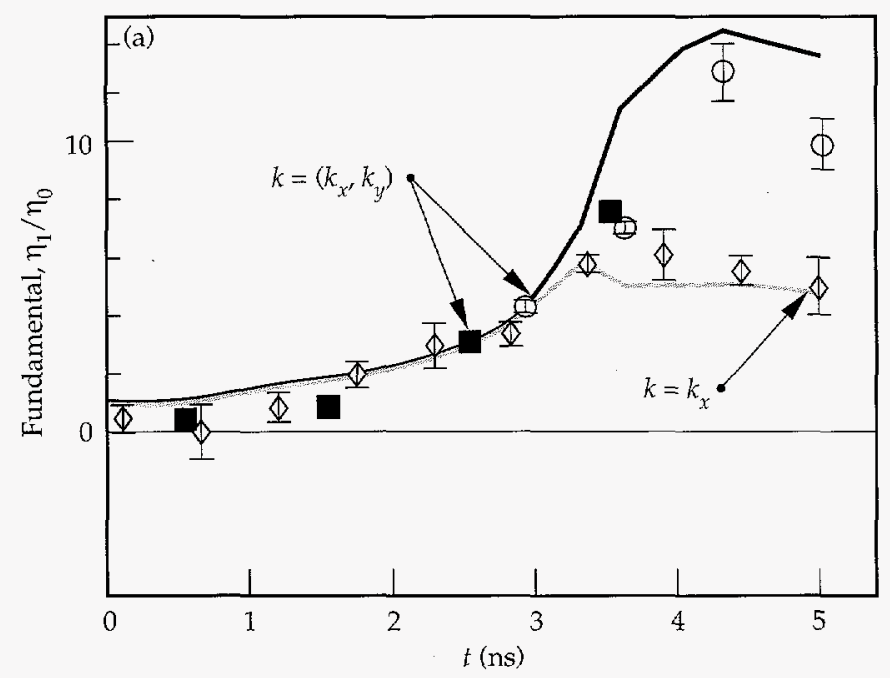

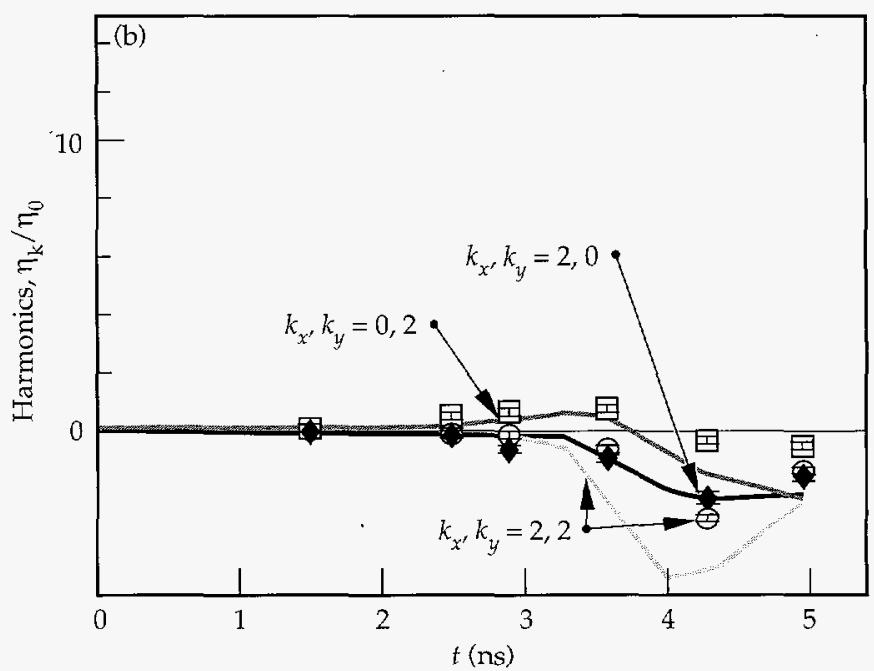

FIGURE 5. Measured and simulated perturbation Fourier amplitudes vs time, normalized to the fundamental mode amplitude at $t=0$.

(a) The solid squares and open circles represent the measured growth factor of $\ln$ (exposure) of the fundamental $k_{x}=k_{y}$ mode. The open diamonds are the same quantity for the 2-D mode. The curved lines are the corresponding results from HYDRA simulations. (b) The open circles, solid diamonds, and open squares correspond to the square-mode higher harmonics $(2,2),(2,0)$, and $(0,2)$, respectively. The curved lines give the corresponding simulation results. Experimental error bars represent the corresponding magnitude of the background level in Fourier space. $\quad(20-03-0395-0791 \mathrm{pb02})$ 


\section{Multimode Perturbations on Planar Foils}

Capsule implosion experiments currently being performed on Nova examine the growth of surface perturbations created by ablation with an excimer laser. ${ }^{9}$ Each individual perturbation formed by the laser is in the shape of a pit. Many of these pits may be placed randomly on the capsule to create a spectrum that is random for the low-l mode numbers, but the higher modes that form the shapes of the individual pits are not randomly phased. There is some difference between the nonlinear evolution of this perturbation and that for a truly random spectrum of modes.

A planar surface that contains a number of Gaussian perturbations centered at $\left(x_{i}, y_{i}\right)$ with amplitudes $a_{i}$ each having identical widths $\sigma$ is represented as

$Z(x, y)=\sum_{i} a_{i} \exp \left\{\frac{-\left[\left(x-x_{i}\right)^{2}+\left(y-y_{i}\right)^{2}\right]}{\sigma^{2}}\right\}$.

We consider a system with reflection symmetry across each boundary. By using the convolution theorem, we obtain the Fourier spectrum:

$Z\left(k_{x}, k_{y}\right)=16 \pi \frac{\sigma^{2}}{L^{2}} \sum_{i} a_{i} \exp \left[\frac{-\left(k_{x}^{2}+k_{y}^{2}\right) \sigma^{2}}{4}\right]$
$\times \cos \left(k_{x} x_{i}\right) \cos \left(k_{y} y_{i}\right)$,

where $L$ is the foil width. For $k_{x}$ or $k_{y}=0$, the amplitude of the mode coefficient $a_{i}$ is multiplied by 0.5 when we express the spectrum in terms of cosine modes instead of complex exponentials.

Consider two similar foils. In the first, the initial perturbation consists of a set of Gaussian bumps (all $a_{i}$ positive). Figure 6(a) shows a contour plot of the initial areal density. In the second, the signs of all of the $a_{i}$ are reversed, which is the corresponding pattern formed with Gaussian pits. [A contour plot for this case would be just the reverse of that shown in Fig. 6(a).] Figure 6(b) is a contour plot of the areal density at $5.0 \mathrm{~ns}$, in the saturated regime, for the first case (Gaussian bump perturbations). The individual bumps have evolved into spike sheets surrounding bubbles. Figure 6(c) shows the nonlinear stage for the second case (Gaussian pit perturbations) at $4.5 \mathrm{~ns}$, which also develops broad bubbles surrounded by narrow spike sheets in the nonlinear regime. This bubble-spike structure is characteristic of the nonlinear development of a random multimode foil. ${ }^{10}$ In the saturated regime, both foils have very similar values for $\mathrm{p}-\mathrm{V}$ and $\mathrm{rms}$ areal density. The ratio of the spike amplitude to bubble amplitude is usually taken as $1+A$, where $A$ is the Atwood number, which is $\sim 1$ here. We define the bubble side areal density as the rms average over all locations at which the areal density is less than the average value. For the foil with the bump perturbations, the ratio of spike side to bubble side areal density is 2 in the saturated regime, a value that is typical for random multimode perturbations as well. But the bubble-centered random Gaussian foil has an rms areal density on the bubble side essentially equal to that on the spike side in the saturated regime. This bubble amplitude is unusually large. Because of its larger bubble growth, the foil with bubble perturbations burns through first, prior to $5.0 \mathrm{~ns}$ for this case. (a) Initial areal density (Gaussian bumps)

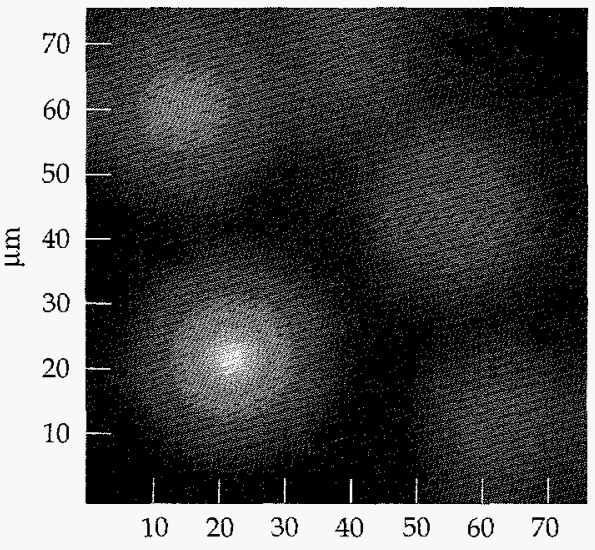

(b) Bumps at $5.0 \mathrm{~ns}$

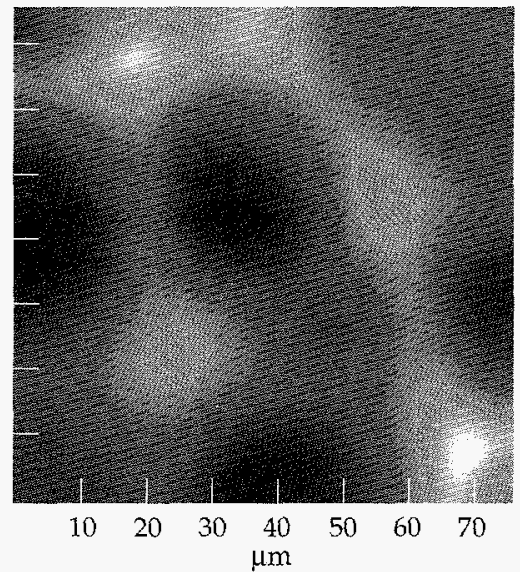

(c) Bubble case at $4.5 \mathrm{~ns}$

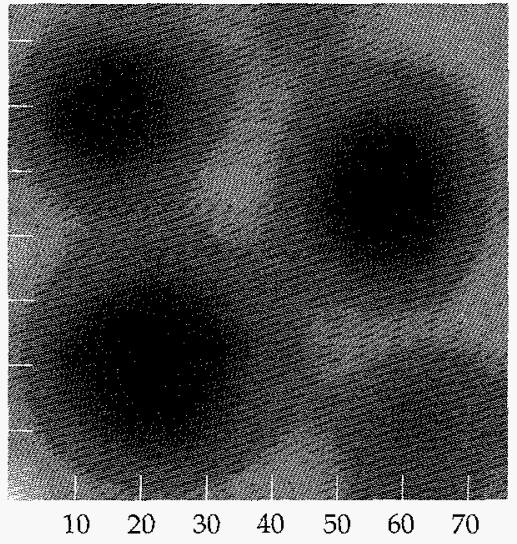

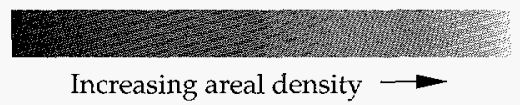

FIGURE 6. Contour plots of areal density for foils with randomly placed Gaussian perturbations. (a) Initial areal density for bumps with various amplitudes. Bumps appear as bright spots. (b) Foil with bump perturbations at $5.0 \mathrm{~ns}$ in the saturated regime. (c) Corresponding foil with bubble perturbations at $4.5 \mathrm{~ns}$. $\quad(50-05-0595-1111 \mathrm{pb} 01)$ 
The accelerated pace at which the random bubble perturbation burns through the foil is the most significant characteristic distinguishing it from a truly random perturbation.

Next we consider a random multimode foil on which the imposed perturbation

$\psi=\sum_{m=0}^{8} \sum_{n=0}^{8} a_{m n} \cos \left(m k_{0} x\right) \cos \left(n k_{0} y\right)$

consists of 80 modes with random amplitudes, yielding an initial rms perturbation amplitude of $0.61 \mu \mathrm{m}$ rms. With a fundamental wavelength of $250 \mu \mathrm{m}$, the spectrum of perturbations spans the range of wavelengths most relevant to indirectly driven targets. The foil thickness is initially $50 \mu \mathrm{m}$.

Figure 7(a) shows a contour plot of the initial multimode-foil areal density. The simulation shows that by $2.5 \mathrm{~ns}$ [Fig. 7(b)], in the early nonlinear stages of the foil evolution, mode coupling has caused broadening of the bubbles and the formation of narrow spike sheets. Areal density contours calculated with the Haan second-order mode-coupling theory ${ }^{11}$ are in good agreement with those from the simulation in the early nonlinear stage. At this time the perturbations still bear some resemblance to those initially on the foil. By $4.0 \mathrm{~ns}$ [Fig. 7(c)], strongly nonlinear interactions lead to increased average width of the features. This process is termed bubble merger in physical space or inverse cascade in Fourier space.

Layzer ${ }^{12}$ derived the terminal-rise velocity of a bubble in a semi-infinite incompressible fluid: $u_{b}=a(g \lambda)^{1 / 2}$.

The Froude number $a$ is 0.23 for a 2-D bubble and 0.36 for a round 3-D bubble. Larger bubbles have less kinematic drag per unit volume, so bubble expansion in the nonlinear regime leads to increasing bubble rise velocities. When driven far enough into the nonlinear regime, substantial displacements develop between bubbles in the longitudinal direction due to the spread in their rise velocities. Some displacement is already apparent in Fig. 8(a), which shows the isodensity (a) Initial areal density

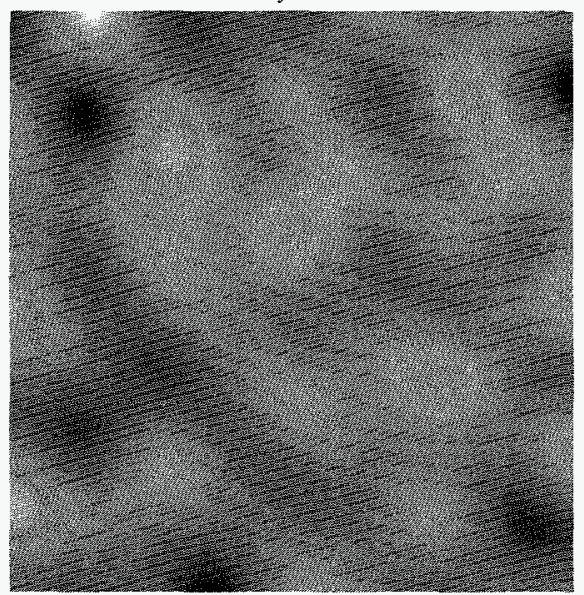

(b) $2.5 \mathrm{~ns}$ areal density

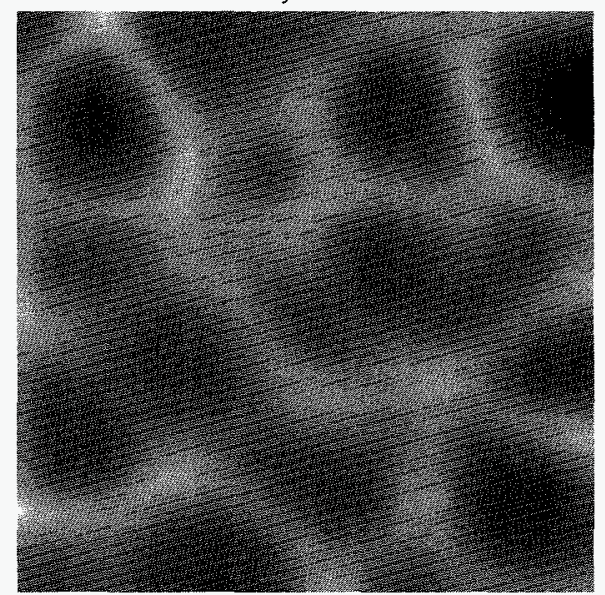

(c) $4.0 \mathrm{~ns}$ areal density

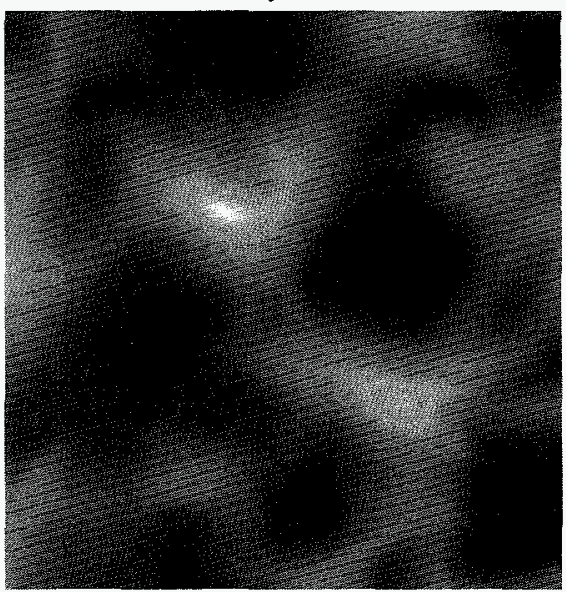

FIGURE 7. Areal density for random multimode foil (a) initially, (b) at $2.5 \mathrm{~ns}$, and (c) at $4.0 \mathrm{ns.} \mathrm{Bumps} \mathrm{appear} \mathrm{as} \mathrm{bright} \mathrm{spots.} \mathrm{(50-05-0595-1112pb01)}$

FIGURE 8. (a) Threedimensional plot of $0.75 \mathrm{~g} / \mathrm{cm}^{3}$ density surface at $2.5 \mathrm{~ns}$. View is of the side of the foil ablated by $x$ rays. (b) The $0.3 \mathrm{~g} / \mathrm{cm}^{3}$ density surface at $3.0 \mathrm{~ns}$. View is of bubbles on the side of the foil away from the $x$-ray source. (50-05-0595-1113pb01) (a) $2.5 \mathrm{~ns}$ isodensity surfaces

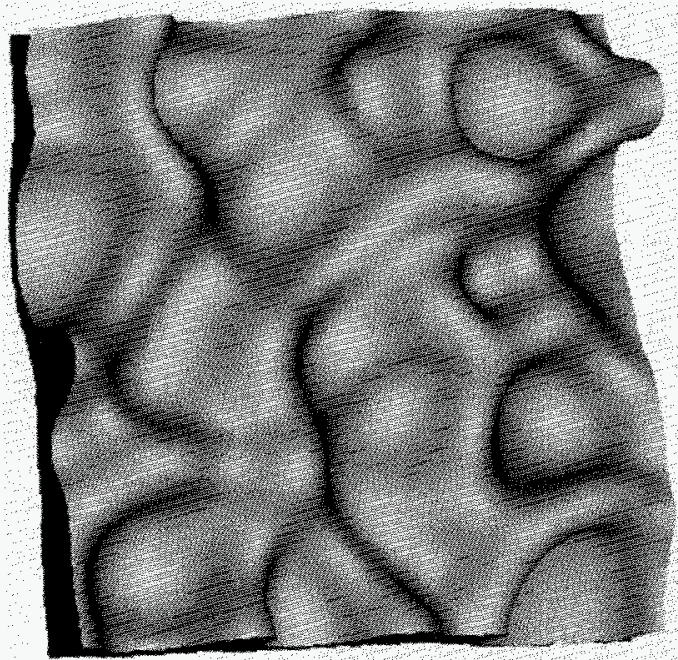

(b) $3.0 \mathrm{~ns}$ isodensity surfaces

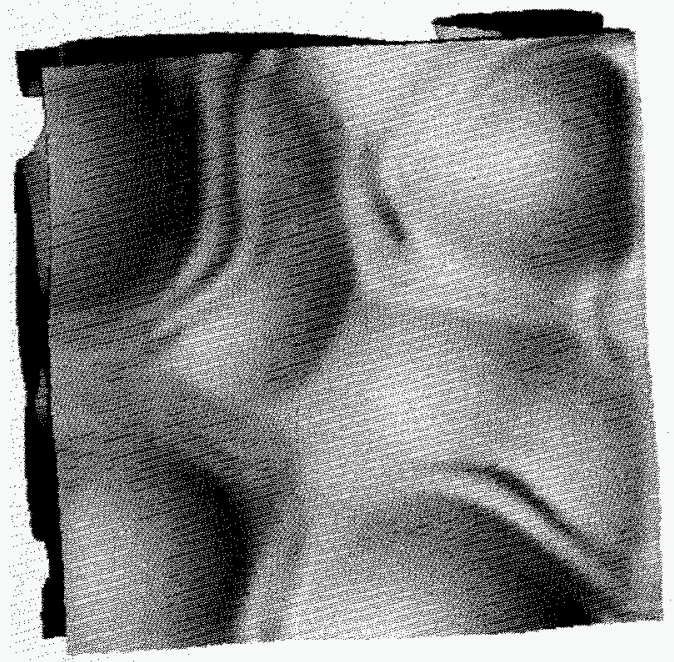


surface $\rho=0.75 \mathrm{~g} / \mathrm{cm}^{3}$ at $2.5 \mathrm{~ns}$, early in the nonlinear stage. As this longitudinal displacement increases, the bubbles tend to expand laterally to fill the available space. As they move ahead of the others, the tips of the largest bubbles become round, minimizing drag and yielding the highest rise velocity. The isodensity surface shown in Fig. 8(b) shows that the largest bubbles have advanced farthest toward the back side of the foil at $3.0 \mathrm{~ns}$. These bubbles are already much broader than the features of the initial foil perturbation.

Figure 9 plots the Fourier spectrum at $0,2.5$, and $4.0 \mathrm{~ns}$. By $2.5 \mathrm{~ns}$, the spectrum peaks at the wave number having the highest linear growth rate. Mode coupling has driven up higher harmonics. By $4.0 \mathrm{~ns}$, the inverse cascade is apparent as the peak of the spectrum shifts to lower wave numbers. The spectrum has the appearance of a turbulent form, with mode coupling transferring spectral power that originates from the growth of moderate wavelengths to shorter, ablatively stabilized wavelengths and to very long wavelengths. There is little change in the ablatively stabilized portion of the spectrum (above $5000 \mathrm{~cm}^{-1}$ ) during the saturated phase of evolution, after $3.0 \mathrm{~ns}$.

We used the Haan saturation model to predict rms amplitudes of bubbles and spikes in the weakly nonlinear regime arising from isotropic random multimode perturbations. ${ }^{13}$ In the model, individual modes grow exponentially in the linear regime until the modal

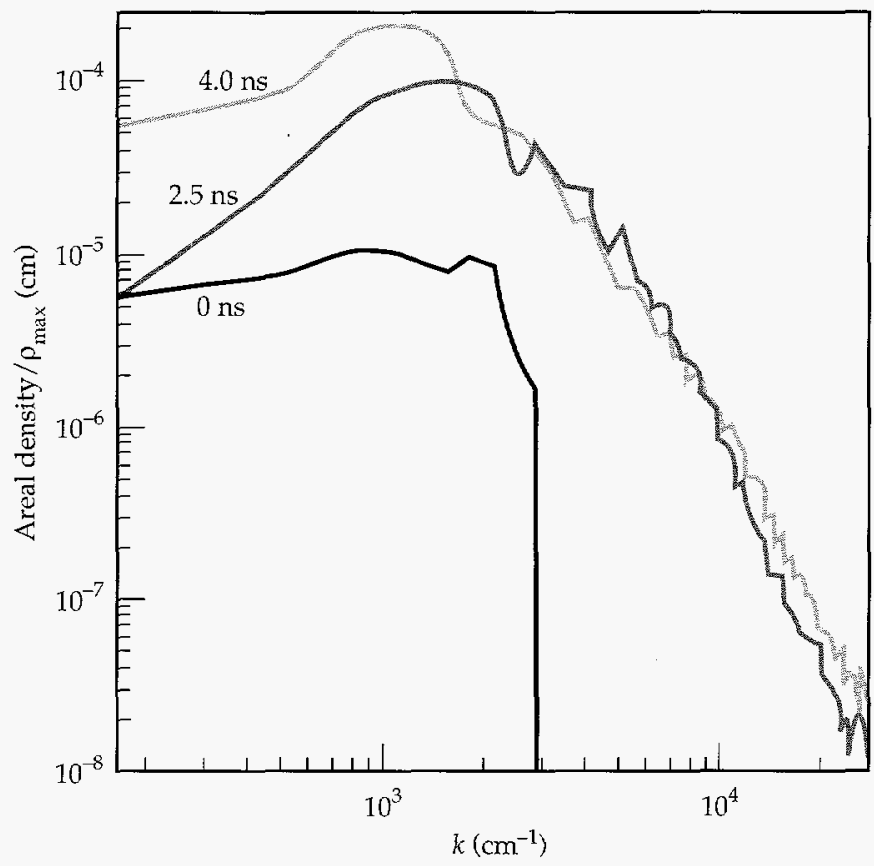

FIGURE 9. Fourier spectra of mode amplitudes vs mode number for multimode foil at $0,2.5$, and $4.0 \mathrm{~ns}$. (50-05-0595-1114pb01) saturation amplitude $v / L k^{2}$ is attained. Then the mode growth changes to secular. Rms bubble and spike amplitudes are obtained by summing modal amplitudes in quadrature. A value of $v=2$ is generally used for the saturation parameter. As shown in Fig. 10, the departure of the rms bubble growth from linear theory in the simulation initially resembles the model predictions for $v=1$. The simulated bubble areal density soon rolls over because of burn-through of the foil, which is not accounted for in the saturation model. Since the foil is initially only $50 \mu \mathrm{m}$ thick, thin-foil effects appear to have an influence on the saturation amplitude.

Perturbation growth continues in low-density regions, and thus contributes little to perturbed areal density. Despite thin-foil effects, the onset of saturation is still roughly consistent with the prediction of the Haan saturation model. This increases our confidence that the model can be used to predict rms perturbation amplitudes in the weakly nonlinear regime.

\section{Capsule Implosion Simulations}

The experimental program on Nova is beginning to examine instability growth on capsules that have pits ablated at the 92 positions corresponding to the vertices and face centers of the pattern on a soccer ball. ${ }^{9}$ The shape of an individual pit has been characterized experimentally as a superGaussian function

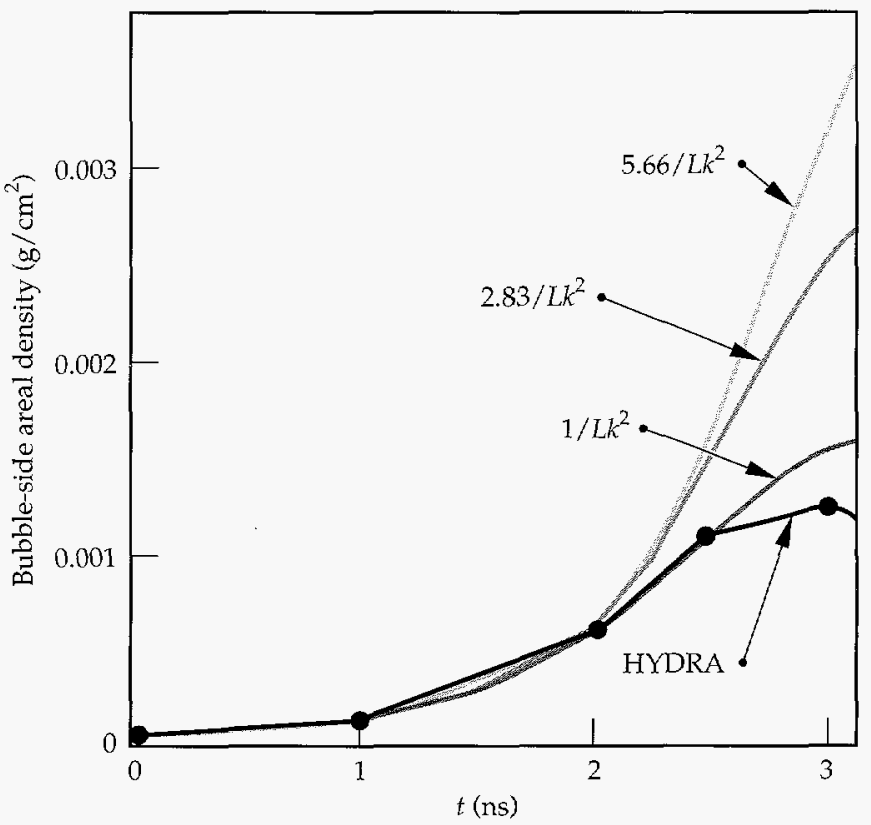

FIGURE 10. The symbols on the solid black line represent the bubble-side rms areal density for the multimode foil calculated with HYDRA. The gray-tone curves represent the bubble-side areal density predicted by the Haan saturation model for three values of the saturation parameter. After $2.5 \mathrm{~ns}$, the values from the simulation begin to roll over because of the effect of finite foil thickness, which is not accounted for in the saturation model. (50-05-0595-1115pb01) 
$\psi(r)=-a_{0} e^{-(r / \sigma) 3}$ in the radius $r$, with $a_{0}$ the pit depth, and $\sigma=28 \mu \mathrm{m}$. The overall surface perturbation resulting from these 92 pits can be expressed in terms of the spherical harmonics $Y_{l m}$ :

$G(\theta, \phi)=\sum_{i=1}^{92} \sum_{l>1}^{\infty} a_{l} \frac{4 \pi}{2 l+1} \sum_{m=-l}^{l} Y_{l m}\left(\theta_{i}^{\prime}, \phi_{i}^{\prime}\right) Y_{l m}(\theta, \phi)$,

where $a_{l}$ is the coefficient of the Legendre polynomial $P_{l}$ in an expansion for an individual pit:

$a_{l}=\frac{2 l+1}{2} \int_{0}^{\pi} P_{l}(\cos \theta) g(\theta) \sin \theta d \theta$

Here $g(\theta)$ is the shape function for an individual pit expressed in polar coordinates. For the soccer ball pattern, most of the perturbation is contained in spherical harmonics with $l=16,18$. Capsules can also be created with one hemisphere of the pattern rotated by $36^{\circ}$ so that the perturbation is reflection-symmetric about the equator. This modification has a minor impact on the mode structure and the spacing between pits. This geometry is treated exactly by simulating $1 / 20$ of a sphere over a region extending from the pole to the equator and $36^{\circ}$ in the azimuthal angle. Symmetry boundary conditions are applied at the transverse boundaries.

The Nova capsules simulated have a fuel region $220 \mu \mathrm{m}$ in radius containing equimolar amounts of $\mathrm{H}$ and $D$. The fuel is bounded by a 3 - $\mu \mathrm{m}$-thick polystyrene

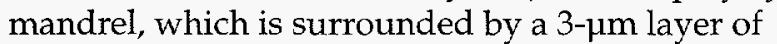
polyvinyl alcohol (PVA) and $39 \mu \mathrm{m}$ of Ge-doped polystyrene ablator. We first consider a capsule having pits $0.25 \mu \mathrm{m}$ deep on the outer surface. As the capsule shell accelerates inward, the solid angle occupied by each pit remains essentially constant while it grows into the shell. At the onset of deceleration, isolated bubbles of fuel begin to rise into the shell at the locations of the initial pits, where the fuel column density is lowest, while interconnected sheets of pusher material surrounding these bubbles fall inward.

Concurrently, the amplitude of the bubbles on the outer surface begins to decrease. Since the Atwood number at the fuel-pusher interface is $\sim 0.4$ at this time, shear leads to a Kelvin-Helmholtz instability, causing the material interface to roll up at the tips of the fuel bubbles. The characteristic "hammerhead" shapes are visible in Fig. 11, a plot of the $\rho=18 \mathrm{~g} / \mathrm{cm}^{3}$ isodensity surface at $2.1 \mathrm{~ns}$. The asymmetric shape of the roll-up, localized near the bubble tip, is due to the bubble-like shape of the initial perturbations. Fuel entrained into the rising bubbles is relatively cool, having temperatures of $\sim 400-500 \mathrm{eV}$. Thus significant neutron production arises only from the center of the hot spot in regions that are many micrometers from spike material. For this $0.25-\mu \mathrm{m}$ initial perturbation amplitude, the neutron yield is $82 \%$ of that obtained with no perturbation. Most of the neutron production has occurred before $2.1 \mathrm{~ns}$.

Simulations have been performed for a range of initial perturbation amplitudes spanning $0.25-2.5 \mu \mathrm{m}$ $p-V$. For each of these cases, the perturbation of the fuel-pusher interface becomes nonlinear quite soon after it encounters the first rebounding shock. During the first 100 ps of deceleration, the average bubble rise velocity, defined relative to the interface motion in the unperturbed simulation, exceeds the Layzer asymptotic rise velocity for an axisymmetric bubble in a semi-infinite fluid $0.36(\mathrm{~g} \lambda)^{1 / 2}$. The differences between the bubble rise velocities obtained from the simulations and from the Layzer formula increase with the initial perturbation amplitude, becoming substantial for capsules with the larger perturbations. These differences are similar in magnitude to the estimates of linear growth rates for the Richtmyer-Meshkov instability induced by the passage of the first rebounding shock. For example, with an initial 1.25- $\mu$ m-deep perturbation, the average bubble rise velocity during the first 100 ps of deceleration is $89 \mu \mathrm{m} / \mathrm{ns}$. The calculated

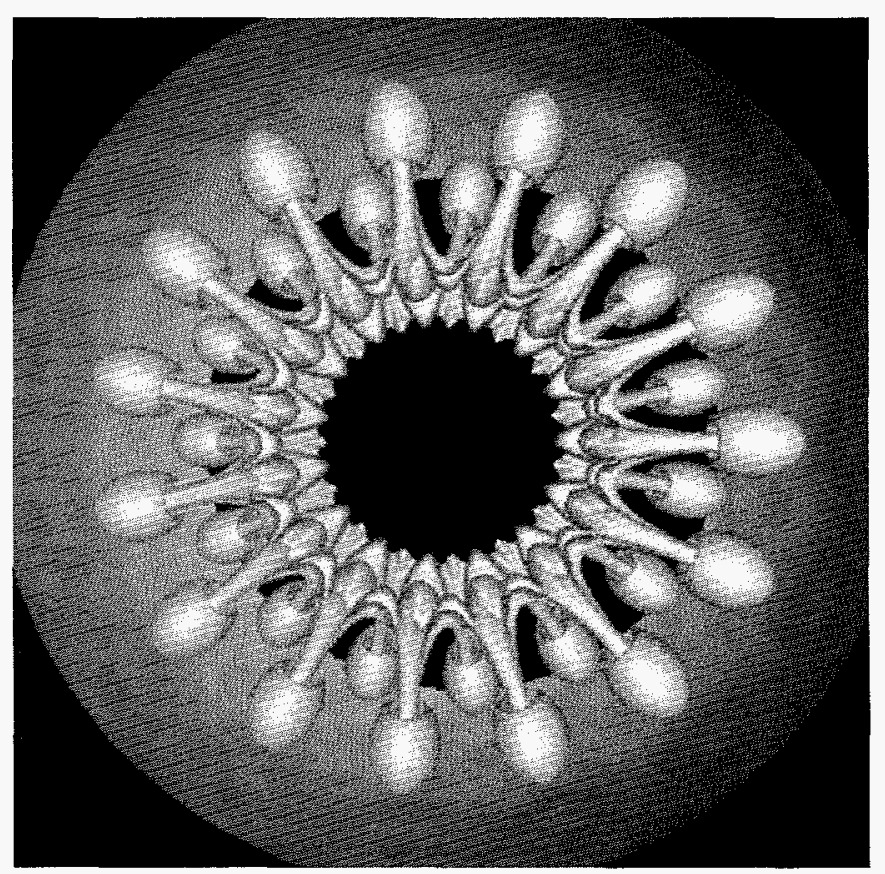

FIGURE 11. Three-dimensional plot of isodensity surface for $\rho=18 \mathrm{~g} / \mathrm{cm}^{3}$ at $2.1 \mathrm{~ns}$ for a Nova capsule with a "soccer ball" perturbation on its exterior. The initial pit depth is $0.25 \mu \mathrm{m}$. Contours are shown over a subset of a hemisphere near the equator. The outer shell is near the ablation region, while the inner surface shown corresponds to the fuel-pusher interface. $\quad(50-05-0595-1118 \mathrm{pb} 01)$ 
Layzer rise velocity is $40 \mu \mathrm{m} / \mathrm{ns}$, and the estimated Richtmeyer-Meshkov growth rate due to the first rebounding shock is $56 \mu \mathrm{m} / \mathrm{ns}$.

Bubble rise velocities are also affected by spherical convergence, expansion of the rising bubbles, finite shell thickness, and the rise through multiple fluid layers. Further increases are seen later in the bubble rise velocities during a period when they encounter a reflected shock rebounding from the center. The Richtmyer-Meshkov instability clearly has a substantial impact on the perturbation growth during deceleration, particularly on the less massive bubbles. For perturbations in the nonlinear regime, the shocks appear to have a less pronounced effect on growth of the more massive spikes.

As discussed earlier, for a given wavelength a perturbation in the shape of an axisymmetric bubble is able to penetrate the capsule shell fastest. For an initial bubble depth of $2.5 \mu \mathrm{m}$, the bubble growth in the ablation layer is sufficient to burn through the capsule by the onset of deceleration. As the capsule material ahead of the bubble thins, the bubble tip accelerates inward, forming a highly elongated shape. Doped polystyrene ablator penetrates the PVA and mandrel layers, which are more dense at that time. The resulting spikes of pusher material encounter the rebounding shock $\sim 50$ ps sooner than does the fuel-pusher interface in the corresponding unperturbed simulation. The trajectory that the unperturbed interface would follow in the absence of deceleration is termed the fall line. For this large perturbation amplitude, the spike penetrates farther inward, approaching the center of the capsule sooner than the fall line. The neutron production rate is greatly reduced once the spike approaches within several micrometers of the center of the hot spot. Overall neutron yield is only $7.8 \%$ of the value obtained for the unperturbed capsule. Nova capsule simulations show perturbation growth causing a large yield degradation only when a spike of pusher material crashes into the center of the hot spot during the period of peak neutron production.

\section{Summary}

We examined 3-D growth of ablative hydrodynamic instabilities for a variety of indirectly driven targets. All cases simulated show linear growth rates that are independent of mode shape. For a single-mode rectilinear perturbation of a given wave number, the 3-D square mode, which is most symmetric, saturates at the largest amplitude. We verified this for indirectly driven foils through simulation and experiment.

Differences in the nonlinear evolution of foils due to perturbation shape are in good quantitative agreement with predictions of the simulations. Of all perturbation shapes, an axisymmetric bubble will burn through a foil the fastest. An isolated axisymmetric spike will attain the largest areal density amplitude.

We simulated a random multimode foil containing perturbations spanning the range of wavelengths of interest for indirect drive. Early in the nonlinear regime, the multimode foil forms broad bubbles and narrow spikes consistent with second-order mode-coupling theory. The onset of multimode saturation is found to be roughly consistent with the prediction of the Haan saturation model. Later, strongly nonlinear interactions lead to an increase in the average width of features. The largest bubbles rise the fastest and continue to grow broader than the initial perturbations.

Simulations of the "soccer ball" capsule show that the positions initially occupied by pits are where fuel bubbles rise during the deceleration phase. A secondary instability at the fuel-pusher interface leads to roll-up at the tips of these bubbles. Most of the neutron production originates in regions that are several micrometers away from any spikes of pusher material. In Nova capsules, mix causes a large reduction in yield only when a spike of pusher material crashes into the capsule center during the time of peak neutron production. 


\section{Notes and References}

1. R. D. Richtmeyer, Commun. Pure Appl. Math. 13, 297 (1960); E. E. Meshkov, Izv. Akad. Nauk SSSR, Mekh. Ahidk. Gaza 5, 151 (1969); and G. Dimonte and B. Remington, Phys. Rev. Lett. 70, 1806 (1993).

2. J. D. Lindl and W. C. Mead, Phys. Rev. Lett. 34, 1273 (1975).

3. J. W. Jacobs and I. Catton, J. Fluid Mech. 187, 353 (1988), and J. W. Jacobs and I. Catton, J. Fluid Mech. 187, 329 (1988).

4. J. P. Dahlburg and J. H. Gardner, Phys. Rev. A 41, 5695 (1990).

5. Y. T. Lee and R. M. More, Phys. Fluids 27, 1273 (1984).

6. R. J. Ellis, J. E. Trebes, D. W. Phillion, J. D. Kilkenny, et al., Rev, Sci. Instrum. 61, 2759 (1990); B. A. Remington, S. G. Glendinning, R. J. Wallace, S. Rothman, and R. Morales, Rev. Sci. Instrum. 63, 5080 (1992); B. A. Remington and R. I. Morales, Rev. Sci. Instrum. 66, 703 (1995).
7. K. S. Budil, T. S. Perry, P. M. Bell, P. L. Miller, et al., "The Flexible X-Ray Imager," Lawrence Livermore National Laboratory, Livermore, CA, UCRL-JC-120837 (1995).

8. B. A. Remington, S. V. Weber, M. M. Marinak, S. W. Haan, et al., Phys. Plasmas 2, 241 (1995).

9. R. J. Wallace, R. L. McEachern, and W. W. Wilcox, ICF Quarterly Report 4(3), 79, Lawrence Livermore National Laboratory, Livermore, CA, UCRL-LR-105821-94-3 (1994).

10. M. J. Dunning and S. W. Haan, ICF Quarterly Report 3(4), 179 Lawrence Livermore National Laboratory, Livermore, CA, UCRL-LR-105821-93-4 (1993).

11. S. W. Haan, Phys. Fluids B 3, 2349 (1991).

12. D. Layzer, Astrophys. J. 122, 1 (1955)

13. S. W. Haan, Phys. Rev. A 39, 5812 (1989). 


\section{Progress Toward a Prototype Recirculating ION INDUCTION ACCELERATOR}

$\begin{array}{lll}\text { A. Friedman } & \text { H. C. Kirbie } & \text { W. M. Sharp } \\ \text { J.J. Barnard } & \text { B. G. Logan } & \text { T. J. Fessenden } \\ \text { M. D. Cable } & \text { S.M. Lund } & \text { D. L. Judd }{ }^{\dagger} \\ \text { D. A. Callahan } & \text { L. A. Nattrass } & \text { S. Eylon } \\ \text { F. J. Deadrick } & \text { M. B. Nelson } & \text { H. A. Hopkins }+ \\ \text { D. P. Grote } & \text { M. A. Newton } & \text { D. B. Longinotti* } \\ \text { V.P. Karpenko } & \text { T.C.Sangster } & \end{array}$

\section{Introduction}

The U.S. Inertial Fusion Energy (IFE) Program is developing the physics and technology of ion induction accelerators, with the goal of electric power production by means of heavy ion beam-driven inertial fusion (commonly called heavy ion fusion, or HIF). Such accelerators are the principal candidates for inertial fusion power production applications, because they are expected to enjoy high efficiency, inherently high pulse repetition frequency (power plants are expected to inject and burn several fusion targets per second), and high reliability. In addition (and in contrast with laser beams, which are focused with optical lenses) heavy-ion beams will be focused onto the target by magnetic fields, which cannot be damaged by target explosions. Laser beams are used in present-day and planned near-term facilities (such as LLNL's Nova and the National Ignition Facility, which is being designed) because they can focus beams onto very small, intensely illuminated spots for scaled experiments and because the laser technology is already available.

LLNL has participated in HIF research since its inception and has worked on HIF target physics and fusion-chamber beam-propagation physics, accelerator physics and technology, and advanced power-plant concepts. Here we discuss a newly initiated experimental effort aimed at developing a low-cost approach

\footnotetext{
†Lawrence Berkeley Laboratory, Berkeley, California

*Titan Beta, Dublin, California

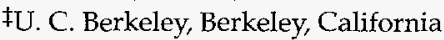

${ }^{* *}$ EG\&G, Inc., Pleasanton, California
}

to high-current heavy-ion acceleration, based on recirculation of the ion beams.

An induction accelerator works by passing the beam through a series of accelerating modules, each of which applies an electromotive force (emf) to the beam as it goes by; effectively, the beam acts as the secondary winding of a series of efficient one-turn transformers. Each of these transformers requires a sizable ferromagnetic toroid, or core. The cores must be large enough to sustain enough volts of emf for long enough to impart the required impulse to the entire beam. In the recirculating induction accelerator, or recirculator, the beam is repeatedly passed through the same set of accelerating cores and focusing elements, thereby reducing the length of the accelerator and the number of cores and magnets required. In a recirculator, it is not necessary to accelerate the beam quickly to minimize length and cost, as is the case in a linear accelerator, or linac; acceleration can be more gradually, over many laps, and this allows the cores to be made smaller and less expensive (the required voltage is lower). This promises a very attractive driver cost if the technical challenges associated with recirculation can be met.

The recirculator concept as it applies to an ICF driver was presented in two previous ICF Quarterly articles, ${ }^{1-2}$ and elsewhere ${ }^{3}$; another recent article ${ }^{4}$ describes LLNL's work on a pulse modulator for use in a recirculator. Figure 1 shows an artist's conception of an IFE power plant driven by an induction recirculator.

We present plans for and progress toward the development of a small (4.5-m-diam) prototype recirculator, which will accelerate singly charged potassium ions 
(atomic weight $39 \mathrm{u}$ ) through 15 laps, increasing the ion energy from 80 to $320 \mathrm{keV}$ and the beam current from 2 to $8 \mathrm{~mA}$. Beam confinement and bending are effected with permanent-magnet quadrupoles and electric dipoles, respectively. The design is based on scaling laws and on extensive particle and fluid simulations of the behavior of the space charge-dominated beam. The dimensionless beam dynamics parameters were chosen to resemble those of a driver; the experiments should serve to validate major elements of the recirculator concept.

This Small Recirculator is being developed in a build-and-test sequence. An injector and matching section are operational, and we are already investigating intense-beam transport in a linear magnetic channel. Near-term plans include studies of space charge-dominated beam transport around a bend. Later we will study insertion and extraction of the beam into and out of the ring, and acceleration with centroid control. The ultimate goal is demonstration of flexible recirculator operation.

In contrast with conventional rf-driven accelerators, the beam in the Small Recirculator experiments is space-charge dominated. That is, the transverse force balance is a near-cancellation of the applied confining force and the beam's self-induced electrostatic expanding force; thermal pressure plays only a minor role. Effectively, the beam is a non-neutral plasma in nearly laminar flow. The fully operational Small Recirculator will confine and accelerate many more ions than a conventional circular accelerator of this scale and beam energy.

\section{Recirculator Concept}

A recirculating induction accelerator is expected to cost considerably less than a conventional ion-induction linac. The overall accelerator length is reducedby a factor of $\sim 2$ to 3 , to about $3.6 \mathrm{~km}$ (and possibly shorter) in the "C-design" recirculator of Ref. 3. The accelerating cores are smaller, because acceleration can be slower. Research on recirculator drivers has centered on four-beam multi-ring designs, in which each ring increases the beam's energy by an order of magnitude over 50 to 100 laps. In contrast with most HIF induction linac concepts, ${ }^{5}$ recirculator designs considered to date do not use beam merging. Hybrid designs (with a recirculator at the low-energy end and a linac at the highenergy end) are also possible and may prove attractive.

The beam-dynamics issues that must be resolved before a recirculating driver can be built include centroid control, longitudinal control, and beam insertion and extraction. Beam compactness must be preserved throughout, so that the beam can eventually be focused onto a small spot. The critical measure in this context is compactness in the 6-D phase space of particle positions and velocities. Accelerator researchers commonly use a measure of compactness called emittance, which is the area occupied by the beam in a two-dimensional projection of that 6-D phase space. For example, the horizontal transverse emittance $\varepsilon_{x}$ is the area occupied by particles in the space $\left(x, x^{\prime}\right)$, where $x$ is the horizontal coordinate normal to $z$ (the mean direction of beam motion) and $x^{\prime}$ is the velocity along that coordinate normalized to the velocity along $z$, i.e., the angle between a particle's velocity and the local mean velocity. For many purposes the normalized emittance $\varepsilon_{\mathrm{n}}=\beta \gamma \varepsilon$ is used, where $\beta$ is the particle speed normalized to the speed of light and $\gamma=\left(1-\beta^{2}\right)^{-1 / 2}$. The normalized emittance enjoys the property of being conserved during acceleration if no nonlinear (with respect to the individual coordinates) forces act on the beam. Of course, some nonlinearity is always present in real accelerators, so some emittance growth will in general occur, but the concept of normalized emittance remains useful.

As described below, beam-dynamics issues can be addressed at reduced scale in a small prototype recirculator. The waveform generators in a driver must supply variable accelerating pulses at repetition frequencies of

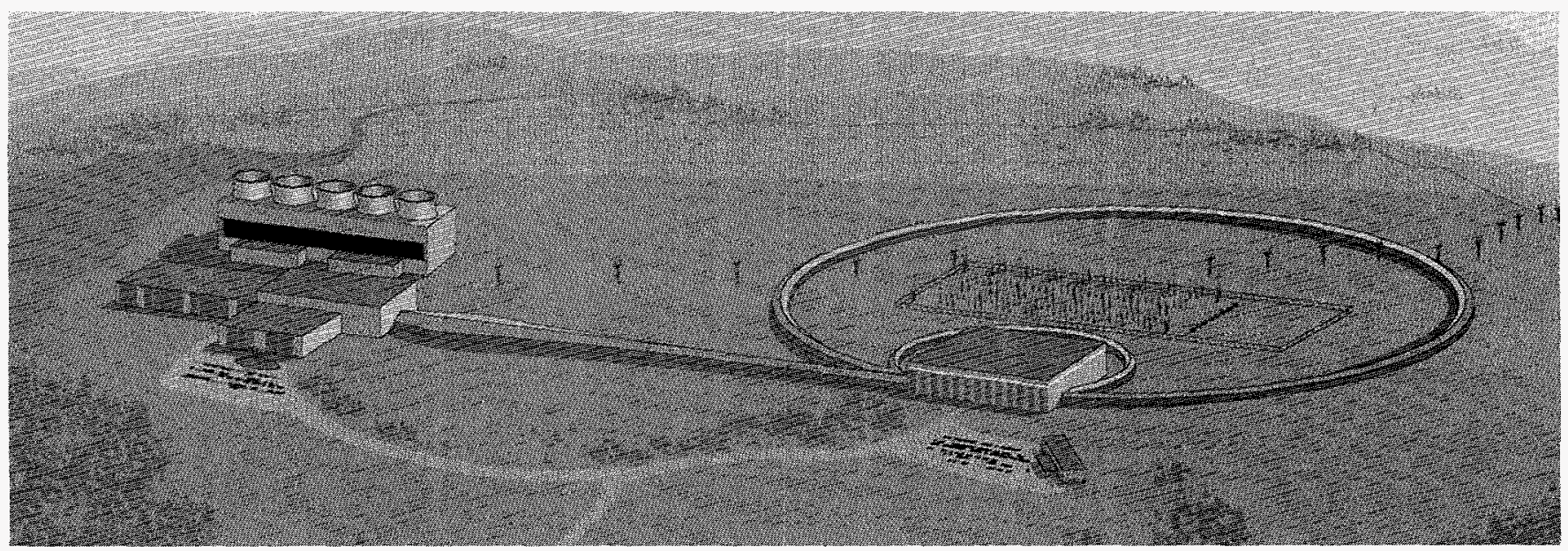

FIGURE 1. Artist's rendering of recirculator-driven power plant. (05-00-1194-3846pb03) 
$\sim 50 \mathrm{kHz}$ and must supply accurate time-varying dipole fields with good energy recovery. These requirements are challenging, but advances in solid state power electronics should make it possible to meet them through a technology development program. Present technology has achieved $200-\mathrm{kHz}$ bursts at $5 \mathrm{kV}$ and $800 \mathrm{~A}$ with pulse durations of 0.5 to $2 \mathrm{~ms}$, but with a nonvariable format. ${ }^{6}$ Because of its long $(\sim 200 \mathrm{~km})$ beam path length, and because the beam repeatedly visits each section of the beam line, a recirculator driver will require a vacuum of $\sim 10^{-10}$ to $10^{-11}$ Torr.

\section{Design of the Small Recirculator}

LLNL is developing a small prototype ion recirculator in collaboration with LBL, EG\&G, and Titan-Beta. This Small Recirculator will be assembled and operated as a series of experiments over several years. Figure 2 illustrates the overall physics design of the Small Recirculator and lists some of the elements that must all work together in the Small Recirculator and in a full-scale fusion driver.

The Small Recirculator will have a circumference of $14.4 \mathrm{~m}$, a $3.5-\mathrm{cm}$ aperture (pipe) radius for the beam-

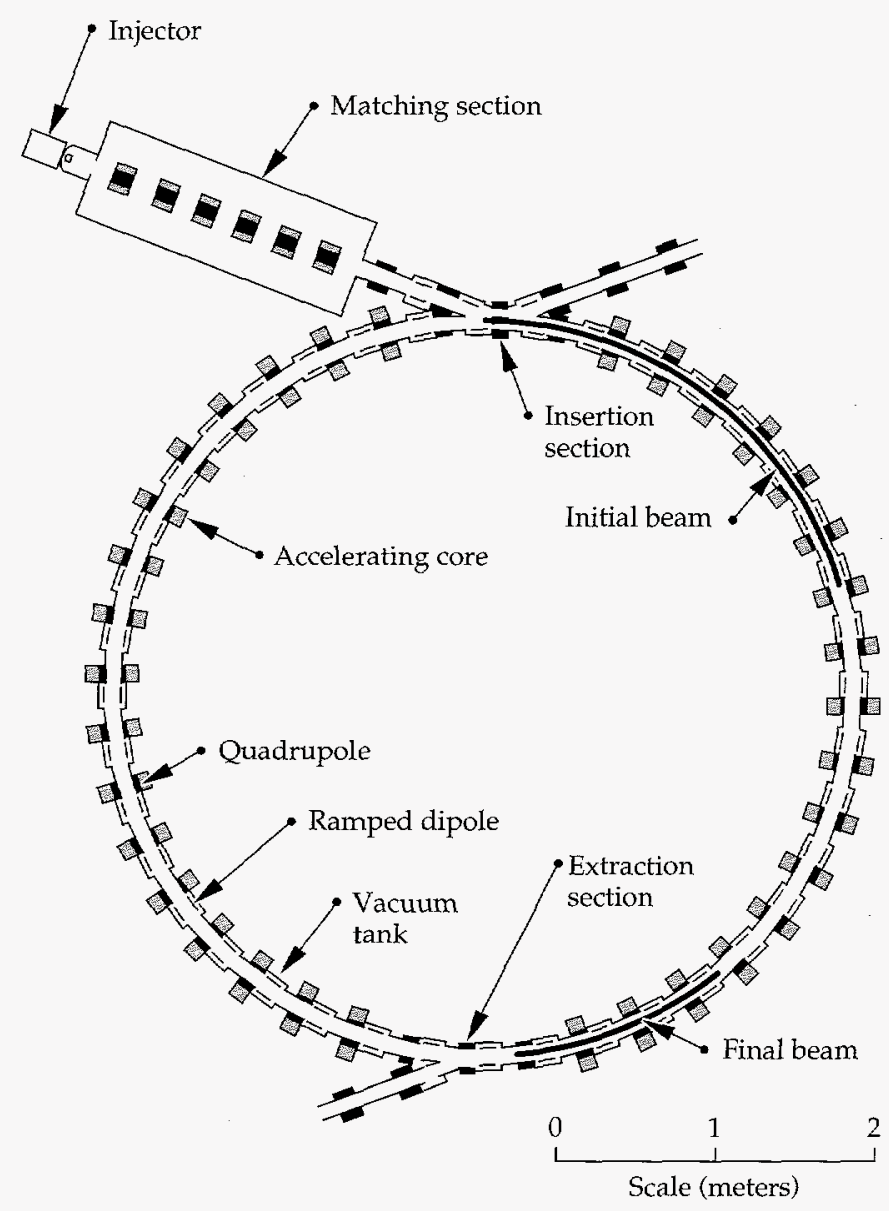

FIGURE 2. Final configuration of the Small Recirculator. (30-00-0695-1644pb01) confining (commonly called focusing) and beam-bending elements, and a $72-\mathrm{cm}$ lattice period (segment of the repetitive lattice of focusing and bending elements). The beam will be transversely confined by permanentmagnet quadrupole lenses with a field of $\sim 0.294 \mathrm{~T}$ at the pipe wall, and will be bent with electric dipole deflector plates. These quadrupoles and dipoles will each physically occupy about $30 \%$ of the axial lattice length, and the full recirculator ring will consist of 40 half-lattice periods, including one or two periods using special large-aperture quadrupole magnets through which the beam will be inserted and/or extracted. The fundamental building block is actually the $36-\mathrm{cm}$ half-lattice period, but the polarity of the quadrupole lenses is reversed in each alternate half-lattice period; this provides so-called alternating-gradient or strong focusing as in most modern particle accelerators.

The $\mathrm{K}^{+}$beam ions will be accelerated from an initial kinetic energy of $80 \mathrm{keV}$ to $320 \mathrm{keV}$ over 15 laps by 34 induction cores (no induction cores will be present in the lattice periods where the beam is inserted and extracted). The initial beam current will be $2 \mathrm{~mA}$, corresponding to a line-charge density of $3.6 \mathrm{nC} / \mathrm{m}$ and a characteristic beam radius of $1.1 \mathrm{~cm}$, and the initial pulse duration will be $4 \mu \mathrm{s}$. After 15 laps of acceleration, the beam current will have increased to $8 \mathrm{~mA}$, the line-charge density will be $7.21 \mathrm{nC} / \mathrm{m}$, the average beam radius will be $1.3 \mathrm{~cm}$, and the pulse duration will be 1 rs.

Because the quadrupole magnets provide a transverse restoring force to confine the beam, the beam centroid will, if displaced off-axis, oscillate back and forth across the centerline of the beam pipe. Over a full oscillation, the phase of the displacement will sweep through $360^{\circ}$. The initial phase advance of these "betatron oscillations" per lattice period of beam motion will be $\sigma_{0}=78^{\circ}$. Thus a beam-centroid oscillation will require $360^{\circ} / 78^{\circ} \approx 4.6$ lattice periods, or about $4.6 / 20=23 \%$ of the circumference of the ring. Individual particles also execute betatron oscillations back and forth within the confines of the beam, but the frequency of these oscillations is lower than the "undepressed" frequency because the net focusing force is reduced by the repulsive effects of space charge, which (if unopposed) would blow up the beam. Initially, the net effect is a phase advance depressed to $\sigma=16^{\circ}$ by space charge. After 15 laps of acceleration, the phase advances will decrease to $\sigma_{0}=45^{\circ}$ and $\sigma=12^{\circ}$. These parameters were chosen to resemble those of a driver-scale recirculator, although of course the latter would have many more betatron oscillations per lap.

Because the heavy-ion beam in the Small Recirculator is nonrelativistic and accelerating, obtaining the variable-format accelerating and bending waveforms will be technologically challenging. Those waveforms will require the accurate synthesis of detailed voltage pulses with repetition rates rising from about $40 \mathrm{kHz}$ at the 
initial beam energy to $90 \mathrm{kHz}$ at the final beam energy. 4,7 The voltage pulses for the electric dipoles must be correctly ramped in concert with the increasing beam energy. Properly shaped "ear" pulses must be applied at the beginning and end of the main pulses to provide longitudinal confinement, and lap-to-lap variation of the pulse duration and shape must be added to the accelerating waveforms to maintain or decrease the beam length.

To switch the beam into or out of the ring, timevarying dipole (bending) fields must be applied. Transverse confinement of the beam must be carried out during insertion or extraction. Our design uses a permanent-magnet quadrupole with an expanded aperture. Figure 3 shows the physics design of the insertion/extraction section. The main ring runs along the lower part of the figure; the insertion line (which brings the beam from the matching section into the ring) comes in from the upper left, and the extraction line runs toward the upper right. The beam trajectory is shown as it will appear during extraction.

Mechanical design of the Small Recirculator was challenging because of the necessity of fitting bending, focusing, and accelerating elements, as well as provisions for vacuum pumping and beam diagnostics, into each half-lattice period. Figures 4 and 5 show the nearly complete computer-aided design, as rendered by the CAD software.

\section{Recirculator Modeling}

Because the space charge-dominated beams in an induction accelerator are effectively non-neutral plasmas, theoretical and computational modeling of these beams is carried out using techniques related to those used in the accelerator and plasma physics communities.
Models used range from simple zero-dimensional codes based on analytically derived scaling relations, through fluid- and moment-equation simulations, up to large and elaborate discrete-particle simulations.

The CIRCE code $^{8}$ is a multidimensional model that solves an envelope equation (evolving moments such as centroid position and transverse extent) for each of a number (typically a hundred or greater) of transverse beam "slices," each at different longitudinal positions. The longitudinal dynamics of the beam is modeled by evolving the positions and velocities of the slices using fluid equations. CIRCE is used to assess alignment tolerances, accelerating schedules, and steering techniques in linacs and recirculators. It is useful for any application in which the evolution of the detailed internal degrees of freedom of the beam (e.g., emittance growth processes) need not be resolved; at present, beam normalized emittance is assumed constant in CIRCE.

Because the beam resides in the accelerator for relatively few plasma oscillation periods, particle-in-cell

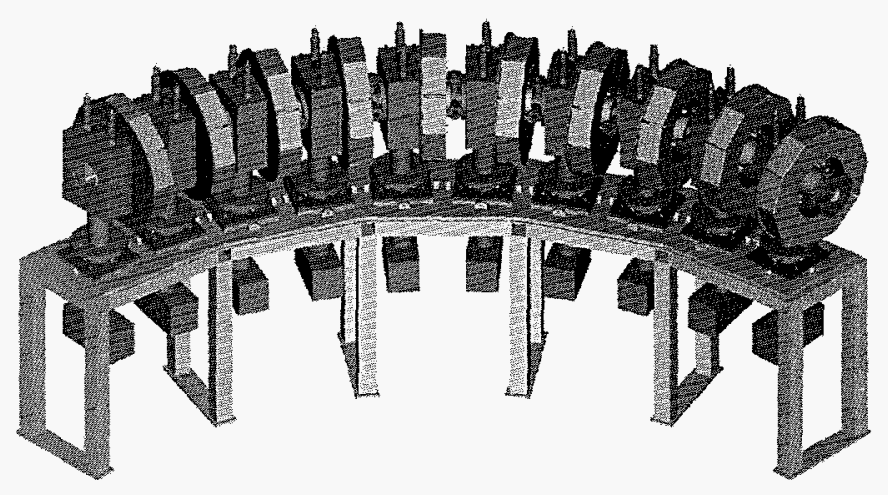

FIGURE 4. One-quarter of the Small Recirculator (CAD rendering). (30-00-0695-1649pb01)
FIGURE 3. Insertion/ extraction section. (30-00-0695-1646pb01)

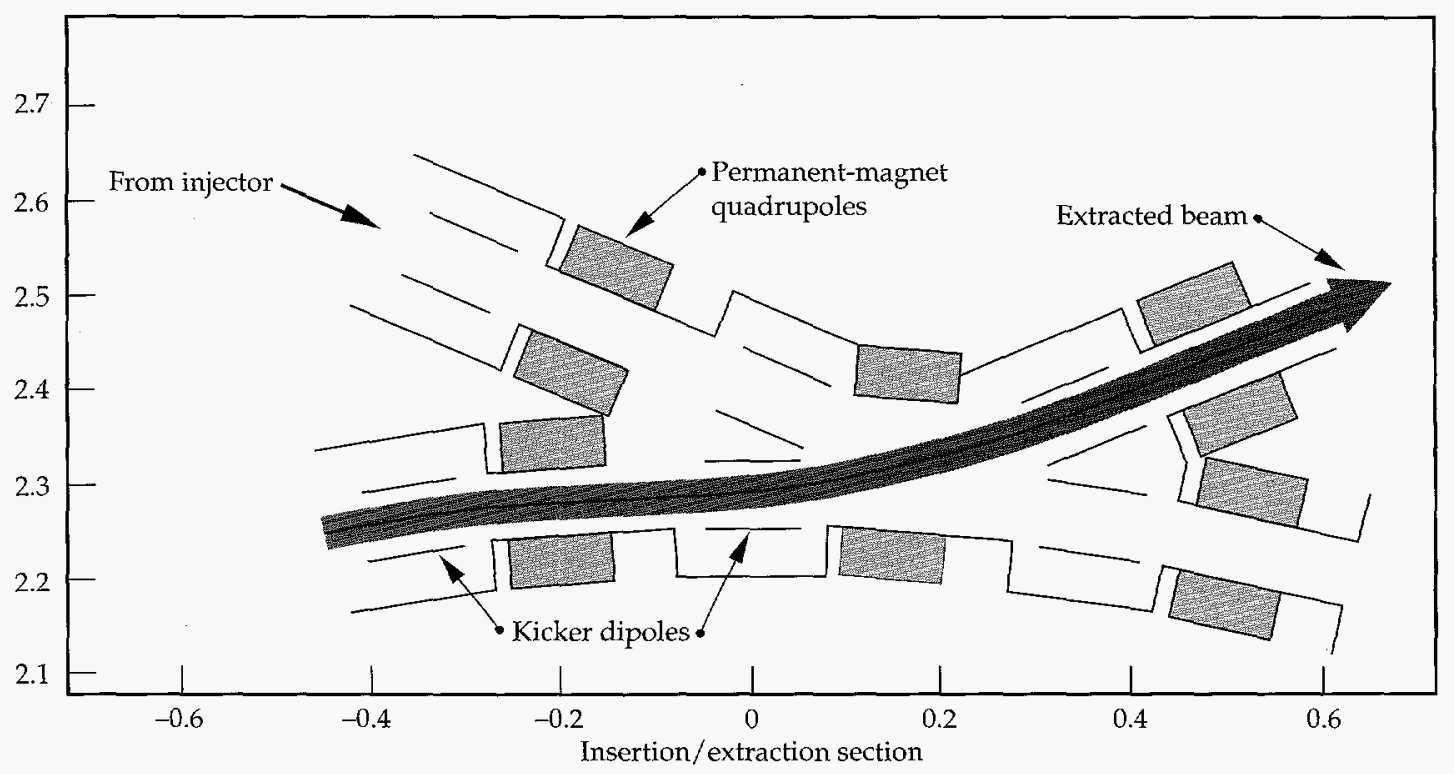


(PIC) simulation techniques are especially effective and have been invaluable in the design and analysis of experiments and in the prediction of the behavior of future machines. The WARP code includes fully threedimensional (WARP3d) ${ }^{9}$ and axisymmetric (WARPrz) ${ }^{10}$ PIC simulation models. WARPrz is used for long-term beam dynamics studies, including the effects of accelerating module impedance. WARP3d is heavily used for "near-first principles" studies of accelerator elements and experiments.

Several novel techniques make WARP3d both accurate and efficient. These include capabilities for subgridscale placement of internal conductor boundaries and for simulating "bent" accelerator structures, as well as a technique for rapidly following particles through a sequence of sharp-edged accelerator lattice elements, using a relatively small number of time steps while preserving accuracy. On some problems, WARP3d is run in a quasi-steady state mode, which permits the completion of a 3-D run in just a few minutes of computer time; this makes it suitable for iterative design calculations. The ultimate goal of this code development is effective simulation of present-day experiments and of an HIF driver, from the source through the final

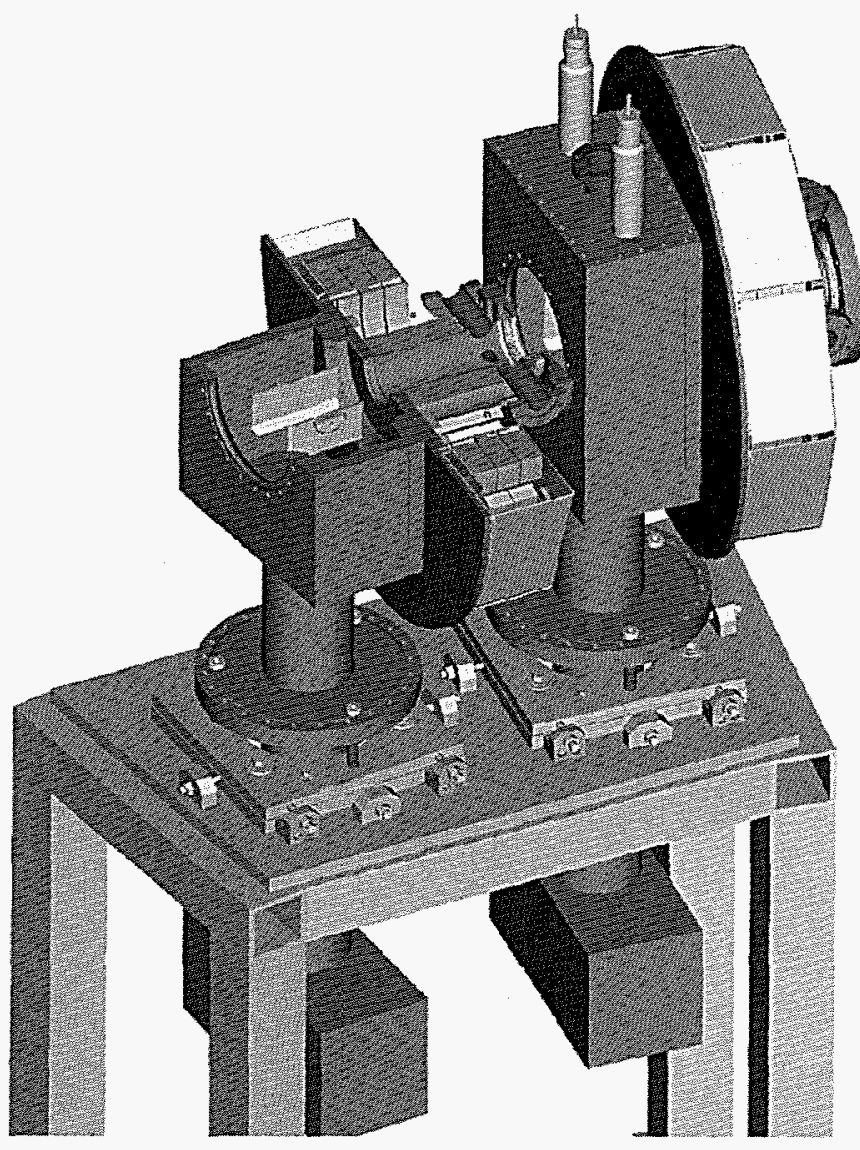

FIGURE 5. Two half-lattice periods of the Small Recirculator (CAD rendering). The top of the left-hand half-lattice period is cut away to reveal the internal structure. $\quad(30-00-0695-1650 \mathrm{pb01})$ focus, with a link from WARP into the codes used to model propagation in the fusion chamber and ultimately into the target design codes.

A number of other PIC codes employ a "slice" description of the beam (assuming slow variation of quantities along the beam); much application, as well as detailed studies of the properties of such PIC models of beams and plasmas, has been carried out. ${ }^{11}$

Emittance growth can result from the nonuniform distribution of beam space charge resulting from the action of centrifugal forces. As revealed in particle simulations using WARP $3 \mathrm{~d}^{9}$ and interpreted theoretically, ${ }^{12}$ growth occurs at changes in the accelerator's curvature where the distribution of beam particles relaxes toward a new equilibrium. A circular recirculator is therefore to be preferred over one with an elongated "racetrack" shape. Since the Small Recirculator is effectively circular (the changes of curvature that occur within a single half-lattice period are too rapid to matter), the only significant changes in curvature occur during insertion and extraction. The electric dipoles also introduce field aberrations. Detailed 3-D simulations show that proper shaping of the dipole plates should render the beam distortion minimal. We have studied the behavior of the beam in the Small Recirculator in some detail using CIRCE and WARP. A measurable amount of emittance growth is expected to take place over the 15 laps, mostly in the first two laps. ${ }^{13}$

Here we show the results of a WARP3d simulation of the beam in the Small Recirculator. For reasons of economy, the simulated beam is often made shorter than the actual beam will be. Figure 6 shows top and side views of the beginning of the simulation; the final

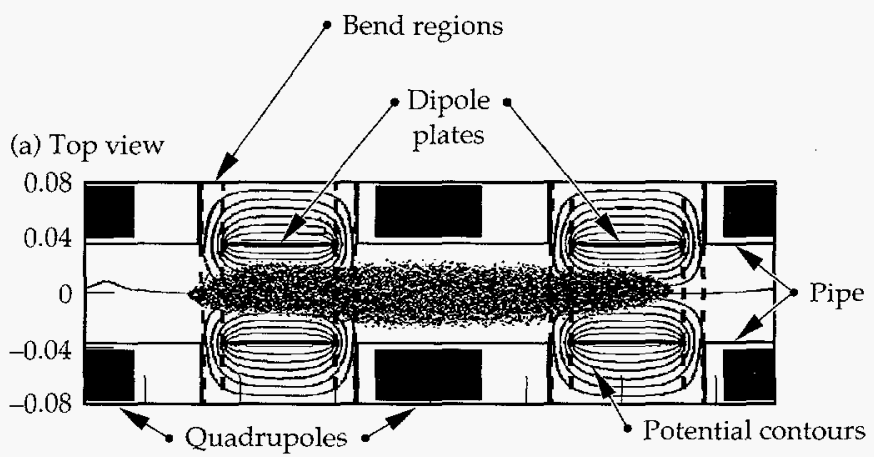

(b) Side view

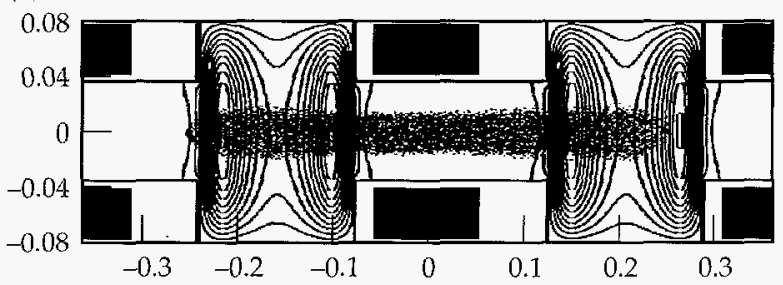

FigURE 6. Top and side views of Small Recirculator beam simulated using WARP3d. "Bends" are shown straightened. (30-00-0695-1656pb01) 
beam is very similar. Figure 7 shows the evolution of the emittance at mid-pulse; in this case, the beam length is kept roughly constant. After the initial jump arising from the transition to the bent lattice, there is little emittance growth over the 15 laps.

\section{Experiment Plans}

Linear experiments now getting under way will measure space charge-dominated beam quality after transport through a permanent-magnet quadrupole lattice, characterize the beam before injection, provide a test bed for diagnostic development, and afford a preliminary assessment of the role of electrons in magnetic beam transport (see Fig. 8).

The next experiments will study beam transport around a bend of order $90^{\circ}$ (at first without any accelerating modules). The transition of the beam from a straight transport line into the ring will represent a

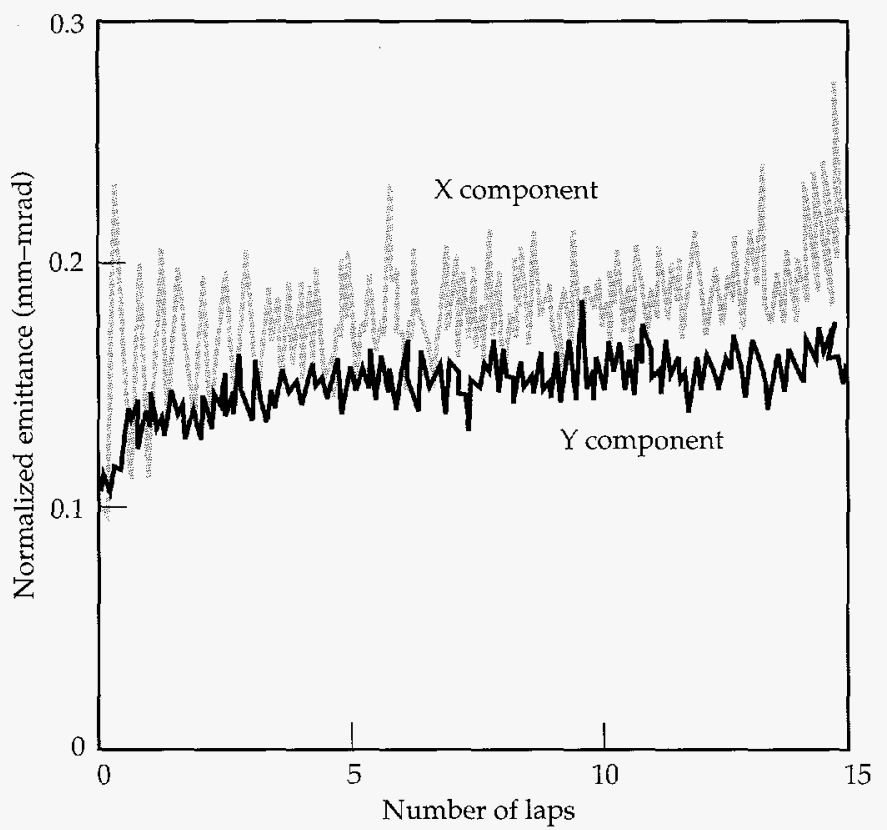

FIGURE 7. Time dependence of normalized beam emittance at mid-pulse for $X$ (in-plane) and $Y$ (out-of-plane) components. (30-00-0695-1658pb02)

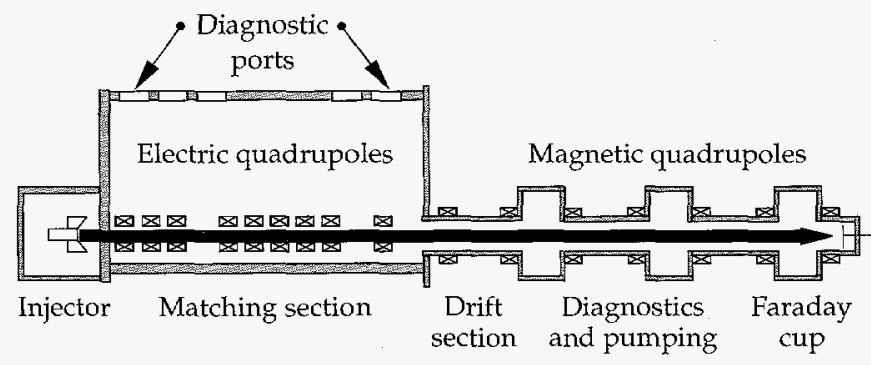

FIGURE 8. Linear configuration (length $4.568 \mathrm{~m}$ from source to final Faraday cup). (30-00-0695-1657pb01) change of curvature, and will allow us to study the resulting emittance growth. Emittance growth can also result from imperfections in the focusing and bending fields; the small imperfections expected in our experiments will be well characterized by theory and measurement. Even over a short bend, detailed intercepting beam diagnostics (using a two-slit apparatus to measure both transverse ion position and velocity) should be able to detect relatively small changes in the distribution of beam particles as a result of the bend. An important goal of these initial experiments will be validation of the computer models and scaling laws used to predict the behavior of linear and recirculating drivers.

Later experiments will study insertion and extraction, acceleration (at first in a partial ring to facilitate measurement of the beam using intercepting diagnostics), beam steering, bunch compression, and fully integrated operation of the recirculator. Preservation of a small emittance will again be the central beam-physics issue to be addressed.

Until the ring is complete it will be possible to use intercepting diagnostics to characterize the beam and to calibrate the nonintercepting diagnostics that will be critical to the successful operation of the full ring. As currently planned, the ring will incorporate two extraction sections $180^{\circ}$ apart, so the extracted beam. can be diagnosed with detailed intercepting diagnostics twice each lap. As with earlier linac experiments at LBL, excellent shot-to-shot repeatability is anticipated and, so far, observed. The principal nonintercepting diagnostic under development is a segmented capacitive pickup to be located inside the quadrupoles. ${ }^{14}$ The long duration of beam residence in the machine (up to, and possibly exceeding, 300 full lattice periods) will provide a unique opportunity to observe and characterize the longitudinal propagation of space-charge waves along the beam. Such waves will be launched (deliberately or otherwise) by mismatching the applied ear fields. The Small Recirculator will afford the longest beam path length of any near-term HIF research facility, and so will be able to explore issues such as slow thermalization that are important to both recirculating and linear drivers.

\section{Status and Initial Results}

The injector diode, ${ }^{15}$ matching section, and straight experiment have been fabricated and are now operating; Fig. 8 shows the layout. Fifteen permanent-magnet quadrupoles have been procured; seven are being used in the straight experiment (see Fig. 10). A shorter line will serve as the link from the matching section to the ring. As shown in Figs. 4 and 5 , the mechanical design of the half-lattice period is nearly complete. ${ }^{16}$ 
The electrostatic-quadrupole matching section (Fig. 8) gives the circular beam that leaves the diode an elliptical cross section suitable for alternating-gradient transport in the transfer line and the recirculator. A section of the Single Beam Transport Experiment (SBTE) apparatus from LBL was adapted by EG\&G to serve this function. The potentials applied to the various quadrupole elements to obtain a matched beam were derived using an envelope calculation and range from \pm 1.8 to $\pm 4.0 \mathrm{kV}$. The fifth and seventh elements are intended for minor beam steering rather than for focusing. Insertable Faraday cups are located after the third and ninth elements.

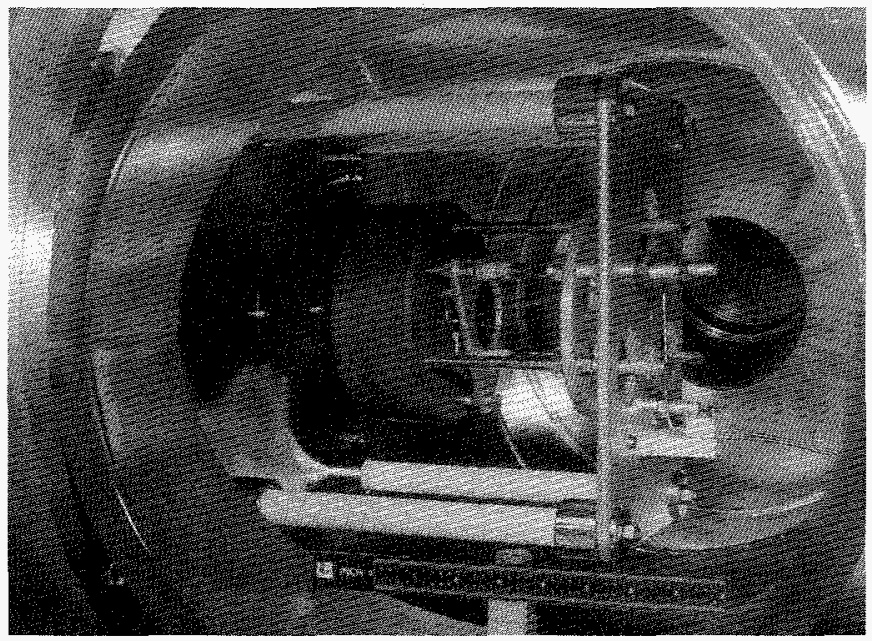

FIGURE 9. Interior of source tank, showing back of source and (at left) hole through which beam passes on its way into matching section. Holes of varying size can be used. (30-00-0695-1653pb01)

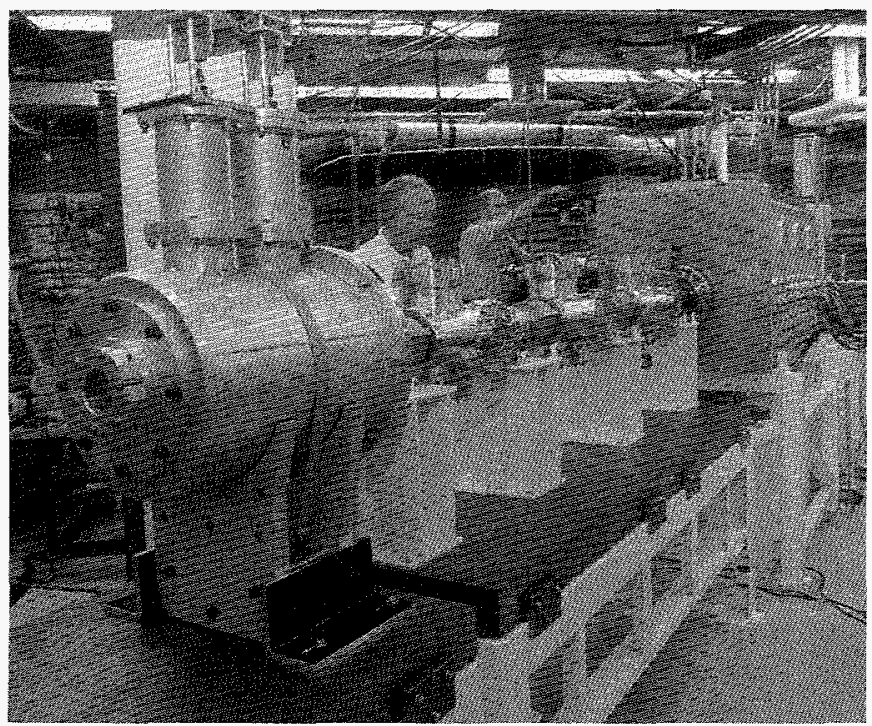

FIGURE 10. Straight transport section. Each spool holds two permanent-magnet quadrupoles; the entire section uses seven quadrupoles. The large enclosure at the left foreground contains diagnostic apparatus. (Orientation is opposite to that of Fig. 8, which does not show diagnostics enclosure.) (30-00-0695-1651pb01)
Figures 9 and 10 show some of the experimental apparatus. Figure 9 shows the source and diode. A potassium-impregnated zeolite element is heated by an internal filament; the beam passes through the hole visible at the left end of the anode-cathode gap. Figure 10 shows the linear transport section.

Time-resolved measurements of beam properties have been obtained at various locations throughout the matching and magnetic transport sections. The current has been measured using Faraday cups 0.67 and $1.9 \mathrm{~m}$ downstream from the diode source in the matching section and $3.16 \mathrm{~m}$ downstream in the magnetic transport section. An energy analyzer developed at LBL (consisting of curved electrostatic plates across which various potential differences are placed) was located $1.75 \mathrm{~m}$ downstream from the source. A two-slit scanner was placed at positions 0.2 and $1.6 \mathrm{~m}$ downstream from the source, providing measurements of emittance, beam radius, and beam centroid location.

Figure 11 shows an example of current vs time at the Faraday cup $1.9 \mathrm{~m}$ downstream from the diode source and corresponding results from the 1-D code $\mathrm{HINJ}^{17}$; there is close agreement between simulation and experiment. The large current spike at the head of the pulse arises because the rise time of the diode voltage (about $1 \mu \mathrm{s})$ is longer than the ideal rise time of $0.48 \mu \mathrm{s} .{ }^{18}$ With the longer rise time, particles emitted at the beginning of the pulse have significantly lower energy than particles emitted later, so particle overtaking occurs. A modification of the pulser circuitry to reduce the rise time by a factor of two is planned. The code results are slightly noisier than those from the experiment; this results from a numerical deconvolution of the voltage waveform (to account for time lags in the voltage monitor), which introduces noise into the voltage waveform used by the code. Figure 12 shows a measurement of the horizontal normalized emittance at the end of the matching section. The high initial value appears to be due to the instantaneously high line-charge density.

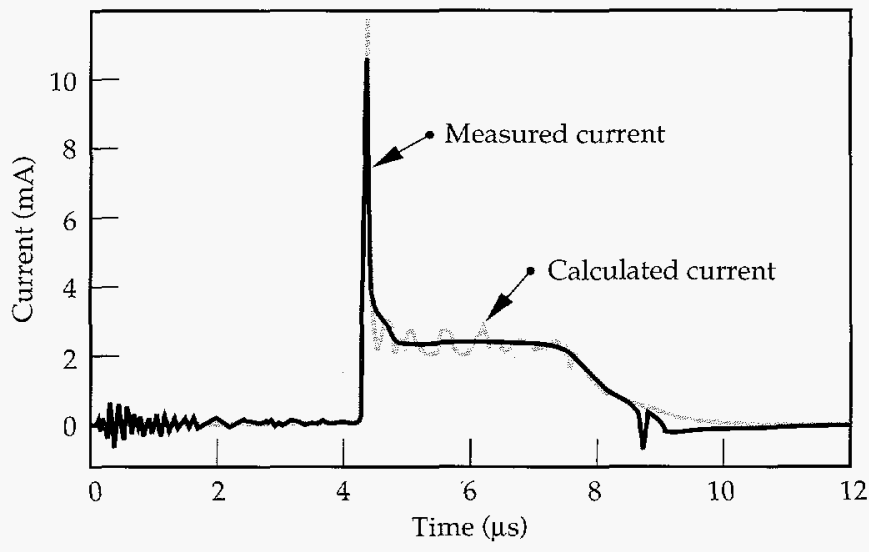

FIGURE 11. Current at the Faraday cup $1.9 \mathrm{~m}$ downstream of the diode source, and corresponding results from the 1-D code HINJ. (30-00-0695-1647 $\mathrm{pb} 01)$ 


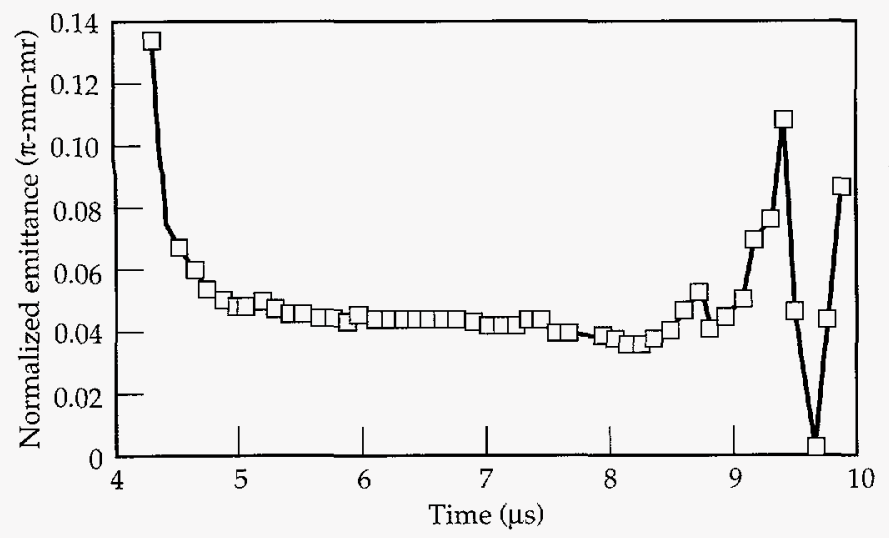

FIGURE 12. Measurement of horizontal normalized emittance at end of matching section. (30-00-0695-1648pb01)

\section{Conclusions}

The linear induction accelerator remains a very attractive Inertial Fusion Energy driver; the recirculator offers the promise of significant cost reduction, if the technical challenges of recirculation can be met. The LLNL Small Recirculator experiments are designed to provide a test bed for the necessary technology and to show that the beam dynamics in an induction recirculator are favorable; these experiments will pave the way to a larger-scale follow-on facility. These experiments, in conjunction with the ILSE experimental program at LBL and with detailed computer simulations, will lead to much more precise and credible predictions of heavy-ion driver behavior and cost and will allow the staged construction of the first IFE driver to begin shortly after ICF ignition is demonstrated on the National Ignition Facility.

\section{Notes and References}

1. J. J Barnard, F. J. Deadrick, A. Friedman, D. P. Grote, et al., ICF Quarterly Report 3(1), 41-49, Lawrence Livermore National Laboratory, Livermore, CA, UCRL-LR-105821-93-1 (1993).

2. W. M. Sharp, ICF Quarterly Report 4(2), 70-77, Lawrence Livermore National Laboratory, Livermore, CA, UCRL-LR105821-94-2 (1994).

3. J. J Barnard, F. J. Deadrick, A. Friedman, D. P. Grote, et al., Phys. Fluids B: Plasma Physics 5, 2698 (1993).

4. H. C. Kirbie, R. L. Hanks, S. A. Hawkins, B. C. Hickman, et al., ICF Quarterly Report 5(2), 103-109, Lawrence Livermore National Laboratory, Livermore, CA, UCRL-LR-105821-95-2 (1995).

5. R. O. Bangerter, Il Nuovo Cimento 106 A (11), 1445-1457 (1994).

6. H. C. Kirbie, W. R. Cravey, S. A. Hawkins, M. A. Newton and C. W. Ollis, "A FET-Switched Induction Accelerator Cell," Proceedings of the 9th IEEE International Pulsed Power Conference, Vol. 1 (Institute of Electrical and Electronic Engineers, New York, IEEE Cat. No. 93CH3350-6, 1993), p. 415.

7. T. J. Fessenden, D. P. Grote, and W. M. Sharp, "Accelerator Waveform Synthesis and Longitudinal Beam Dynamics in a Small Induction Recirculator," Proceedings of the 1995 Particle Accelerator Conference, Dallas, TX, May 1-5, 1995 (to be published).

8. W. M. Sharp, J. J. Barnard, D. P. Grote, S. M. Lund, and S. S. Yu, "Envelope Model of Beam Transport in ILSE," Proceedings of the 1993 Computational Accelerator Physics Conference, Pleasanton, CA, February 22-26, 1993 (AIP Conf. Proc. Vol. 297), R. D. Ryne, Ed., pp. 540-548.

9. A. Friedman, D. P. Grote, and I. Haber, Phys. Fluids B: Plasma Physics 4, 2203 (1992).

10. D. A. Callahan, A. B. Langdon, A. Friedman, and I. Haber, "Longitudinal Beam Dynamics for Heavy Ion Fusion," Proc. 1993 IEEE/APS Part. Accel. Conf., IEEE Cat. No. 93CH3279-7 p. 730 (1993).

11. I. Haber and H. Rudd, "Numerical Limits on P.I.C. Simulation of Low Emittance Transport," Proc. Conf. on Linear Accelerator and Beam Optics Codes, La Jolla Inst., (AIP Conf. Proc., Vol. 177, AIP, NY, 1988), C. R. Eminhizer, Ed., p. 161.

12. J. J. Barnard, H. D. Shay, S. S. Yu, A. Friedman, and D. P. Grote, "Emittance Growth in Heavy Ion Recirculators," Proc. 1992 Linear Accel. Conf., Ottawa, Ontario, Canada, AECL-10728, 229 (1992).

13. D. P. Grote A. Friedman, and I. Haber, "Three Dimensional Simulations of a Small Induction Recirculator Accelerator," Proceedings of the 1995 Particle Accelerator Conference, Dallas, TX, May 1-5, 1995 (to be published).

14. F. J. Deadrick, J. J. Barnard, T. J. Fessenden, J. Meredith, J. Rintamaki, "Development of Beam Position Monitors for Heavy Ion Recirculators," Proceedings of the 1995 Particle Accelerator Conference, Dallas, TX, May 1-5, 1995 (to be published).

15. S. Eylon, E. Henestroza, and F. J. Deadrick, "K+ Diode for the Heavy Ion LLNL Recirculator Accelerator Experiment," Proceedings of the 1995 Particle Accelerator Conference, Dallas, TX, May 1-5, 1995 (to be published).

16. V. P. Karpenko, J. J. Barnard, F. J. Deadrick, A. Friedman, et al., "Mechanical Design of Recirculating Induction Accelerator Experiments for Heavy-Ion Fusion," Proceedings of the 1995 Particle Accelerator Conference, Dallas, TX, May 1-5, 1995 (to be published).

17. J. J. Barnard, G. C. Caporaso, and S. S. Yu, "1-D Simulations of Heavy-Ion Injectors," Proceedings of the 1993 Particle Accelerator Conference, Washington, DC, May 17-20, 1993, Vol. 1, p. 712.

18. M. Lampel and M. Tiefenback, Appl. Phys. Lett. 43, 57 (1983). 


\title{
High-EFFicienCy MUltilayer-Dielectric DIFFRACTION GRATINGS
}

\author{
M.D.Perry $\quad$ D. Decker \\ R. D. Boyd $\quad$ B.W. Shore
}

J. A. Britten

\section{Introduction}

The ability to produce short laser pulses of extremely high power and high irradiance, as is needed for fast ignitor ${ }^{1}$. research in inertial confinement fusion, places increasing demands on optical components such as amplifiers, lenses, and mirrors that must remain undamaged by the radiation. Amplifiers pose particular problems, because as pulses become shorter it becomes increasingly difficult to extract the stored energy without causing detrimental effects originating from the dependence of the refractive index $n=n_{0}+n_{2} E^{2} I=n_{0}+\gamma I$ on irradiance $I$. The higher refractive index in the center of an intense laser beam acts as a focusing lens. The resulting wavefront distortion, left uncorrected, eventually leads to catastrophic filamentation. Major advances in energy extraction and resulting increases in focused irradiance have been made possible by the use of chirped-pulse amplification (CPA), long used in radar applications and newly applied to optical frequencies. ${ }^{2}$

Optical-frequency CPA systems, typified by the schematic diagram in Fig. 1, begin with a mode-locked oscillator that produces low-energy seed pulses with durations of ten to a few hundred femtoseconds. As a result of the classical uncertainty relation between time and frequency, these short pulses have a very broad frequency distribution. A pair of diffraction gratings (or other dispersive elements) lengthens the laser pulse and induces a time-varying frequency (or chirp). Following amplification, diffraction gratings compress the pulse back to nearly the original duration. Typically a nanojoule, femtosecond pulse is stretched by a factor of several thousand and is amplified by as much as 12 orders of magnitude before recompression. By producing the short pulse only after amplification, this technique makes possible efficient extraction of energy from a variety of broadband solid state materials, such as Ti:sapphire, ${ }^{3,4}$ alexandrite, ${ }^{5} \mathrm{Cr}$ :LiSAF, ${ }^{6,7}$ and Nd:glass. ${ }^{8,9}$

\section{Constraints on Gratings for CPA}

Achieving high focused irradiance from a pulse ultimately requires both high peak power and excellent beam quality. There is therefore a demand for diffraction gratings that produce a high-quality diffracted wavefront, have high diffraction efficiency, and exhibit a high threshold for laser damage. With careful attention to control of linear and nonlinear aberrations in the lasing medium, and with the addition of a deformable mirror to the beam transport system to correct for other wavefront aberrations, it should be possible to produce nearly diffraction-limited petawatt pulses.

The grating pairs used for pulse compression are typically planar reflection gratings that direct the firstorder $(m=-1)$ diffraction nearly back along the incident direction, as occurs at the Littrow angle. ${ }^{10}$ It is important that the gratings send almost all of the radiation into this order. The need for high efficiency follows from the fact that the beam is diffracted by a grating four times in a typical double-pass pulse compressor. Thus a grating whose diffraction efficiency is $\eta$ gives a maximum compressor efficiency (for double-pass compression) of $\eta^{4}$. As a result of this quartic dependence, even a small increase in grating efficiency provides a large increase in the energy output from the pulse compressor. With a typical diffraction efficiency into the $m=-1$ order of $86 \%$, the maximum compressor efficiency is approximately $55 \%$. By increasing the grating efficiency to $95 \%$, one can achieve compressor efficiencies of over $80 \%$. We have recently achieved nearly this theoretical limit with a record double-pass compressor throughput of $78 \%$.

Commercial gratings ${ }^{11}$ are often replicas, in which a master grating structure is reproduced on a secondary substrate and then given a thin metal coating to achieve high diffraction efficiency. High-quality master gratings for optical frequencies are produced mechanically, by ruling the grating pattern into a metal blank, or 
holographically, by exposing a substrate coated with photoresist to a stable interference pattern, developing the latent image, and overcoating with a metal layer. Carefully designed metal-coated master and replica gratings have achieved diffraction efficiencies as high as $95 \%$ in the $m=-1$ order. ${ }^{12,13}$ High-quality commercial metallic gratings more commonly have diffraction efficiencies of 80 to $92 \%$.

For high-power lasers, the damage threshold of a grating is as important as its diffraction efficiency. In pulse compression, the damage threshold limits the amount of energy that can be tolerated in the pulse for a given grating area. The low damage threshold of diffraction gratings is responsible for their limitation to use in low-power tunable oscillators. Narrow-linewidth, grating-cavity laser systems based on broadband solid state materials such as Ti:sapphire, alexandrite, and Cr:LiSAF require gratings exhibiting damage thresholds above $2 \mathrm{~J} / \mathrm{cm}^{2}$ to access the high energy storage capacity of these materials.
Unfortunately, metallic diffraction gratings, whether produced by mechanical ruling or holographic techniques, have an inherently low threshold for optical damage due to optical absorption. Energy deposited into a thin surface layer (the skin depth) will raise the surface temperature, eventually to the boiling point, unless it is conducted away more rapidly than it arrives. The theoretical damage threshold ${ }^{12}$ for metallic (gold) gratings operating in the region $700-1100 \mathrm{~nm}$ is less than $1.5 \mathrm{~J} / \mathrm{cm}^{2}$ for nanosecond pulses and less than $0.6 \mathrm{~J} / \mathrm{cm}^{2}$ for picosecond pulses. Our holographically produced gold-coated master gratings ${ }^{12}$ exhibit damage thresholds for 1053-nm radiation of approximately $1.2 \mathrm{~J} / \mathrm{cm}^{2}$ for nanosecond pulses and $0.4 \mathrm{~J} / \mathrm{cm}^{2}$ for subpicosecond pulses. These values are near the theoretical maximum and are a factor of two higher than those available with commercial gratings.

Dielectric materials offer the potential for significantly higher optical damage thresholds than those of metals. At a laser wavelength of $1053 \mathrm{~nm}$, fused silica exhibits

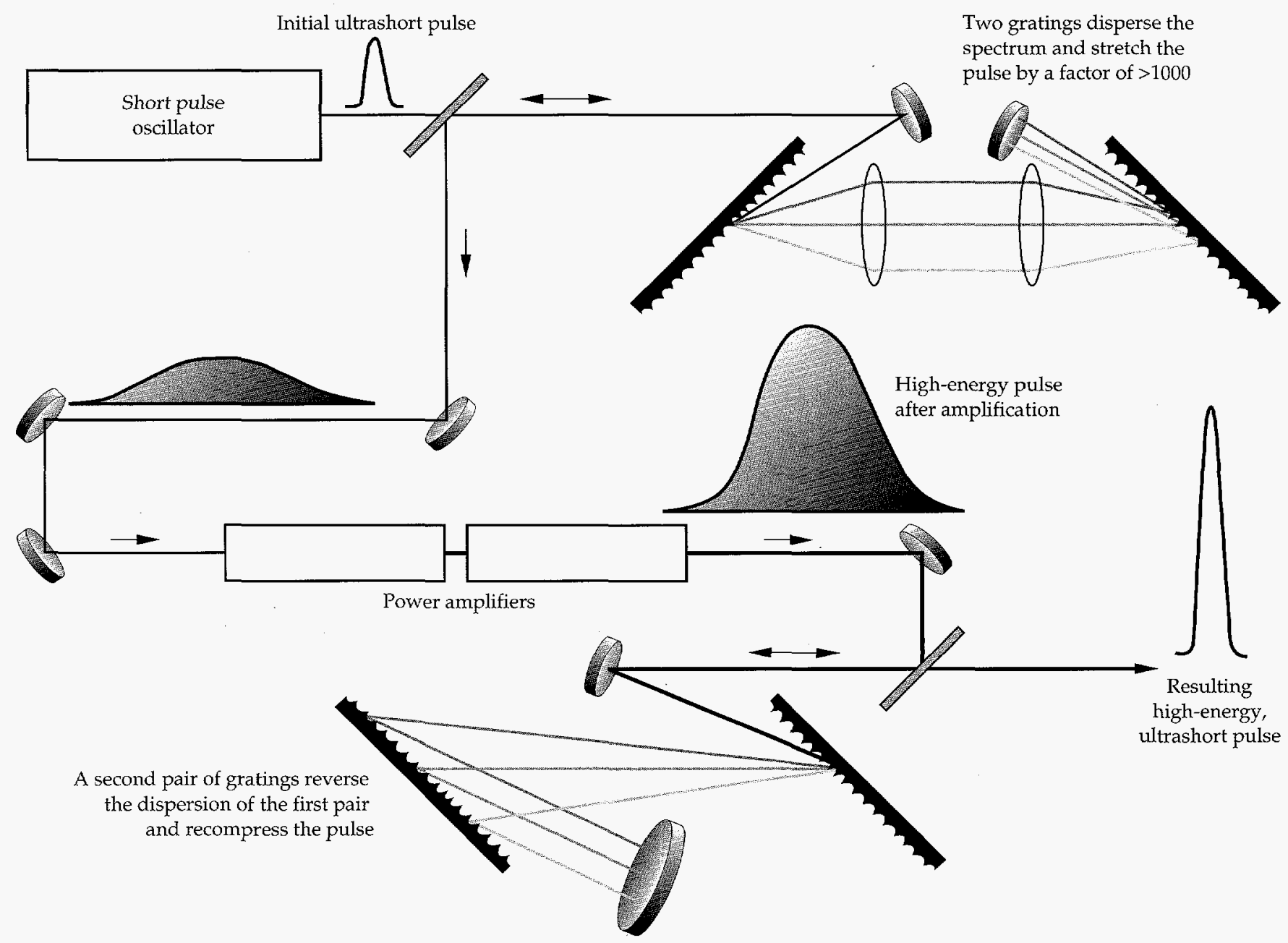

FIGURE 1. Partial schematic of beam stretching, amplifying, and compressing system used in high-power chirped pulse amplification (CPA), showing location of gratings and their function-giving different beams longer or shorter paths to stretch or compress the pulse. 
a damage threshold above $40 \mathrm{~J} / \mathrm{cm}^{2}$ for 1-ns pulses and above $2 \mathrm{~J} / \mathrm{cm}^{2}$ for 400 -fs pulses. ${ }^{14}$ Dielectric transmission gratings exhibiting high diffraction efficiency have been produced. ${ }^{15}$ Unfortunately, the efficiency of these gratings in reflection is poor. In this article, we describe the design and performance of multilayer dielectric gratings that can achieve up to $98 \%$ diffraction efficiency in reflection in the $m=-1$ order, and that exhibit damage thresholds higher than those achievable with metallic gratings.

\section{Basic Grating Considerations}

The basic geometric properties of any planar grating follow from the grating equation, which expresses the condition for constructive interference from successive periodic elements on a surface and which relates the incident angle $\theta_{\mathrm{i}}$, the diffracted angle $\theta_{\mathrm{m}}$ for order $m$, and the ratio of wavelength $\lambda$ to groove spacing $d$ :

$\sin \theta_{m}=\sin \theta_{i}+m \lambda / d$

Although these few parameters control the possible presence and direction of various diffracted orders, the distribution of energy among the orders (quantified by the grating efficiency) is determined by the wavelength and polarization of the incident light, the depth and shape of the grooves, and the optical properties of the diffracting structure. The groove profile depends on the method of manufacture and differs between ruled gratings (triangular profiles), holographic gratings (typically sinusoidal profiles) and etched lamellar gratings (rectangular or fin-shaped profiles).

For a reflection grating used in a first-order Littrow mount, the angle of incidence is fixed by the condition $\sin \theta_{i}=\lambda / 2 d$. When $\lambda / d>2$, only specular reflection $(m=0)$ and evanescent orders occur; for $2>\lambda / d>2 / 3$, two propagating orders occur (specular reflection, $m=0$, and retrodiffraction, $m=-1$ ). This latter two-order regime accounts for nearly all high-power laser applications.

Our grating designs are based on a rigorous solution of Maxwell's equations (with appropriate boundary conditions) for diffraction from a multilayer structure with a periodic surface-relief profile as shown in Fig. 2. We idealize the radiation as a monochromatic plane wave, linearly polarized as either transverse electric, TE (electric field along the grooves), or transverse magnetic, TM (magnetic field along the grooves). The grating profile can be of arbitrary shape, and the dielectric layers are specified by the layer thickness and an index of refraction. Our computations use the multilayer modal method. ${ }^{16-19}$ This method replaces the corrugated grating surface by a succession of slices (rectangular in cross section), in each of which the complex-valued dielectric constant alternates periodically between two values, corresponding to the materials above and below the grating surface. Exact normal-mode solutions to the vector Helmholtz equation are found within the slices (an eigenvalue problem), and boundary conditions are matched in moment form to produce a complete solution. Solutions are carried between layers using an R-matrix method. The mathematical methods employed are discussed in detail elsewhere. ${ }^{12,18,19}$

Figure 3 presents the predicted $m=-1$ diffraction efficiency of a 1550 -groove $/ \mathrm{mm}$ grating used at the Littrow angle $\left(56^{\circ}\right)$ for TE-polarized 1053-nm light incident on the multilayer structure of Fig. 2. The multilayer is composed of alternating layers of high-index $\mathrm{ZnS}(n=2.35)$ and low-index $\operatorname{ThF}_{4}(n=1.52)$ to form a highly reflecting quarter-wave stack. The top layer, containing the grating, is composed of the high-index $(\mathrm{ZnS})$ layer. High diffraction efficiency is achieved by controlling the thickness $t$ of the top layer and the depth $h$ of the trapezoidal grooves. The maximum diffraction efficiency (predicted to be above 98\%) occurs for an infinite periodic series of choices for $t$. To simplify fabrication, we always choose the thinnest solution.
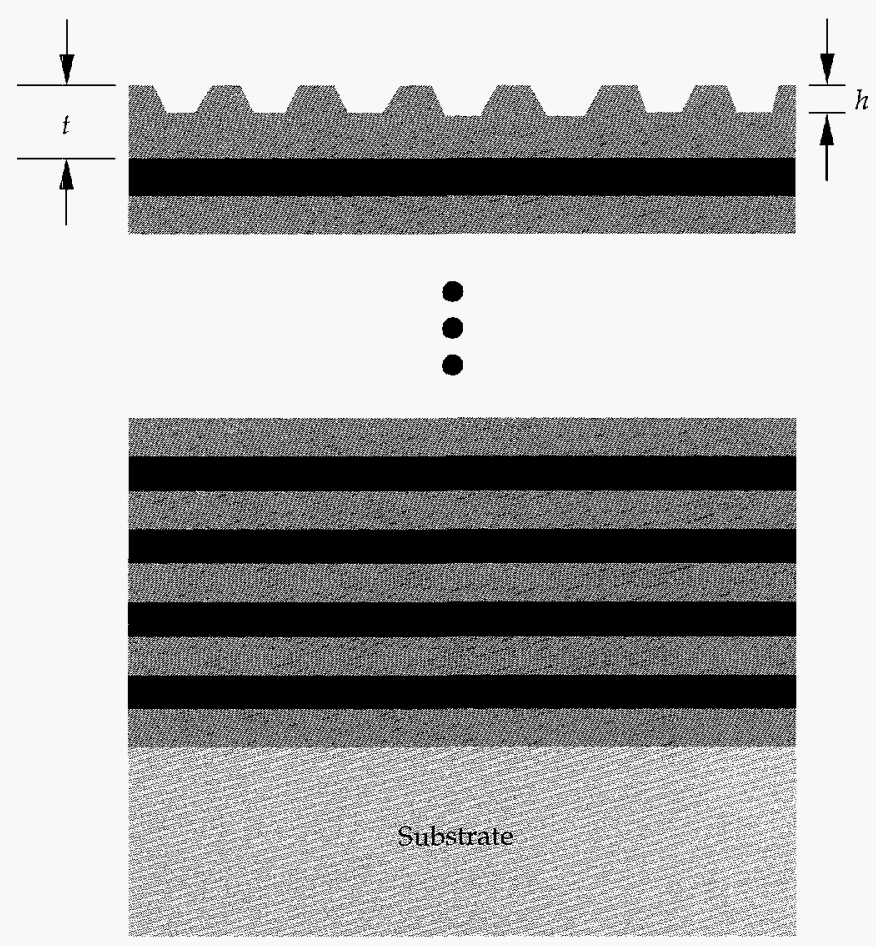

FIGURE 2. Basic multilayer dielectric grating concept $(h=$ groove depth, $t=$ top layer thickness). Dark and light layers represent materials of high $(n \approx 2)$ and low $(n \approx 1.5)$ refractive index, respectively. (70-60-0695-1667pb01) 
Figure 3 gives a detailed view of the diffraction efficiency as a function of $h$ and $t$. Strong diffraction occurs when the optical depth of the grooves is near one quarter of a wavelength and the optical thickness of the top layer is near three quarters of a wavelength. Our calculations suggest high sensitivity to the incident polarization, as might be expected from the polarization sensitivity of conventional multilayer coatings. The peak diffraction efficiency for TM (p-polarized) light on this structure is predicted to be less than $50 \%$; that for TE (s-polarized) light is predicted to be near $98 \%$.

\section{Manufacturing Procedure}

Our gratings are fabricated using lithographic techniques following holographic exposure. The dielectric multilayer structure is vacuum-deposited by e-beam evaporation on an optically flat (better than $\lambda / 12$ ) substrate. The substrate is then coated with a thin $(\sim 300 \mathrm{~nm})$ film of photoresist (Shipley 1400) and cured at $80^{\circ} \mathrm{C}$ for
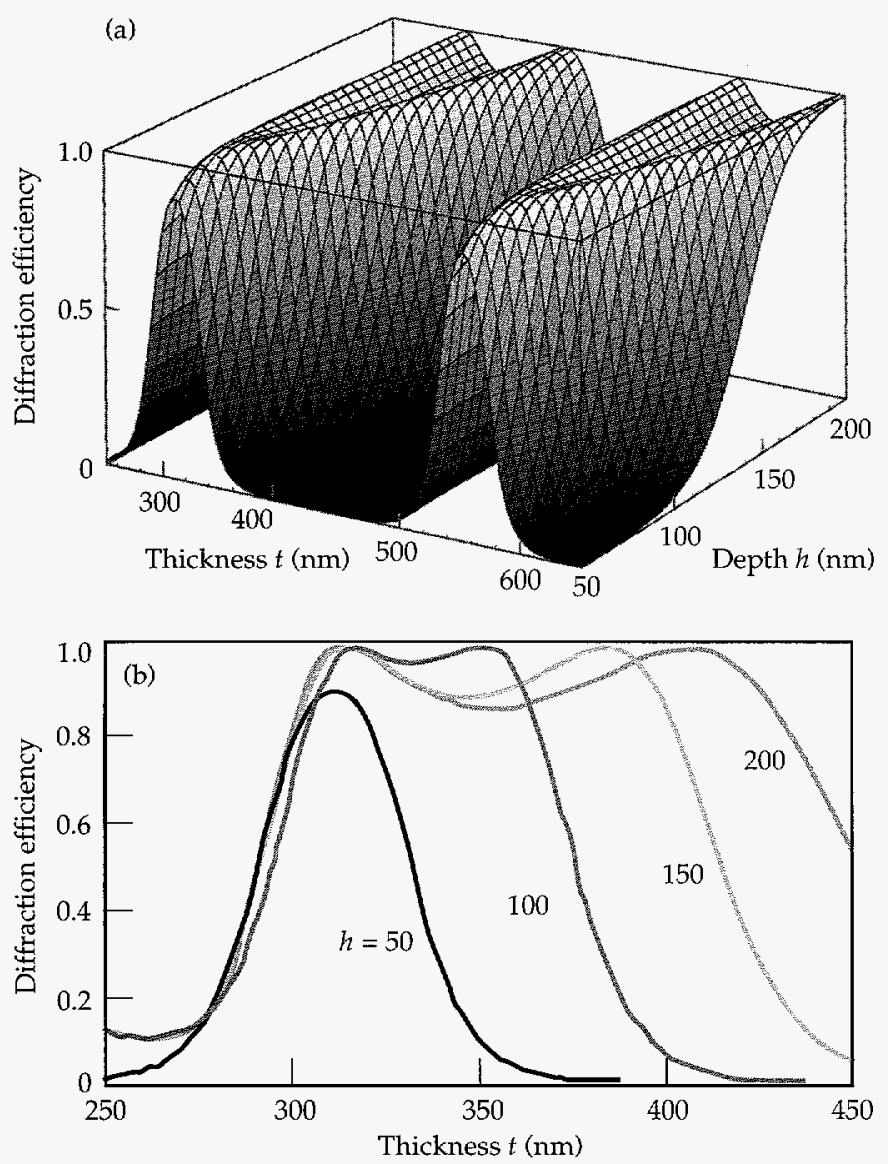

FIGURE 3. (a) Theoretical diffraction efficiency (order $m=-1$ ) for TE-polarized light at $1053 \mathrm{~nm}$ for a multilayer dielectric grating consisting of alternating layers of $\mathrm{ZnS}$ and $\mathrm{ThF}_{4}$. Trapezoidal grooves are etched into the top layer as illustrated in Fig. 2. (b) Expanded view of first maxima of efficiency surface, showing efficiency (order $m=-1$ ) for depths $h=50,100,150$ and $200 \mathrm{~nm}$. (70-60-0695-1668pb01)
$30 \mathrm{~min}$. The surface relief pattern spacing $d$ is produced in the photoresist by intersecting two laser beams, each of exposure wavelength $\lambda_{\mathrm{e}}$ and each incident at an angle $\theta_{\mathrm{e}}$ according to the formula

$d=\frac{\lambda_{e}}{2 \sin \theta_{e} \cos \phi}$,

where $\phi$ is the angle between the normal to the substrate and the bisector of the incident laser beams (see Fig. 4). The interference pattern was produced by an equal-path, fringe-stabilized interferometer utilizing a 2-W single-longitudinal-mode $\mathrm{Kr}$-ion laser (Coherent) operating at $413 \mathrm{~nm}$. Straight, parallel grooves are produced only by highly collimated radiation; even the slightest wavefront curvature of the interfering beams produces curved grooves with nonuniform spacing. This groove distortion reduces the spectral resolution of the grating and produces undesirable curvature in the wavefront of the diffracted beam.

Once the interference pattern is recorded in the photoresist, development removes those regions exposed to the laser light (positive resist), producing a corrugated surface relief profile that is transferred into the substrate material by reactive-ion etching. ${ }^{19}$ Although the interference fringe pattern exhibits a sinusoidal intensity distribution, the groove shape ultimately produced in the grating is affected by a number of process variables. Our most common profile is trapezoidal, so we optimize the designs for trapezoidal grooves.

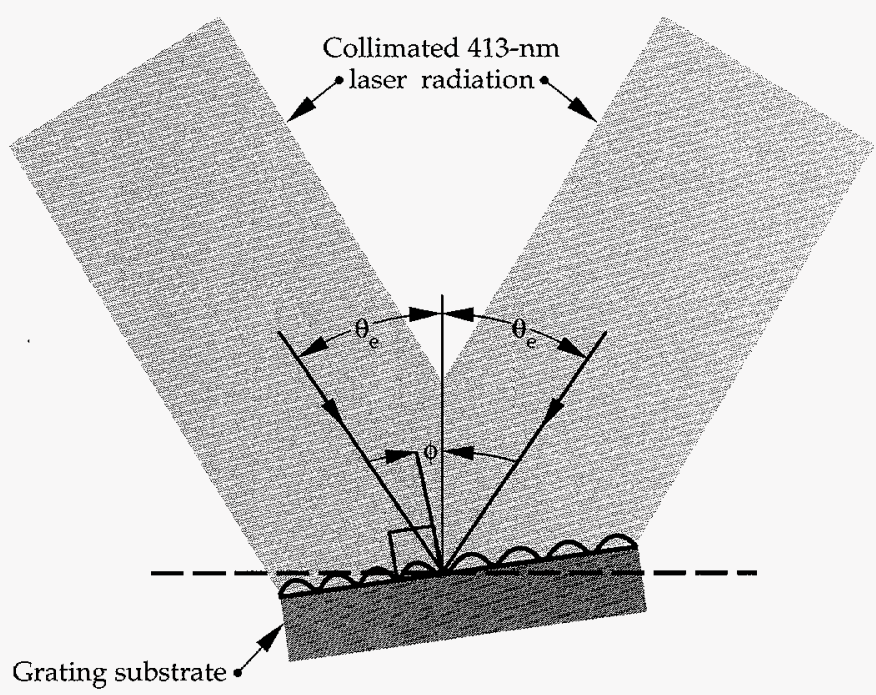

FIGURE 4. Holographic exposure geometry, showing definition of angle of incidence $\theta$ and angle $\phi$ between grating normal and bisector of incident waves. (70-60-0695-1669pb01) 
Figure 5 shows a typical scanning electron micrograph of a cross section of a completed grating.

We determined the dependence of the diffraction efficiency of this grating on the angle of incidence by measuring the average power of the diffracted $(m=-1$, near the Littrow angle), reflected $(m=0)$, and incident beams produced by a narrow-linewidth Ti:sapphire $\mathrm{cw}$ laser operating at $1053 \mathrm{~nm}$. Figure 6 shows the results of this measurement. The difference between the incident beam and the sum of the diffracted and reflected beams arises from a net scattering of $\sim 1 \%$ and a transmission loss of $\sim 0.5 \%$. Low diffraction efficiency is observed until the design angle of $56^{\circ}$ is approached; there the reflected $(m=0)$ energy for TE polarization drops to approximately $1 \%$ of the incident energy and the $m=-1$ diffracted beam reaches a peak efficiency of 96.1\%. For TM polarization, the maximum diffraction efficiency is $50 \%$, in good agreement with the predicted value. We observe a small variation of efficiency (from a high of $96 \%$ to a low of $94 \%$ ) over the surface of our gratings.

The measured damage threshold of our oxide $\left(\mathrm{HfO}_{2} / \mathrm{SiO}_{2}\right.$ )-based multilayer dielectric gratings for 1-ns laser pulses is over $5 \mathrm{~J} / \mathrm{cm}^{2}$, nearly ten times that of the best metallic gratings. For pulse durations from 0.3 to 5 ps, our gratings exhibit a damage threshold of

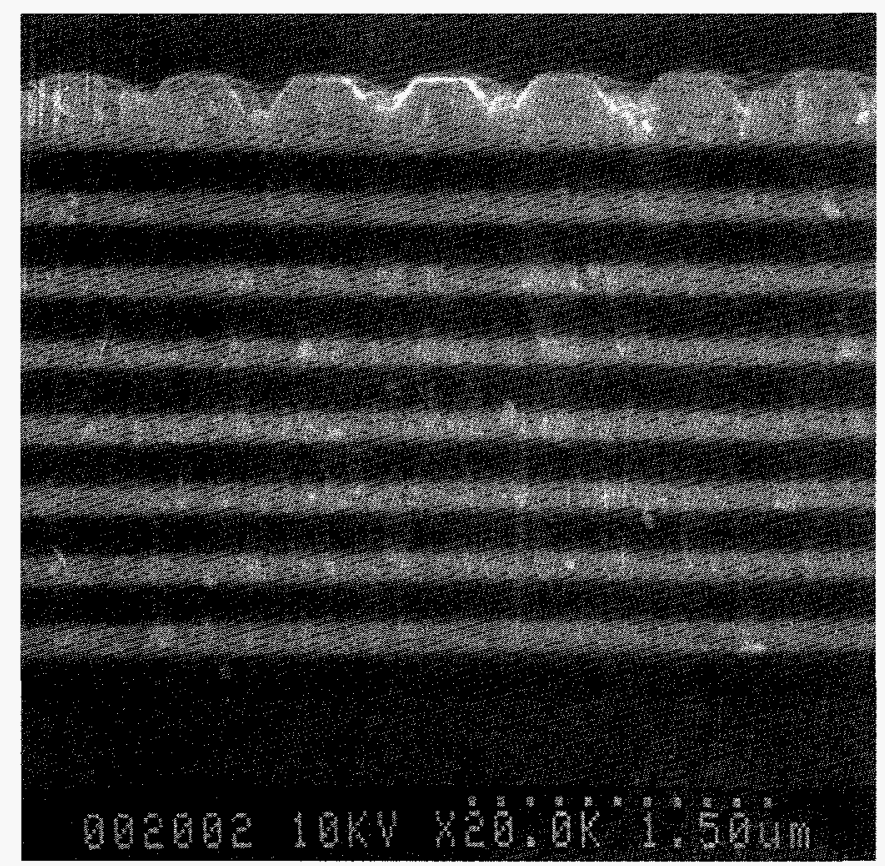

FIGURE 5. Scanning electron micrograph of multilayer dielectric grating structure. $\quad(20-03-0795-1855 \mathrm{pb} 01)$ approximately $0.6 \mathrm{~J} / \mathrm{cm}^{2}$, three times higher than the short-pulse damage threshold of commercial metallic gratings. Further refinement of our multilayer design is expected to increase the short-pulse damage threshold to over $1 \mathrm{~J} / \mathrm{cm}^{2}$.

In addition to the requirement of high diffraction efficiency and high damage threshold, gratings used for pulse compression must maintain high efficiency over a large bandwidth. Figure 7 shows the calculated

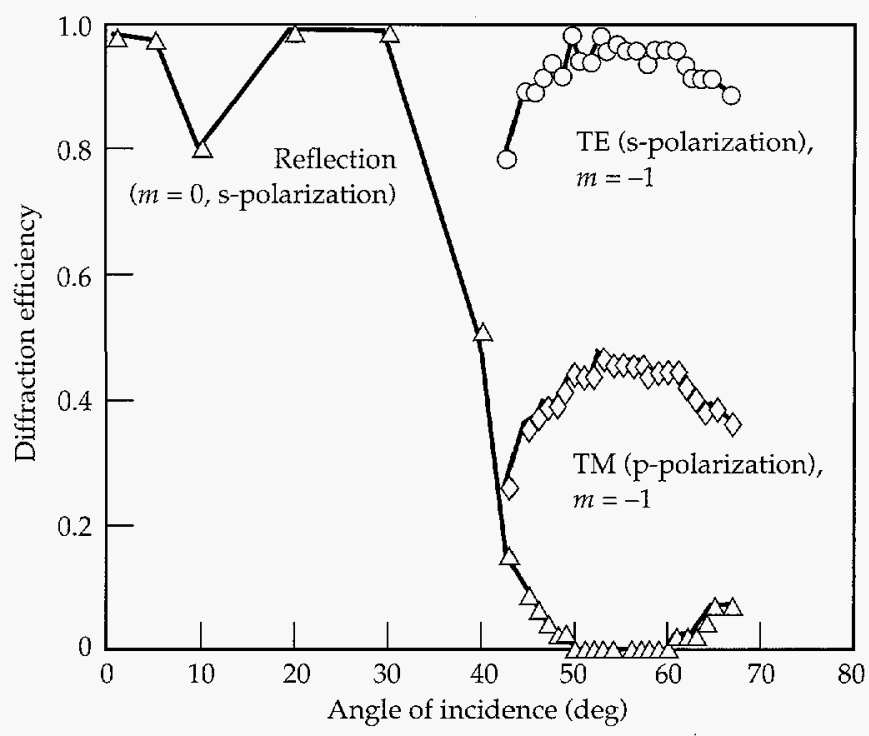

FIGURE 6. Absolute diffraction efficiency (order $m=-1$, Littrow mount) at a wavelength of $1053 \mathrm{~nm}$ and for various polarizations for the grating shown in Fig. 5. (70-60-0695-1671pb01)

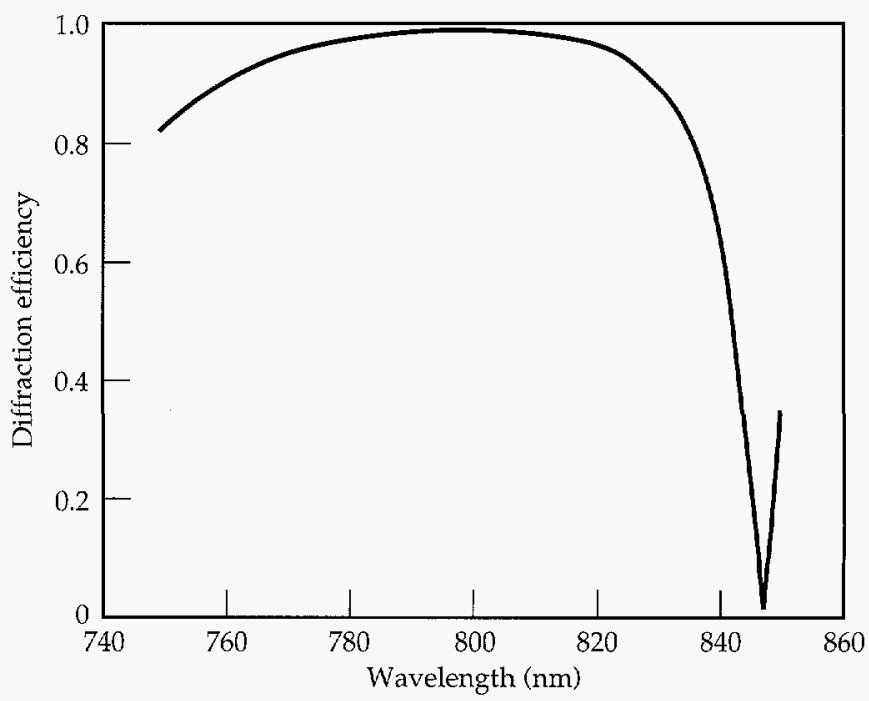

FIGURE 7. Theoretical diffraction efficiency $(m=-1)$ vs wavelength of an oxide-based multilayer grating ( 1800 grooves $/ \mathrm{mm}$ ). Bandwidth is over $85 \mathrm{~nm} . \quad(70-60-0695-1672 \mathrm{pb01})$ 
wavelength dependence of the diffraction efficiency of diffraction efficiency an oxide-based multilayer grating (8 layer pairs) optimized for use with 100 -fs pulses at $800 \mathrm{~nm}$. Diffraction efficiency above $90 \%$ is maintained from 760 to over $830 \mathrm{~nm}$. Such gratings could easily handle pulses as short as $20 \mathrm{fs}$. We can increase the bandwidth, at the price of a slightly reduced peak diffraction efficiency, by reducing the number of layers or changing the design slightly. We have produced designs that can support pulses as short as $10 \mathrm{fs}$ with greater than $90 \%$ diffraction efficiency. At these extremely short pulse durations, the dispersion of the multilayer coating itself will become important. We have not yet examined this effect on our ability to use these gratings for stretching and compressing 10-fs pulses.

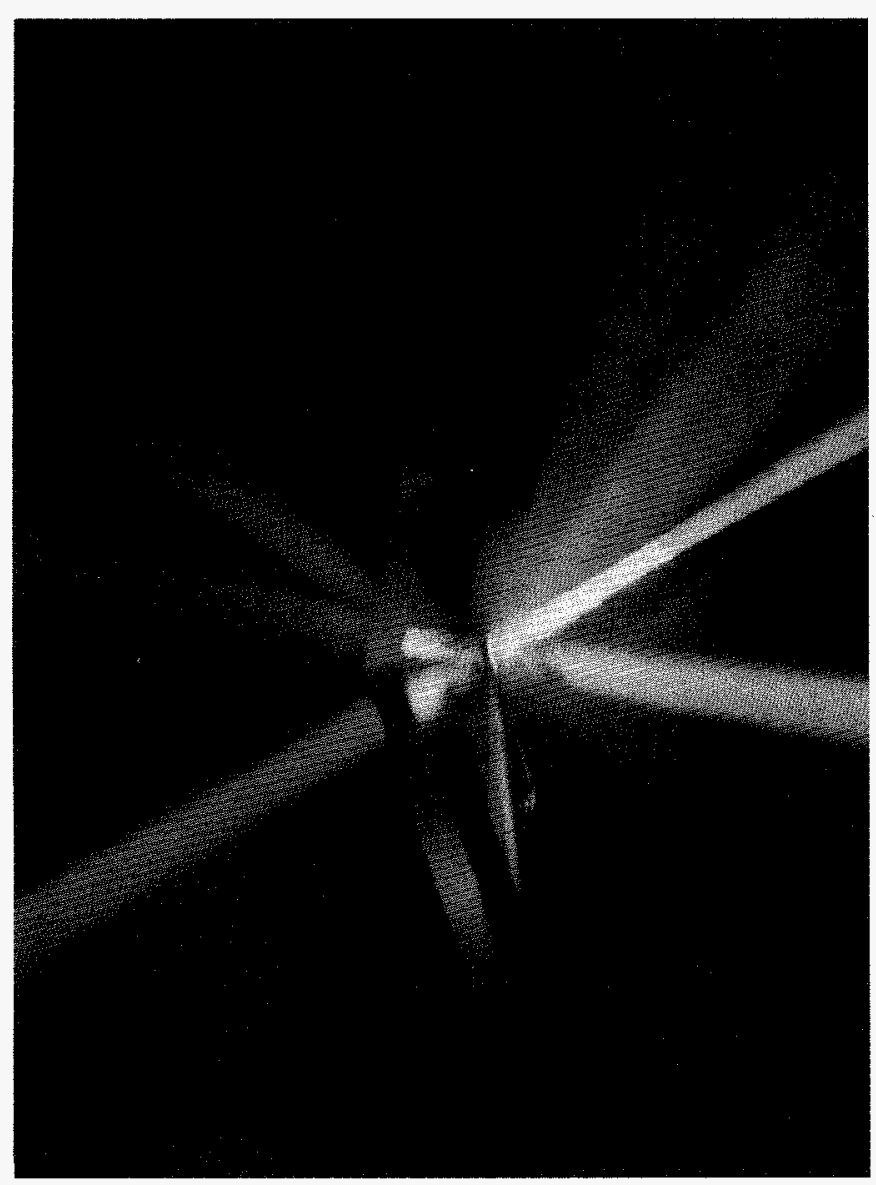

FIGURE 8. Multilayer dielectric diffraction grating designed to reflect yellow light, diffract broadband visible radiation (bottom left), eliminate all green and yellow light in the transmitted diffracted beam (right), and transmit blue-green light. The grating pictured is $15 \times 20 \mathrm{~cm}$. (70-15-0294-0178Apb01)

\section{Applications of Multilayer Dielectric Gratings}

Another feature of this new type of diffraction grating is the ability to design the grating to perform a multitude of functions simultaneously. Specifically, the gratings can be designed as beamsplitters that transmit, diffract, and reflect light. By controlling the design of the multilayer and the grating, specific wavelengths can be transmitted, others reflected, and still others diffracted, all with specified efficiency. As an example, we fabricated a grating that operated simultaneously as a broadband diffraction grating in reflection $(10 \%$ efficiency in the $m=-1$ order from 390 to $700 \mathrm{~nm}$ ), a high reflector in the yellow (90\% reflection from $\sim 570$ to $590 \mathrm{~nm}$ ), a high transmitter in the blue-green $(\sim 90 \%$ from 500 to $570 \mathrm{~nm}$ ), and a notch filter for the transmitted diffracted $(m=-1)$ beam [extremely low efficiency $(<1 \%)$ for 500 to $600 \mathrm{~nm}$ and relatively high efficiency $(>50 \%)$ for 400 to $500 \mathrm{~nm}$ and 600 to $700 \mathrm{~nm}$ ). Figure 8 displays this performance.

\section{Summary}

We have demonstrated techniques for designing and holographically creating diffraction gratings, based on a multilayer dielectric structure, that exhibit diffraction efficiency exceeding $96 \%$ into the $m=-1$ order in a near-Littrow configuration. The high diffraction efficiency is obtained by proper coating design and by adjustment of the depth of the grooves and the thickness of the top layer. By adjusting the coating design, gratings of essentially any efficiency and variable bandwidth can be produced.

The damage thresholds of these all-dielectric gratings surpass those of metal gratings. To date, relative to commercial metallic diffraction gratings, our dielectric gratings have achieved an order-of-magnitude increase in damage threshold for nanosecond pulses and a factor-of-three increase for subpicosecond pulses.

\section{Acknowledgments}

We thank E. Lindsay and C. Moore for producing the electron and atomic-force micrographs and C. Hoaglan for his technical assistance. We are indebted to Lifeng $\mathrm{Li}$, of the University of Arizona, for ongoing advice and assistance. 


\section{Notes and References}

1. M. Tabak, J. M. Hammer, M. E. Glinsky, W. L. Kruer, et al., ICF Quarterly Report 4(3);90-100, Lawrence Livermore National Laboratory, Livermore, CA, UCRL-LR-105821-94-3 (1994).

2. P. Maine, D. Strickland, P. Bado, M. Pessot, and G. Mourou, IEEE I. Quantum Electron. QE-24, 398-402 (1988).

3. J. Kmetec, J. J. Macklin, and J. F. Young, Opt. Lett. 16, 1001-3 (1991).

4. A. Sullivan, H. Hamster, H. C. Kapteyn, S. Gordon, Opt. Lett. 16, 1406-8 (1991).

5. M. Pessot, J. Squier, G. Mourou, and D. Harter, Opt. Lett. 14, 797-799 (1989); M. Pessot, J. Squier, P. Bado, G. Mourou, and D. Harter, IEEE J. Quantum Electron. QE-25, 61-66 (1989).

6. T. Ditmire and M. D. Perry, Opt. Lett. 18, 426-428 (1993); T. Ditmire, H. Nguyen, and M. D. Perry, J. Opt. Soc. Amer. B 11, 580 (1993).

7. P. Beaud, M. Richardson, E. J. Miesak, and B. H. T. Chai, Opt. Lett. 18, 1550-52 (1993).

8. M. D. Perry and R. Olson, Laser Focus World 27, 69 (1991).

9. C. Rouyer, E. Mazataud, I. Allais, A. Pierre, et al., Opt. Lett. 18, 214-216 (1993).

10. E. B. Treacy, IEEE J. Quantum Electron. QE-5, 454-8 (1969).
11. M. C. Hutley, Diffraction Gratings (Academic, NY, 1982).

12. R. Boyd, J. Britten, D. Decker, B. W. Shore, et al., Appl. Opt. 34, 1697 (1995)

13. E. Popov, L. Tsonev, and D. Maystre, J. Mod. Opt. 37, 367-377 (1990).

14. B. C. Stuart, M. D. Perry, and B. W. Shore, "PulsewidthDependent Damage Measurements of Dielectric Materials," Lawrence Livermore National Laboratory, Livermore, CA, UCRL-JC-118115. Prepared for proceedings of Physics with Intense Laser Pulses, St. Malo, France, August 1994.

15. J. J. Armstrong, Holographic Generation of Ultra-High-Efficiency Large-Aperture Transmission Diffraction Gratings (Ph.D. Thesis, University of Rochester, 1993).

16. R. Petit, Ed. Electromagnetic Theory of Gratings (Springer, Berlin, 1980).

17. D. Maystre, "Rigorous Vector Theories of Diffraction Gratings," in Progress in Optics, E. Wolf, Ed. (Elsevier, NY, 1984), vol. 21, pp. 1-67.

18. L. Li, J. Opt. Soc. Am. A 10, 2581-91 (1993); L. Li, J. Mod. Opt. 40, 553-73 (1993); L. Li, J. Opt. Soc. Am. A 11, 2816-2828 (1994).

19. L. Li and C. W. Haggans, J. Opt. Soc. Am. A 10, 1184-1189 (1993). 


\title{
EXPERIMENTAl Characterization of HohlRaum CONDITIONS BY X-RAY SPECTROSCOPY
}

\author{
C. A. Back \\ B. F. Lasinski \\ L. J. Suter \\ E. J.Hsieh \\ B. J. MacGowan \\ G. F. Stone \\ R. L. Kauffman \\ L. V. Powers \\ R. E. Turner \\ T. D. Shepard
}

\section{Introduction}

Spectroscopy is a powerful technique used to measure the plasma parameters relevant to Inertial Confinement Fusion (ICF) plasmas. For instance, the onset of spectral signals from multilayer targets have been used to determine ablation rate scalings. ${ }^{1}$ Temperature and density measurements in coronal plasmas have enabled the study of laser coupling efficiency as a function of the laser wavelength. ${ }^{2}$ More recently, dopants have been successfully used to determine capsule conditions of ICF targets. ${ }^{3}$ However, few spectroscopic studies have been performed to diagnose plasma conditions of the hohlraum itself. Several laboratories have studied enclosed cavities, ${ }^{4}$ previously concentrating on measurements of the radiative heat wave, the x-ray conversion efficiency, and temporal evolution of Au $x$ rays.

Measurements of electron temperature $T_{e}$ and electron densities $n_{\mathrm{e}}$ are difficult because many physical processes occur and each diagnostic's line-of-sight is restricted by the hohlraum wall. However, they are worth pursuing because they can provide critical information on the target energetics and the evolution of plasma parameters important to achieving fusion.

In this article, we discuss spectroscopic tracers to diagnose plasma conditions in the hohlraum, using time- and space-resolved measurements. The tracers are typically mid- $Z$ elements $(Z=13-24)$, which are placed on the hohlraum wall or suspended in the hohlraum volume. To demonstrate the breadth of measurements that can be performed, three types of experiments are presented. The first set tests ablation inside hohlraums by using tracers under the laser beam focal spot. The second set examines the heating of the wall by the tracers buried at different depths. The third set measures $T_{\mathrm{e}}$ by analysis of line intensity ratios. Spectroscopy has an advantage over imaging studies because it can provide nonperturbative measurements of local plasma parameters, i.e., $T_{\mathrm{e}}$ and $n_{\mathrm{e}}$. Furthermore, not only can a spectra allow a measurement of plasma conditions, but its temporal behavior can reveal information about the hydrodynamics and heating of the hohlraum.

\section{Experimental Approach}

Microdots aid the spectroscopic analysis by limiting optical depth, localizing the tracer, and reducing edge effects of laser irradiation. ${ }^{5}$ Target fabrication techniques successfully produce versatile hohlraums having tracer dopants deposited as high-precision microdots or strips. The tracers are deposited on a thin substrate such as an $800-\AA \mathrm{CH}$ foil or on a $25-\mu \mathrm{m}$-thick Au foil that mimics the hohlraum wall. The advantages of using tracers for probing the plasma include: (1) creating a localized plasma to track the dopant by emission or absorption, (2) controlling the thickness of the dopant, (3) systematically varying the depth of the tracer to probe different layers below the surface, and (4) defining the transverse plasma length probed to produce optically thin transitions. These advantages are important to successfully diagnose the complex hohlraum environment. LASNEX simulations of plasmas created from tracers indicate that the plasma can remain localized inside the hohlraum. For instance, different positions of the density gradient can be probed by suitably choosing the depth at which a tracer is buried. ${ }^{6}$ Thus the experiments can be particularly effective in understanding ablation, plasma formation in nonplanar geometries, and overall energy balance. 
Since the plasmas may be $>1 \mathrm{~mm}$, the accuracy of spectroscopic measurements have benefited from the development of $x$-ray streak cameras and gated imagers. Streak cameras provide continuous temporal resolution of the spectral signal while gated imagers allow imaging of the spectra produced from a microdot or strip. Recently, we developed and. fielded a spectrograph using a Bragg x-ray diffraction crystal coupled to a gated module, ${ }^{7}$ which converts one dimension of the spatial resolution to spectral resolution. Its primary advantage over other diagnostics is that it spectrally images $\mathrm{x}$-ray emission from laser-produced targets in 250 ps time frames. These technological developments have been essential to achieve sufficient spatial and spectral resolution of these targets.

\section{Experimental Results}

Figure 1 is a schematic of the target showing the placement of tracer foils. Tracers are placed on the wall for ablation and wall heating experiments, as indicated in the figure. Tracers are deposited on a freestanding foil that is suspended inside the hohlraum for recent experiments measuring the $T_{\mathrm{e}}$ of gas-filled targets, also shown in the figure. This section discusses three types of experiments that can be performed: ablation measurements, embedded microdot emission measurements, and $T_{\mathrm{e}}$ measurements.

\section{Ablation Measurements}

In ablation experiments, we compared a lined and unlined hohlraum by monitoring the emission of $\mathrm{KCl}$

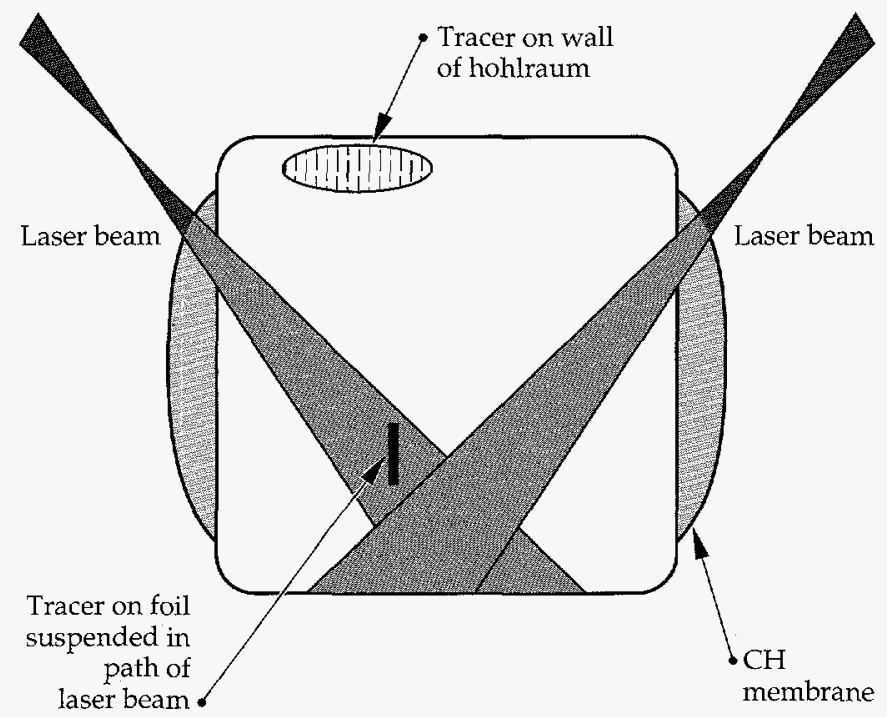

FIGURE 1. Schematic of a hohlraum target showing the position of patches and suspended foils. (08-20-0695-1660pb01) that was deposited on a Au foil on the hohlraum's inside surface. The $\mathrm{KCl}$ was directly irradiated by a laser beam and observed with $\mathrm{x}$-ray streak cameras. The dot provided spectral signals that served as a temporal marker of the laser incident on the hohlraum's inside surface.

The targets were scale- 1 hohlraums that were either lined with $7500 \AA$ of $\mathrm{CH}$ or unlined. At the position of beamline 6 , a hole was drilled into the hohlraum and covered with a foil patch with the $\mathrm{KCl}$ facing the inside of the hohlraum. The patch consisted of a $25-\mu \mathrm{m} \mathrm{Au}$ foil overcoated with $3500 \AA$ of $\mathrm{KCl}$. Eight Nova beams at $3 \omega_{\mathrm{o}}$ irradiated the target in the standard pointing and focusing geometry to produce focal spots on the hohlraum wall $\sim 550 \mu \mathrm{m} \times 900 \mu \mathrm{m}$ diam. The total laser energy was $22.3 \mathrm{~kJ}$ with $<5 \%$ variation. In a $1 \mathrm{~ns}$ square pulse, this produces an intensity of $6 \times 10^{14} \mathrm{~W} / \mathrm{cm}^{2}$ in each focal spot.

Figure 2 shows an example of the data. Line intensity traces as a function of time were taken for the $\mathrm{He}$ like $\alpha$ line $(n=2-1)$ of $\mathrm{Cl}, 1 \mathrm{~s}^{2}\left({ }^{1} \mathrm{~S}_{0}\right)-1 \mathrm{~s} 2 \mathrm{p}\left({ }^{1} \mathrm{P}_{1}\right)$. For comparison, the Au emission was taken as close as possible to the short wavelength side of the line to minimize differences in detector response and to avoid the $\mathrm{Cl}$ satellites.
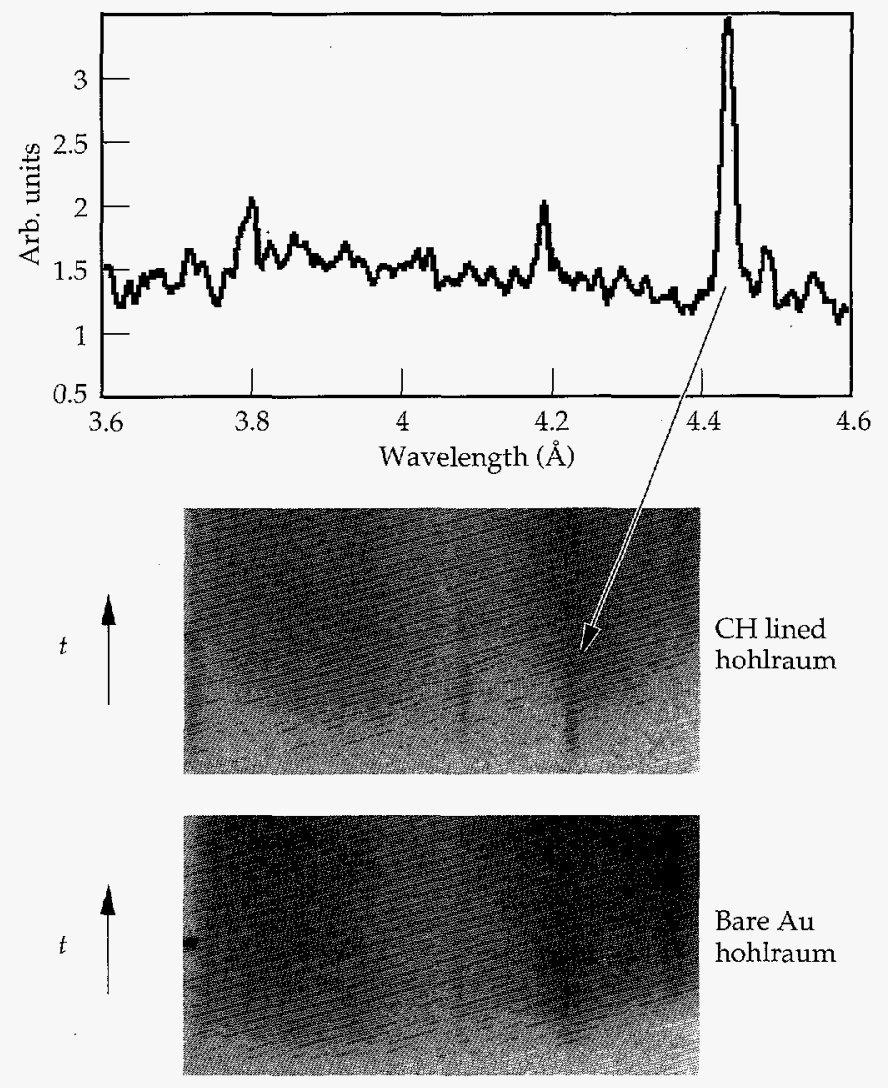

FIGURE 2. Example of data and a lineout from ablation experiments. (08-20-0695-1661pb01) 
Figure 3 compares these four traces. There are two salient features of the temporal evolution. Early in time, the slope of the emission can be approximated by a straight line for all the lineouts. Later in time, the slope changes abruptly for all the cases except the Au emission from the lined hohlraum.

We define the burnthrough time as the temporal delay between the $\mathrm{Cl}$ emission from the tracer and $\mathrm{Au}$ emission from the lined or unlined hohlraum. In the unlined case, the $\mathrm{KCl}$ burns through and Au emission is detected 40 ps later when comparing the half maximum intensity. The lined case shows burnthrough to the Au 100 ps later. The onset of the signal taken is $t=0$, where we assume that the delay in ionizing to He-like $\mathrm{Cl}$ is negligible. Here, the relative difference in the $\mathrm{Au}$ vs $\mathrm{Cl}$ emission is more important than the absolute value of the timing. The reference intensity for each case is the intensity when the $\mathrm{KCl}$ signal is no longer distinct from the Au signal. The difference reveals that there is a lag of $60 \mathrm{ps}$ between the lined and unlined case.

A noticeable shoulder in the data exists where the slope of the intensity changes. Initially, the $\mathrm{KCl}$ in both the unlined and lined case follow the same curve, then their evolutions differ. If we consider the $\mathrm{KCl}$ as only a timing marker, the delay in burnthrough, as determined from the relative time lag of Au emission, is $80 \mathrm{ps}$. This delay has physical significance since the $\mathrm{KCl}$ foil was not overcoated with $7500 \AA$ of $\mathrm{CH}$ and is therefore exactly the same in the two cases.

Because the intensity of the $\mathrm{Cl}$ emission is identical for the first 40 ps of emission, it is consistent to interpret the departure of the $\mathrm{Cl}$ slope as the inability of the laser to continue to heat the $\mathrm{KCl}$ in the $\mathrm{CH}$ lined hohlraum in the same way as the $\mathrm{KCl}$ in the unlined hohlraum. In addition, the intensity of the unlined and lined cases are different by a factor of two after 600 ps. Although the unlined and lined data are from two different experiments, the shots were performed on the

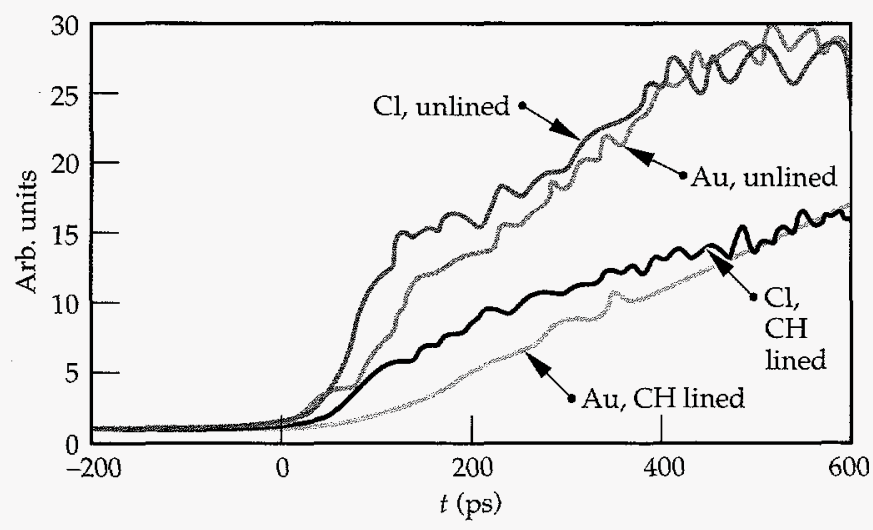

FrgURE 3. Comparison of the emission from Fig. 2. (08-20-0695-1662pb01) same day, the target setup and spectrometer were identical, and differences due to film processing are minimal. Ablated $\mathrm{CH}$ inside of the hohlraum would produce a negligible attenuation of the signal. Therefore, the difference in intensity would indicate that less emission is detected in the lined hohlraum from both $\mathrm{Au}$ and $\mathrm{Cl}$ than from the unlined hohlraum.

Another measure of the target ablation is the time at which the $\mathrm{KCl}$ emission is no longer distinct from the Au emission. For the lined hohlraums, a detectable $\mathrm{KCl}$ signal persists for a longer period of time, $470 \mathrm{ps}$ vs $280 \mathrm{ps}$. The duration of the $\mathrm{KCl}$ signal can be correlated with the amount of time that the $\mathrm{KCl}$ is localized enough to produce a signal above the Au background. When the $\mathrm{KCl}$ is no longer discernible from the $\mathrm{Au}$, the total signal of the lined hohlraum is $70 \%$ of the unlined signal. When the $\mathrm{KCl}$ contribution is subtracted from the Au signal, the peak intensity of the lined hohlraum is $85 \%$ of the unlined signal. The result implies that the $\mathrm{CH}$ lined hohlraum has a slower ablation rate and does not cause Au emission of equal intensity within the first 600 ps of the laser pulse.

The measure of the burnthrough rate shows a measurable delay in time corresponding to $\sim 80$ ps. However, intensity of the Au emission is markedly different in both magnitude and evolution. Clearly at 600 ps, the Au has roughly half the integrated intensity, and the projection of the slope of the intensity vs time indicates that even after $1 \mathrm{~ns}$, the Au emission of the lined hohlraum may not reach that of the unlined hohlraum.

\section{Embedded Microdot Hohlraum Experiments}

Embedded microdot hohlraum experiments employed tracers buried under $\mathrm{CH}$ at a depth of 1 to $3 \mu \mathrm{m}$. The tracers were $250-\mu \mathrm{m}$-diam microdots of cosputtered $\mathrm{Ti}$ and $\mathrm{Cr}$ placed under a laser focal spot in a 3.2-mm-long hohlraum. Analysis of the spectra allowed us to examine heating of the inside hohlraum wall under a laser focal spot. Figure 4 shows an example of a TiCr K-shell spectrum where there is no appreciable $\mathrm{H}$-like $\mathrm{Cr}$. The top graph of Fig. 4 shows the laser pulse used to heat the hohlraum. The emission becomes detectable by a gated spectroscopic imager only $\sim 2.2 \mathrm{~ns}$ after the beginning of the shaped pulse. Contrary to expectations, the tracer plasma does not become more ionized as the laser intensity increases to its peak. Calculations from foil burnthrough targets predict an $T_{\mathrm{e}}$ of $\sim 2.5 \mathrm{keV}$. Due to the absence of $\mathrm{H}$-like $\mathrm{Cr}$ in the experimental data, we conclude that the plasma remains less ionized than expected during the laser pulse. Based on the intensity of the $\mathrm{He}$-like $\beta$ resonance lines $(n=3-1)$ of $\mathrm{Ti}$ and $\mathrm{Cr}$, the temperature derived from the isoelectronic sequence ratio is $<2 \mathrm{keV}$. The spectra are $5 \%$ wider than the experimental width, 
which indicates that line broadening or source motion may also be occurring. The late time spectrum shows the return of the $\mathrm{Cr}$ He-like $\beta$ line, which probably indicates that recombination is occurring. Because the tracer plasma was cooler than expected and the $\mathrm{TiCr}$ spectral lines were weak, we can only infer an estimate of the $T_{\mathrm{e}}$ from the data. The most plausible explanation for the discrepancy between calculations and experiments is that the tracer dot remains well localized and moves out of the laser beam path, thereby sampling a cooler plasma.

\section{$T_{\mathrm{e}}$ Measurements}

We designed this set of experiments to measure the $T_{e}$ of gas-filled hohlraum targets. The target was a 2.5-mm-diam and 2.5-mm-long Au hohlraum. To confine the gas, all openings were covered with thin polyimide windows $(1 \mu \mathrm{m}$ thick over the diagnostic holes, $6000 \AA$ thick over the laser entrance holes). The hohlraum was filled with neopentane gas $\mathrm{C}_{5} \mathrm{H}_{12}$ and

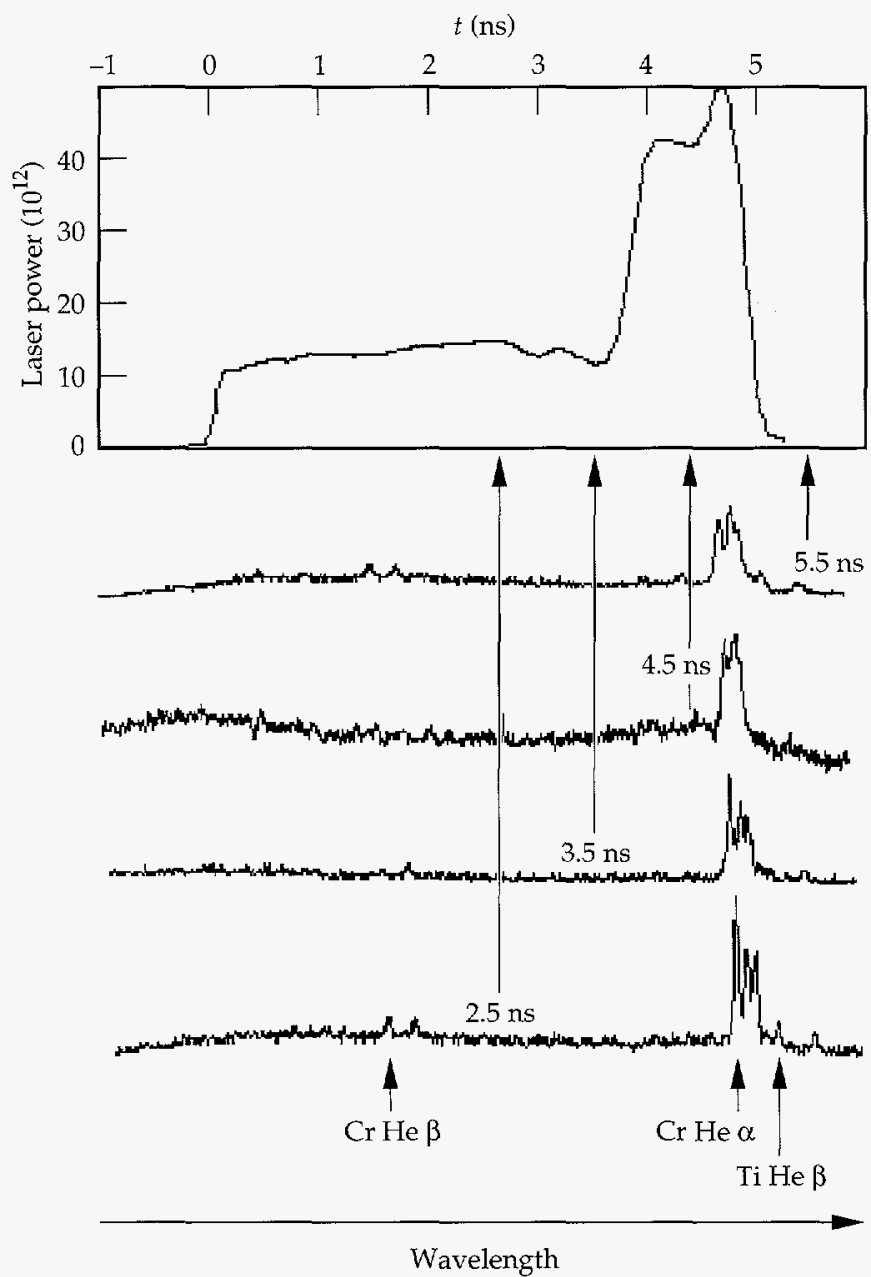

FIGURE 4. Data from a $\mathrm{CH}$ lined hohlraum showing a tracer embedded at $1 \mu \mathrm{m} . \quad(08-20-0695-1663 \mathrm{pb} 01)$ was designed to create a large millimeter-size plasma for stimulated Brillouin scattering and stimulated Raman scattering studies. ${ }^{8}$

These hohlraums are smaller than those used in the previous experiments and are expected to have a higher radiation temperature $T_{R}$. In the presence of an intense external photon flux, the ionic populations become more difficult to calculate because the introduction of the radiative transfer requires a solution of an integrodifferential equation which depends on the population and the field. Previous spectroscopic diagnostics cannot be extrapolated to these cases because they do not include the effects of an intense photon field. In the smaller hohlraums, the $T_{R}$ can be high enough to actively perturb the level populations of the dopants and thereby will change the standard dependencies of these level populations on the $T_{e}$ and $n_{e}$. This means that the ratios will now have an added dependence on the radiation field, which was previously assumed negligible. This radiation field will tend to deplete lower level populations by photoionization and photoexcitation and will cause mixing between excited levels, as they thermalize with the radiation field. Since these effects depend on the population and depopulation of energy levels, which are intricately coupled to the radiation field, and the plasma response to that photon field, the ratios will depend not only on the $T_{R}$, but on the detailed spectral structure of the radiation field.

To analyze the spectra from gas-filled hohlraums, the radiation field generated inside the 2.5 - $\mathrm{mm}$-diam hohlraums was measured by an x-ray diode diagnostic called Dante. ${ }^{9}$ These experiments provided data that were used as input to the spectroscopic plasma models. Figure 5 shows the spectra of the radiation drive obtained at the peak of the pulse. Overall, the peak $T_{R}$ was $\sim 190 \mathrm{eV}$. In the experiments, the diagnostic observed

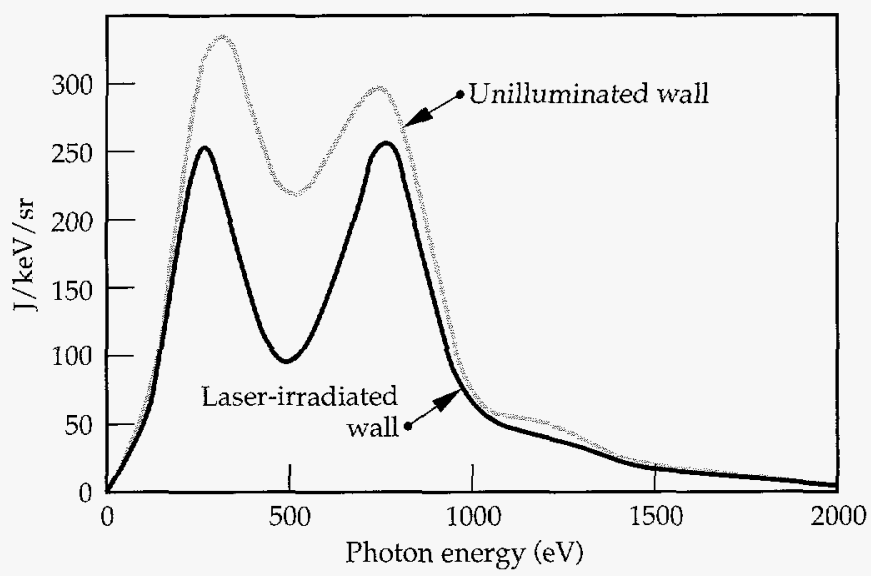

FIGURE 5. Dante spectra of the flux from a $2.5-\mathrm{mm}$-diam hohlraum at the peak intensities generated during the pulse. $M$ bands are not shown. (08-20-0695-1665pb01) 
either a beam focal spot or an area that was not irradiated by the beam. The measurements allow a comparison of the frequency dependence of the radiation

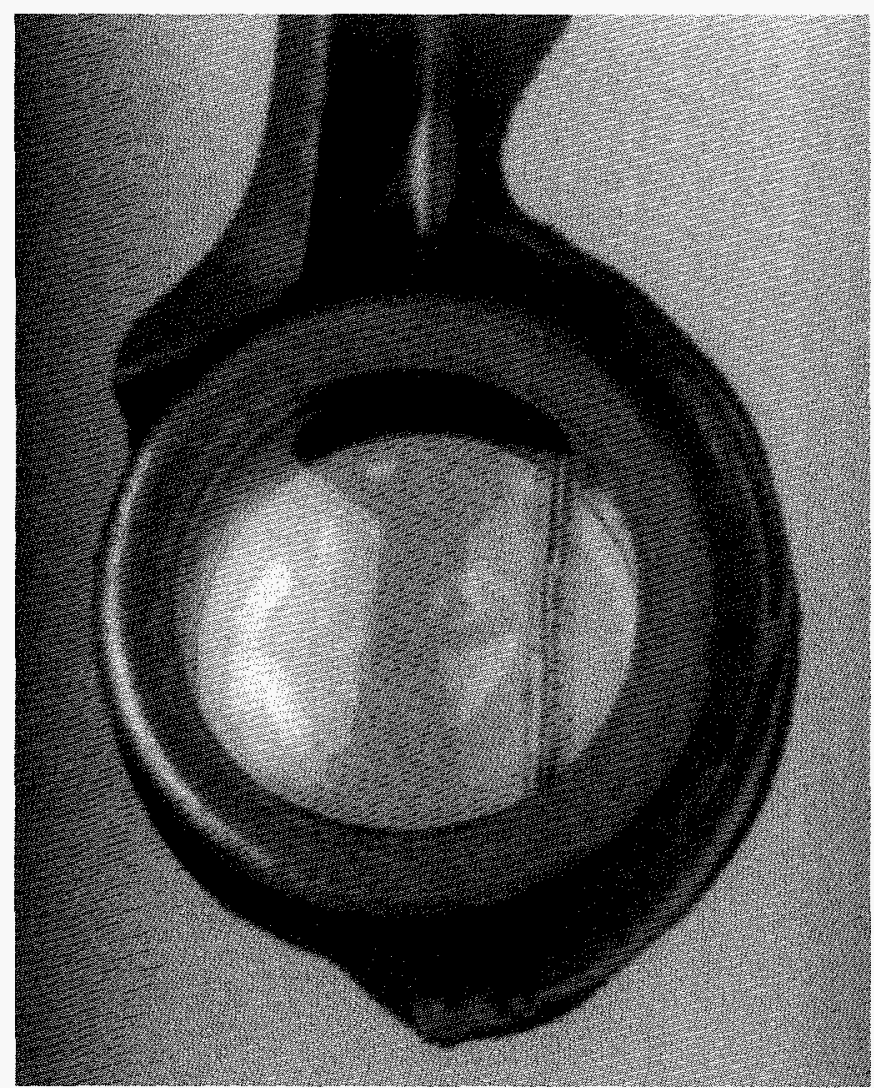

FIGURE 6. Photograph of a foil suspended in a hohlraum target. (10-00-0394-0788 Apb01)
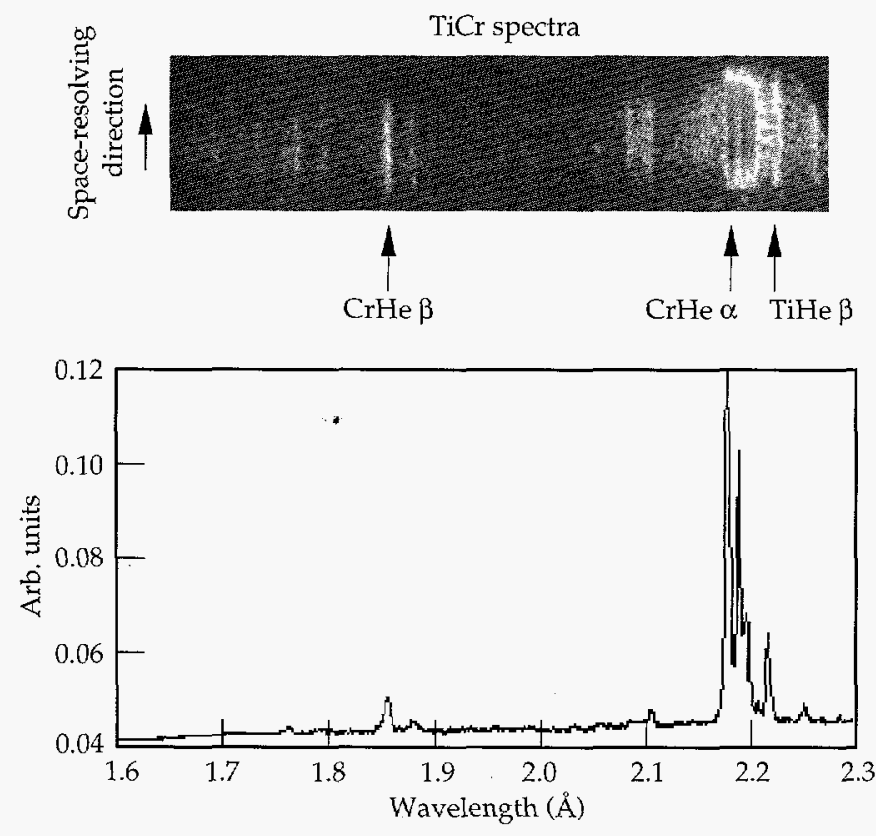

FIGURE 7. Data and lineout of the $x$-ray emission from a hohlraum target. (08-20-0695-1664pb01) field for these two cases. As shown in Fig. 5, both spectra are non-Planckian and there is a marked difference in the relative intensities of the $\mathrm{O}$ and $\mathrm{N} \mathrm{spec}-$ tral bands. In general, measurements observing a laser focal spot on the wall have more pronounced $N$ and $\mathrm{M}$ bands. For the $T_{\mathrm{R}}$ measured in these types of targets, the radiation effects on the spectra are not significant enough to affect the temperature measurements. This effect does become important in targets with higher $T_{\mathrm{R}}$ and research in this area continues.

To measure the $T_{e}$ in these hohlraums, a tracer plasma was formed from a $2000 \AA$ deposit of Ti and Cr cosputtered onto a $800 \AA$ thick $\mathrm{CH}$ foil substrate. Figure 6 is a photograph of the target, which shows a vertical foil suspended in the hohlraum. The measurement is based on a technique which uses isoelectronic lines from ionized plasmas to diagnose $T_{\mathrm{e}}{ }^{10}$ Briefly described, it is a line intensity ratio of emission from the same transition originating from two different ionic species having the same ionization stage. For instance, in this case we use the $\mathrm{Cr} \mathrm{He} \beta$ resonance line, $1 \mathrm{~s}^{2}\left({ }^{1} \mathrm{~S}_{0}\right)-1 \mathrm{~s} 3 \mathrm{p}\left({ }^{1} \mathrm{P}_{1}\right)$, and the Ti He $\beta$ resonance line.

Because of the potential nonuniformity of the inside of the hohlraum, the tracer is deposited in a $100-\mu \mathrm{m}$-wide strip that is suspended in the hohlraum with the deposit facing the beam, as shown in Fig 1. Figure 7 shows an example of the spectra that have been corrected for the instrument response and gain. The experimental data, represented by circles in Fig. 8 , show that $T_{e} \geq 3 \mathrm{keV}$ for $500 \mathrm{ps}$. The full results and other experimental details of the measurements will be reported elsewhere. ${ }^{11}$

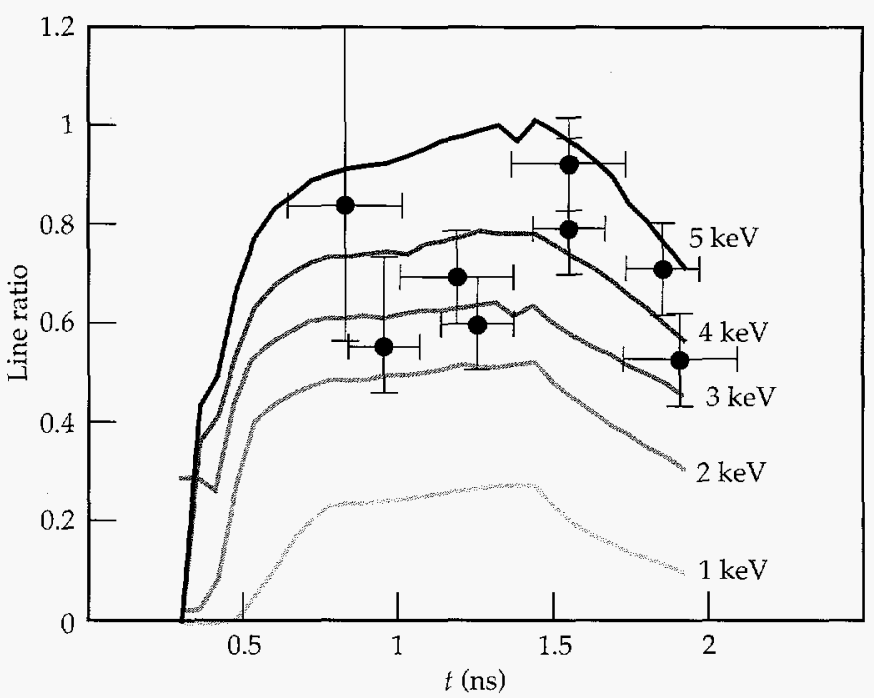

FIGURE 8. Example of data from a hohIraum target. (08-20-0895-1871pb01) 


\section{Summary}

To achieve fusion by indirect drive, a confined cavity creates an $\mathrm{x}$-ray source to provide proper symmetry for the implosion of a microballoon. The experiments described here are designed to diagnose the hohlraum plasma conditions by spectroscopy. The detailed behavior of hohlraums can be explored by these techniques because tracers can diagnose the local plasma conditions.

Experiments have demonstrated that line emission from microdot tracer plasmas can be observed above background emission. Emission from dopants on the hohlraum wall have revealed that spectra obtained from lined hohlraums have a temporal delay and are not as intense as those of unlined hohlraums. Recent results from lined and gas-filled hohlraums have shown that the $T_{e}$ can be diagnosed by a line intensity ratio technique. Large lined hohlraums achieve temperatures of $<2 \mathrm{keV}$ while gas-filled $2.5-\mathrm{mm}$ hohlraums reach $T_{e}>3 \mathrm{keV}$. Near-term experiments will develop the spectroscopic techniques to test the effect of high radiative fluxes on the plasma kinetics models and will explore the time-dependent effects.

\section{Notes and References}

1. W. C. Mead, et al., Phys. Fluids 26, 2316 (1993).

2. H. Pepin, R. Fabbro, B. Farai, F. Amiranoff, et al., Phys. Fluids 28, 3393 (1985); D. Phillion and C. J. Hailey, Phys. Rev. A 34, 4886 (1986).

3. C. L. S. Lewis and J. McGlinchey, Opt. Commun. 53, 179 (1985); A. Hauer, R. D. Cowan, B. Yaakobi, O. Barnouin, and R. Epstein, Phys. Rev. A 34, 411 (1986); B. A Hammel et al., Phys. Rev. Lett. 70, 1263 (1993).

4. B. Thomas, S. J. Davidson, C. C. Smith, and K. A. Warburton, ACO-UK-7839, 1985; R. Sigel, et al., Phys. Rev. Lett. 65, 587 (1990); R. Weber, P. F. Cunningham, and J. E., Appl. Phys. Lett. 53, 2596 (1988); R. L. Kauffman, et al., Phys. Rev. Lett. 73, 2320 (1994); H. Nishimura, H. Takabe, K. Kondo, T. Endo, et al., Phys. Rev. A 43, 3073 (1991).

5. P. G. Burkhalter, M. J. Herbst, D. Duston, J. Gardner, et al., Phys. Fluids 26, 3650 (1983); M. J. Herbst, P. G. Burkhalter, J. Grun, R. R. Whitlock, and M. Fink, Rev. Sci Instrum. 53, 1418 (1982); J. J. Herbst and J. Grun, Phys. Fluids 24, 1917 (1981).

6. T. Shepard, Lawrence Livermore National Laboratory, Livermore, CA, private communication (1994).

7. C. A. Back, R. L. Kauffman, P. M. Bell, J. D. Kilkenny, Rev. Sci. Instrum. 66, 764 (1995).

8. L. V. Powers, et al., Phys. Rev. Lett. 74, 2957 (1995).

9. H. N. Kornblum, R. L. Kauffman, and J. A. Smith, Rev. Sci. Instrum. 57, 2179 (1986).

10. T. D. Shepard, C. A. Back, B. H. Failor, W. W. Hsing, et al., ICF Quarterly Report 4(4), 137-144, Lawrence Livermore National Laboratory, Livermore, CA, UCRL-LR-105821-94-4 (1994).

11. C. A. Back, D. H. Kalantar, B. J. MacGowan, R. L. Kauffman, et al., "Measurements of Electron Temperature by Spectroscopy in Millimeter-Size Hohlraum Targets," Lawrence Livermore National Laboratory, Livermore, CA, UCRL-JC-122046 (in progress, 1995), in preparation for Phys. Rev. Lett. 


\title{
MODELING THE EFFECTS OF LASER-BEAM SMOOTHING ON FILAMENTATION AND STIMULATED BRILLOUIN BACKSCATTERING
}

\author{
R. L. Berger \\ C. W. Still \\ S. N. Dixit \\ T. B. Kaiser \\ A. B. Langdon \\ D. I. Eimerl \\ B. F. Lasinski \\ E. A. Williams \\ D. Pennington
}

Using the three-dimensional code (F3D), we compute the filamentation and backscattering of laser light. The results show that filamentation can be controlled and stimulated Brillouin backscattering (SBBS) can be reduced by using random phase plates (RPP) ${ }^{1}$ and small $f$-numbers or smoothing by spectral dispersion $(\mathrm{SSD})^{2}$ with large bandwidth. An interesting result is that, for uniform plasmas, the SBBS amplification takes place over several laser axial coherence lengths (coherence length $\approx$ speckle length $\approx 8 f^{2} \lambda_{0}$, where $\lambda_{0}$ is laser wavelength).

\section{Introduction}

Controlling stimulated Brillouin scattering (SBS) and filamentation are essential to the success of laser fusion because together they affect the amount and location of laser energy delivered to the hohlraum wall for indirect drive and to the absorption region for direct drive. Filamentation and self-focusing occur when a density depression is produced by the nonuniform light intensity through ponderomotive and thermal pressures. Light refracts toward lower densities, and the light intensity increases until diffractive losses limit the lateral dimension of the nonuniformity. The incident laser beam has strong intensity nonuniformities, so that even modest filamentation gain may be unacceptable. Moreover, filamentation is the suspected reason that significant levels of SBS and stimulated Raman scattering (SRS) are observed even when calculated gain exponents are modest $(G<5)$. Because the length of laser beam hotspots is much larger than the width (ratio of length to width is $\sim 8 f$ ), backscattering is expected to be more important than sidescattering. Without hotspots, the gain rate and the growth rate of backscattering in a uniform plasma are not much different from those of sidescattering. For example, if the acoustic wave is weakly damped, the backscattering
Brillouin growth rate is only $2^{1 / 4}$ times the sidescatter rate. Laser-beam smoothing schemes such as SSD and induced spatial incoherence (ISI) ${ }^{3}$ illuminate the target at best focus where the laser spot is comprised of a large number of diffraction-limited hotspots. Therefore, understanding the laser-plasma interaction with hotspots is essential. We illustrate the effects of filamentation in Figure 1 where surfaces that enclose volumes in which laser intensity is higher than five times the average intensity are shown. Figure 1(a) shows the surfaces before any self-focusing has developed.

Figure 1(b) shows the surfaces after the filaments have developed. There is an obvious increase in the number of high-intensity regions.

In previous reports ${ }^{4,5}$ we presented the equations, the approximations and their justification, the numerical techniques, and some results obtained with the F3D code. This code does three-dimensional (3-D) calculations of the propagation of laser beams in which the laser light self consistently filaments and Brillouin backscatters. (a) Before filamentation

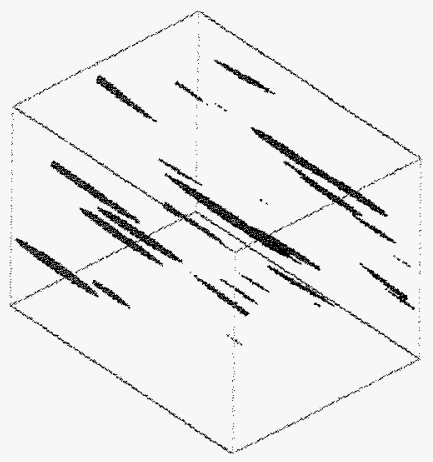

(b) After filamentation

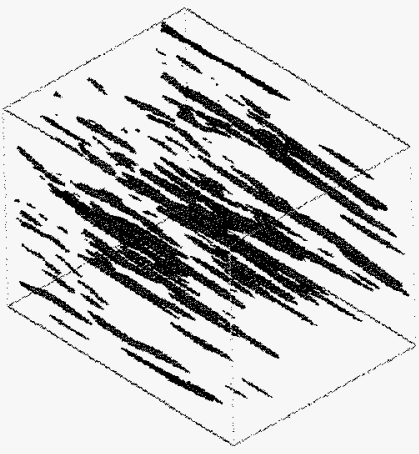

FIGURE 1. Three-dimensional surfaces within which the laser intensity is greater than five times average (a) before filamentation and (b) after filamentation. (50-01-0895-1872pb01) 
Here, we review laser-beam smoothing techniques and present the effects of temporal beam incoherence on filamentation and SBBS. Because filamentation and backscattering are the dominant interactions in the problems we consider, we can separate the light wave into a nearly forward- and nearly backward-moving wave, each treated within the paraxial approximation. Similarly, we can separate the acoustic wave response into long-wavelength modes, driven by filamentation or forward scattering, and short-wavelength modes, driven by backscattering. This separation allows us to consider fairly large regions of underdense plasma, containing many hotspots, because the spatial resolution necessary with this scheme is much less than in treatments that solve the full wave equation. ${ }^{6}$ This allows us to consider the influence of SSD and other temporal beam-smoothing techniques on filamentation and SBBS.

\section{Effect of Laser-Beam Smoothing on Filamentation}

In the focal spot of a laser beam focused with an RPP, the laser intensity is highly modulated with a $\operatorname{sinc}^{2}(X)$ $\operatorname{sinc}^{2}(Y)$ envelope; here $X=\pi x d_{x} / f_{1} \lambda_{0}, Y=\pi y d_{y} / f_{1} \lambda_{0}$, where $d_{x}$ and $d_{y}$ are the RPP element sizes in the transverse $x$ and $y$ directions, $f_{1}$ is the lens focal length, and $\lambda_{0}$ is the laser wavelength. On the smaller scale of the diffraction-limited spot for the full lens aperture $D$, there are hotspots (speckles) with a distribution of intensities up to $N$ times the average, where $N=\mathrm{D} / d_{x}$ for square RPP array. The laser beam can focus on the scale of the laser spot $f_{1} \lambda_{0} / d_{x}$ (whole-beam self-focusing), on the scale of the speckles $f \lambda_{0}=f_{1} \lambda_{0} / D$ (filamentation), and on scales between these limits (filamentation).

Laser-beam smoothing is primarily intended to suppress the filamentation process, which occurs on a much shorter time scale than whole-beam effects. Here, we consider the evolution of filamentation for a representative portion of the beam because simulation of the whole beam, including the small-scale structure, cannot be done for realistic laser beam diameters $\left(>1000 \lambda_{0}\right)$. In previous reports ${ }^{4,5,7}$ we showed that filamentation is stable if the length of the speckle $l_{s}$ is shorter than the minimum spatial gain length $l_{g}$, where

$1_{g}^{-1}=K_{\max }=0.125 \frac{v_{0}^{2}}{v_{e}^{2}} \frac{n_{\mathrm{e}}}{n_{\mathrm{c}}} \frac{\omega_{0}}{c}$

with $\omega_{0} / c=2 \pi / \lambda_{0} . K$ is the filamentation spatial gain rate, $c$ is the speed of light, $n_{\mathrm{e}}$ is the electron density, $n_{\mathrm{c}}$ is the critical density, $v_{0}=\mathrm{eE} / m_{\mathrm{e}} \omega_{0}$ is the jitter velocity of an electron in the laser electric field, and $v_{\mathrm{e}}$ is the electron thermal velocity. An equivalent statement is that stability against filamentation requires that the gain exponent for filamentation in a plasma one speckle length long be less than unity.

When this criterion is not satisfied, temporal smoothing is required to stabilize filamentation. For example, we have $l_{g}<l_{s}$ for parameters appropriate to the National Ignition Facility (NIF) design ${ }^{8,9}$ (e.g., for $f / 8,0.1 n_{\mathrm{c}^{\prime}}$ electron temperature $T_{\mathrm{e}}=3 \mathrm{keV}$, intensity $I=2 \times 10^{15} \mathrm{~W} / \mathrm{cm}^{2}$, $\lambda_{0}=0.351 \mu \mathrm{m}$, we have $l_{g}=0.8 l_{s}$ and $l_{s}=180 \mu \mathrm{m}$ ). For an ISI or SSD scheme, the speckles dissolve and reform in different locations on the time scale of the laser coherence time $\Delta \omega^{-1}$. Conversely, the intensity in a speckle increases at the rate $K c$; thus, we estimate that SSD or ISI will stabilize filamentation if $\Delta \omega>K c$. The criterion $\Delta \omega>K_{c}$ corresponds to

$\frac{\Delta \omega}{\omega_{0}}=\frac{\Delta \lambda}{\lambda_{0}}>4.6 \times 10^{-4} \frac{n_{\mathrm{e}}}{10^{21} \mathrm{~cm}^{-3}} \frac{I}{2 \times 10^{15} \mathrm{~W} / \mathrm{cm}^{2}}$

$\times \frac{3 \mathrm{keV}}{T_{\mathrm{e}}}\left(\frac{\lambda_{0}}{0.351 \mu \mathrm{m}}\right)^{4}$

Figures 2 and 3 show two measures of the effect of SSD bandwidth on filamentation. The simulation dimensions were typically 160 wavelengths along $x$ and $y$ and 530 wavelengths along $z$, the direction of propagation. Figure 2(a) shows the fraction, $F$, of laser beam energy above five times the average intensity as a function of laser bandwidth for a simulation case that was strongly unstable without SSD (i.e., for the NIF parameters listed previously but with laser intensity $4 \times 10^{15} \mathrm{~W} / \mathrm{cm}^{2}$ ). This fraction $F$ varies with $z$ from the initial value of $\sim 4 \%$ at $z=0$ to a maximum value followed in general by a decrease at larger $z$ as the beam breaks up. The fraction displayed is the maximum value. For sufficient bandwidth, this fraction is reduced to that for an RPP beam in vacuum. Figure 2(a) also shows the extent to which four-color illumination (described below) combined with SSD inhibits filamentation.

Figure 2(b) compares the distribution of intensities in several cases with that for an RPP beam in vacuum. ${ }^{10}$ The distributions plotted are for the simulation region in which the fraction shown in Fig. 2(a) is largest. In the no-SSD case (3120) beam energy is transferred to very high intensity at the expense of energy between 1-5 times the average. Note that this intensity-weighted distribution peaks at 2-3 $I_{0}$ in all cases, but the total energy is constant except for the loss to collisional absorption; thus the initial distribution has the most energy. The addition of a small bandwidth, $\Delta \omega / \omega_{0}=0.025 \%$ (case 3123), is not very effective, but it does reduce the population of the most intense hotspots. In the large-bandwidth case (3205), $\Delta \omega / \omega_{0}=0.15 \%$, very little energy is transferred to intensities $I>10 I_{0}$. In fact between 4 and $10 I_{0}$, this distribution has less energy than the initial RPP case. The fraction of beam energy at high intensity peaks near the region where the first foci occur and 
relaxes to a less energetic distribution at greater distances. However, this relaxation comes at the expense of increased beam divergence as the speckles get narrower and shorter.

Figure 3 shows contour plots of the total laser energy in transverse Fourier modes at a given $z$ vs $k_{x}$ and $k_{y}$ for the small and large SSD bandwidths. The incident wave has no energy for values of $\left|k_{x}\right|>k_{0} / 2 f=0.0625 k_{0}$ or $\left|k_{y}\right|>k_{0} / 2 f$ for an $f / 8$ lens. Filamentation breaks the beam into smaller-scale hotspots, which appear in this type of plot as an increase in the energy at higher $k_{\perp}$. Thus in Fig. 3(a), the filamented distribution shows a significant amount of energy outside the incident beam cutoff, whereas the SSD-stabilized case in Fig. 3(b) shows a small amount of energy at these $k_{\perp}$. These plots also represent the amount of energy outside a given angle in the near field (lens image plane) vs the angle. Such measurements are being made in Nova experiments. ${ }^{11}$
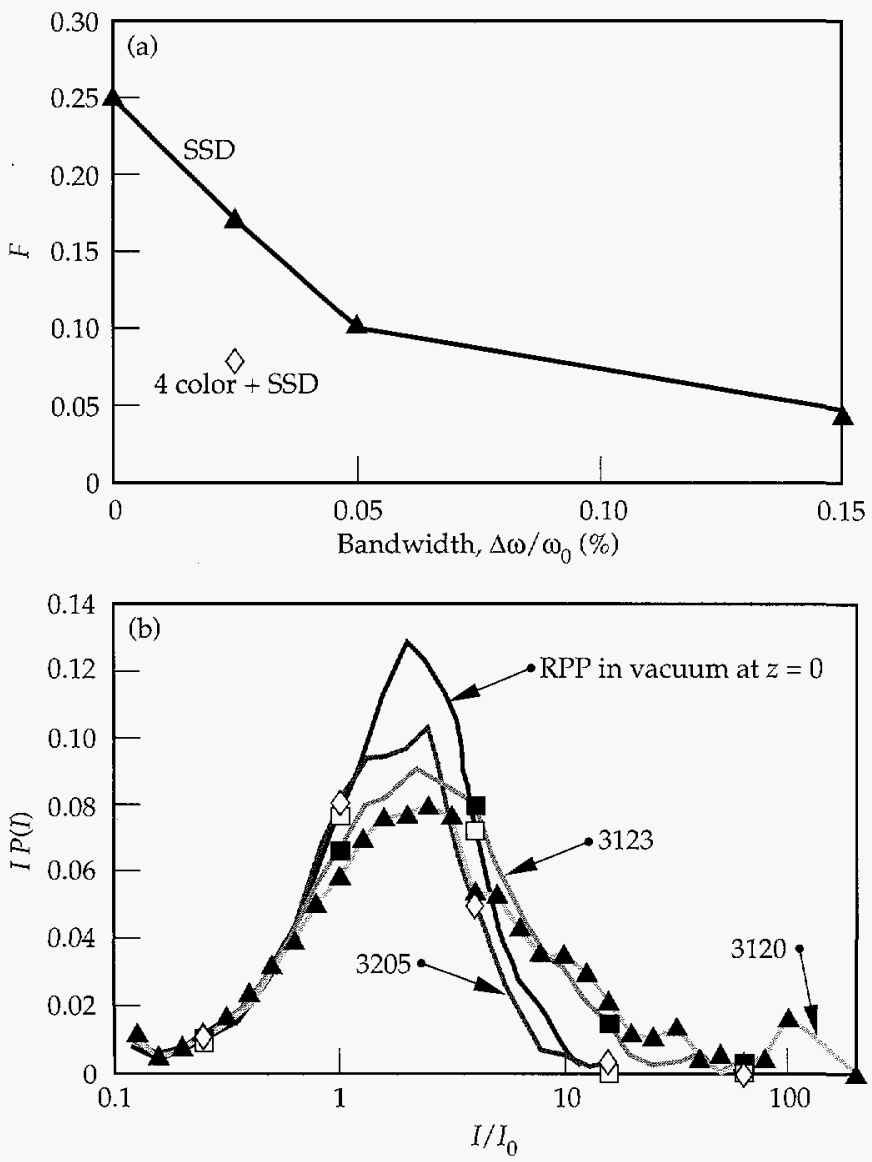

FIGURE 2. (a) Fraction $F$ of the laser beam energy above five times the average intensity as a function of the SSD bandwidth for an $f / 8$, $I_{0}=4 \times 10^{15} \mathrm{~W} / \mathrm{cm}^{2}$ laser beam. Also shown is one case combining four-color illumination with SSD. (b) The intensity weighted distribution. For the initial distribution at $z=0$, the integral of the distribution is unity (i.e., the average is $I_{0}$ ), but it is less than unity for $z>0$ because of collisional absorption. (50-01-0895-1882pb01)
For $3 \omega$ illumination, the bandwidth required to stabilize filamentation at intensities in excess of $2 \times 10^{15} \mathrm{~W} / \mathrm{cm}^{2}$ cannot be used because the tripling efficiency is too low for bandwidths $\Delta \lambda / \lambda_{0} \geq 3 \times 10^{-4}$. A different temporal scheme was proposed ${ }^{12}$ wherein four narrow-band laser beams with slightly different wavelengths are focused using different quadrants of the lens to overlap in the target plane. The interference pattern (the speckles) then moves periodically in time,

(a) Small SSD bandwidth case $3123(0.025 \%)$

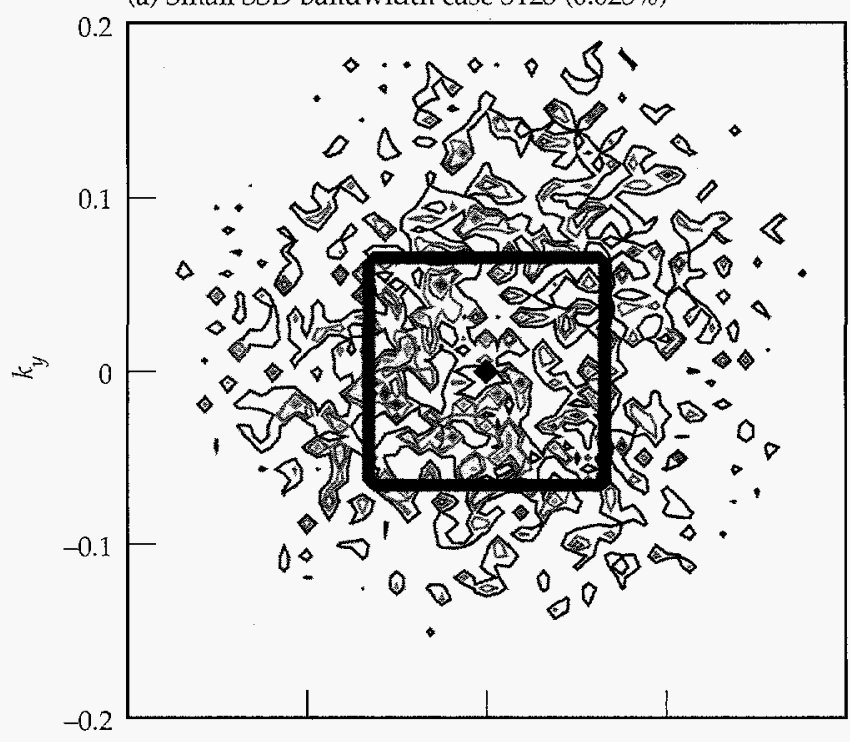

(b) Large SSD bandwidth case $3205(0.15 \%)$

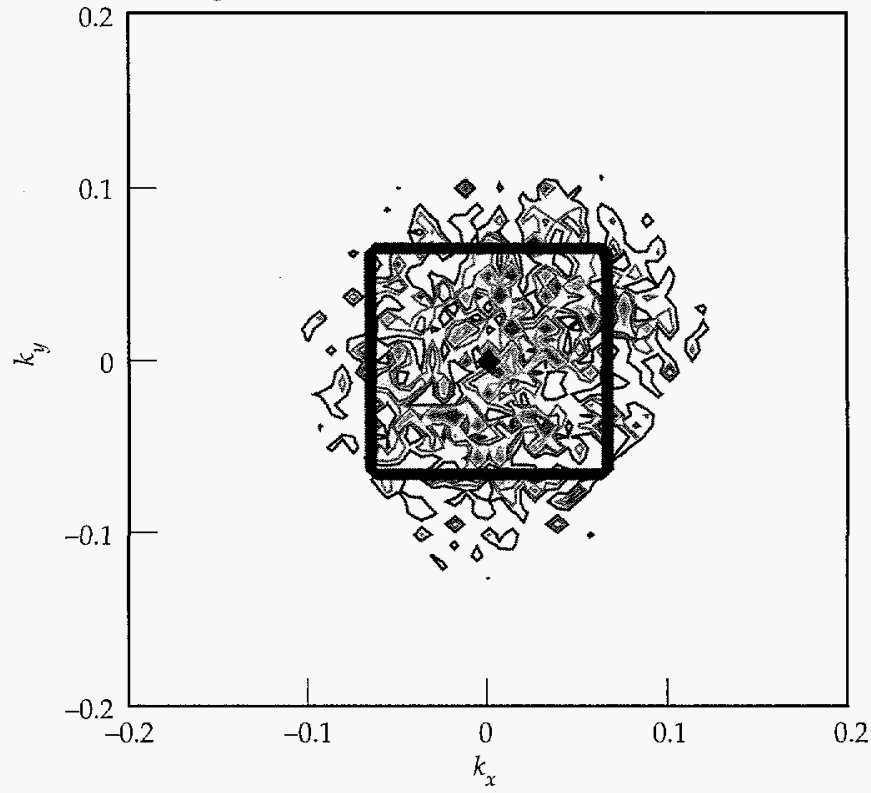

FIGURE 3. Fourier components of the laser beam energy with average $I_{0}=4 \times 10^{15} \mathrm{~W} / \mathrm{cm}^{2}$ for the incident beam at $z=0$, and after propagating $500 \lambda_{0}$ for (a) an SSD bandwidth of $0.025 \%$ and (b) an SSD bandwidth of $0.15 \%$. The incident laser beam energy is uniformly distributed over perpendicular wavelengths that fit within an $f / 8$ square aperture lens. $\quad$ (50-01-0895-1874pb01) 
not randomly as in an SSD or ISI scheme. If the frequency separation between adjacent lines is $\delta \omega$, the time $t_{\mathrm{r}}$ for the speckle pattern to repeat is $t_{\mathrm{r}}=2 \pi / \delta \omega$. The time-averaged speckle pattern is not smoothed because the $f / 16$ speckles produced by each quadrant of the lens are unaffected. This four-color scheme ${ }^{13}$ stabilizes filaments from the $f / 8$ structure but unfortunately does not stop filaments from forming due to the remaining static $f / 16$ speckles. Nonetheless, with fourcolor illumination, it is the intensity per quadrant that counts, and the spatial growth rate is decreased by a factor of four. However, since the speckle length has effectively increased by a factor of four, the net effect in plasmas longer than an $f / 16$ speckle is to increase the distance over which the beam propagates before breaking up. Filamentation stability can be recovered with the introduction of SSD with a bandwidth of $0.025 \%$ (if used in conjunction with four-color illumination) because the $f / 16$ speckles (and even longer wavelength structures) are now temporally smoothed. Equation (2) can be used to estimate the minimum four-color separation needed with $\delta \omega=\Delta \omega_{\min } / 3$ provided $\Delta \omega_{\mathrm{SSD}}>\Delta \omega_{\min } / 4$ For the nominal parameters of the NIF, $\Delta \lambda_{\min } \approx 0.5 \mathrm{~nm}$ before frequency tripling $\left(\Delta \lambda_{\min }=0.17 \mathrm{~nm}\right.$ at $\left.0.351 \mu \mathrm{m}\right)$, so an SSD bandwidth $\Delta \lambda_{\mathrm{SSD}}=0.25 \mathrm{~nm}$ is twice what is estimated as necessary.

This work only addresses the stability of the speckles on the small scale. Focusing can also occur on the larger scale of the whole beam. Four-color illumination and SSD will have little effect on that process. Dixit has pointed out ${ }^{14}$ that the grating dispersion need only be large enough to displace the hotspots by a speckle width to temporally smooth the intensity pattern, at least in a model that neglects phase errors and lens aberrations. We surmise that, in the more general case, laser beams may filament on scales intermediate between the speckle size and the whole beam, and larger grating dispersion may help in this case.

\section{Effect of Laser-Beam Smoothing on Stimulated Brillouin Backscattering}

The spatial structure of the laser beam on the scale of a speckle and temporal smoothing of the hotspots have an effect on the spatial and temporal growth of SBBS. For SBS, there is an additional effect possible that survives in a 1-D treatment even if the hotspots are stationary, namely that the convective or early-time growth rate of SBS is reduced if $\gamma_{0}<\Delta \omega$ and $\Delta \omega>\operatorname{Max}$ $\left(v_{a}, v\right)$, where $v_{a}$ and $v$ are the damping rates of the acoustic and light wave, respectively. The weakly coupled SBBS growth rate $\gamma_{0}$, given by

$$
\begin{aligned}
& \frac{\gamma_{0}}{\omega_{0}}=\frac{1}{4} \frac{v_{0}}{v_{e}} \frac{\omega_{p e}}{\omega_{0}}\left(\frac{\omega_{a}}{\omega_{0}}\right)^{1 / 2} \\
& \cong 8 \times 10^{-4}\left(\frac{I}{2 \times 10^{15} \mathrm{~W} / \mathrm{cm}^{2}}\right)^{1 / 2}\left(\frac{\lambda_{0}}{0.351 \mu \mathrm{m}}\right)^{2} \\
& \left(\frac{n_{\mathrm{e}}}{10^{21} \mathrm{~cm}^{-3}}\right)^{1 / 2} \times\left(\frac{3 \mathrm{keV}}{\mathrm{T}_{e}}\right)^{1 / 4}\left(\frac{Z}{A}\right)^{1 / 4},
\end{aligned}
$$

is in general larger than the laser bandwidth, especially in the hotspots. For multispecies plasmas, $Z / A \rightarrow\left(Z_{j}^{2} / A_{j}\right) /\left(Z_{j}\right)$, where the averages of the charge states $Z$ and atomic numbers $A$ are taken over the ion species $j$. This expression for $\gamma_{0}$ applies if $Z T_{\mathrm{e}} / T_{\mathrm{i}} \geq 3$ for all species in multispecies plasma and $k \lambda_{D e}<1$ where $\lambda_{D e}$ is the electron Debye length. If the fluid approximation for either species does not apply, the frequency and damping characteristics of the acoustic mode are significantly modified. ${ }^{15}$ For narrowband four-color illumination, $\Delta \omega$ can be larger than $\gamma_{0}$, and a reduction in SBS without beam smoothing might occur. However, in our simulations, since four-color illumination always causes the hotspots to move, the pure bandwidth effect on SBS has not been studied. In summary, we expect no effect on SBS from bandwidth without smoothing (i.e., there is no dispersion and the speckles are stationary); none is observed in our $\gamma_{0}<\Delta \omega$ simulations.

As discussed in the previous section, the laser speckle size in the focal plane region affects the stability of the laser light against filamentation. Our initial expectation was that the SBBS would occur independently in each speckle and thus be very sensitive to the laser $f$-number, ${ }^{16}$ but our simulations in uniform plasmas showed that the spatial amplification occurred over many speckle lengths. The primary determinant of the reflectivity, when the laser intensity is below the absolute growth threshold, is the convective gain exponent

$G=\frac{1}{8} \frac{v_{0}^{2}}{v_{e}^{2}} \frac{n_{\mathrm{e}}}{n_{\mathrm{c}}} \frac{\omega_{a}}{v_{a}} \frac{\omega_{0} L}{c}$,

where $L$ is the axial system length. Note that the spatial gain rate for SBBS is higher than that for filamentation by the ratio $\omega_{a /} v_{a}$. This fact has consequences in the simulations, which we discuss later.

Figure 4 shows the SBBS reflectivities from our simulations without temporal smoothing as a function of $G$ for $f / 8$ and $f / 4$ laser illumination. The $f / 8$ reflectivity is systematically higher than that for $f / 4$ for the same plasma conditions and laser intensity. The difference is much less than a single-hotspot model would predict because, then, the gain exponent per speckle would be 
the figure of merit. For example, for a single-hotspot model, the $f / 4$ reflectivity with a gain exponent of 20 should be four times the $f / 8$ value with a gain exponent of 5 if all other factors were constant. The factor of four comes from the fact that the number of hotspots per axial length is larger for $f / 4$. That clearly is not the result of the simulations.

Another measure of $f$-number effects is the gain exponent above which significant reflectivity occurs. Figure 4 indicates that this gain exponent is $\sim 4$ for $f / 8$ and $\sim 6$ for $f / 4$; these exponents are in the ratio 1.5 , not $(8 / 4)^{2}=4$, as would be expected from a singlehotspot model.

Some of the difference between the $f / 8$ and $f / 4$ simulations may be the result of some filamentation in the $f / 8$ case since, for intensities higher than $\sim 2 \times 10^{15} \mathrm{~W} / \mathrm{cm}^{2}$, the $f / 8$ laser light is unstable against filamentation. Simulations for $f / 8$ in which the light refraction was neglected (but the hotspots remained) showed about a factor of two decrease in reflectivity at $G=12$. Another reason may be incomplete phase conjugation, as discussed below.

We have concentrated on modeling laser and plasma processes relevant to the NIF and to current Nova experiments. For these parameters, filamentation in fact has less influence on the SBBS results than we expected. First, with average laser intensities less than $5 \times 10^{15} \mathrm{~W} / \mathrm{cm}^{2}, \mathrm{f} / 4$ speckles are stable against filamentation. With $f / 8$ focusing, filamentation is important above $2 \times 10^{15} \mathrm{~W} / \mathrm{cm}^{2}$, but then the SBS gain is so high in our uniform plasma simulations (for the range of damping rates used) that the laser intensity becomes depleted before filaments fully develop. That is, as the hotspots start to focus, the laser intensity increases,

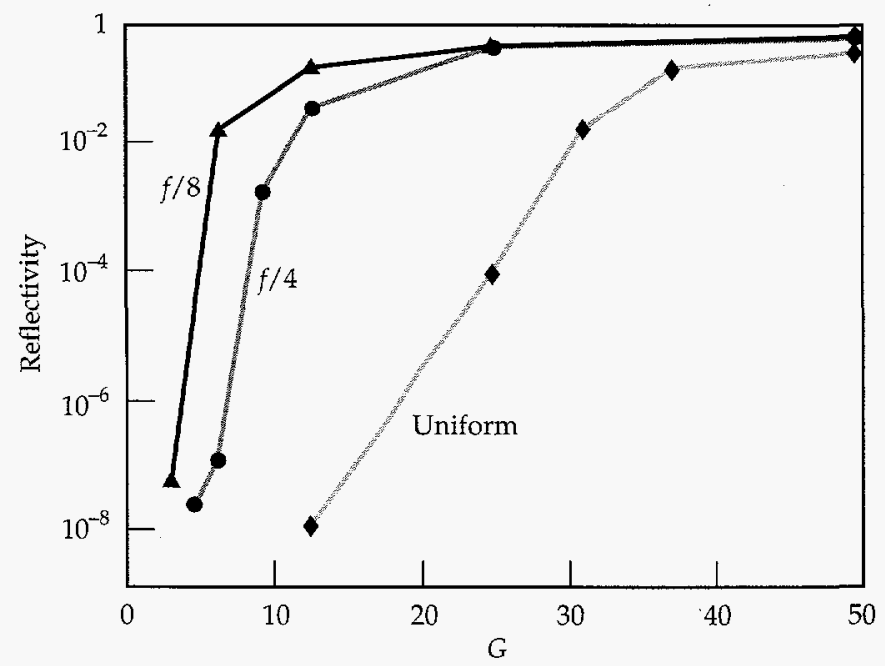

FIGURE 4. SBS reflectivity vs 1-D gain exponent for temporally unsmoothed $f / 8, f / 4$, and uniform laser beams. $\quad(50-01-0895-1876 \mathrm{pb} 01)$ which increases the growth rate of SBBS. Then the backward-moving light robs power from the forwardmoving light, making the rate of focusing less than it would be without SBBS. If the gains were lower, filamentation would cause more of a difference between $f / 4$ and $f / 8$ reflectivities. In reality, SBBS may saturate before the laser intensity is depleted because of nonlinear limits on the SBBS growth; ${ }^{17}$ this is the focus of our current research. ${ }^{18}$

A heuristic explanation for the weak dependence of SBBS on the $f$-number involves the notion of phase conjugation. ${ }^{19}$ The pattern of speckles at any $z>0$ for the incident laser light is determined by the amplitude and phase of the transverse Fourier components at $z=0$. A light wave of nearly the same frequency propagating in the backward direction, e.g., an SBBS wave, would have the same pattern of speckles between zero and $z$ if its components had the same relative amplitude but the conjugate phase of the incident light at $z$. (Of course, this is only true if filamentation, sidescattering, or other nonlinear processes do not alter the propagation substantially.) From all the light waves that the plasma produces as a result of collisional emission or Thomson scattering, those whose phase and Fourier components match those of the incident light will be amplified most because, over many speckle lengths, their hotspot patterns overlap that of the incident light. As yet, we do not have a statistical measure of the degree of phase conjugation; however, we have examined sequences of 2-D $x-y$ plots comparing the incident-beam and reflectedlight hotspots at several planes in $z$ separated by more than a speckle length. The reflected-light hotspots are always associated with a laser-beam hotspot.

Now, consider a plasma one $f / 8$ speckle length long. For $f / 8$ illumination, the SBBS will grow in hotspots of about the same length as the plasma and will experience a gain in excess of the uniform-intensity gain. The $f / 4$ SBBS will initially grow in the backward direction in a hotspot of 0.25 the plasma length, and, if phase conjugation does not occur, it will grow at a reduced rate once the waves leave their hotspots. However, because of the collective effect of phase conjugation, the backward light wave's hotspots overlap those of the incident laser wave and continue to drive ion acoustic waves efficiently through the ponderomotive force (proportional to the product of the light wave amplitudes). Thus the SBBS grows in $f / 4$ hotspots almost as effectively as in the $f / 8$ hotspots.

As discussed earlier, the laser bandwidth available at $0.35 \mu \mathrm{m}$ on Nova is too low to reduce the amplification or growth rate directly. However, given that the SBBS grows in hotspots and takes many growth times to reach saturation, SSD or an equivalent beam-smoothing technique may be effective in reducing the reflectivity because the hotspots are no longer stationary. In addi- 
tion, if the gain occurs over an extended region of plasma, the phase coherence of the incident wave with the reflected wave will be reduced with the reduction in reflectivity.

Figure 5 shows the reflectivity as a function of $G$ for $f / 4$ illumination with and without temporal beam smoothing. The results for one-color, fourcolor, SSD (nominal and large-bandwidth), and a combination of four-color with SSD are shown. The adjacent color separation $\delta \lambda$ and the laser bandwidth $\Delta \lambda$ were chosen as appropriate for Nova experiments, namely $\delta \lambda=0.42 \mathrm{~nm}$ and $\Delta \lambda=0.25 \mathrm{~nm}$ at $1.06 \mu \mathrm{m}$. The ratios $\delta \omega / \omega_{0}$ and $\Delta \omega / \omega_{0}$ of frequency separation and bandwidth to laser frequency are assumed to be preserved by frequency tripling or doubling. All the reflectivities shown in Fig. 5 exceed those produced by bremsstrahlung emission or by Thomson scattering from thermal ion acoustic fluctuations. The one-color results are the same as shown in Fig. 4. Below $G=10$, the effect of beam smoothing is quite dramatic; the reflectivities drop below $10^{-6}$ for $G \leq 6$.

Other $f / 4$ simulations have been done with different damping rates, plasma lengths, and laser intensities. At $G \approx 12$, the SSD reflectivities for different simulations vary by five orders of magnitude. The highest reflectivity (1.8\%) occurs at the highest intensity, $4 \times 10^{15} \mathrm{~W} / \mathrm{cm}^{2}$, with $L=515 \lambda_{0}$ and $v_{a} / \omega_{a}=0.2$; the lowest reflectivity $\left(2 \times 10^{-7}\right)$ occurs at the lowest intensity, $1 \times 10^{15} \mathrm{~W} / \mathrm{cm}^{2}$, with $L=515 \lambda_{0}$ and $v_{a} / \omega_{a}=0.05$. Increasing the intensity by a factor of two and halving the length to keep $G$ constant also results in higher reflectivity. The reflectivity is increased by an order of magnitude, from $2 \times 10^{-6}$ to $4 \times 10^{-5}$, by doubling the damping $v_{a} / \omega_{a}$ from 0.05 to 0.1 and $L$ from $256 \lambda_{0}$ to $515 \lambda_{0}$; it is increased by another order of magnitude to $2 \times 10^{-4}$ as $v_{a} / \omega_{a}$ increases to 0.2 and $L$ to $1030 \lambda_{0}$. Figure 6 shows these results. Both these trends would make sense if the addition of SSD bandwidth increased the effective acoustic wave damping to a value as high as $0.2 \omega_{a}$, so that the effective gain exponent increased with $L$ and/or $I$. This appears plausible because $\Delta \omega_{\mathrm{SSD}} / \omega_{0}=2.5 \times 10^{-4}$, whereas $v_{a} / \omega_{0} \cong 2\left(v_{a} / \omega_{a}\right) \times 10^{-3}=10^{-4}$ at the lowest damping rate. The light absorption rate $v=1 / 2\left(n_{\mathrm{e}} / n_{\mathrm{c}}\right) v_{e i}$ is even smaller: $v / \omega_{0} \approx 10^{-5}$ for $n_{\mathrm{e}}=10^{21} \mathrm{~cm}^{-3}, T_{\mathrm{e}}=3 \mathrm{keV}$, $\lambda_{0}=0.351 \mu \mathrm{m}$, and $Z_{\text {eff }}=5$ (where $v_{e i}$ is the electronion collision frequency).

In Fig. 5, the combination of four-color illumination and $0.025 \%$ bandwidth SSD brings the reflectivity below that for any four-color or $0.025 \%$ bandwidth SSD simulation at a given gain exponent, as one might expect. Only with gain exponents $G>20$ is there significant reflectivity; here, the variation of reflectivity with $G$ approaches that calculated for a uniform laser beam. The uniform laser beam reflectivities were calculated with $v_{a} / \omega_{a}=0.05$ and $L=515 \lambda_{0}$ and for various intensities up to $4 \times 10^{15} \mathrm{~W} / \mathrm{cm}^{2}$. At the highest gain exponents simulated, $G \approx 50$, all simulations with and without beam smoothing have high reflectivity, $R_{\mathrm{SBS}}>20 \%$, for which nonlinear saturation effects other than pump depletion are important. That is, these reflectivities are associated with large-amplitude acoustic waves $\left(\left|\delta n_{\mathrm{b}} / n\right|>0.5\right)$. A mere reduction in the local magnitude of $\delta n_{\mathrm{b}}$ without a corresponding limit on the length of plasma over which the waves remain in phase may not produce much reduction in reflectivity. That is, the laser will take longer to deplete but the overall reflectivity will stay nearly constant for large systems.

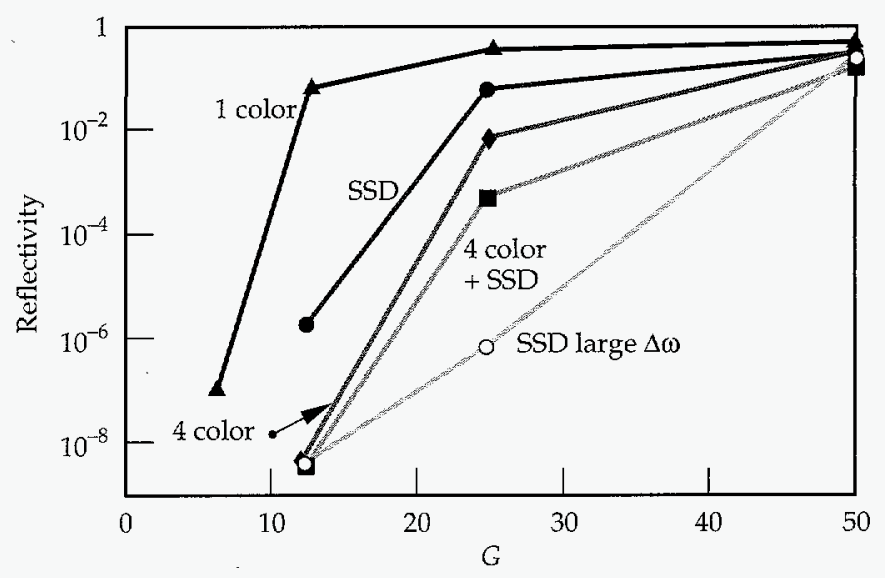

FIGURE 5. f/4 SBS reflectivity vs 1-D gain exponent for one-color, SSD, four-color, four-color plus SSD, and SSD with large bandwidth. (50-01-0895-1877pb01)

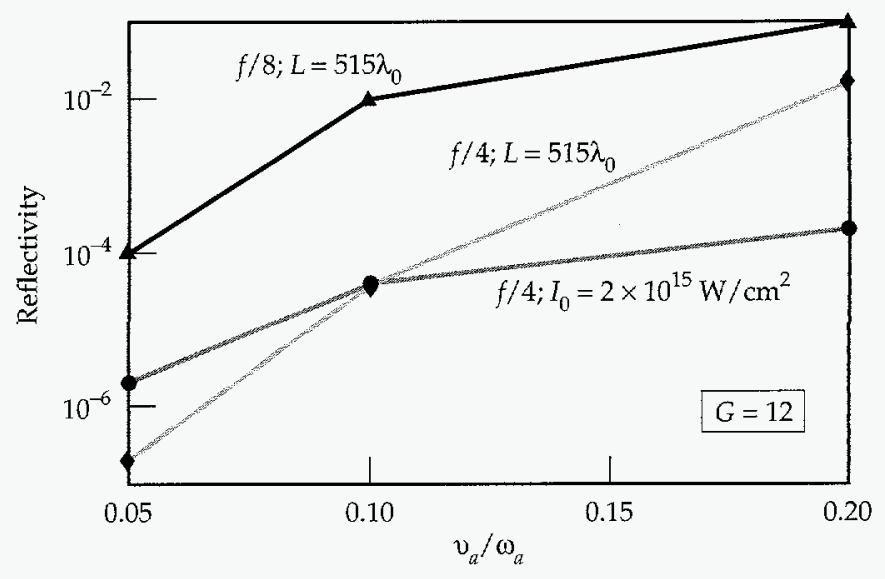

FIGURE 6. SBS reflectivity vs acoustic wave damping rate for a fixed gain exponent $G=12$ and either a fixed intensity and varying length for $f / 4$, a fixed length and varying intensity for $f / 4$, or a fixed length and varying intensity for $f / 8$. (50-01-0895-1878pb01) 
Figure 7 shows the $f / 8$ reflectivity calculations for one color, four colors, and four-colors plus SSD.

Fewer calculations were carried out for $f / 8$ than for $\mathrm{f} / 4$, but the benefit of the four-color plus SSD combination is also dramatic at $f / 8$. Since the speckle length is four times larger than for $f / 4$, the smallest length system is $515 \lambda_{0}$, one speckle length. The four-color scheme is not as effective at $f / 8$ as at $f / 4$ for moderate gain exponents $(G<20)$. The four-color plus SSD reflectivity shows the same trends with intensity and length as the $f / 4$ runs for SSD (see Fig. 6 ).

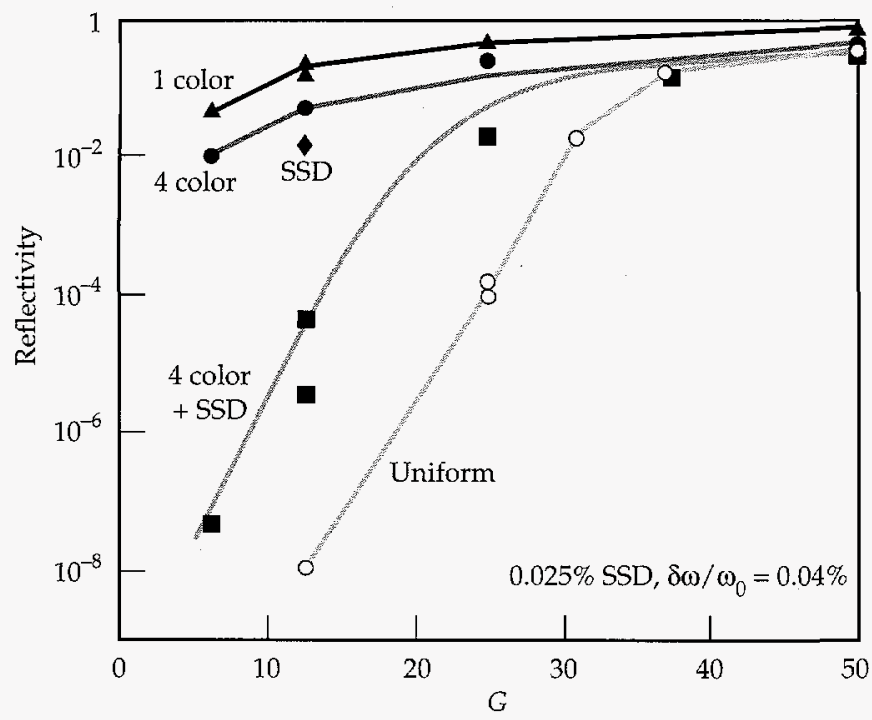

FIGURE 7. $f / 8$ SBS reflectivity vs 1-D gain exponent for one-color, four-color, SSD, four-color plus SSD, and uniform laser beams. (50-01-0895-1879pb01)

\section{Conclusions}

We have presented three-dimensional calculations of the propagation of laser beams in which the laser light self consistently filaments and Brillouin backscatters. We established that filamentation can be controlled by temporal and spatial beam smoothing for laser and plasma parameters of interest to the proposed NIF. Beam smoothing also reduces the SBBS reflectivity, especially when four-color illumination is combined with SSD. For the gain exponents expected in the NIF, and in experiments designed to reproduce the NIF conditions on Nova, ${ }^{8}$ the calculated reflectivities are about $10-20 \%$, whereas the observed reflectivities ${ }^{20,21}$ are less than $1-5 \%$. We believe that nonlinear processes, which are not yet modeled in this code, may explain the discrepancy, although other effects that limit the linear gain exponent ${ }^{22}$ may also be responsible. A simple nonlinear model ${ }^{23}$ (which had some success in modeling disk experiments in which velocity gradients also played a role) did not have much effect on the reflectivity unless the ion wave amplitudes were limited to lower values than typically observed in 1-D particle-incell simulations. Recent 2-D simulations have shown much lower ion wave amplitudes than 1-D simulations; we hope to incorporate this amplitude reduction into our code once the effects are understood well enough to reduce to a fluid model.

We also simulated SBBS in plasmas with strong velocity gradients, which limit the reflectivity to small values $\left(\sim 10^{-4}\right)$. We observed that the reflectivity was produced in one or two spots in which the local reflected light intensity was a significant fraction $\left(\sim 10^{-2}\right)$ of the local laser intensity. These simulations used the parameters of exploding-foil targets, ${ }^{24}$ for which images of the SBBS light were taken at the target plane. These images also showed that only a few spots were responsible for most of the light emission. The reflectivities in the simulations and the experiments were of similar magnitude.

\section{Acknowledgments}

We are pleased to acknowledge the benefit of discussions with the Lawrence Livermore National Laboratory plasma experiments group and D. Dubois, J. Kilkenny, W. L. Kruer, J. D. Lindl, L. V. Powers, H. Rose, M. D. Rosen, and S. C. Wilks. 


\section{Notes and References}

1. Y. Kato, et al., Phys. Rev. Lett. 53, 1057 (1984).

2. S. Skupsky, et al., J. Appl. Phys. 66, 3456 (1989).

3. R. H. Lehmberg and S. P. Obenschain, Opt. Commun. 46, 27 (1983).

4. R. L. Berger, et al., Phys. Fluids B 5, 2243 (1993).

5. R. L. Berger, et al., Phys. Rev. Lett. 75, 1078 (1995).

6. M. R. Amin, et al., Phys. Rev. Lett. 71, 81 (1993); ibid, Phys. Fluids $B$ 5, 3748 (1993).

7. C. Labaune, et al., Phys. Fluids B 4, 2224 (1992).

8. S. W. Haan, S. M. Pollaine, J. D. Lindl, L. J. Suter, et al., Phys. Plasmas 2, 2473 (1995).

9. L. V. Powers, R. L. Berger, D. H. Munro, and B. F. Lasinski, Phys. Plasmas 2, 2480 (1995).

10. H. A. Rose and D. F. Dubois, Phys. Fluids B, 590 (1993).

11. J. Moody developed a Transmitted Beam Diagnostic (TBD) at Lawrence Livermore National Laboratory to measure the angular distribution of the transmitted laser energy.

12. J. Murray, Lawrence Livermore National Laboratory, Livermore, CA, private communication (1995).

13. D. Pennington, et al., Tech. Dig. 8, 161 (1994).

14. S. Dixit, Lawrence Livermore National Laboratory, Livermore, CA, private communication (1995).

15. E. A. Williams, et al., Phys. Plasmas 2, 129 (1995).
16. H. A. Rose and D. F. Dubois, Phys. Rev. Lett. 72, 2883 (1994).

17. S. L. Wilks, et al., Phys. Rev. Lett. 74, 5048 (1995).

18. B. I. Cohen, et al., "Two-Dimensional Hybrid Simulations of Ponderomotively Driven Nonlinear Ion Waves," Lawrence Livermore National Laboratory, Livermore, CA, UCRL-JC121297 ABS; prepared for the 37th Annual Mtg. of APS Div. of Plasma Phys., Louisville, KY, Nov 6-10, 1995.

19. B. Ya Zel'dovich, N. F. Pipiletsky, and V. V. Shkunov, Principles of Phase Conjugation, Springer Series in Optical Sciences, T. Tamir, Ed. (Springer, Berlin, 1985), Vol. 42.

20. B. MacGowan, Lawrence Livermore National Laboratory, Livermore, CA, private communication (1995).

21. J. Fernandez, J. A. Cobble, B. H. Failor, W. W. Hsing, et al., "Dependence of SBS on Laser Intensity, Laser F Number and Ion Species in Hohlraum Plasmas," Los Alamos National Laboratory, Los Alamos, NM, LAUR-95-186; submitted to Phys. Rev. Lett.

22. B. B. Afeyan, Lawrence Livermore National Laboratory, Livermore, CA, private communication (1995).

23. C. Randall, 1978 Laser Program Annual Report 1, 3-53, Lawrence Livermore National Laboratory, Livermore, CA, UCRL-5002178 (1978).

24. C. Labaune, H. A. Baldis, N. Renard, E. Schifano, et al., Phys. Rev. Lett. 75(2), 248 (1995). 


\title{
FACILITY REPORT APRIL-JUNE 1995
}

\author{
G. Hermes
}

Nova Operations Group

During this quarter, Nova Operations fired a total of 313 system shots resulting in 337 experiments. These experiments were distributed among ICF experiments, Defense Sciences experiments, X-Ray Laser (XRL) experiments, Laser Sciences, and facility maintenance shots.

Work has begun in support of a series of XRL experiments planned for mid-August on Nova's 10-beam chamber. This effort includes planning and preparation to install cylinder lenses on beamline 3, the XRL assembly, the imaging diagnostics, and the alignment system.

The gated $x$-ray imager 4 was also completed, installed, and activated on Nova's 10-beam chamber.

We implemented a new "precision pulse shape" capability on Nova. This enables us to produce a power balance report immediately after each shot. Data from the $3 \omega$ streak camera and Incident Beam Diagnostic energy diodes are used to show plots of power vs time for the 10 beams and the power balance error vs time.
We began installation and activation of the 100-TW system with full-system activation planned for early fourth quarter. The compressor chamber was delivered and installed, and the 10-cm amplifier, spatial filters, other beamline components, and optical components for the compressor and diagnostics systems were installed and aligned. The vacuum system for the compressor chamber was completed. The control systems and interlock systems were installed and tested, including firing the $10-\mathrm{cm}$ amplifier.

Two Total Quality Management working groups were formed-one to focus on shot quality and the other on shot quantity. We have also made significant progress in cross training personnel among the operations groups. 


\section{Publications}

A

Afeyan, B. B., Berger, R. L., Bonnaud, G., Decker, C. D., Kruer, W. L., Langdon, A. B., Still, C. W., and Yang, T. B., Numerical Simulations of Stimulated Raman Scattering in Laser Hot Spots and Speckles, Lawrence Livermore National Laboratory, Livermore, CA, UCRL-JC-120886 ABS (1995). Prepared for 25th Annual Anomalous Absorption Conf, Aspen, CO, May 27-Jun 1, 1995.

Afeyan, B. B., Berger, R. L., Cohen, B. I., Kaiser, T. B., and Williams, E. A., Generalization of the MultiDimensional Theory of Stimulated Brillouin Scattering to Include Ponderomotive Filamentation, Lawrence Livermore National Laboratory, Livermore, CA, UCRL-JC-120768 ABS (1995). Prepared for 25th Annual Anomalous Absorption Conf, Aspen, CO, May 27-Jun 1, 1995.

Amendt, P., Glendinning, S. G., Hammel, B. A., Landen, O., Suter, L. J., Laffite, S., and Jadaud, J. P., Shocked Witness Foam-Ball Diagnostic for Nova Hohlraum Time-Dependent Drive Asymmetry, Lawrence Livermore National Laboratory, Livermore, CA, UCRL-JC-117033 ABS Rev. 1 (1995). Prepared for 25th Annual Anomalous Absorption Conf, Aspen, CO, May 27-Jun 1, 1995.

Amendt, P., Witness Foam-Ball Diagnostic for Nova Hohlraum Time-Dependent Drive Asymmetry, Lawrence Livermore National Laboratory, Livermore, CA, UCRL-JC-116322 (1995); Rev. Sci. Inst. 66(1), 785-787 (1995).

Azevedo, S. G., Brase, J. M., Mast, J. E., McEwan, T. E., and Twogood, R. E., Project Guardian, Lawrence Livermore National Laboratory, Livermore, CA, UCRL-ID-120802 (1995).
Azevedo, S. G., Gavel, D. T., Mast, J. E., and Warhus, J. P., Statement of Capabilities: Micropower Impulse Radar (MIR) Technology Applied to Mine Detection and Imaging, Lawrence Livermore National Laboratory, Livermore, CA, UCRL-ID-120801 (1995).

\section{B}

Back, C. A., Glenzer, S. H., Estabrook, K., MacGowan, B. J., Montgomery, D. S., Munro, D. H., Shepard, T. D., and Stone, G. F., Electron Temperature Scalings of Gasbag Targets, Lawrence Livermore National Laboratory, Livermore, CA, UCRL-JC-120783 ABS (1995). Prepared for 25th Annual Anomalous Absorption Conf, Aspen, CO, May 27-Jun 1, 1995.

Back, C., Characterization of Nova Plasmas Using an X-Ray Spectrometer with Temporal and Spatial Resolution, Lawrence Livermore National Laboratory, Livermore, CA, UCRL-JC-116211 (1995); Rev. Sci. Inst. 66(1), 764-766 (1995).

Barnard, J. J., Emittance Growth from Rotated Quadrupoles in Heavy Ion Accelerators, Lawrence Livermore National Laboratory, Livermore, CA, UCRL-JC-119385 (1995). Prepared for Institute for Electrical and Electronics Engineers (IEEE) 1995 Particle Accelerator Conf, Dallas, TX, May 1-5, 1995.

Beach, R. J., CW Theory of Quasi-Three Level EndPumped Laser Oscillators, Lawrence Livermore National Laboratory, Livermore, CA, UCRL-JC-121121 (1995). Submitted to IEEE J. Quantum Electron. 
Berger, R. L., Afeyan, B. B., Bonnaud, G., Langdon, A. B., Lasinski, B. F., Still, C. W., and Williams, E. A., Simulations of the Interaction of Filamentation with SRS and SBS, Lawrence Livermore National Laboratory, Livermore, CA, UCRL-JC-120773 ABS (1995). Prepared for 25th Annual Anomalous Absorption Conf, Aspen, CO, May 27-Jun 1, 1995.

Bibeau, C., Evaluation of the ${ }^{4} I_{11 / 2}$ Terminal Level Lifetime for Several Neodymium-Doped Laser Crystals and Glasses, Lawrence Livermore National Laboratory, Livermore, CA, UCRL-LR-120895 (1995).

Bibeau, C., Trenholme, J. B., and Payne, S. A., Pulse Length and Terminal Level Lifetime Dependence of Energy Extraction for Neodymium-Doped Phosphate Amplifier Glass, Lawrence Livermore National Laboratory, Livermore, CA, UCRL-JC-120909 (1995). Submitted to IEEE J. Quantum Electronics.

Bittner, D., Collins, G., Mapoles, E., and Bernat, T., Formation of Uniform Solid D2 Layers by IR Irradiation, Lawrence Livermore National Laboratory, Livermore, CA, UCRL-JC-121117 ABS (1995). Prepared for $42 n d$ Nat'l Symp of the American Vacuum Society, Minneapolis, $\mathrm{MN}$, Oct 16-20, 1995.

Bonnaud, G., Afeyan, B. B., Berger, R. L., Bouchut, F., Bouvet, C., Chiron, A., and Bers, A., Numerical Simulations of High-Frequency Parametric Instabilities, Brillouin Scattering and Beam Crossing in the Gas-Bag Experiment Context, Lawrence Livermore National Laboratory, Livermore, CA, UCRL-JC-120883 ABS (1995). Prepared for 25th Annual Anomalous Absorption Conf, Aspen, CO, May 27-Jun 1, 1995.

Boyd, R., High-Efficiency Metallic Diffraction Gratings for Laser Applications, Lawrence Livermore National Laboratory, Livermore, CA, UCRL-JC-115589 (1995); Applied Optics 34(4), 1697-1706 (1995).

Britten, J. A., Boyd, R. D., Perry, M. D., Shore, B. W., and Thomas, I. M., Low Efficiency Gratings for 3rd Harmonic Diagnostics Applications, Lawrence Livermore National Laboratory, Livermore, CA, UCRL-JC-120707 ABS (1995). Prepared for 1st Annual Int'l Conf on SolidState Lasers for Application to Inertial Confinement Fusion, Monterey, CA, May 30-Jun 2, 1995.

Brown, D. L., and Mannell, G. T., Thermal Analysis of the Large Close Packed Amplifiers in the National Ignition Facility (NIF), Lawrence Livermore National Laboratory, Livermore, CA, UCRL-JC-120211 (1995). Prepared for 1st Annual Int'l Conf on Solid-State Lasers for Application to Inertial Confinement Fusion, Monterey, CA, May 30-Jun 2, 1995.
Brusasco, R. M., Letts, S. A., Saculla, M. D., and Cook, R. C., High Beryllium Content Plasma Polymer Ablator Coatings for ICF Targets, Lawrence Livermore National Laboratory, Livermore, CA, UCRL-IC-120897 ABS (1995). Prepared for $42 n$ d Nat'l Symp of the American Vacuum Society, Minneapolis, MN, Oct 16-20, 1995.

\section{C}

Callahan, D. A., and Langdon, A. B., Transport of a Partially-Neutralized Ion Beam in a Heavy-Ion Fusion Reactor Chamber, Lawrence Livermore National Laboratory, Livermore, CA, UCRL-JC-119359 (1995). Prepared for IEEE 1995 Particle Accelerator Conf, Dallas, TX, May 1-5, 1995.

Callahan, D. A., Chamber Propagation Physics for Heavy Ion Fusion, Lawrence Livermore National Laboratory, Livermore, CA, UCRL-JC-121279 ABS (1995). Prepared for Int'l Symp on Heary Ion Inertial Fusion, Princeton, NJ, Sept 6-9, 1995.

Campbell, J. H., Barker, C. E., Van Wonterghem, B. M., Speck, D. R., Behrendt, W. C., Murray, J. R., Caird, J. A., Decker, D. E., and Smith, I. C., Performance Results for Beamlet: a Large Aperture, Multipass Nd:Glass Laser, Lawrence Livermore National Laboratory, Livermore, CA, UCRL-JC-120779 (1995). Prepared for Conf on Lasers and Electro Optics/Pacific Rim' 95, Chiba, Japan, Jul 11-14, 1995.

Campbell, J. H., Edwards, G., and Marion, J., Damage and Fracture in Large Aperture, Fused Silica, Vacuum Spatial Filter Lenses, Lawrence Livermore National Laboratory, Livermore, CA, UCRL-JC-120780 ABS (1995). Prepared for Ist Annual Int'l Conf on Solid-State Lasers for Application to Inertial Confinement Fusion, Monterey, CA, May 30-Jun 2, 1995.

Campbell, J., and Correll, D., ICF Quarterly Report, 5(1), Lawrence Livermore National Laboratory, Livermore, CA, UCRL-LR-105821-95-1 (1995).

Celliers, P., Da Silva, L., Mrowka, S., Dane, B., Norton, M., Matthews, D., Fiedorowicz, H., Bartnik, A., Abate, J., and Maldonado, J., Characterization and Optimization of Solid and Gas Target X-Ray Sources for Proximity Lithography, Lawrence Livermore National Laboratory, Livermore, CA, UCRL-JC-120781 ABS (1995). Prepared for ARPA Advanced Lithography Program Review, Scottsdale, AZ, Jan 23-27, 1995.

Celliers, P., Fringe Formation and Coherence of a Soft $X$-Ray Laser Beam Illuminating a Mach-Zehnder Interferometer, Lawrence Livermore National Laboratory, Livermore, CA, UCRL-JC-120921 (1995). Submitted to Optics Lett. (1995). 
Celliers, P., Weber, F., Da Silva, L. B., Barbee, T. W., Cauble, R., Wan, A. S., and Moreno, J. C., Fringe Formation and Coherence of a Soft X-Ray Laser Beam Illuminating a Mach-Zehnder Interferometer, Lawrence Livermore National Laboratory, Livermore, CA, UCRL-JC-120921 (1995). Submitted to Opt. Lett.

Chang, J. J., High-Power Copper Vapor Lasers and Applications, Lawrence Livermore National Laboratory, Livermore, CA, UCRL-JC-120599 ABS (1995). Prepared for NATO Advanced Research Workshop on Pulsed Metal Vapour Lasers, St Andrews, Scotland, Aug 7-10, 1995.

Cohen, B. I., Afeyan, B. B., Langdon, A. B., Lasinski, B. F., and Williams, E. A., Ion Wave Nonlinearities in Stimulated Brillouin Scattering, Lawrence Livermore National Laboratory, Livermore, CA, UCRL-JC-120774 ABS (1995). Prepared for 25th Annual Anomalous Absorption Conf, Aspen, CO, May 27-Jun 1, 1995.

Collins, G., Unites, W., Mapoles, E., and Bernat, T., Raman Study of Solid D-T, Lawrence Livermore National Laboratory, Livermore, CA, UCRL-JC-121118 ABS (1995). Prepared for 42nd Nat'l Symp of the American Vacuum Society, Minneapolis, MN, Oct 16-20, 1995.

Coverdale, C. A., Darrow, C. B., Decker, C. D., Mori, W. B., Tzeng, K.-C., Clayton, C. E., Marsh, K. A., and Joshi, C., Electron Acceleration in Relativistic Plasma Waves Generated by a Single-Frequency Short-Pulse Laser, Lawrence Livermore National Laboratory, Livermore, CA, UCRL-JC-120891 (1995). Prepared for IEEE 1995 Particle Accelerator Conf, Dallas, TX, May 1-5, 1995.

Coverdale, C. A., Darrow, C. B., Decker, C. D., Naumova, N., Bulanov, S., Mori, W. B., and Tzeng, K. C., Spectral Anomalies and Reflectivity Saturation of Stimulated Raman Backscatter from a Sub-Picosecond Laser in an Underdense Plasma, Lawrence Livermore National Laboratory, Livermore, CA, UCRL-JC-121142 (1995). Submitted to Phys. Rev. Lett.

Coverdale, C. A., The Interaction of Intense Subpicosecond Laser Pulses with Underdense Plasmas, Lawrence Livermore National Laboratory, Livermore, CA, UCRL-LR-120922 (1995).

\section{D}

Da Silva, L. B., Barbee, T. W., Cauble, R., Celliers, P., Ciarlo, D., Libby, S., London, R. A., Matthews, D., Mrowka, S., Moreno, J. C., Ress, D., Trebes, J. E., Wan, A. S., and Weber, F., Electron Density Measurements of High Density Plasmas Using Soft X-Ray Laser Interferometry, Lawrence Livermore National Laboratory, Livermore, CA, UCRL-JC-120243 (1995). Submitted to Phys. Rev. Lett.
Da Silva, L., Electron Density Measurements of High Density Plasmas Using Soft X-Ray Laser Interferometry, Lawrence Livermore National Laboratory, Livermore, CA, UCRL-JC-120243 (1995); Phys. Rev. Lett. 74(5), 3991-3994 (1995).

Da Silva, L., X-Ray Lasers for High Density Plasma Diagnostics, Lawrence Livermore National Laboratory, Livermore, CA, UCRL-JC-116411 (1995); Rev. Sci. Inst. 66(1), 574-578 (1995).

Dahlburg, J., Three Dimensional Multimode Simulations of the Ablative Rayleigh-Taylor Instability, Lawrence Livermore National Laboratory, Livermore, CA, UCRL-JC-118845 (1995); Phys. Plasmas 2(6), 2453-2459 (1995).

Darrow, C., Hay, G., Zakharenkov, Y., Nguyen, H., Hammel, B., and Perry, M. D., Experimental Investigation of the Interaction of $1 \mu \mathrm{m}, 0.7 \mathrm{ps}, 10^{19} \mathrm{~W} / \mathrm{cm}^{2}$ Laser Pulses with Solid Targets, Lawrence Livermore National Laboratory, Livermore, CA, UCRL-JC-120245 ABS (1995). Prepared for 25th Annual Anomalous Absorption Conf, Aspen, CO, May 27-Jun 1, 1995.

De Yoreo, J. J., Rek, Z. U., Zaitseva, N. P., Woods, B. W., and Land, T. A., Sources of Strain in Rapidly Grown Crystals of $\mathrm{KH}_{2} \mathrm{PO}_{4}$, Lawrence Livermore National Laboratory, Livermore, CA, UCRL-JC-119645 (1995). Prepared for Int'l Conf on Crystal Growth XI, The Hague, Netherlands, Jun 18-23, 1995.

De Yoreo, J. J., Rek, Z., Zaitseva, N. P., Torres, R., Lee, J. D., and Woods, B. W., The Dependence of Optical Homogeneity on Growth Rate in $\mathrm{KH}_{2} \mathrm{PO}_{4}$, Lawrence Livermore National Laboratory, Livermore, CA, UCRL-JC-120235 ABS (1995). Prepared for 14th Conf on Crystal Growth and Epitaxy, Fallen Leaf Lake, CA, Jun 4-7, 1995.

De Yoreo, J. J., Zaitseva, N. P., Rek, Z. U., Land, T. A., and Woods, B. W., The Connection between Optical Distortion and Elemental Growth Processes in RapidlyGrown, Single Crystals of $\mathrm{KH}_{2} \mathrm{PO}_{4}$, Lawrence Livermore National Laboratory, Livermore, CA, UCRL-JC-120701 (1995). Submitted to Appl. Phys. Lett.

Deadrick, F. J., Barnard, J. J., Fessenden, T. J., Meridith, J. W., and Rintamaki, J., Development of Beam Position Monitors for Heavy Ion Recirculators, Lawrence Livermore National Laboratory, Livermore, CA, UCRL-JC-119539 (1995). Prepared for IEEE 1995 Particle Accelerator Conf, Dallas, TX, May 1-5, 1995. 
Decker, C. D., Eder, D. C., and London, R. A., Ionization Induced Refraction in Recombination X-Ray Lasers, Lawrence Livermore National Laboratory, Livermore, CA, UCRL-JC-119324 (1995). Submitted to Physics of Plasmas.

DeLoach, L. D., Page, R. H., Wilke, G. D., Payne, S. A., and Krupke, W. F., Transition Metal-Doped Zinc

Chalcogenides: Spectroscopy and Laser Demonstration of a New Class of Gain Media, Lawrence Livermore National Laboratory, Livermore, CA, UCRL-JC-120816 (1995).

Submitted to IEEE J. Quantum Electron.

Dendooven, P., A Freon-Filled, Holographic Bubble Chamber as a High-Energy Photon Burst Spectrometer, Lawrence Livermore National Laboratory, Livermore, CA, UCRL-JC-116184 (1995); Rev. Sci. Inst. 66(1), 571-573 (1995).

Ditmire, T., Amplification of XUV Harmonic Radiation in a Gallium Amplifier, Lawrence Livermore National Laboratory, Livermore, CA, UCRL-JC-119550 (1995); Phys. Rev. A51(6), R4337-R4340 (1995).

Ditmire, T., Calculation and Measurement of High Order Harmonic Energy Yields in Helium, Lawrence Livermore National Laboratory, Livermore, CA, UCRL-JC-121469 (1995). Submitted to J. Opt. Soc. Am. B (1995).

Ditmire, T., Energy Yield and Conversion Efficiency Measurements of High Order Harmonic Radiation. Lawrence Livermore National Laboratory, Livermore, CA, UCRL-JC-118146 (1995); Phys. Rev. A51(2), R902-R905 (1995).

\section{E}

Ehrlich, R. B., Miller, J. L., Saunders, R. L., Thompson, C. E., Weiland, T. L., and Laumann, C. W., Precision Nova Operations, Lawrence Livermore National Laboratory, Livermore, CA, UCRL-JC-120175 (1995). Prepared for 1st Annual Int'l Conf on Solid-State Lasers for Application to Inertial Confinement Fusion, Monterey, CA, May 30-Jun 2, 1995.

English, R. E., Trip Report-Assessment of CILAS Interferometer, Lawrence Livermore National Laboratory, Livermore, CA, UCRL-ID-120250 (1995).

\section{$\mathbf{F}$}

Fessenden, T. J., Grote, D. P., and Sharp, W. M., Accelerator Waveform Synthesis and Longitudinal Beam Dynamics in a Small Induction Recirculator, Lawrence Livermore National Laboratory, Livermore, CA, UCRL-JC-119580 (1995). Prepared for IEEE 1995 Particle Accelerator Conf, Dallas, TX, May 1-5, 1995.
G

Galakhov, I. V., Gruzin, I. A., Gudov, S. N., Kirillov, G. A., Logutenko, S. L., Murugov, V. M., Osin, V. A., and Zolotovskii, V. I., Final Report Task Order No. B23964I between Regents of the University of California and Institute of Experimental Physics Task 2: Switch Development, Lawrence Livermore National Laboratory, Livermore, CA, UCRL-CR-120302 (1995), p 2.

Galakhov, I. V., Kirillov, G. A., and Murugov, V. M., Final Report Task Order No. $B 239641$ between Regents of the University of California and Institute of Experimental Physics Task 1: Conceptual Design, Lawrence Livermore National Laboratory, Livermore, CA, UCRL-CR-120302 (1995), p 1.

Gavel, D. T., Mast, J. E., Warhus, J., and Azevedo, S. G., An Impulse Radar Array for Detecting Land Mines, Lawrence Livermore National Laboratory, Livermore, CA, UCRL-JC-120550 (1995). Prepared for Autonomous Vehicles in Mine Countermeasures, Naval Postgraduate School, Monterey, CA, Apr 4-7, 1995.

Glinsky, M. E., Bailey, D., Belak, J., Eder, D., London, R. A., Zimmerman, G. B., Antoun, T., Curran, D. R., Seaman, L., and Jacques, S. L., Medical Applications of Stress Wave Propagation and Material Failure Physics, Lawrence Livermore National Laboratory, Livermore, CA, UCRL-JC-121278 ABS (1995). Prepared for American Physical Society Topical Conf, Seattle, WA, Aug 13-16, 1995.

Glinsky, M. E., London, R. A., Zimmerman, G. B., and Jacques, S. L., Computer Modeling of Endovascular Patch Welding Using Temperature Feedback, Lawrence Livermore National Laboratory, Livermore, CA, UCRL-JC-120751 ABS (1995). Prepared for BiOS Europe '95, Barcelona, Spain, Sept 12-16, 1995.

Glinsky, M., Regimes of Suprathermal Electron Transport, Lawrence Livermore National Laboratory, Livermore, CA, UCRL-JC-118514 (1995); Phys. Plasmas 2(7), 2796-2806 (1995).

Green, M., Peterson, N., Sato, T., Teramoto, A., Cook, R., and Lifson, S., A Helical Polymer with a Cooperative Response to Chiral Information, Lawrence Livermore National Laboratory, Livermore, CA, UCRL-JC-121098 (1995); Science 268, 1860-1866 (1995).

Grote, D. P., Friedman, A., and Haber, I., Three Dimensional Simulations of a Small Induction Recirculator Accelerator, Lawrence Livermore National Laboratory, Livermore, CA, UCRL-JC-119579 (1995). Prepared for IEEE 1995 Particle Accelerator Conf, Dallas, TX, May 1-5, 1995. 
Grote, D. P., Friedman, A., Haber, I., and Yu, S., ThreeDimensional Simulations of High-Current Beams in Induction Accelerators with WARP3D, Lawrence Livermore National Laboratory, Livermore, CA, UCRL-JC-121280 ABS (1995). Prepared for Int'l Symp on Heavy Ion Inertial Fusion, Princeton, NJ, Sept 6-9, 1995.

\section{$\mathbf{H}$}

Haan, S. W., Strobel, G. L., Pollaine, S. M., and Marinak, M. M., Hydrodynamic Instability Modeling on Ignition ICF Capsules, Lawrence Livermore National Laboratory, Livermore, CA, UCRL-JC-120888 ABS (1995). Prepared for 5th Int'l Workshop on the Physics of Compressible Turbulent Mixing, Stonybrook, NY, Jul 18-21, 1995.

Haan, S., Design and Modeling of Ignition Targets for the National Ignition Facility, Lawrence Livermore National Laboratory, Livermore, CA, UCRL-JC-117034 (1995); Phys. Plasmas 2(6), 2480-2487 (1995).

Hinkel, D. E., Williams, E. A., Berger, R. L., and Powers, L. V., Beam Steering Induced by Transverse Plasma Flow, Lawrence Livermore National Laboratory, Livermore, CA, UCRL-JC-120771 ABS (1995). Prepared for 25th Annual Anomalous Absorption Conf, Aspen, CO, May 27-Jun 1, 1995.

Honea, E. C., Collins, G. W., McEachern, R. L., and Wallace, R. J., Surface Roughness Scaling in SputterDeposited Beryllium and Beryllium-Boron Films, Lawrence Livermore National Laboratory, Livermore, CA, UCRL-JC-121145 ABS (1995). Prepared for Material Research Society 1995 Fall Mtg, Boston, MA, Nov 27-Dec 1, 1995.

Hsieh, E. J., Behymer, R. D., Greenfield, B. J., and Lindsey, E. F., Properties of Be Films Sputter Coated onto Hemispherical Substrates under Various Conditions Including Gas Pulsing, Lawrence Livermore National Laboratory, Livermore, CA, UCRL-JC-120892 ABS (1995). Prepared for 42nd Nat'l Symp of the American Vacuum Society, Minneapolis, MN, Oct 16-20, 1995.

J

Johnson, R., Laser Fusion Efforts in the United States, Lawrence Livermore National Laboratory, Livermore, CA, UCRL-JC-117893 (1995); Optics \& Photonics News 6, 16-23 (1995).

Judd, D. L., Rintamaki, J., Lund, S. M., Barnard, J. J., Fessenden, T., Grote, D. P., and Deadrick, F. J., Heavy Ion Fusion Notes 94-1 through 94-9, Lawrence Livermore National Laboratory, Livermore, CA, UCRL-ID-120197 (1995).
K

Kalantar, D. H., Da Silva, L. B., Glendinning, S. G., Key, M. H., Weber, F., Weber, S. V., and Knauer, J. P., $\mathrm{X}$-Ray Laser Backlighting Applied to Measure Laser Imprint, Lawrence Livermore National Laboratory, Livermore, CA, UCRL-JC-120244 ABS (1995). Prepared for 25th Annual Anomalous Absorption Conf, Aspen, CO, May 27-Jun 1, 1995.

Kalantar, D., X-Ray Imaging of Uniform Large ScaleLength Plasmas Created from Gas-Filled Targets on Nova, Lawrence Livermore National Laboratory, Livermore, CA, UCRL-JC-116195 (1995); Rev. Sci. Inst. 66(1), $782-784$ (1995).

Kauffman, R., Drive Characterization of Indirect Drive Targets on the Nova Laser, Lawrence Livermore National Laboratory, Livermore, CA, UCRL-JC-116765 (1995); Rev. Sci. Inst. 66(1), 678-682 (1995).

Keane, C. J., Landen, O. L., Hammel, B. A., Cable, M., Cook, R., Dittrich, T., Haan, S., Hatchett, S. P., McEachern, R., Murphy, T., Nelson, M., Suter, L., Wallace, R., Chrien, R., Colvin, J., Hoffman, N., Hsing, W., and Pollak, G., Nova High Growth Factor Implosion Experiments and Analysis, Lawrence Livermore National Laboratory, Livermore, CA, UCRL-JC-120800 ABS (1995). Prepared for 25 in Annual Anomalous Absorption Conf, Aspen, CO, May 27-Jun 1, 1995.

Keane, C., Diagnosis of Pusher-Fuel Mix in Spherical Implosions Using X-Ray Spectroscopy, Lawrence Livermore National Laboratory, Livermore, CA, UCRL-JC-116787 (1995); Rev. Sci. Inst. 66(1), 689-696 (1995).

Key, M. H., Barbee, T. W., Da Silva, L. B., Glendinning, S. G., Kalantar, D. H., Rose, S. J., and Weber, S. V., New Plasma Diagnostic Possibilities from Radiography with XUV Lasers, Lawrence Livermore National Laboratory, Livermore, CA, UCRL-JC-118848 (1995). Submitted to J. Quant. Spectros. Radiat. Transfer.

Kirkwood, R. K., Afeyan, B. B., Kruer, W. L., MacGowan, B. J., Montgomery, D. S., Moody, J. D., and Wilks, S. C., Observation of Energy Transfer between Frequency Mismatched Laser Beams in a Large Scale Plasma, Lawrence Livermore National Laboratory, Livermore, CA, UCRL-JC-120249 ABS (1995). Prepared. for 25th Annual Anomalous Absorption Conf, Aspen, CO, May 27-Jun 1, 1995 
Kirkwood, R. K., Back, C. A., Estabrook, K. G., Kalantar, D. H., MacGowan, B. J., Montgomery, D. S., and Moody, J. D., Imaging of Near to Back Scattered Light in Gas Target Plasmas, Lawrence Livermore National Laboratory, Livermore, CA, UCRL-JC-120248 ABS (1995). Prepared for 25th Annual Anomalous Absorption Conf, Aspen, CO, May 27-Jun 1, 1995.

Kruer, W. L., and Wilks, S. C., Higher Temperature Hohlraums Using Laser Beam Smoothing, Lawrence Livermore National Laboratory, Livermore, CA, UCRL-JC-121287 ABS (1995). Prepared for 37th Annual Meeting of the American Physical Society Division of Plasma Physics, Louisville, KY, Nov 6-10, 1995.

Kruer, W. L., and Wilks, S. C., Novel Techniques to Control Stimulated Raman and Brillouin Scattering in Laser Plasmas, Lawrence Livermore National Laboratory, Livermore, CA, UCRL-JC-120876 ABS (1995). Prepared for 25th Annual Anomalous Absorption Conf, Aspen, CO, May 27-Jun 1, 1995.

Kruer, W. L., Wilks, S. C., Afeyan, B. B., and Kirkwood, R. K., Energy Transfer between Crossing Laser Beams, Lawrence Livermore National Laboratory, Livermore, CA, UCRL-JC-120776 (1995). Submitted to Physics of Plasmas.

Kruer, W. L., Wilks, S. C., Kirkwood, R., and Afeyan, B., Energy Transfer between Crossing Laser Beams, Lawrence Livermore National Laboratory, Livermore, CA, UCRL-JC-120776 ABS (1995). Prepared for 25th Annual Anomalous Absorption Conf, Aspen, CO, May 27-Jun 1, 1995.

\section{L}

Land, T. A., De Yoreo, J. J., and Lee, J. D., Growth Morphology of Vicinal Hillocks on the (101) Face of $\mathrm{KH}_{2} \mathrm{PO}_{4}$ : Evidence of Surface Diffusion, Lawrence Livermore National Laboratory, Livermore, CA, UCRL-JC-119608 ABS (1995). Prepared for Materials Research Society Conf, Boston, MA, Nov 27-Dec 1, 1995.

Land, T. A., De Yoreo, J. J., Malkin, A. J., Lee, J. D., Kuznetsov, Yu. G., and McPherson, A., Growth Mechanisms and Morphologies of Solution-Based Crystals: an AFM Perspective - II, Lawrence Livermore National Laboratory, Livermore, CA, UCRL-JC-120239 ABS (1995). Prepared for 14th Confon Crystal Growth and Epitaxy, Fallen Leaf Lake, CA, Jun 4-7, 1995.
Land, T. A., Malkin, A., Kuznetsov, Y. G., De Yoreo, J. J., and McPherson, A., Investigation of Canavalin Crystallization Using In-Situ Atomic Force Microscopy, Lawrence Livermore National Laboratory, Livermore, CA, UCRL-JC-121141 ABS (1995). Prepared for 6th Int'l Conf on Crystallization of Biological Macromolecules (ICCBM-6), Hiroshima, Japan, Nov 12-17, 1995.

Landen, O., An X-Ray Technique for Precision Laser Beam Synchronization, Lawrence Livermore National Laboratory, Livermore, CA, UCRL-JC-115599 (1995); Rev. Sci. Inst. 66(1), 788-790 (1995).

Landen, O., Indirectly Driven, High Growth Rayleigh-Taylor Implosions on Nova, Lawrence Livermore National Laboratory, Livermore, CA, UCRL-JC-120196 (1995). Submitted to J. Quant. Spect. (1995).

Langer, S. H., Keane, C. J., and Scott, H. A., TwoDimensional Models of Emission Line Ratios in ICF Target Implosions, Lawrence Livermore National Laboratory, Livermore, CA, UCRL-JC-120788 ABS (1995). Prepared for 25th Annual Anomalous Absorption Conf, Aspen, CO, May 27-Jun 1, 1995.

Larson, D. W., Final Report for 1.7 Megajoule Prototype Bank Testing, Lawrence Livermore National Laboratory, Livermore, CA, UCRL-CR-120303 (1995).

Lasinski, B. F., Afeyan, B. B., Cohen, B. I., Langdon, A. B., and Williams, E. A., Ion Wave Modeling in PIC-Fluid Simulations, Lawrence Livermore National Laboratory, Livermore, CA, UCRL-JC-120775 ABS (1995). Prepared for 25th Annual Anomalous Absorption Conf, Aspen, CO, May 27-Jun 1, 1995.

Lawson, J. K., Wolfe, C. R., Manes, K. R., Trenholme, J. B., Aikens, D. M., and English, R. E., Specification of Optical Components Using the Power Spectral Density Function, Lawrence Livermore National Laboratory, Livermore, CA, UCRL-JC-120920 (1995). Prepared for Society of Photo-Optical Instrumentation Engineers' 40 th Annual Mtg, San Diego, CA, Jul 9-14, 1995.

Lawson, J., Simulation of Phase Structure, Lawrence Livermore National Laboratory, Livermore, CA, UCRL-ID-120900 (1995).

Lerche, R. A., Cable, M. D., and Dendooven, P. G., ICF Burn-History Measurements Using 17-MeV Fusion Gamma Rays, Lawrence Livermore National Laboratory, Livermore, CA, UCRL-JC-119537 (1995). Prepared for 12th Int'l Conf on Laser Interaction and Related Plasma Phenomena, Osaka, Japan, Apr 24-28, 1995. 
Lerche, R. A., Cable, M. D., Hatchett, S. P., Caird, J. A., Kilkenny, J. D., Kornblum, H. N., Lane, S. M., Laumann, C., Murphy, T. J., Murray, J., Nelson, M. B., Phillion, D. W., Powell, H., and Ress, D., High Convergence, Indirect Drive Inertial Confinement Fusion Experiments at Nova, Lawrence Livermore National Laboratory, Livermore, CA, UCRL-JC-119536 (1995). Prepared for 12th Int'l Conf on Laser Interaction and Related Plasma Phenomena, Osaka, Japan, Apr 24-28, 1995.

Lerche, R., 25 ps Neutron Detector for Measuring ICFTarget Burn History, Lawrence Livermore National Laboratory, Livermore, CA, UCRL-JC-116183 (1995); Rev. Sci. Inst. 66(1), 933-935 (1995).

Letts, S. A., Fearon, E. M., Buckley, S. R., Saculla, M. D., Allison, L. M., and Cook, R., Fabrication of Special ICF Targets Using a Depolymerizable Mandrel Technique, Lawrence Livermore National Laboratory, Livermore, CA, UCRL-JC-121115 ABS (1995). Prepared for 42nd Nat'l Symp of the American Vacuum Society, Minneapolis, MN, Oct 16-20, 1995.

Letts, S., Fearon, E., Allison, L., Buckley, S., Saculla, M., and Cook, R., Decomposable Mandrel Project-Progress Report, Lawrence Livermore National Laboratory, Livermore, CA, UCRL-ID-120913 (1995).

Lindl, J. D., Target Physics Basis for Indirect Drive Targets for Inertial Fusion Energy, Lawrence Livermore National Laboratory, Livermore, CA, UCRL-JC-121143 ABS (1995). Prepared for Int'l Symp on Heavy Ion Inertial Fusion, Princeton, NJ, Sept 6-9, 1995.

Lindl, J., and Correll, D., 1994 ICF Annual Report, Lawrence Livermore National Laboratory, Livermore, CA, UCRL-LR-105820-94 (1995).

Logan, B. G., Relevance of the U.S. National Ignition Facility for Driver and Target Options to Next-Step Inertial Fusion Test Facilities, Lawrence Livermore National Laboratory, Livermore, CA, UCRL-JC-120766 (1995). Prepared for 12th Int' $l$ Conf on Laser Interaction and Related Plasma Phenomena, Osaka, Japan, Apr 24-28, 1995.

Logan, B. G., The NIF and Its Role in Inertial Fusion Energy, Lawrence Livermore National Laboratory, Livermore, CA, UCRL-ID-120777 (1995).

Logan, G., and Tobin, M., White Paper: Laser System and Target Chamber Design Needs for Inertial Fusion Energy Experiments in the National Ignition Facility, Lawrence Livermore National Laboratory, Livemore, CA, UCRLAR-120757 (1995).

\section{$\mathbf{M}$}

MacGowan, B. J., The Study of Parametric Instabilities in Large-Scale-Length Plasmas on Nowa, Lawrence Livermore National Laboratory, Livermore, CA, UCRL-JC-120911 ABS (1995). Prepared for 25th Annual Anomalous Absorption Conf, Aspen, CO, May 27-Jun 1, 1995.

Mapoles, E. R., Pipes, J. W., Ellefson, R. E., and Sowders, M. H., A Self-Contained Deuterium-Tritium Source for Inertial Fusion Target Development, Lawrence Livermore National Laboratory, Livermore, CA, UCRL-JC-121116 ABS (1995). Prepared for 42nd Nat'l Symp of the American Vacuum Society, Minneapolis, MN, Oct 16-20, 1995.

Marshall, C. D., Payne, S. A., Speth, J. A., Krupke, W. F., Quarles, G. J., Castillo, V., and Chai, B. H. T., Ultraviolet Laser Emission Properties of Ce:LiSrAlF ${ }_{6}$ and Ce:LiCaAlF ${ }_{6}$ Lawrence Livermore National Laboratory, Livermore, CA, UCRL-JC-120814 ABS (1995). Prepared for Optical Society of America Annual Mtg, Portland, OR, Sept 10-15,1995.

McEwan, T. E., Micropower Impulse Radar Technical Information, Lawrence Livermore National Laboratory, Livermore, CA, UCRL-ID-120903 (1995).

Molau, N. E., Genin, F. Y., Shang, C. C., and Kozlowski, M. R., Electro-Mechanical Modeling of Nodular Defects in Multilayer Optical Coatings, Lawrence Livermore National Laboratory, Livermore, CA, UCRL-JC-121308 ABS (1995). Prepared for 1st Biennial Tri-Laboratory Engineering Conf on Computational Modeling, Pleasanton, CA, Oct 31-Nov 2, 1995.

Montesanti, R. C., Thompson, S. L., Latanich, L. L., and Marion, J. E., Fabrication of Large Potassium Di-Hydrogen Phosphate (KDP) Crystals for the National Ignition Facility, Lawrence Livermore National Laboratory, Livermore, CA, UCRL-JC-120713 ABS (1995). Prepared for 1st Annual Int'l Conf on Solid-State Lasers for Application to Inertial Confinement Fusion, Monterey, CA, May 30-Jun 2, 1995.

Montgomery, D. S., Kirkwood, R. K., MacGowan, B. J., Moody, J. C., Back, C. A., Glenzer, S. H., Kalantar, D. H., Afeyan, B. B., Berger, R. L., Munro, D. H., Desenne, D., Richard, A., and Rousseaux, C., Angular Distribution of SRS from Nova Gasbag Plasmas, Lawrence Livermore National Laboratory, Livermore, CA, UCRL-JC-120246 ABS (1995). Prepared for 25th Annual Anomalous Absorption Conf, Aspen, CO, May 27-Jun 1, 1995. 
Moody, J. D., MacGowan, B. J., Kirkwood, R. K., Montgomery, D. S., Berger, R. L., Hinkel, D. E., Shepard, T. D., and Williams, E. A., Whole Beam Deflection through a Hohlraum Window Plasma, Lawrence Livermore National Laboratory, Livermore, CA, UCRL-JC-120915 ABS (1995). Prepared for 25th Annual Anomalous Absorption Conf, Aspen, CO, May 27-Jun 1, 1995.

Moody, J. D., MacGowan, B. J., Kirkwood, R. K., Montgomery, D. S., Desenne, D., Rousseaux, C., Berger, R. L., Kalantar, D. H., and Munro, D., Propagation of High Intensity Lasers through Large Scalelength Plasmas, Lawrence Livermore National Laboratory, Livermore, CA, UCRL-JC-120247 ABS (1995). Prepared for 25th Annual Anomalous Absorption Conf, Aspen, CO, May 27-Jun 1, 1995.

Morales, R., High Precision Wolter Optic Calibration Facility, Lawrence Livermore National Laboratory, Livermore, CA, UCRL-JC-116181 (1995); Rev. Sci. Inst. 66(1), 700-702 (1995).

Murphy, T. J., Delamater, N. D., Richard, A. L., Cable, M. D., Chrien, R., Gifford, K., Hauer, A. A., Lindman, E. L., Magelssen, G. R., Moore, J. B., Nelson, M. B., Pollaine, S. M., Powers, L. V., Suter, L. J., Wallace, R. J., and Wilde, B. H., Results from Implosion Experiments with Gas-Filled Nova Hohlraums, Lawrence Livermore National Laboratory, Livermore, CA, UCRL-JC-120761 ABS (1995). Prepared for 25th Annual Anomalous Absorption Conf, Aspen, CO, May 27-Jun 1, 1995.

Murphy, T. J., Recent Implosion Experiments on Nova, Lawrence Livermore National Laboratory, Livermore, CA, UCRL-JC-120918 ABS (1995). Prepared for 16th IEEE /NPSS, Champaign, IL, Sept 30-Oct 5, 1995.

Murphy, T., Ion-Temperature Measurement of Indirectly Driven Implosions Using a Geometry-Compensated Neutron Time-of-Flight Detector, Lawrence Livermore National Laboratory, Livermore, CA, UCRL-JC-116199 (1995); Rev. Sci. Inst. 66(1), 930-932 (1995).

Murray, J., and Correll, D., ICF Quarterly Report, 5(2), Lawrence Livermore National Laboratory, Livermore, CA, UCRL-LR-105821-95-2 (1995).
O

Orth, C. D., and Payne, S. A., System Study of a DiodePumped Solid-State-Laser Driver for Inertial Fusion Energy, Lawrence Livermore National Laboratory, Livermore, CA, UCRL-JC-120221 (1995). Prepared for 1st Annual Int'l Conf on Solid-State Lasers for Application to Inertial Confinement Fusion, Monterey, CA, May 30-Jun 2, 1995.

Orzechowski, T. J., Kauffman, R. L., Kirkwood, R. K., Kornblum, H. N., Levedahl, W. K., Montgomery, D. S., Powers, L. V., Shepard, T. D., Stone, G. F., Suter, L. J., Wallace, R. J., Foster, J. M., and Rosen, P., Dynamics of Gas-Filled Hohlraums, Lawrence Livermore National Laboratory, Livermore, CA, UCRL-JC-119372 (1995). Prepared for 12 Int'l Conf on Laser Interaction and Related Plasma Phenomena, Osaka, Japan, Apr 24-28, 1995.

Orzechowski, T. J., Kauffman, R. L., Kirkwood, R. K., Kornblum, H. N., Montgomery, D. S., Powers, L. V., Stone, G. F., Suter, L. J., and Wallace, R. J., Energetics of Gas-Filled Hohlraums, Lawrence Livermore National Laboratory, Livermore, CA, UCRL-JC-120760 ABS (1995). Prepared for 25th Annual Anomalous Absorption Conf, Aspen, CO, May 27-Jun 1, 1995.

Overturf, G. E., Buckley, S. R., Letts, S. A., Wilemski, G., Cook, R., McClellan, M. R., and Schroen-Carey, D., Low Density Resorcinol-Formaldehyde Aerogel Foam Shells for Use as Cryogenic ICF Experiments, Lawrence Livermore National Laboratory, Livermore, CA, UCRL-JC-120898 ABS (1995). Prepared for 42nd Nat'l Symp of the American Vacuum Society, Minneapolis, MN, Oct 16-20, 1995.

\section{$\mathbf{P}$}

Page, R. H., DeLoach, L. D., Wilke, G. D., Payne, S. A., Beach, R. J., and Krupke, W. F., Cr ${ }^{2+}$-Doped II-VI Crystals: New Widely-Tunable, Room-Temperature Mid-IR Lasers, Lawrence Livermore National Laboratory, Livermore, CA, UCRL-JC-120815 ABS (1995). Prepared for Lasers and Electro Optics Society '95 8th Annual Mtg, San Francisco, CA, Oct 30-Nov 2, 1995.

Payne, S. A., Marshall, C. D., Bayramian, A. J., Wilke, G. D., and Hayden, J. S., Properties of a New Average Power Nd-Doped Phosphate Laser Glass, Lawrence Livermore National Laboratory, Livermore, CA, UCRL-JC-118191 (1995). Prepared for Advanced Solid State Lasers 10th Topical Mtg, Memphis, TN, Jan 30-Feb 2, 1995.

Payne, S. A., Powell, H. T., and Krupke, W. F., Can Solid-State Laser Technology Serve Usefully beyond Fusion Ignition Facilities? Lawrence Livermore National Laboratory, Livermore, CA, UCRL-JC-120887 ABS (1995). Prepared for 1st Annual Int'l Conf on Solid-State Lasers for Application to Inertial Confinement Fusion, Monterey, CA, May 30-Jun 2, 1995. 
Payne, S., Cross Sections and Quantum Yields of the $3 \mu \mathrm{m}$ Emission for $\mathrm{Er}^{3+}$ and $\mathrm{Ho}^{3+}$ Dopants in Crystals, Lawrence Livermore National Laboratory, Livermore, CA, UCRL-JC-117882 (1995); J. Appl. Phys. 77(5), 4274-4279 (1995).

Pennington, D. M., Henesian, M. A., Wilcox, R. B., Weiland, R. B., Eimerl, D., Ehrlich, R. B., Laumann, C. W. and Miller, J. L., A Four-Color Beam Smoothing Irradiation System for Laser-Plasma Interaction Experiments at LLNL, Lawrence Livermore National Laboratory, Livermore, CA, UCRL-JC-121270 (1995). Prepared for 1st Annual Int'l Conf on Solid-State Lasers for Application to Inertial Confinement Fusion, Monterey, CA, May 30-Jun 2, 1995.

Perry, M. D., Dixit, S. N., Shore, B. W., Boyd, R. D., Britten, J. A., and Powell, H. T., High Efficiency Gratings for Beam Steering on the National Ignition Facility (NIF) Laser, Lawrence Livermore National Laboratory, Livermore, CA, UCRL-JC-120185 (1995). Prepared for 1st Annual Int'l Conf on Solid-State Lasers for Application to Inertial Confinement Fusion, Monterey, CA, May 30-Jun 2, 1995.

Pollaine, S. M., Haan, S. W., Amendt, P. A., Moreno, J. C., and Wan, A. S., Robustness Studies and Beam Angles for the National Ignition Facility, Lawrence Livermore National Laboratory, Livermore, CA, UCRL-JC-120785 ABS (1995). Prepared for 25th Annual Anomalous Absorption Conf, Aspen, CO, May 27-Jun 1, 1995.

Pollaine, S. M., Suter, L. J., Powers, L. V., Kauffman, R. L., Murphy, T. J., and Shepard, T. D., Gas Hohlraum Simulations, Lawrence Livermore National Laboratory, Livermore, CA, UCRL-JC-120786 ABS (1995). Prepared for 25th Annual Anomalous Absorption Conf, Aspen, CO, May 27-Jun 1, 1995.

Powers, L. V., Suter, L. J., Glendinning, S. G., Kauffman, R. L., Kirkwood, R., Montgomery, D. S., Murphy, T. J., Orzechowski, T. J., Pollaine, S. M., Ress, D. B., Shepard, T. D., Delamater, N. D., Hauer, A. A., Lindman, E. L., and Magelssen, G. R., Analysis of Effect of Gas Fills on Nova-Scale Hohlraum Dynamics and Energetics, Lawrence Livermore National Laboratory, Livermore, CA, UCRL-JC-120789 ABS (1995). Prepared for 25th Annual Anomalous Absorption Conf, Aspen, CO, May 27-Jun 1, 1995.

Powers, L., Low Stimulated Brillouin Backscatter Observed from Large, Hot Plasmas in Gas-Filled Hohlraums, Lawrence Livermore National Laboratory, Livermore, CA, UCRL-JC-118394 (1995); Phys. Rev. Lett. 74(4), 2957-2960 (1995).

\section{$\mathbf{R}$}

Remington, B. A., Marinak, M. M., Weber, S. V., Budil, K. S., Landen, O. L., Glendinning, S. G., Haan, S. W., Kilkenny, J. D., Wallace, R. J., and Dimonte, G., 2D and 3D Ablation Front Hydrodynamic Instability Experiments on Nova, Lawrence Livermore National Laboratory, Livermore, CA, UCRL-JC-119388 (1995). Prepared for 12th Int'l Conf on Laser Interaction and Related Plasma Phenomena, Osaka, Japan, Apr 24-28, 1995.

Remington, B. A., Marinak, M. M., Weber, S. V., Budil, K. S., Landen, O. L., Haan, S. W., Kilkenny, J. D., and Wallace, R. J., Single-Mode Rayleigh-Taylor Experiments in $2 D$ and $3 D$, Lawrence Livermore National Laboratory, Livermore, CA, UCRL-JC-120906 ABS (1995). Prepared for 5th Int'l Workshop on the Physics of Compressible Turbulent Mixing, Stonybrook, NY, Jul 18-21, 1995.

Remington, B., Single-Mode and Multimode Rayleigh-Taylor Experiments, Lawrence Livermore National Laboratory, Livermore, CA, UCRL-JC-116897 (1995); Phys. Plasmas 2(1), 241-255 (1995).

\section{$\mathrm{S}$}

Sheehan, L., Kozlowski, M., and Trench, B., Full Aperture Laser Conditioning of Multilayer and Polarizers, Lawrence Livermore National Laboratory, Livermore, CA, UCRL-JC-120203 (1995). Prepared for 1st Annual Int'l Conf on Solid-State Lasers for Application to Inertial Confinement Fusion, Monterey, CA, May 30-Jun 2, 1995.

Shepard, T. D., Orzechowski, T. J., Murphy, T. J., Suter, L. J., Delamater, N. D., Richard, A. L., Foster, J. M., and Rosen, P. A., Interface-Motion Modeling for Nova Gas Hohlraums, Lawrence Livermore National Laboratory, Livermore, CA, UCRL-JC-120787 ABS (1995). Prepared for 25th Annual Anomalous Absorption Conf, Aspen, CO, May 27-Jun 1, 1995.

Shepard, T., Te Measurements in Open-and Closed-Geometry Long-Scale-Length Laser Plasmas Via Isoelectronic X-Ray Spectralline Ratios, Lawrence Livermore National Laboratory, Livermore, CA, UCRL-JC-116180 (1995); Rev. Sci. Inst. 66(1), 749-751 (1995).

Shore, B. W., Feit, M. D., Perry, M. D., Boyd, R. D., Britten, J. A., and Li, L., Diffractive Coherence in Multilayer Dielectric Gratings, Lawrence Livermore National Laboratory, Livermore, CA, UCRL-JC-120189 (1995). Prepared for 7 th Rochester Conf on Coherence $\&$ Quantum Optics, Rochester, NY, Jun 7-10, 1995. 
Shore, B. W., Feit, M. D., Perry, M. D., Boyd, R. D., Britten, J. A., and Li, L., Electric Field Enhancement in Metallic and Multilayer Dielectric Gratings, Lawrence Livermore National Laboratory, Livermore, CA, UCRL-JC-121128 (1995). Prepared for 1st Annual Int'l Conf on Solid-State Lasers for Application to Inertial Confinement Fusion, Monterey, CA, May 30-Jun 2, 1995.

Shore, B. W., Stuart, B. C., Feit, M. D., Rubenchik, A. M., and Perry, M. D., Laser Induced Damage in Multilayer Dielectric Gratings Due to Ultrashort Laser Pulses, Lawrence Livermore National Laboratory, Livermore, CA, UCRL-JC-120187 (1995). Prepared for 1st Annual Int'l Conf on Solid-State Lasers for Application to Inertial Confinement Fusion, Monterey, CA, May 30-Jun 2, 1995.

Skidmore, J., High-Power CW $690 \mathrm{~nm}$ Al Gain P LaserDiode Arrays, Lawrence Livermore National Laboratory, Livermore, CA, UCRL-JC-117679 (1995); Appl. Phys. Lett. 66(3), 1163-1165 (1995).

Skidmore, J., High-Power CW Operation of Al Gain P Laser-Diode Arrays, Lawrence Livermore National Laboratory, Livermore, CA, UCRL-JC-115945 (1995); IEEE/J. Quant. Elect. 66(2), 133-135 (1995).

Strobel, G. L., and Haan, S. W., Source for Indirect Drive NIF Preheat Studies, Lawrence Livermore National Laboratory, Livermore, CA, UCRL-JC-120919 ABS (1995). Prepared for Symp on Fusion Engineering, Champaign, IL, Sept 30-Oct 5, 1995.

Stuart, B., Broadband Amplification in Ti:Sapphire Beyond $1 \mathrm{fm}$, Lawrence Livermore National Laboratory, Livermore, CA, UCRL-JC-115176 (1995); IEEE J. Quant. Elect. 31(3), 528-538 (1995).

Stuart, B., Laser Induced Damage in Dielectrics with Nanosecomd to Subpicosecond Pulses, Lawrence Livermore National Laboratory, Livermore, CA, UCRL-JC-118175 (1995); Phys. Rev. Lett. 74(3), 2248-2251 (1995).

Suter, L. J., Pollaine, S. M., and Powers, L. V., Power Balance and Radiation Production in Laser Heated Hohlraums, Lawrence Livermore National Laboratory, Livermore, CA, UCRL-JC-120790 ABS (1995). Prepared for 25th Annual Anomalous Absorption Conf, Aspen, CO, May 27-Jun 1, 1995.

\section{T}

Tabak, M., What Is the Role of Tritium Poor Fuels in ICF? Lawrence Livermore National Laboratory, Livermore, CA, UCRL-JC-118168 Rev. 1 (1995). Submitted to Nuclear Fusion.

Tietbohl, G., Dorsey, G., Frank, N., Marion, J., Murray, J., Murray, J., Riddle, B., Roberts, D., Sutton, S., and Taylor, J., Incident Analysis Report; Failure of a Spatial Filter Lens on the Beamlet Laser at LLNL on 4/3/95, Lawrence Livermore National Laboratory, Livermore, CA, UCRL-ID-120912 (1995).

Tobin, M., Anderson, A., Latkowski, J., Singh, M., Marshall, C., and Bernat, T., Physics Issues Related to the Confinement of ICF Experiments in the U.S. National Ignition Facility, Lawrence Livermore National Laboratory, Livermore, CA, UCRL-JC-119378 (1995). Prepared for 12th Int'l Conf on Laser Interaction and Related Plasma Phenomena, Osaka, Japan, Apr 24-28, 1995.

\section{V}

Van Wonterghem, B. M., Caird, J. A., Barker, C. E., Campbell, J. H., Murray, J. R., and Speck, D. R., Performance of the Beamlet Laser, a Testbed for the National Ignition Facility, Lawrence Livermore National Laboratory, Livermore, CA, UCRL-JC-121272 (1995). Prepared for Laser Optics '95, St Petersburg, Russia, Jun 27-Jul 1, 1995.

Van Wonterghem, B. M., Caird, J. A., Barker, C. S., Campbell, J. H., Murray, J. R., and Speck, D. R., Recent Results of the National Ignition Facility Beamlet Demonstration Project, Lawrence Livermore National Laboratory, Livermore, CA, UCRL-JC-120917 ABS (1995). Prepared for 1st Annual Int'l Conf on Solid-State Lasers for Application to Inertial Confinement Fusion, Monterey, CA, May 30-Jun 2, 1995.

Vogel, D., High-Pressure Positron-Sensitive Proportional Counter, Lawrence Livermore National Laboratory, Livermore, CA, UCRL-JC-117449 (1995); Rev. Sci. Inst. 66(1), 776-778 (1995).

Vu, B. T., Time-Resolved Probing of Femtosecond-LaserProduced Plasmas in Transparent Solids by Electron Thermal Transport, Lawrence Livermore National Laboratory, Livermore, CA, UCRL-JC-117456 (1995); Phys. Plasmas 2(2), 476-485 (1995). 


\section{W}

Warhus, J., Mast, J., and Nelson, S., Imaging Radar for Bridge Deck Inspection, Lawrence Livermore National Laboratory, Livermore, CA, UCRL-JC-120901 (1995). Prepared for Nondestructive Evaluation of Aging Bridges and Highways, Oakland, CA, Jun 6-8, 1995.

Warner, B. E., Boley, C. D., Chang, J. J., Dragon, E. P., Havstad, M. A., and McLean II, W., Industrial Applications of High Power Copper Vapor Lasers, Lawrence Livermore National Laboratory, Livermore, CA, UCRL-JC-120702 ABS (1995). Prepared for NATO Advanced Research Workshop on Pulsed Metal Vapour Lasers, St Andrews, Scotland, Aug 7-10, 1995.

Warner, B., Boley, C., Chang, J., Dragon, E., Havstad, M., Martinez, M., and McLean II, W., Ablative Material Removal Utilizing the Copper Vapor Laser, Lawrence Livermore National Laboratory, Livermore, CA, UCRL-JC-121130 ABS (1995). Prepared for Lasers and Electro Optics Society '95 8th Annual Mtg, San Francisco, CA, Oct 30-Nov 2, 1995.

Weber, S. V., Dalhed, S., Eimerl, D., Key, M. H., and Verdon, C. P., NIF Direct Drive Capsule Design Studies, Lawrence Livermore National Laboratory, Livermore, CA, UCRL-JC-120759 ABS (1995). Prepared for 25th Annual Anomalous Absorption Conf, Aspen, CO, May 27-Jun 1, 1995.

Wegner, P. J., Feit, M. D., Fleck, A. J., and Eimerl, D., Measurement of the Bespalov-Talanov Gain Spectrum in a Dispersive Medium with Large $\mathrm{N}_{2}$, Lawrence Livermore National Laboratory, Livermore, CA, UCRL-JC-121196 (1995). Prepared for 1st Annual Int'l Conf on Solid-State Lasers for Application to Inertial Confinement Fusion, Monterey, CA, May 30-Jun 2, 1995.

Wegner, P., and Feit, M. D., High-Power Narrow-Band Pulses with Wavelength Tunable about $1.053 \mu \mathrm{m}$ from a Synchronously-Pumped Optical Parametric Oscillator, Lawrence Livermore National Laboratory, Livermore, CA, UCRL-JC-120908 (1995). Submitted to Appl. Opt.

Wilks, S. C., and Kruer, W. L., Nonlinear Aspects of SBS in Multi-Species Plasmas, Lawrence Livermore National Laboratory, Livermore, CA, UCRL-JC-120882 ABS (1995). Prepared for 25th Annual Anomalous Absorption Conf, Aspen, CO, May 27-Jun 1, 1995.
Wilks, S. C., Critical Issues Regarding Ultra-Intense, Short-Pulse Laser-Plasma Interactions in the Fast Ignitor, Lawrence Livermore National Laboratory, Livermore, CA, UCRL-JC-120885 ABS (1995). Prepared for 1995 Canadian Association of Physicists' 50 th Anniversary Congress CAM-95, Quebec City, Quebec, Canada, Jun 11-16, 1995.

Wilks, S. C., Kruer, W. L., Hammel, B., Hammer, J., Tabak, M., and Young, P. E., Fast Ignitor Plasma Physics Issues, Lawrence Livermore National Laboratory, Livermore, CA, UCRL-JC-120884 ABS (1995). Prepared for 25th Annual Anomalous Absorption Conf, Aspen, CO, May 27-Jun 1, 1995.

Wilks, S. C., Kruer, W. L., Young, P. E., Hammer, J., and Tabak, M., Ultra-Intense, Short Pulse Laser-Plasma Interactions with Applications to the Fast Ignitor, Lawrence Livermore National Laboratory, Livermore, CA, UCRL-JC-121326 (1995). Prepared for 12th Int'l Conf on Laser Interaction and Related Plasma Phenomena, Osaka, Japan, Apr 24-28, 1995.

Wilks, S., Stimulated Raman Backscatter in Ultraintense, Short Pulse Laser-Plasma Interactions, Lawrence Livermore National Laboratory, Livermore, CA, UCRL-JC-118111 (1995); Phys. Plasmas 2(1), 274-279 (1995).

Williams, E. A., and Hinkel, D. E., Beam Steering by Filamentation in Flowing Plasmas, Lawrence Livermore National Laboratory, Livermore, CA, UCRL-JC-120770 ABS (1995). Prepared for 25th Annual Anomalous Absorption Conf, Aspen, CO, May 27-Jun 1, 1995.

Williams, E. A., Nonlinear Frequency Shifts and Harmonic Generation in Finite Amplitude Ion Acoustic Waves, Lawrence Livermore National Laboratory, Livermore, CA, UCRL-JC-120769 ABS (1995). Prepared for 25th Annual Anomalous Absorption Conf, Aspen, CO, May 27Jun 1, 1995.

Williams, E., The Frequency and Damping of Ion Acoustic Waves in Hydrocarbon ( $\mathrm{CH}$ ) and Two-Ion-Species Plasmas, Lawrence Livermore National Laboratory, Livermore, CA, UCRL-JC-118343 (1995); Phys. Plasmas 2(1), 129-138 (1995). 
Wolfe, C. R., and Lawson, J. K., The Measurement and Analysis of Wavefront Structure from Large Aperture ICF Optics, Lawrence Livermore National Laboratory, Livermore, CA, UCRL-JC-121148 (1995). Prepared for 1st Annual Int'l Conf on Solid-State Lasers for Application to Inertial Confinement Fusion, Monterey, CA, May 30-Jun 2, 1995.

Wolfe, C. R., Lawson, J. K., Aikens, D. M., and English, R. E., A Database of Wavefront Measurements for: Laser System Modeling, Optical Component Development and Fabrication Process Qualification, Lawrence Livermore National Laboratory, Livermore, CA, UCRL-JC-120767 (1995). Prepared for Interferometry VII Applications, Tucson, AZ, Jul 14, 1995.

Wolfe, C. R., Lawson, J. K., Manes, K. R., Aikens, D. M., and English, R. E., Specification of Optical Components Using the Power Spectral Density Function, Lawrence Livermore National Laboratory, Livermore, CA, UCRL-JC-120920 ABS (1995). Prepared for Society of Photo-Optical Instrumentation Engineers' 40th Annual Mtg, San Diego, CA, Jul 9-14, 1995.

Woodworth, J. G., Kreisler, M. N., and Kerman, A. K., A Free Wave Accelerator, Lawrence Livermore National Laboratory, Livermore, CA, UCRL-JC-120712 (1995). Prepared for Tamura Symp, Austin, TX, Nov 14-16, 1995.
Wu, C. H., Zory, P. S., and Emanuel, M. A., Characterization of Thin p-Clad In GaAs Single Quantum Wall Lasers, Lawrence Livermore National Laboratory, Livermore, CA, UCRL-JC-120237 (1995). Submitted to Photonics Technology Letters.

\section{$\mathbf{Y}$}

Yang, T.-Y. B., Kruer, W. L., Langdon, A. B., and More, R. M., Collisionless Absorption of Light Waves Obliquely Incident on Overdense Plasmas with Sharp Density Gradients, Lawrence Livermore National Laboratory, Livermore, CA, UCRL-JC-120772 ABS (1995). Prepared for 25th Annual Anomalous Absorption Conf, Aspen, CO, May 27-Jun 1, 1995.

\section{$\mathbf{Z}$}

Zaitseva, N. P., De Yoreo, J. J., Montgomery, K. E., Vital, R. L., De Haven, M. R., and Richardson, M. J., The Effect of Impurities at Rapid Growth of KDP Crystals, Lawrence Livermore National Laboratory, Livermore, CA, UCRL-JC-120242 ABS (1995). Prepared for 14th Conf on Crystal Growth and Epitaxy, Fallen Leaf Lake, CA, Jun 4-7, 1995.

Zakharenkov, Y. A., Hay, R. G., Darrow, C. B., Fews, A. P., Hammel, B. A., Nguyen, H., and Perry, M. D., Energetic Ions, Produced in Short-Pulse-Laser Interaction with Solid Targets, Lawrence Livermore National Laboratory, Livermore, CA, UCRL-JC-120762 ABS (1995). Prepared for 25th Annual Anomalous Absorption Conf, Aspen, CO; May 27-Jun 1, 1995. 